\title{
Environmental Effects of the Beaufort Lens on Underwater Acoustic Communications during Arctic Operations
}

by

Daniel Wilson Goodwin

B.S., Rose-Hulman Institute of Technology, 2008

Submitted to the Department of Mechanical Engineering and the Joint Program in Applied Ocean Science \& Engineering

in Partial Fulfillment of the Requirements for the Degree of

Master of Science in Mechanical Engineering

at the

MASSACHUSETTS INSTITUTE OF TECHNOLOGY

and the

\section{WOODS HOLE OCEANOGRAPHIC INSTITUTION}

September 2021

(C) 2021 Daniel W Goodwin. All rights reserved.

The author hereby grants to MIT and WHOI permission to reproduce and to distribute publicly paper and electronic copies of this thesis document in whole or in part in any medium now known or hereafter created.

Author

Department of Mechanical Engineering, MIT Joint Program in Applied Ocean Science \& Engineering, WHOI

August 06, 2021

Certified by

Henrik Schmidt

Professor of Mechanical and Ocean Engineering, MIT Thesis Supervisor

Accepted by

Nicolas Hadjiconstantinou

Professor of Mechanical Engineering, MIT Chairman, Committee for Graduate Students

Accepted by

David Ralston Associate Scientist with Tenure, Applied Ocean Physics \& Engineering, WHOI Chairman, Joint Committee for Applied Ocean Science \& Engineering 


\title{
Environmental Effects of the Beaufort Lens on Underwater Acoustic Communications during Arctic Operations
}

by

\author{
Daniel Wilson Goodwin
}

\begin{abstract}
Submitted to the Department of Mechanical Engineering and the Joint Program in Applied Ocean Science \& Engineering on August 06, 2021 in Partial Fulfillment of the Requirements for the Degree of Master of Science in Mechanical Engineering
\end{abstract}

\begin{abstract}
Operations in the Arctic Ocean are increasingly important due to the changing environment and the resulting global implications. These changes range from the availability of new global trade routes, accessibility of newly available resources in the area, and national security interests of the United States in the region. It's necessary to build a greater understanding of the undersea environment and how it's changing since these environmental changes have a direct impact on adjusting future operations in the region and looming global changes as less Arctic ice is present. The recent presence of the Beaufort Lens is changing the acoustic propagation paths throughout the Arctic region. Here a network of buoys were employed to communicate with an Autonomous Undersea Vehicle (AUV) while it operated under the ice throughout the Beaufort Lens with the goal of achieving near GPS quality navigation. The acoustic communications paths were compared using a vertical array throughout the Beaufort Lens. This beam forming was compared to the prediction from BELLHOP. As well, since acoustic communications are affected by multi-path, attenuation and interference from other sources it was interesting to note that bottom bounce was sometimes a reliable acoustic path.
\end{abstract}

Thesis Supervisor: Henrik Schmidt

Title: Professor of Mechanical and Ocean Engineering, MIT 


\section{Acknowledgments}

First and foremost I would like to extend my sincere gratitude to the Navy's Civilian Institution program, along with the MIT-WHOI Joint Program for making this opportunity possible for me and the Naval Officers who preceded me. I'd like to thank the U.S. Navy Submarine Force for supporting me through this next phase of my career, and enabling me to work alongside the nation's subject matter experts in ocean acoustics. A special thanks to the officers and crews I served alongside while assigned to USS Greeneville (SSN 772) and USS Connecticut (SSN 22). It was their grit, relentless determination, and motivation to succeed that inspired me to continue working hard in my career with the Navy. Those experiences guided me personally and professionally, leading to this unique opportunity at MIT-WHOI to continue my professional growth as an ocean acoustician.

Next I would like to thank professor Henrik Schmidt. His leadership and constant professionalism were wonderful to witness. As well, professor Schmidt was an incredible mentor to me throughout the preparation process for ICEX 2020, and throughout my learning experience. He always found a way to make time, and fostered learning in such an open way that never stymied my own growth or curiosity. Professor Schmidt was a phenomenal role model and someone I aspire to emulate in my own life moving forward. I would also like to thank those apart of the Lab for Autonomous Marine Sensing Systems (LAMSS), professor Mike Benjamin, Dr. Eeshan Bhatt, and Dr. Rui Chen. Most notably, I'd like to mention soon to be Dr. Oscar Viquez for his immense patience and constant help throughout my time in the program.

I would not have been able to accomplish this work without the coordination I received from Lincoln Laboratories Group 37, Advanced Undersea Systems and Technology. The Group Leader, Dr. Brian Lewis, was instrumental in procuring my access to Lincoln Labs to evaluate the DURIP towed array data from ICEX 2020, and ensure professor Henrik Schmidt was able to meet with me for my thesis meetings. As a fellow submariner, and professional mentor to me, Ron Ross was always available to talk and bounce ideas off of. Dr. Jon Collis was the arctic and acoustics subject mater expect. He helped me with every element of interactions with Lincoln Lab, and processing the DURIP towed array data. His support throughout the process was critical to my ability to accomplish this work. Lastly, professor Arthur Baggeroer made his time available for thesis discussions, and his input was 
invaluable in providing context and perspective.

Last, yet never least, is my family. That includes my parents, Wilson and Deborah Goodwin, and my wife Keri's parents, Greg and Sally Ihde, who've played an incredible role shaping us as people, modeling healthy relationships for us to aspire to, and their willingness to provide help at a moments notice. Keri was there for me throughout the tragic loss of one of my closest friends in the Navy, and she rose to the occasion of a global pandemic as a Nurse Anesthetist to show up in the Intensive Care Units to supervise multiple patients on ventilators, suffering from SARS-CoV-2, better known as COVID-19. Her resilience in the face of the largest global disaster of our lifetimes was amazing, and continues to inspire me to this day. I wouldn't be able to do my work without her daily love and support. As well, my two children, Eliott and Luella, I love you both and getting to be a full-time dad during the peak of the pandemic while attempting to attend online ZOOM class from MIT was an experience I'll never forget. You two constantly re-ignite my internal flame of curiosity, and may yours continue to burn bright for a lifetime of learning ahead. 
THIS PAGE INTENTIONALLY LEFT BLANK 


\section{Contents}

1 Introduction $\quad 25$

1.1 Motivation . . . . . . . . . . . . . . . . . 25

1.1.1 Research Objectives . . . . . . . . . . . . . . . . 26

1.2 The Changing Arctic Environment . . . . . . . . . . . . 27

1.2 .1 Sound Speed Profile $(\mathrm{SSP}) \ldots \ldots \ldots \ldots$. . . . . . . . . . 27

1.2.2 The Beaufort Lens . . . . . . . . . . . . . . . . . . . . . 29

1.3 ICEX 2020 Experiment Design . . . . . . . . . . . . . . . . . 31

1.3.1 DURIP Towed Array . . . . . . . . . . . . . . . . . . . . 34

1.3.2 Harmonics and Subharmonics . . . . . . . . . . . . . . . . 36

1.3.3 Power Spectral Density throughout the Vertical Run . . . . . . . . . 37

2 Background in Fundamentals of Ocean Acoustics and Digital Signal Pro$\begin{array}{ll}\text { cessing with Beamforming } & 41\end{array}$

2.1 Ocean Acoustics and Sound Waves . . . . . . . . . . . . . . . . . . 42

2.1 .1 The Wave Equation . . . . . . . . . . . . . . . . . . . . 42

2.1.2 Snell's Law and Ray Tracing . . . . . . . . . . . . . . . . . . . . 43

2.1.3 Transmission Loss . . . . . . . . . . . . . . . . . . . . . . . 44

2.1 .4 Attenuation . . . . . . . . . . . . . . . . . . 45

2.1.5 Acoustic Reciprocity . . . . . . . . . . . . . . . . . . 46

2.1.6 BELLHOP . . . . . . . . . . . . . . . . . . . . . . . . . . 48

2.2 Digital Signal Processing . . . . . . . . . . . . . . . . . . . . . . . . . . . 49

$2.2 .1 \quad$ Aliasing . . . . . . . . . . . . . . . . . . . . . 49

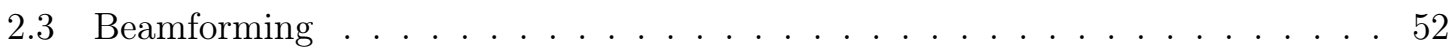

2.3.1 Beamforming Fundamentals for Linear Arrays . . . . . . . . . . . . 53 
2.3.2 Plotting Beam Patterns . . . . . . . . . . . . . . . 55

2.3 .3 Grating Lobes . . . . . . . . . . . . . . . . . . 56

2.3.4 Steering the Beams . . . . . . . . . . . . . . . . . 58

3 ICEX 2020 Experiment $\quad 61$

3.1 Ice Camp Seadragon . . . . . . . . . . . . . . . . . . . . . . 6 61

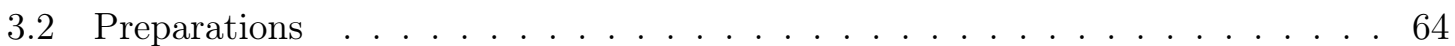

3.3 Minor Complications . . . . . . . . . . . . . . . . 67

$3.3 .125 \mathrm{kHz}$ Modem physical damage . . . . . . . . . . . . . 67

3.3 .2 Main Electronics Housing $(\mathrm{MEH}) \ldots \ldots$. . . . . . . . . . . . 69

3.3.3 LAMSS Hard Drive Failure . . . . . . . . . . . . . . . 70

3.4 Major Complications . . . . . . . . . . . . . . . . . . 71

3.4 .1 Vehicle Fault . . . . . . . . . . . . . . . . . . . 71

3.4 .2 Immediate Position Mark . . . . . . . . . . . . . . . . 71

3.4.3 Vehicle Verification and Daisy Chain Recovery Method . . . . . . . . . 73

3.4 .4 Final Recovery Planning . . . . . . . . . . . . . . . 75

3.4.5 Vehicle Recovery Procedure . . . . . . . . . . . . . . . . . 75

4 ICEX DURIP Array Analysis $\quad 79$

4.1 DURIP Towed Array Beamforming Specifics . . . . . . . . . . . . . . . 79

4.1.1 DURIP Towed Array Spatial Aliasing or Grating . . . . . . . . . . . 79

4.1.2 DURIP Towed Array Full Beamforming with all Elements . . . . . . . 82

4.1 .3 Hanning Window Weighting . . . . . . . . . . . . . . . . . . . . 84

4.2 ICEX DURIP Data Analysis . . . . . . . . . . . . . . . . . . 92

4.2.1 BELLHOP Projections . . . . . . . . . . . . . . . . 92

4.2.2 DURIP Array Vertical Run Beamforming . . . . . . . . . . . . . . 97

4.3 Arrival Angle Analysis . . . . . . . . . . . . . . . . . . . . . 110

4.3.1 H1 Analysis with Source Depth, $z_{s},=90 \mathrm{~m} \ldots \ldots 111$

4.3.2 H2 Analysis with Source Depth, $z_{s},=30 \mathrm{~m} \ldots \ldots . . \ldots 115$

5 Communications Signal Analysis $\quad 117$

5.1 One Way Travel Time $(\mathrm{OWTT})$. . . . . . . . . . . . . . . . 117

5.1 .1 OWTT Potential Transmissions . . . . . . . . . . . . . 118 
5.2 Arrival Path . . . . . . . . . . . . . . . . . . . . . . . . . . 119

5.2.1 Buoy Transmission Window Analysis . . . . . . . . . . . . . . . . . 121

5.2 .2 Macrura Transmission Window Analysis . . . . . . . . . . . . . . . . . 122

5.3 Wave-Front Range Analysis from OWTT . . . . . . . . . . . . . 123

5.3 .1 Total Water Depth . . . . . . . . . . . . . . . . . . . 123

5.3.2 OWTT BB vs Direct Path or Surface Reflected Ranges . . . . . . . . 125

5.3.3 BB Arrivals from H1 to H3 During Simulated Run . . . . . . . . . . . 128

6 Conclusions and Future Work

6.1 Review and Conclusion . . . . . . . . . . . . . . . . . 133

6.2 Future Work Recommendations . . . . . . . . . . . . . . . . . 135

$\begin{array}{ll}\text { A Vehicle Recovery Procedure } & 137\end{array}$

A.1 Recovery Images . . . . . . . . . . . . . . . . . . . . . 137

B DURIP Towed Array Beam Patterns 149

B.1 23 Element Polar Beam Patterns . . . . . . . . . . . . . . . . . . . . . . 149

B.2 23 Element u-space Beam Patterns . . . . . . . . . . . . . . . . 151

B.3 DURIP Towed Array Spatial Aliasing or Grating Reduction . . . . . . . . . 155

B.3.1 Transmission $\# 1473, z_{s}=30 \mathrm{~m}$, DURIP towed array at $\sim 120 \mathrm{~m} \ldots 156$

B.3.2 Macrura Transmission Grating Example . . . . . . . . . . . . . . . 163

B.4 Vertical Run Additional Transmissions . . . . . . . . . . . . . . . . . 170

$\begin{array}{lr}\text { Bibliography } & 183\end{array}$ 
THIS PAGE INTENTIONALLY LEFT BLANK 


\section{List of Figures}

1-1 A Generic Sound Speed Profile. [3] . . . . . . . . . . . . . . . . . 28

1-2 Arctic SSP with the propagation for a source depth of $100 \mathrm{~m}$. The ray diagram shows the sound doesn't interact with the bottom, but instead scattering occurs primarily from surface interactions. [3] . . . . . . . . . . . . . . . . 29

1-3 Typical deep water SSP with deep sound channel propagation. The source is at the depth of the deep sound channel axis [3]. . . . . . . . . . . . . . 30

1-4 Historically monotonically increasing SSP as compared to the SSP present in the Arctic clearly showing the presence of the Beaufort Lens. . . . . . . . . . 31

1-5 Variability of the SSP throughout a single day of AUV operations on the ice. These SSP are the raw XCTD data. . . . . . . . . . . . . . . . . . 32

1-6 ICEX 2020 Experiment Layout. The hydrohole was located topside, where there was a large hole in the ice for the AUV to go in and out of the water. Each buoy included two shallow $(30 \mathrm{~m})$ and two deep $(90 \mathrm{~m})$ receivers, but only a single transmitter that was shallow or deep. [7] . . . . . . . . . . 33

1-7 Physical design of DURIP towed array. . . . . . . . . . . . . . . . 34

1-8 DURIP towed array internal element layout. . . . . . . . . . . . . . . 35

1-9 Block Diagram for DURIP towed array and connections to Macrura. . . . . . 35

1-10 Spectrogram of a WHOI micro-modem transmission as recorded by element 16 from the DURIP towed array. . . . . . . . . . . . . 36

1-11 Vertical Run depth profile for Macrura. The vehicle was not moving during this time-period, and the transmissions from $\mathrm{H} 1$ at $90 \mathrm{~m}$ and $\mathrm{H} 2$ at $30 \mathrm{~m}$ are

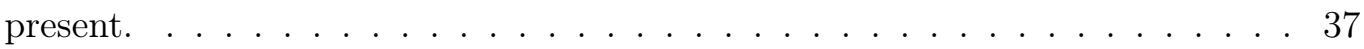

1-12 Top down view of Hydrohole and buoy locations with ranges . . . . . . . . . 38 
1-13 Power Spectral Density (PSD) for all elements on the towed array averaged together over multiple buoy communication transmissions from the WHOI micro-modems. . . . . . . . . . . . . . . . . . . . 39

1-14 WHOI micro-modem transducer source level. This is the transducer that was used on the buoys (H1, H2, H3, \& H4) during ICEX for transmitting. During ICEX the transducer was set to the blue line, $\mathrm{VH}=24$ Vrms. . . . . . . . . . 40

2-1 Longitudinal sound wave. How sound propagates. [15] . . . . . . . . . . . . 41

2-2 Ray paths, combined with Snell's Law enable ray tracing. This provides an approximation for how sound waves propagate through a medium and the trajectories the sound waves follow. This also means that the wave front bends. $[3] \ldots \ldots \ldots \ldots \ldots \ldots \ldots$

2-3 Regions of different dominant processes of attenuation of sound in sea water.

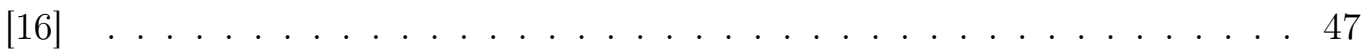

2-4 Acoustic reciprocity between two submarines [19] . . . . . . . . . . . . . . 48

2-5 BELLHOP example for the 1430UTC XCTD with $z_{s}=90 \mathrm{~m}$. Rays must be

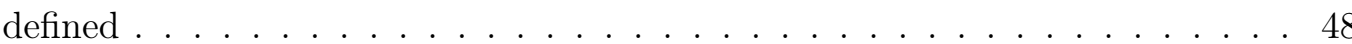

2-6 Aliasing for $x_{1}=\cos (0.4 \pi t)$. Although $x_{2}$ and $x_{3}$ are different functions, when $T_{s}=1$ second, the samples align with all three functions. As a result, they are said to be aliased. When looking at the frequency domain of the samples, it's clear that $x_{1}$ is effectively repeating itself every $\pm 2 \pi \mathrm{n}$ where $\mathrm{n}=$ $0,1,2, \ldots$ In this context, it's impossible to distinguish $x_{1}, x_{2}$, and $x_{3}$ from one another without adjusting $T_{s} \ldots \ldots \ldots \ldots \ldots$. . . . . . . . 50

2-7 During ICEX $f_{s}=12 \mathrm{kHz}$ for the DURIP towed array. The $10 \mathrm{kHz}$ transmissions from the WHOI micro-modem are under-sampled and alias down to 2

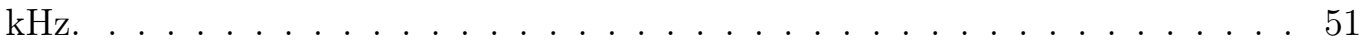

2-8 $f_{s}=12 \mathrm{kHz} . \mathrm{A} 10 \mathrm{kHz}$ and $2 \mathrm{kHz}$ signal are indistinguishable. . . . . . . 52

2-9 Coordinate system and linear array conventional element locations. [14] . . . 53

2-10 Polar plot and u-space plot of beam pattern for a 23 element linear array with

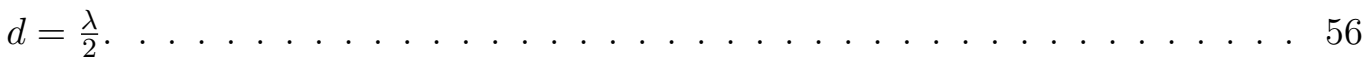

2-11 u-space visible region when $d=\lambda$ vice $d=\frac{\lambda}{2} \ldots \ldots \ldots \ldots$. . . . . 57

2-12 Polar plot with $d=\lambda$. Same as Figure 2-11b. . . . . . . . . . . . . 58 
2-13 Polar plot and u-space plot of beam patterns when array is steered to different angles. . . . . . . . . . . . . . . . . . . . . . . . . 59

3-1 MIT-WHOI ICEX team inside the operations tent. Hydrohole is beneath the wooden planks on the floor. From left: Dan McDonald (Bluefin), Bradli Howard, Dennis Giaya (WHOI), Josiah Delange (Bluefin, kneeling), Oscar Viquez, Dr. Toby Schneider (GobySoft), Dan Goodwin, Dr. Henrik Schmidt, and Dr. Rui Chen. . . . . . . . . . . . . . . . . . . . . 62

3-2 Aerial photograph while circling Ice Camp Seadragon to line-up with the runway. . . . . . . . . . . . . . . . . . . 63

3-3 Topside operations tent and the hydrohole. . . . . . . . . . . . . . . . . 64

3-4 Two TU-142 Russian reconnaissance aircraft overflying Ice Camp Seadragon while flanked by F-22s and FA-18s. . . . . . . . . . . . . . . . . . . 64

3-5 $25 \mathrm{kHz}$ modem incurred damage following basic operations testing, and the mount was destroyed. The transducer was re-positioned for further operations taking advantage of wood chips that were available at the ice camp. . . . . . . 68

3-6 Troubleshooting and replacing a card on the MEH. . . . . . . . . . . . . . 69

3-7 LAMSS hard drive failure and reformatting a new disk. . . . . . . . . . . 70

3-8 Final fix that was used to go mark the vehicle location. This was a 5-node fix since the portable hydrophone (ie. Towfish) was lowered into the hydrohole to maximize accuracy. . . . . . . . . . . . . . . . . . . . . 72

3-9 Image used to brief team on the Daisy Chain recovery plan and how the second line and clip would be re-used. . . . . . . . . . . . . . . . 73

4-1 Polar plot showing grating lobes of DURIP nested array (23-Elements) beamformed at $5 \mathrm{kHz} \ldots \ldots \ldots \ldots \ldots$. . . . . . . . . . . . . . . . . . . .

4-2 WHOI micro-modem transmission \#1893 from H1 $\left(z_{s}=90 \mathrm{~m}\right)$ captured on the DURIP towed array at a depth of roughly $159 \mathrm{~m}$. Here the direct path arrival, $+4^{\circ}$, is stronger and arrives slightly earlier compared to the surface reflected arrival which comes in at a steeper $+9.3^{\circ}$. The small time delay is due to the slightly longer path for the wave to travel upward into the ice, reflect, and arrive at the array vs. the direct path transmission. . . . . . . . . 81 
4-3 DURIP towed array beamforming comparison. The WHOI micro-modem transmission is not visible in 4 -3a. In $4-3 \mathrm{~b}$ the transmission is visible since this frequency spectrum includes the portion that captures the WHOI micromodem subharmonic at $5 \mathrm{kHz}$ and additional energy between $5-6 \mathrm{kHz}$. . . . . 82

4-4 Layout of DURIP towed array highlighting the 23 element nested array in the center with red triangles. . . . . . . . . . . . . . . . . . . 83

4-5 Uniform 23 element nested array vs. Full 32 element array beam pattern. . . 84

4-6 Nested array (23 elements) vs Full DURIP array (32 elements) beam patterns with uniform weights. . . . . . . . . . . . . . . 85

4-7 Plot of different normalization constants, and their impact on the beam pattern. 86

4-8 Left polar plot shows how Hanning weights affect the beam pattern of the 23 element nested array. The right plot shows how the Hanning weights affect the beam pattern of the full 32 element DURIP array. . . . . . . . . . . . 87

4-9 Polar plot of 23 element nested array with uniform weights compared to the full 32 element DURIP array with Hanning weights. . . . . . . . . . . . . 88

4-10 u-space comparison of 23 element nested array with uniform weights and the full 32 element DURIP array with Hanning weights. . . . . . . . . . . . . . . 89

4-11 u-space comparison of full DURIP array with uniform weights vs Hanning weights. See right polar plot Figure $4-8 \ldots \ldots$. . . . . . . . . . . . . . 89

4-12 The Hanning weights on the left are swathy compared to the uniform weights, although the uniform weights result in some array overdriving showing a horizontal line across all $\mathrm{D} /$ Es. . . . . . . . . . . . . . . . . . . . . . 90

4-13 The Hanning weights show a micro-modem transmission that was washed out by the uniform weights. Although the uniform weighting main lobe is narrower, the additional background noise results in a washed out signal due to the stronger side and back lobes. . . . . . . . . . . . . . . . 90

4-14 Vertical Run highlighting the transmission that were missed by Macrura. . . . 92

4-15 Ray traces from 1430UTC and 2100UTC with $z_{s}=90 \mathrm{~m} \ldots \ldots . . . . .99$

4-16 Ray traces from 1430UTC and 2100UTC with $z_{s}=30 \mathrm{~m} \ldots \ldots$. . . . 96

4-17 Vertical Run highlighting the transmissions that were captured at each depth. 97 
4-18 Transmission \#273. $z_{s}=\mathrm{H} 190 \mathrm{~m}$. Array $\sim 38 \mathrm{~m}$. Only a single arrival is clearly visible at roughly $+11.2^{\circ}$. The swathy nature checks with the variability seen in the water column from Figures $4-32$ and 4-33 . . . . . . . . . 98

4-19 Transmission \#543. $z_{s}=\mathrm{H} 2 \quad 30 \mathrm{~m}$. Array $\sim 38 \mathrm{~m} . \ldots \ldots 9$

4-20 Transmission \#873. $z_{s}=\mathrm{H} 230 \mathrm{~m}$. Array $\sim 82 \mathrm{~m}$. Transmission was captured in the shadow zone. It's slight but the transmission appears swathy between $+4^{\circ}$ and $+8^{\circ}$. It's possible it could be two separate arrival paths, but there isn't a clear separation between the two arrival angles. There is also a possible arrival at $+13^{\circ}$, although this seems unlikely based on Figures 4-32 and 4-33. 100

4-21 Transmission \#963. $z_{s}=\mathrm{H} 230 \mathrm{~m}$. Array $\sim 82 \mathrm{~m}$. Transmission was captured in the shadow zone. It's slight but the transmission appears lightly between $+4^{\circ}$ and $+8^{\circ}$. It's possible it could be two separate arrival paths, but it's so weak it's hard to tell. . . . . . . . . . . . . . . . . . . . 101

4-22 Transmission \#1458. $z_{s}=\mathrm{H} 230 \mathrm{~m}$. Array $\sim 120 \mathrm{~m} . \ldots . \ldots . . \ldots 2$

4-23 Transmission \#1653. $z_{s}=\mathrm{H} 190 \mathrm{~m}$. Array $\sim 120 \mathrm{~m}$. A slight time delay is present between the $+6.9^{\circ}$ and $+11.3^{\circ}$ arrivals. Both arrivals are broad and diffuse or "swathy." . . . . . . . . . . . . . . . . . . . . . 103

4-24 Transmission \#1773. $z_{s}=\mathrm{H} 190 \mathrm{~m}$. Array $\sim 127 \mathrm{~m}$. A slight time delay is present between the $+4.9^{\circ}$ and $+10^{\circ}$ arrivals. Transmission occurred during the final vehicle depth excursion from 82 to $121 \mathrm{~m}$. The direct path arrival is sharp and crisp, easily discernible even with the other interfering background noise. . . . . . . . . . . . . . . . . . . . . 104

4-25 Transmission \#1803. $z_{s}=$ H1 $90 \mathrm{~m}$. Array 135 m. Transmission occurred during the final vehicle depth excursion from 82 to $121 \mathrm{~m}$. The direct path arrival is sharp and crisp, easily discernible. . . . . . . . . . . . . . . 105

4-26 Transmission \#1818. $z_{s}=\mathrm{H} 190 \mathrm{~m}$. Array $\sim 139 \mathrm{~m}$. A slight time delay is present between the $+4.4^{\circ}$ and $+9.5^{\circ}$ arrivals. Transmission occurred during the final vehicle depth excursion from 82 to $121 \mathrm{~m}$. The direct path arrival is sharp and crisp, easily discernible. . . . . . . . . . . . . . 106 
4-27 Transmission \#1833. $z_{s}=\mathrm{H} 190 \mathrm{~m}$. Array $\sim 145 \mathrm{~m}$. A slight time delay is present between the $+5^{\circ}$ and $+10.2^{\circ}$ arrivals. Transmission occurred during the final vehicle depth excursion from 82 to $121 \mathrm{~m}$. The direct path arrival is sharp and crisp, easily discernible even with the background noise in the upper D/Es. . . . . . . . . . . . . . . . . . . . 107

4-28 Transmission \#1923. $z_{s}=\mathrm{H} 190 \mathrm{~m}$. Array $159 \mathrm{~m}$. A slight time delay is present between the $+5.1^{\circ}$ and $+10.9^{\circ}$ arrivals. Although not as sharp as \#1833, it's quite cleaner than \#1653 with a similar amount of background noise. Would decode more consistently than \#1653. . . . . . . . . . . . . . 108

4-29 Transmission \#2463. $z_{s}=\mathrm{H} 230 \mathrm{~m}$. Array $\sim 159 \mathrm{~m}$. Only a single arrival path is visible at $+5.2^{\circ}$. Potentially due to the close arrival angle for both a direct path and surface reflected transmission seen in Figure 4-32 or the BELLHOP ray traces in Figures 4-16a, and 4-16b. Both arrivals appear to merge and are indistinguishable. . . . . . . . . . . . . . . . . . 109

4-30 Expected arrival angle from the XCTD from 1430 vs. the HYCOM approximation for the SSP for a $90 \mathrm{~m}$ source depth. DURIP towed array data was used to determine the arrival angle from all $90 \mathrm{~m}$ transmissions at that array depth. As well, the vehicle's statics for receptions from H1 $\left(z_{s}=90 \mathrm{~m}\right)$ are included . . . . . . . . . . . . . . . . . . . . . 111

4-31 Expected arrival angle from the XCTD from 2100 vs. the HYCOM approximation for the SSP for a $90 \mathrm{~m}$ source depth. DURIP towed array data was used to determine the arrival angle from all $90 \mathrm{~m}$ transmissions at that array depth. As well, the vehicle's statics for receptions from H1 $\left(z_{s}=90 \mathrm{~m}\right)$ are included. . . . . . . . . . . . . . . . . . . . . . . . . 112

4-32 Expected arrival angle from the XCTD from 1430 vs. the HYCOM approximation for the SSP for a $30 \mathrm{~m}$ source depth. DURIP towed array data was used to determine the arrival angle from all $30 \mathrm{~m}$ transmissions at that array depth. As well, the vehicle's statics for receptions from $\mathrm{H} 2\left(z_{s}=30 \mathrm{~m}\right)$ are included . . . . . . . . . . . . . . . . . . . . . . . 113 
4-33 Expected arrival angle from the XCTD from 2100 vs. the HYCOM approximation for the SSP for a $30 \mathrm{~m}$ source depth. DURIP towed array data was used to determine the arrival angle from all $30 \mathrm{~m}$ transmissions at that array depth. As well, the vehicle's statics for receptions from $\mathrm{H} 2\left(z_{s}=30 \mathrm{~m}\right)$ are included. . . . . . . . . . . . . . . . . . . . . . 114

5-1 Histogram of $\mathrm{H} 1$ arrivals throughout ICEX. . . . . . . . . . . . . . . 119

$5-2 \quad$ Histogram of $\mathrm{H} 2$ arrivals throughout ICEX. . . . . . . . . . . . 120

5-3 Histogram of $\mathrm{H} 3$ arrivals throughout ICEX. . . . . . . . . . . . 120

5-4 Histogram of $\mathrm{H} 4$ arrivals throughout ICEX. . . . . . . . . . . . . 121

5-5 $\quad$ Bathymetry at Ice Camp Seadragon [27] . . . . . . . . . . . . . . . . . . 122

5-6 Wave-Front of Macrura transmission based on OWTT during the Vertical Run. The direct or surface reflected OWTT was based off a reception while the vehicle was operating at $82 \mathrm{~m}$ since no direct or surface reflected OWTT occurred while the vehicle was at $121 \mathrm{~m}$ depth. . . . . . . . . . . . . . 124

5-7 Spread of TWD that makes sense for OWTT arrival at buoy H3. Note the TWD adjustments have no bearing on the direct path or surface reflected arrival path to $\mathrm{H} 3$ (blue curve). . . . . . . . . . . . . . . . . . . . . . 125

5-8 OWTT comparison for the same transmission received twice at buoy H3. . . 126

5-9 OWTT comparison for the same transmission received twice at buoy H3, but the BB arrival was "bad" according to "CACST" but was viable according to the carrier frequency of $10000 \mathrm{~Hz}$. These were both yellow in Figure 5-3. . 127

5-10 The BB transmission at H2 was not considered a "good" reception according to the "CACST" message. However, based on the $10000 \mathrm{~Hz}$ carrier frequency analysis, the message met the criteria for an acceptable message. This is one of the yellow potentially viable arrivals from Figure $5-2 . \quad \ldots . . .129$

5-11 OWTT comparison for transmissions received at H3 originating from $\mathrm{H} 1$ while H1 was a simulated vehicle on 03-10-2020. The shallow arrival was from 17:13:16, and the BB arrivals were from 19:17:46 to 19:24:46. Eleven BB arrivals occurred in that window that were all good according to "CACST." . 131 
A-1 Diagrams used to brief the recovery team on the plan. Helped convey the importance of the spacing between holes and positioning of the tripod during vehicle removal. . . . . . . . . . . . . . . . . . . . . . . 137

A-2 Returning to the ice camp following the snowstorm. The operations tent and the hydrohole were in need of serious repair. . . . . . . . . . . . . . . 138

A-3 After digging the first hole, without breaking through the ice, an ASL operator is inside the hole using a chainsaw to extend the upper corner. Since the two holes end to end were only $64 "$ long. This additional length at the top was intended for the nose of the vehicle to go into. It would reduce the angle that the vehicle was going to undergo during recovery. The ice anchor is present to prevent the ice chunk from falling into the hole where the operator is. . . . 139

A-4 This is the 36" auger that was used for drilling out the two holes. We decided on two based on the expertise from ASL. From their years of experience on the ice, it was clear that doing 2-holes with the first dry was achievable, but to attempt to do more holes (3 or 4 ) without breaking through the ice is extremely challenging. . . . . . . . . . . . . . . . . . . . . . . 140

A-5 Macrura being removed from the recovery hole. The two holes were separated by 28 ". This provided just over $22 "$ at the narrowest point for the vehicle to fit through. The vehicle is $21^{\prime \prime}$ in diameter. This distance was to maximize the length of the two holes while ensuring the vehicle would fit through the narrowest part. . . . . . . . . . . . . . . . . . . . . 14

A-6 The chain fall is connected to the FWD weight bearing mount on the vehicle. The nose handle and the side handle were not used to extract the vehicle. Those handles are not weight bearing mounts. . . . . . . . . . . . . . . . . . 142

A-7 This helps show just how tight it was for the vehicle to squeeze through the narrowest part of the two holes. As well, the slot cut in the front of the holes with the chainsaw was critical in reducing the angle of removal on the vehicle. The angle was roughly $50^{\circ} \ldots \ldots$. . . . . . . . . . . . . . . . . . . . . . . .

A-8 Macrura is fully out of the water and the DURIP towed array is being removed from the water and disconnected. . . . . . . . . . . . . . . . . . . . 144 
A-9 The vehicle fully removed with both chain falls being used. One connected to the FWD mount and one to the AFT mount. The 36" auger is in the background. The two spools of cable used to recover the vehicle are on the bottom left. The blue line was used to tie off the vehicle to topside through the duration of the snowstorm. . . . . . . . . . . . . . . 145

A-10 Macrura fully removed from the water awaiting transit back to ice camp. . . . 146

A-11 The vehicle was tied off to a snow sled and towed behind a snowmobile back to ice camp for disassembly and transit back to Prudhoe Bay, AK. . . . . . 147

B-1 Polar plots of 23 element nested array with $d=\frac{1-1.5 \lambda}{2} \ldots \ldots$. . . . . 149

B-2 Polar plots of 23 element nested array with $d=\frac{2-4.5 \lambda}{2} \ldots \ldots . \ldots 150$

B-3 Polar plots of 23 element nested array with $d=\frac{5-6 \lambda}{2} \ldots \ldots$. . . . . . . 151

B-4 23 Element nested array plotted in u-space with $d=\frac{\lambda}{2} \ldots \ldots$. . . . . 151

B-5 23 Element nested array in u-space with $d=\frac{1.5-2.5 \lambda}{2} \ldots \ldots . \ldots . \ldots . . \ldots 2$

B-6 23 Element nested array in u-space with $d=\frac{3-4 \lambda}{2} \ldots \ldots \ldots$. . . . . . 153

B-7 23 Element nested array in u-space with $d=\frac{4.5-6 \lambda}{2} \ldots \ldots$. . . . . . 154

B-8 DURIP Towed Array beamforming between $f=500-1000 H z$, the designed spectrum for the array. The WHOI micro-modem transmission isn't visible in this spectrum, which makes sense considering the PSD in Figure 1-13. All of the energy from the WHOI micro-modem transmissions occur between 5-6 kHz. . . . . . . . . . . . . . . . . . . . 156

B-9 DURIP Towed Array beamforming between $f=500-6000 H z$. Although portions of this are in the frequency spectrum where grating lobes are present, it's a dramatic change from Figure B- 8 , where the WHOI micro-modem transmission isn't even visible. . . . . . . . . . . . . . . . . . . 157

B-10 DURIP Towed Array beamforming between $f=1000-6000 H z$. Since $f \geq$ $1000 \mathrm{~Hz}$ this includes spatial aliasing, but the impacts are limited due to the large group of frequencies that beamforming is performed over. . . . . . . . 158

B-11 DURIP Towed Array beamforming between $f=2000-6000 H z$. The grating lobes are clearly present and resulting in spatial aliasing of the sound near $10^{\circ}$ between 2.8 to 3.6 seconds. However, the aliased signals are still fairly swathy and not sharply defined. . . . . . . . . . . . . . . . . . 159 
B-12 DURIP Towed Array beamforming between $f=3000-6000 H z$. The aliasing is clearly worse, but much of the low frequency content has been removed from the beamforming. Only higher frequency signals are present in this analysis. . 160

B-13 DURIP Towed Array beamforming between $f=4000-6000 H z$. The spatial aliasing is bad. . . . . . . . . . . . . . . . . . . 161

B-14 DURIP Towed Array beamforming between $f=4950-5050 H z$. The spatial aliasing in this case has an uncanny resemblance to the aliasing we would anticipate from Figure 4-1. This clearly shows how spatial aliasing occurs. The grating lobes effectively show sound at angles that it isn't actually present at. The transmission that occurs between 2.8 and 3.6 seconds is now spatially aliased, and it's unclear what angle the original signal was arriving from. The array noise that shows up at the beam is aliased to $\pm 50^{\circ}$ and $\pm 22^{\circ}$ which checks with the polar plot. . . . . . . . . . . . . . . . 162

B-15 DURIP Towed Array beamforming between $f=500-1000 H z$, the designed spectrum for the array. The vehicle transmission should be in FWD endfire of the array but isn't visible in this spectrum. . . . . . . . . . . . . 163

B-16 DURIP Towed Array beamforming between $f=500-6000 H z$. The vehicle transmission is visible in FWD endfire of the array. . . . . . . . . . . . . 164

B-17 DURIP Towed Array beamforming between $f=1000-6000 H z$. The vehicle transmission is visible in FWD endfire of the array. . . . . . . . . . . . 165

B-18 DURIP Towed Array beamforming between $f=2000-6000 H z$. The vehicle transmission is visible in FWD endfire of the array. Some minor aliasing is becoming apparent. . . . . . . . . . . . . . . . 166

B-19 DURIP Towed Array beamforming between $f=3000-6000 H z$. The vehicle transmission is visible in FWD endfire of the array. Aliasing is causing almost all the beams to be washed out. FWD endfire remains the strongest, and actual source of the sound. . . . . . . . . . . . . . . . . . . 167

B-20 DURIP Towed Array beamforming between $f=4000-6000 H z$. The vehicle transmission is visible in FWD endfire of the array. Clear aliasing is happening. FWD endfire is still the most dominant angle for the transmission that is washing out the rest of the beams. . . . . . . . . . . . . 168 
B-21 DURIP Towed Array beamforming between $f=4950-5050 H z$. The vehicle transmission is visible in FWD endfire of the array. Aliasing is overwhelming here. The noise at $0^{\circ}$ is aliasing to $\pm 22^{\circ}$ and $\pm 50^{\circ}$. The original transmission angle is indeterminate now as aliasing dominates. Since the original transmission is present in the back lobe (Recall Figure 4-6b and 4-11) while the beam is steered to $90^{\circ}$ relative, the transmission is showing up subtly in the $0^{\circ} \mathrm{D} / \mathrm{E}$ as well by amplifying the already present signal there. . . . . . . . . 169

B-22 Vertical Run highlighting the additional transmissions that were captured at each depth. . . . . . . . . . . . . . . . . . . . 170

B-23 Transmission \#78. $z_{s}=\mathrm{H} 1 \quad 90 \mathrm{~m}$. Array $\sim 38 \mathrm{~m} . \ldots \ldots \ldots$

B-24 Transmission \#378. $z_{s}=\mathrm{H} 190 \mathrm{~m}$. Array $\sim 38 \mathrm{~m} . \ldots . . . . . . . .172$

B-25 Transmission \#858. $z_{s}=\mathrm{H} 2 \quad 30 \mathrm{~m}$. Array $\sim 82 \mathrm{~m} . \ldots \ldots \ldots$

B-26 Transmission \#1428. $z_{s}=\mathrm{H} 230 \mathrm{~m}$. Array $\sim 120 \mathrm{~m} . \ldots . \ldots . . . \ldots 174$

B-27 Transmission \#1473. $z_{s}=\mathrm{H} 230 \mathrm{~m}$. Array $\sim 120 \mathrm{~m} . \ldots \ldots . \ldots \ldots$

B-28 Transmission \#1578. $z_{s}=\mathrm{H} 190 \mathrm{~m}$. Array $\sim 120 \mathrm{~m}$. . . . . . . . . . 176

B-29 Transmission \#1893. $z_{s}=\mathrm{H} 190 \mathrm{~m}$. Array $\sim 159 \mathrm{~m} . \ldots \ldots$. . . . . . 177

B-30 Transmission \#2178. $z_{s}=\mathrm{H} 190 \mathrm{~m}$. Array $\sim 159 \mathrm{~m} . \ldots . \ldots . . . . . .178$

B-31 Transmission \#2193. $z_{s}=\mathrm{H} 190 \mathrm{~m}$. Array $\sim 159 \mathrm{~m} . \ldots . . . . . . . .179$

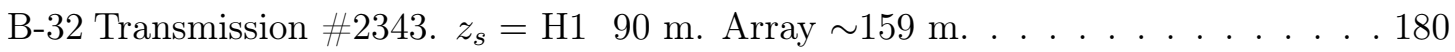

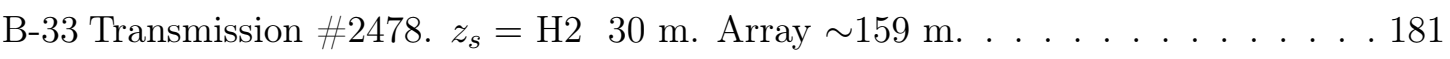

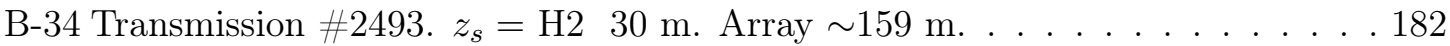


THIS PAGE INTENTIONALLY LEFT BLANK 


\section{List of Tables}

4.1 Vertical Run Depths . . . . . . . . . . . . . . . . . . 93

4.2 Transmissions from $\mathrm{H} 1, z_{s}=90 \mathrm{~m}$, and $\mathrm{H} 2, z_{s}=30 \mathrm{~m}$, received at buoys based on depth. . . . . . . . . . . . . . . . . . . . . . 94 
THIS PAGE INTENTIONALLY LEFT BLANK 


\section{Chapter 1}

\section{Introduction}

\subsection{Motivation}

In 2018 I was assigned to USS Connecticut (SSN 22), a fast-attack submarine stationed in Bremerton, WA. We were tasked with the unique responsibility to support a biennial exercise for the U.S. Submarine Force known as ICEX (Ice-Exercise). Not only does it only occur once every two years, but only two or three U.S. Submarines are involved with the event making the opportunity incredibly rare. I was genuinely excited to make the transit through the Bering Strait, separating Russia and Alaska, and ride through the gateway from the Pacific Ocean into the Arctic Ocean. A place so rarely experienced, beneath the ice, by fellow submariners.

Operating a submarine throughout the Arctic Ocean was life changing, and it inspired a great deal of interest for me in the region. Although I knew it was a critical part of the global ecosystem, I didn't fundamentally understand why that was the case. As well, the undersea domain was unlike anywhere else I'd experienced in the world. I'd previously deployed to the western pacific, and I worked as an instructor at Naval Submarine School preparing boats for European Command and Central Command deployments. Throughout those experiences I spent hours training boats about the different environments, their similarities, and their

differences. However, the Arctic Ocean was unlike any of the other environments I operated in or trained submariners to operate in.

During ICEX 2018, the Navy was supposed to shoot multiple practice torpedoes. This is a laborious process to execute under the ice because each torpedo is recovered after it's shot. During this exercise we were only able to shoot and recover a few torpedoes because 
of the difficulties encountered recovering the weapons. Typically it requires a dive team, digging holes through the ice to find the torpedo, and using a helicopter to lift the weapon out of the water through the hole. All subsequent shots are on hold until the torpedo is recovered. We were unable to shoot all the practice torpedoes because of the complications the environment created for finding the torpedoes at their final resting places underneath the ice.

This experience changed my perspective of the Arctic, and I became increasingly interested in the region and it's impact on the global ecosystem. It was increasingly apparent to me that mastery of the unique environment, and how it was changing, was necessary for the Navy to maintain its strength in the region.

\subsubsection{Research Objectives}

ICEX-2020 provided a unique opportunity to re-evaluate the Arctic environment and expand on the problems faced in 2018. Instead of searching for a torpedo, during ICEX-20 an Autonomous Undersea Vehicle (AUV) would operate in the environment and need to be recovered. However, the navigation system needed to be accurate enough that the vehicle would return to the hole we used to put it into the water. As well, no divers would be used or available to recover the AUV. This is a dramatic shift from the typical complexities faced attempting to recover torpedoes, and if the navigation system worked it would not only be an incredible success but could dramatically change future Arctic operations. What if a torpedo recovery only involved digging a hole next to the torpedo, a boat hook to drag the torpedo into the hole, and a helicopter to lift the vehicle out of the water. That's almost as easy as open ocean torpedo recovery except for digging a hole through the ice.

I specifically focused on the propagation paths the acoustic communications waves took and the affects the environment had on the reliable acoustic path. Acoustic communications are affected by multi-path, attenuation, and interference from other sources. This was a unique opportunity to compare the acoustic communications received at the vehicle with the DURIP towed array drooping from the AUV in a vertical configuration as it went through the Beaufort Lens. These data streams are typically independent, but using them together would help us build a better understanding of the modeling for the environment, its limitations, and what the reliable acoustic communications path actually looked like. 


\subsection{The Changing Arctic Environment}

Nothing describes the dramatic changes we're seeing in the Arctic as simply as the title for the Department of the Navy's Strategic Blueprint for the Arctic a Blue Arctic [1]. The implications of those three words are incredibly clear. The the Arctic ice-cover we're accustomed to is changing, and going to look different in the years to come. This starts with the major transition from substantial ice-cover to larger and larger portions without ice. The hidden ocean beneath the Arctic ice is increasingly exposed, and rarely used trade routes and resources that were previously inaccessible are becoming within reach.

\subsubsection{Sound Speed Profile (SSP)}

The Sound Speed Profile (SSP) is the tool most often used in the Submarine Force to describe the oceanic environment. Sometimes it is referred to as the Sound Velocity Profile (SVP). This is largely due to the fact that acoustic waves follow Snell's Law and the SSP provides the reference used to describe the boundary conditions each acoustic wave experiences as it propagates throughout the undersea domain.

The SSP is directly related to the density of water in the ocean. The density of water is related to the static pressure, salinity, and temperature of the water. These are the three key components that combine to describe the SSP. These three factors are often used to model the SSP by using the equation [2].

$$
c=1449.2+4.6 T-0.055 T^{2}+0.00029 T^{3}+(1.34-0.01 T)(S-35)+0.016 z
$$

In the equation, and throughout the paper, the variables for speed of sound (c) in $\mathrm{m} / \mathrm{s}$, temperature $(\mathrm{T})$ in ${ }^{\circ} \mathrm{C}$, salinity $(\mathrm{S})$ in parts per thousand $(\%)$, and depth $(\mathrm{z})$ in meters.

An example of a generic SSP and the descriptions of the ocean layers is shown in Figure 1-1. Each layer of the oceanic environment results in different acoustic propagation paths. However, this generic SSP also conveys how different the Polar region SSP is.

The differences in the Polar regions are largely due to the ice-sheet that covers the ocean surface. As a result, the mixed layer tends to have much less impact from surface conditions such as wind. The Arctic mixed layer is comprised of a largely freshwater layer with fairly consistent temperatures near freezing. This region of low salinity is due to the ice melting. Although the resulting mixed layer SSP is different in the arctic, it's due to the ice conditions 


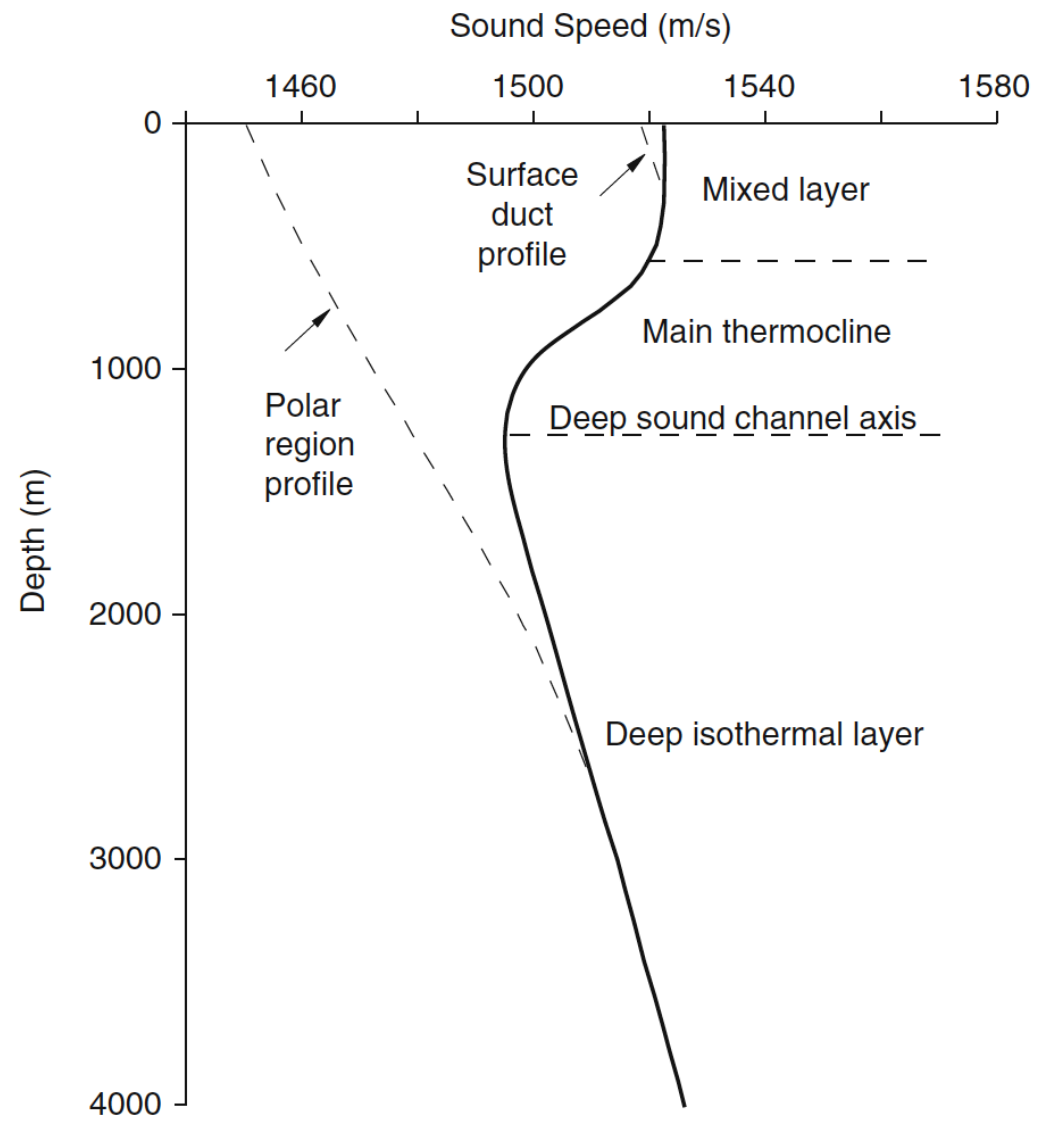

Figure 1-1: A Generic Sound Speed Profile. [3]

present at the surface in the Arctic. Those surface ice conditions are substantially different compared to the rest of the world's oceans. With the diminishing Arctic sea ice resulting from global warming the Arctic SSP is changing [1], [4], [5].

When looking at the differences in ray traces between a typical SSP and the historical Arctic, it's clear that the acoustic environment is quite different due to the ice cover and historically monotonically increasing SSP. The acoustic propagation paths we anticipate sound would travel in the Arctic are shown in Figure 1-2. These are the resulting ray traces expected from this SSP. Unlike Figure 1-3 which shows how a sound channel can result from the SSP in a typical deep ocean environment. Here a sound channel is present, and this enables sound propagation without any interactions from the surface or the bottom. When comparing the Arctic environment in Figure 1-2 with the typical deep ocean SSP in Figure 1-3 the acoustics in the Arctic tend to have far more surface interactions resulting in further scattering losses and signal attenuation [6], [3]. 


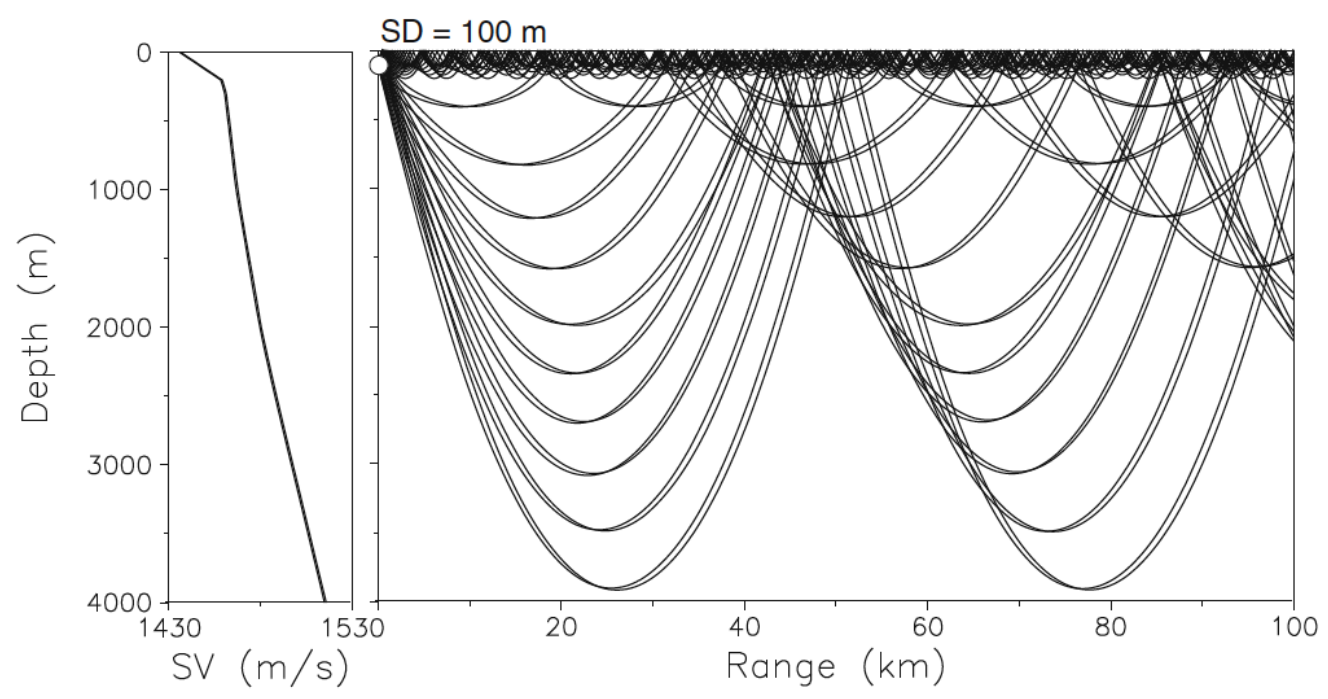

Figure 1-2: Arctic SSP with the propagation for a source depth of $100 \mathrm{~m}$. The ray diagram shows the sound doesn't interact with the bottom, but instead scattering occurs primarily from surface interactions. [3]

Often in ocean acoustics, a tool known as a CTD (Conductivity, Temperature, and Depth) is used to further extrapolate the in-situ SSP. The conductivity is directly related to the salinity of water, and the depth is a measurement of the pressure. This data is combined to determine the SSP of the water column. It's important to note that when deploying a CTD or other tool to understand the SSP, it only provides an instantaneous representation of the water column at that point in space and time. The SSP changes both spatially and temporally, however, I'll treat it as though it were spatially constant. Herein when modeling sound propagation throughout an area the same SSP is applied as range changes. I am however, comparing two different temporal SSPs with two simple model SSPs to evaluate the differences in anticipated acoustic propagation throughout the environment. The Expendable Conductivity Temperature Depth (XCTD) profiling system was used throughout ICEX, and the SSPs I'm going to focus on were taken using XCTDs.

\subsubsection{The Beaufort Lens}

The historical SSP model for the Arctic is no longer accurate. Instead of the simple monotonically increasing SSP that drives all sound upward back into the ice, an acoustic duct, or better known as the Beaufort Lens, is present between roughly 100 to $200 \mathrm{~m}$. Figure 1-4 shows the historical model for the Arctic environment vs the new SSP with the pres- 


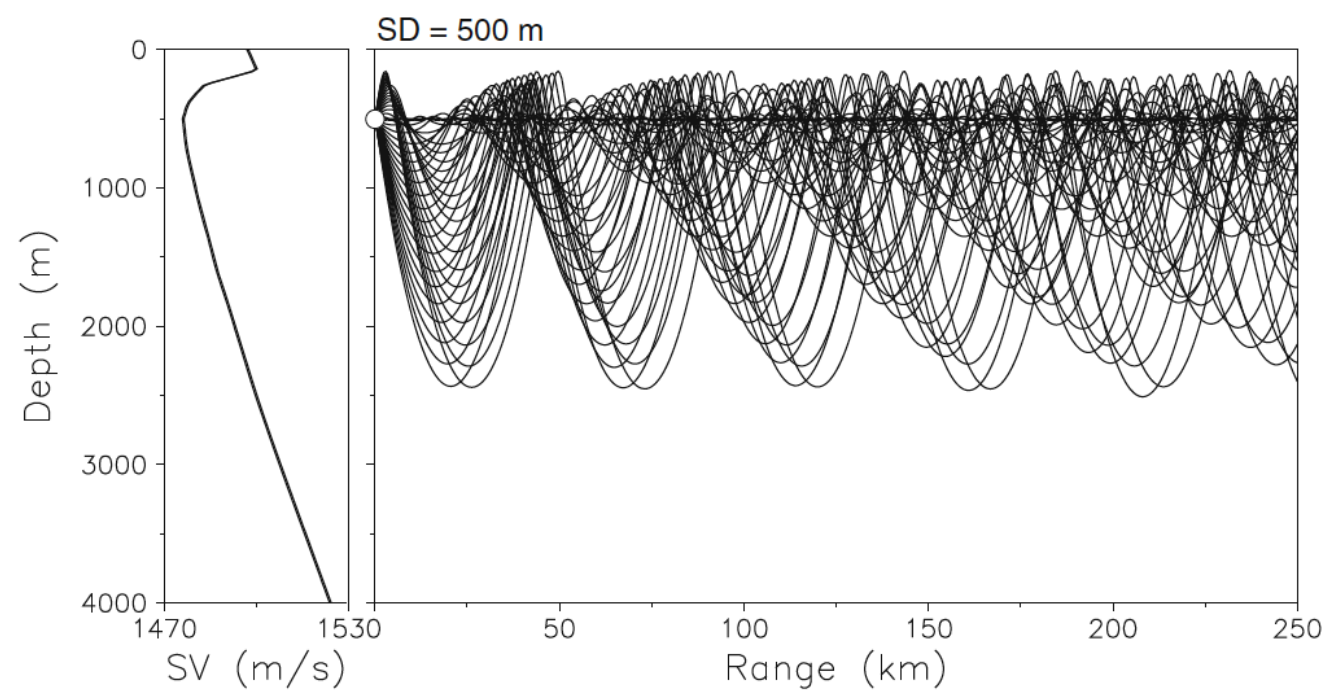

Figure 1-3: Typical deep water SSP with deep sound channel propagation. The source is at the depth of the deep sound channel axis [3].

ence of the Beaufort Lens. This creates the potential for trapping low frequency acoustic sound waves, enabling transmissions to travel greater ranges $(80-100 \mathrm{~km})$ without signal degradation from multiple surface interactions [5], [4].

During ICEX we wanted to validate the presence of the Beaufort Lens, map it's changes throughout our experiment, and understand it's impact on high frequency communications (10 kHz carrier frequency) employed as part of the navigation framework. Figure 1-4, compares an XCTD from 11-03-2020 at 1430UTC with the historical model of the Arctic environment. This XCTD was taken the morning of the un-tethered AUV operations, and was used to update the model used to approximate the environment. Looking at the XCTD cast, it's clear a unique feature exists roughly between 40 to $250 \mathrm{~m}$. This duct is the Beaufort Lens, and it was present with varying intensity throughout the experiment and our time on the ice.

Figure 1-5 shows the variability of the SSP throughout the course of a single day, 03-112020, when the majority of AUV operations occurred. It's clear from the plot on the left that the majority of the variability occurs between the surface and $100 \mathrm{~m}$. However, the Beaufort Lens is present in each of these XCTDs between 70 to $250 \mathrm{~m}$. The small duct between 30 and $70 \mathrm{~m}$ displays far more variability throughout the day and isn't always present, as seen in the 2100 AKDT XCTD. This XCTD displayed more small perturbations between 30 and $70 \mathrm{~m}$. The right plot, in comparison, shows that beneath $70 \mathrm{~m}$ there was little variability 


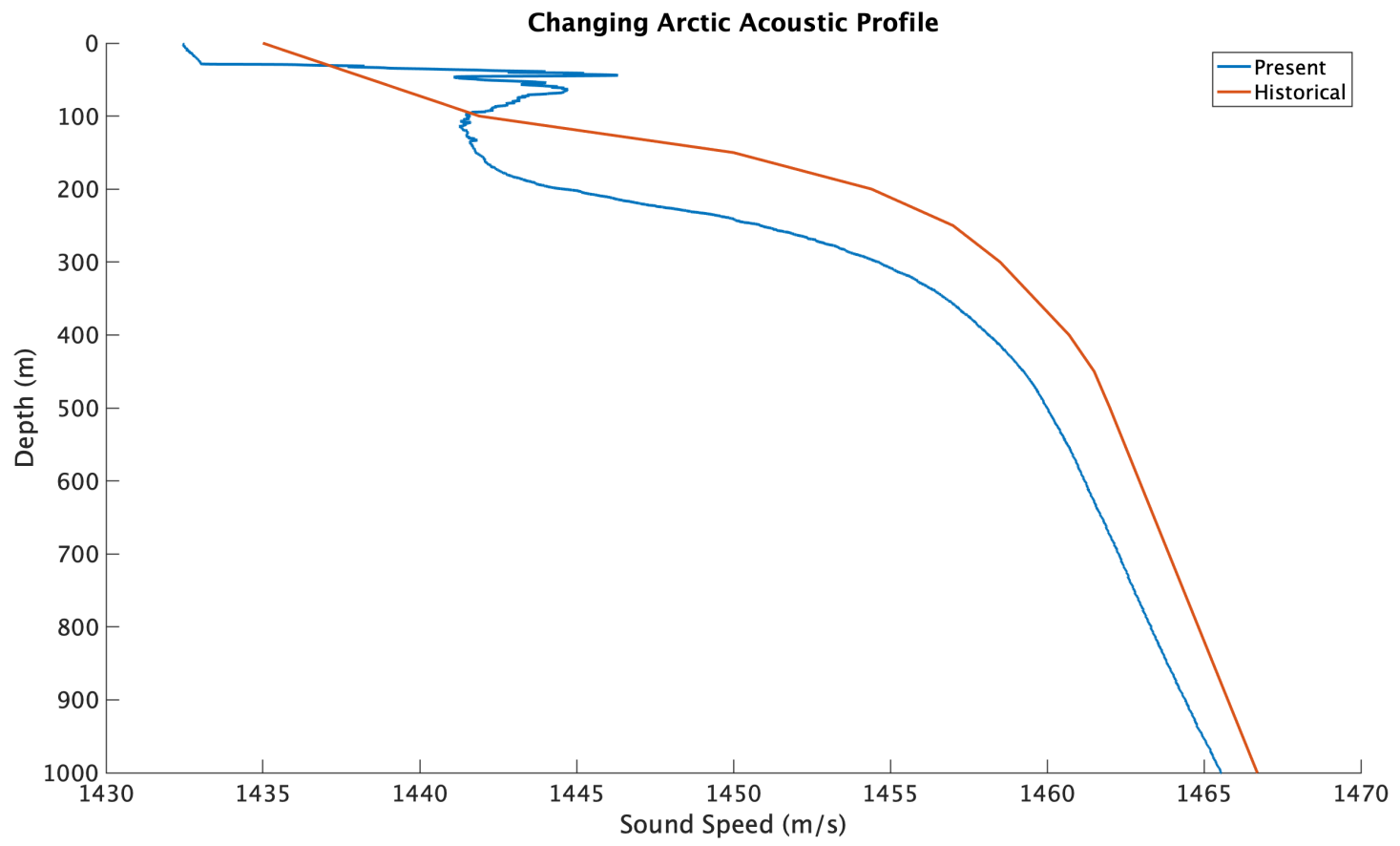

Figure 1-4: Historically monotonically increasing SSP as compared to the SSP present in the Arctic clearly showing the presence of the Beaufort Lens.

between the three XCTDs, and the SSP remains fairly constant there throughout the day. It is clear that the Beaufort Lens remains a prevalent acoustic feature in the Arctic Ocean. There is variability in the size and strength of the acoustic duct, but once below $70 \mathrm{~m}$ the duct was effectively consistent. This was true throughout our time on the ice in the Beaufort Sea.

\subsection{ICEX 2020 Experiment Design}

The experimental design for ICEX was quite complex, and there were a lot of moving parts. The full US Navy Exercise is three weeks long, conducted biennially, and involves coordination between multiple government agencies and the science community. The MIT/WHOI team was apart of the exercise that involved conducting autonomous undersea vehicle (AUV) operations with the Bluefin 21-inch diameter vehicle named "Macrura." Unlike previous AUV operations in the arctic, no dive team was on-site or employed to recover the AUV at the completion of its mission. During this operation, it was critical that the navigation system on-board Macrura maintained sufficient accuracy to enable the vehicle to navigate back to the "hydrohole" on its own. The hydrohole is a 3 foot by 15 foot hole cut through 

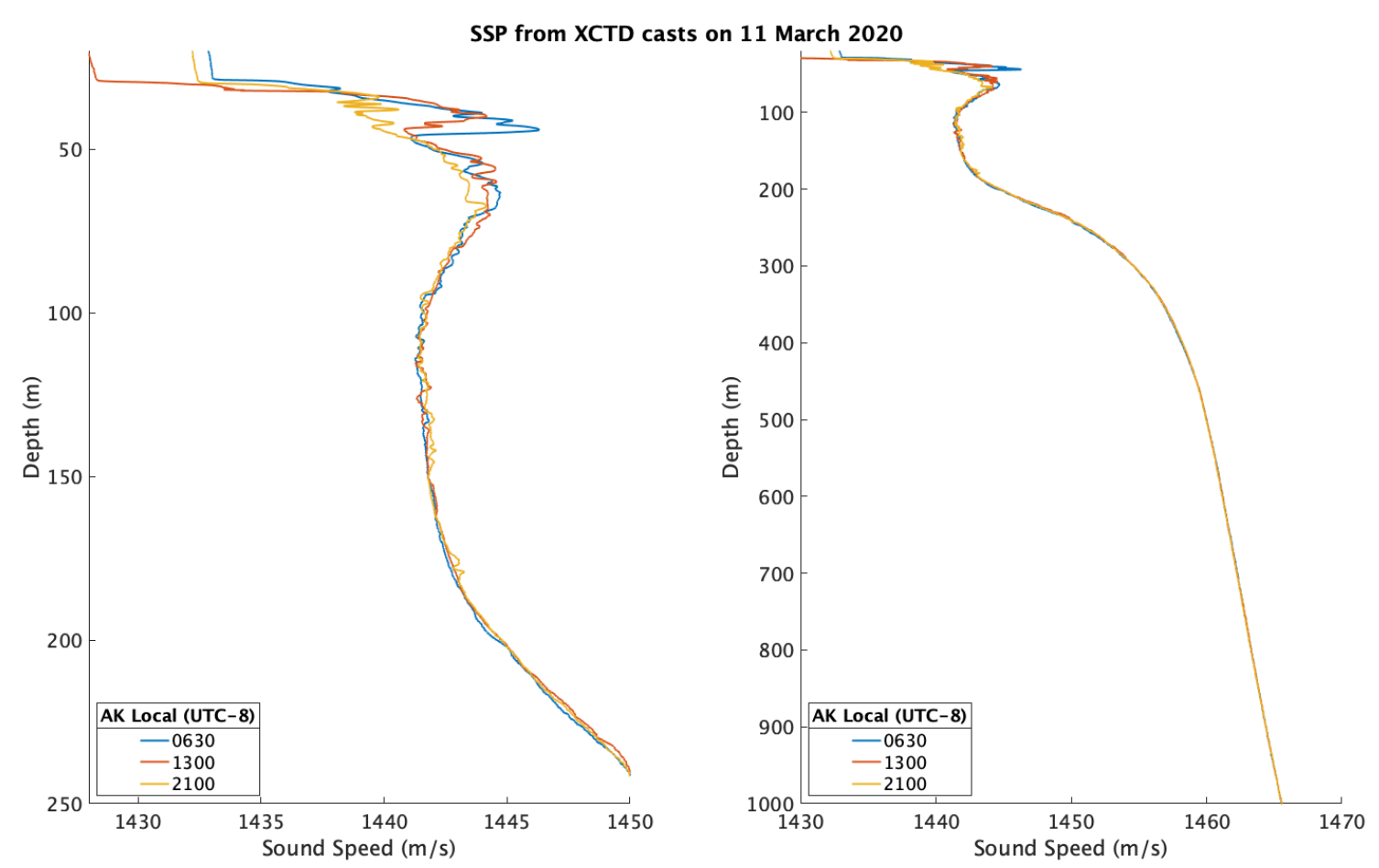

Figure 1-5: Variability of the SSP throughout a single day of AUV operations on the ice. These SSP are the raw XCTD data.

nearly 6 feet of ice in order to put Macrura in and out of the water. If the AUV didn't surface directly at the hydrohole, a small underwater, tethered, remotely operated vehicle (ROV) was employed to tie off Macrura for it to be pulled back into the hydrohole. Although Macrura may not surface through the hydrohole, the ROV was not meant to search for the vehicle. It was necessary to know where to drive the ROV in order to find Macrura, and the maximum range for vehicle recovery was approximately $50 \mathrm{~m}$ from the hydrohole. The navigation system needed to be quite accurate considering these constraints.

Unlike typical AUV operations, the Arctic offers significant challenges to navigation. Typical AUV operations have challenges navigating while submerged due to the rapid attenuation of electromagnetic waves in water [8], [9]. So continuous GPS positioning is unavailable for navigation, and AUVs carry their own onboard navigation systems. Typical AUVs surface throughout operations to receive a GPS fix and adjust their onboard navigation solution to improve or maintain accuracy. This isn't an option while conducting operations in the Arctic under the ice.

Other techniques to maintain accurate navigation solutions include the use of Doppler Velocity Logs (DVL) which improve an AUV's ability to accurately measure it's speed 


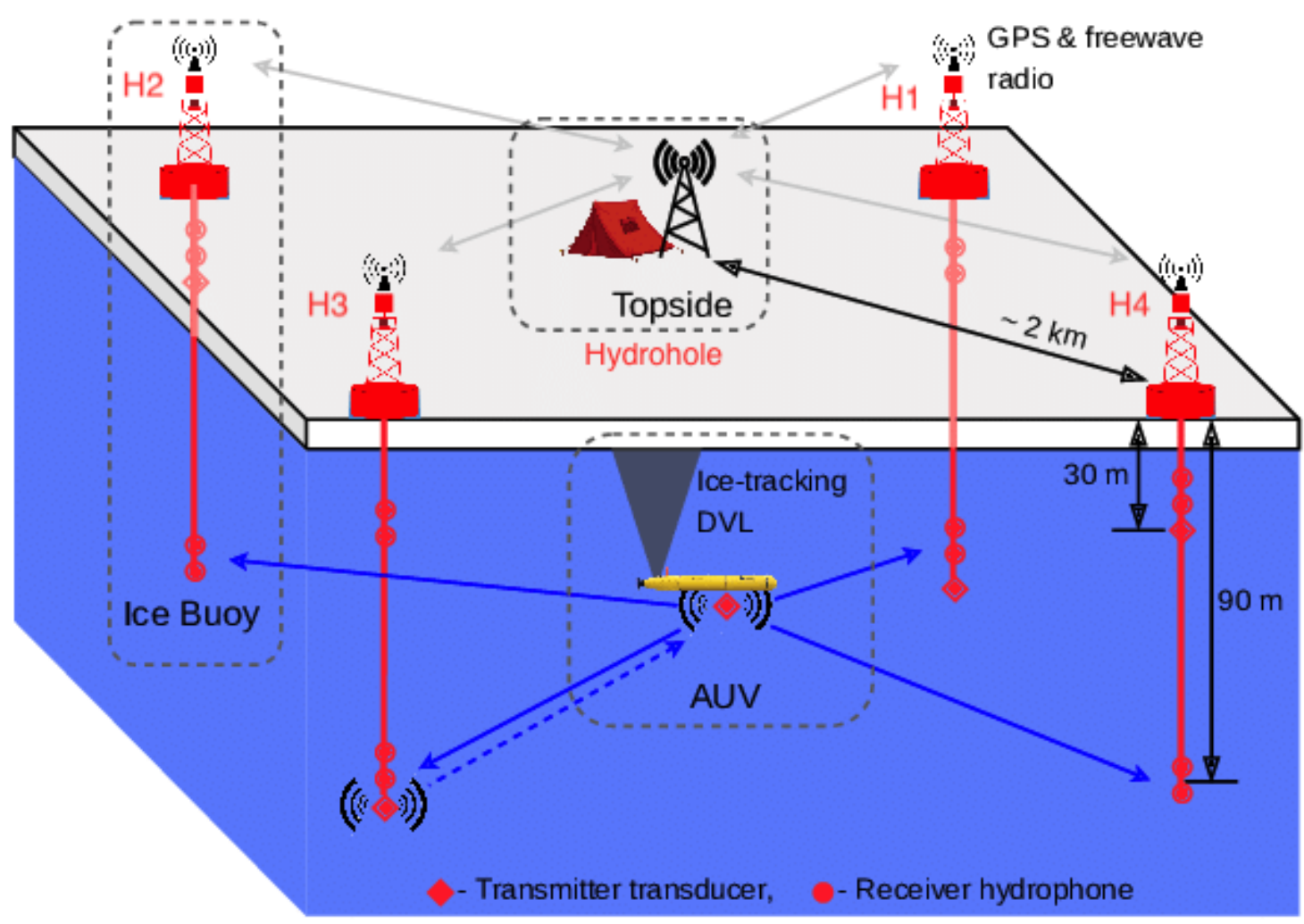

Figure 1-6: ICEX 2020 Experiment Layout. The hydrohole was located topside, where there was a large hole in the ice for the AUV to go in and out of the water. Each buoy included two shallow $(30 \mathrm{~m})$ and two deep $(90 \mathrm{~m})$ receivers, but only a single transmitter that was shallow or deep. [7]

over ground [10]. However, using a DVL for navigational purposes like this requires the AUV operating at a reasonable distance from the bottom. The Teledyne RDI Workhorse Navigator DVL was onboard Macrura for ICEX, and required operating less than $200 \mathrm{~m}$ from the bottom [11].

Based on planned operations in the Beaufort Sea, where depth was in excess of $2000 \mathrm{~m}$, and Macrura's operating depth restriction of $200 \mathrm{~m}$, a standard DVL approach to navigation was not feasible. The DVL was inverted to transmit upward. Typically this does not work well since wave motion at the surface prevents the DVL from making accurate Doppler measurements used for navigation purposes [12]. Upward facing DVLs are used more broadly as Acoustic Doppler Current Profilers (ADCP) to measure the current throughout the water column.

Due to the ice cover in the Arctic, flipping the DVL provided a more stable solution than open ocean operations. Keep in mind, the ice is also moving, but typically at much slower 
speeds than normal wave motion. To account for this, topside communicated the ice motion to the AUV. This allowed Macrura's navigation calculation to account for the Doppler from the ice, and not just take the DVL speed as ground truth.

Figure 1-6 also shows the WHOI micro-modems that were used. Sometimes they're referred to as buoys, or more specifically as H1, H2, H3, and H4. These buoys were placed in the ice with hydrophones at $30 \mathrm{~m}$ and $90 \mathrm{~m}$. Each buoy was equipped with a single transducer at either $30 \mathrm{~m}$ (H2 \& H4) or $90 \mathrm{~m}$ (H1 \& H3) for transmitting.

NOTE: Buoys H1, H2, H3, \& H4 can only receive at one depth of hydrophones at any given time. So the buoys are set to receive shallow or deep, but cannot receive shallow and deep at the same time, even if a transmission were to reach both shallow and deep hydrophones, only the currently selected hydrophone reports the reception. Even though there are two hydrophones at each depth for receiving, the WHOI micro-modem doesn't make the distinction between the two hydrophones.

\subsubsection{DURIP Towed Array}

The physical layout and design of the DURIP towed array is shown in Figure 1-7. The DURIP towed array uses a sampling frequency of $12 \mathrm{kHz}$, and is comprised of 32 elements. 21 Elements are spaced at $1.5 \mathrm{~m}$, and 23 are spaced at $0.75 \mathrm{~m}$.

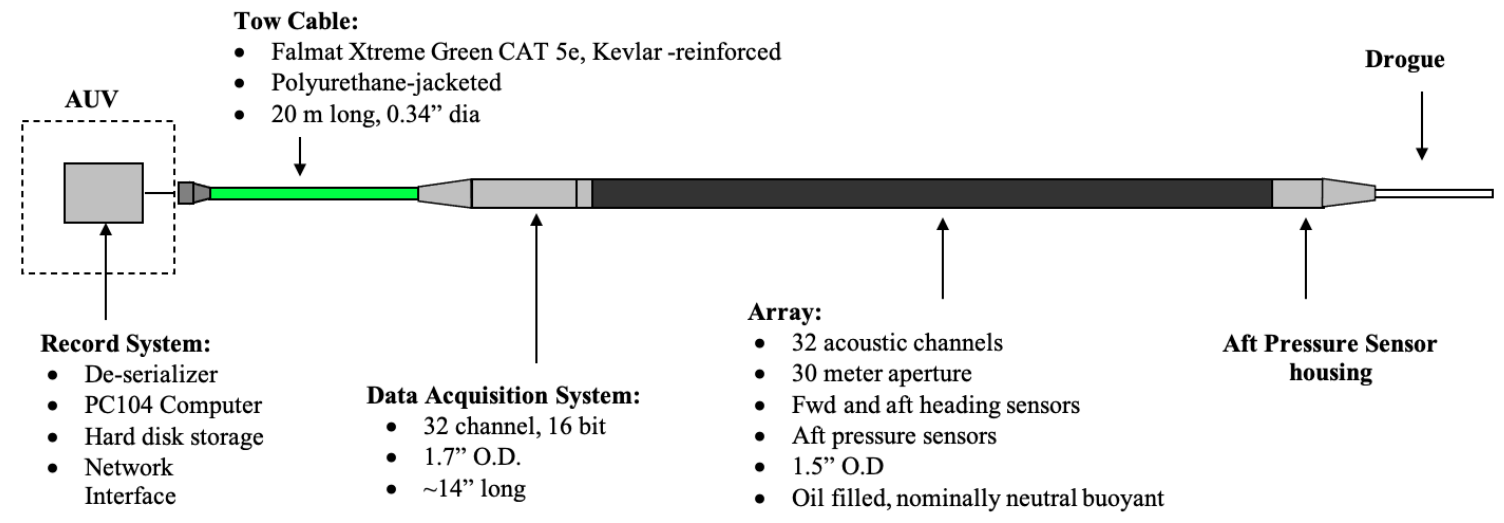

Figure 1-7: Physical design of DURIP towed array.

The element layout of the array is quite unique as shown in Figure 1-8. It's considered a nested array. The beamforming implications are discussed further in chapter 4 , however it's important to note that the full aperture of the array $(30 \mathrm{~m})$ is cut for $500 \mathrm{~Hz}(1.5 \mathrm{~m}$ element spacing), and the smaller nested array is cut for $1000 \mathrm{~Hz}$ (0.75 m element spacing). 


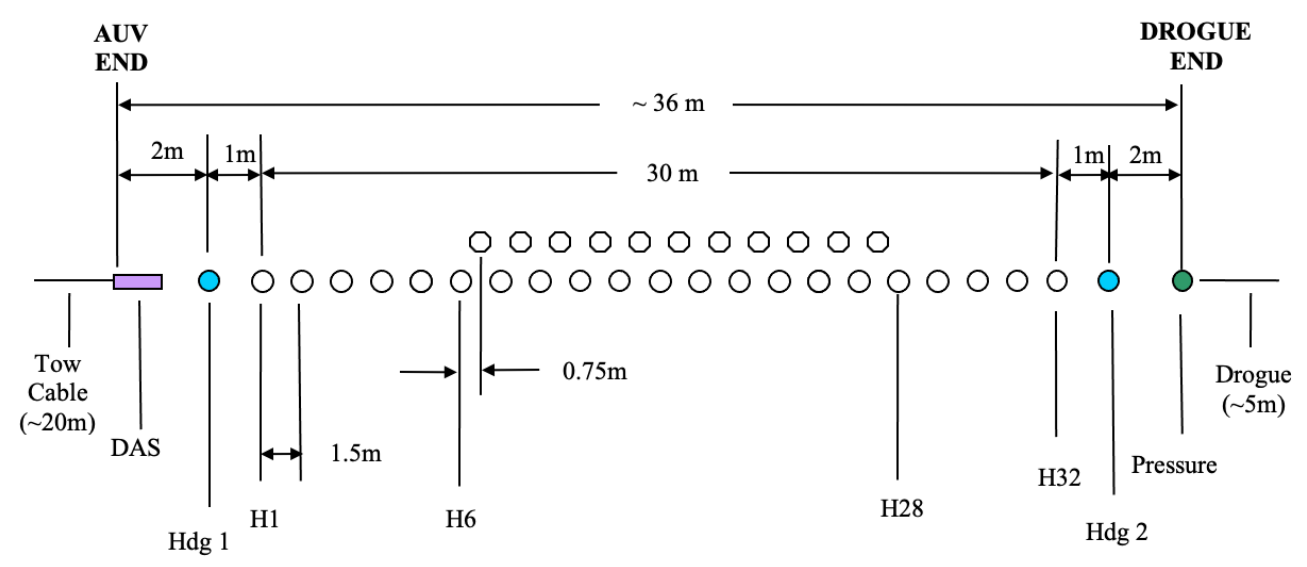

Figure 1-8: DURIP towed array internal element layout.

To prevent aliasing, discussed further in Chapter 2, which occurs for all frequencies > $f_{\text {Nyquist }}=6 \mathrm{kHz}$, a low-pass filter is included in the Digital Acquisition System (DAS) for the DURIP towed array. Figure 1-9 shows the block diagram of the DURIP towed array DAS and how it connects to the AUV. In the middle of the DAS is the Analog to Digital (A/D) converter (ADC) that includes a 5 delta sigma filter. The filter prevents nearly all frequency content above $6 \mathrm{kHz}$, the Nyquist frequency, from being sampled and converted into digital data. As a result, the $10 \mathrm{kHz}$ WHOI micro-modem transmissions are not sampled by the DURIP towed array even though the sampling frequency is $12 \mathrm{kHz}$. Instead the ADC is effectively only sampling frequencies less than $6 \mathrm{kHz}$.

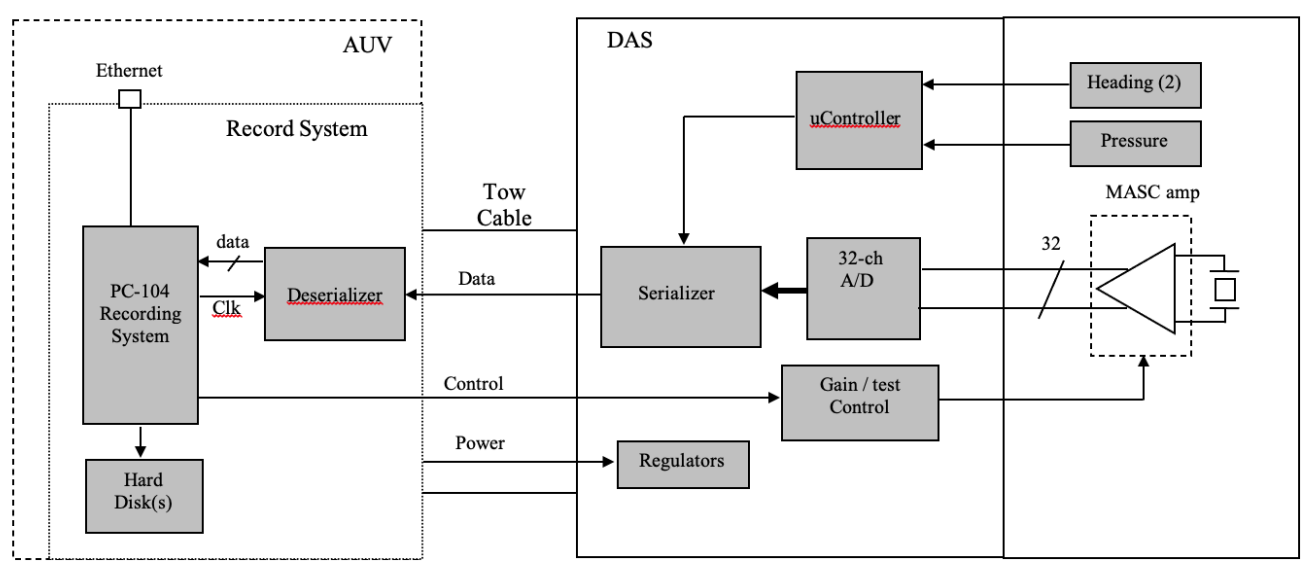

Figure 1-9: Block Diagram for DURIP towed array and connections to Macrura.

This anti-aliasing filter prevents noise and sources of sound $>6 \mathrm{kHz}$ from interfering with the DURIP towed array's recordings, and the post-processing of towed array data is 
actually indicative of the $0-6 \mathrm{kHz}$ spectrum that the array elements are designed to record.

\subsubsection{Harmonics and Subharmonics}

When an acoustic sound is produced, there are also harmonics that are produced. For example, a $50 \mathrm{~Hz}$ tonal may have an associated $100 \mathrm{~Hz}$ and $150 \mathrm{~Hz}$ tonal too. These are the harmonics from the originally produced $50 \mathrm{~Hz}$ sound. Although harmonics often refer to frequencies that are multiples above the originally produced sound wave, subharmonics that can also be produced.

In our particular case we were attempting to use the DURIP towed array to record some of the acoustic signals transmitted by the WHOI micro-modems. Since an anti-aliasing filter was in-place, to prevent noise $>6 \mathrm{kHz}$ from aliasing, another mechanism for recording the 10 $\mathrm{kHz}$ acoustic modem transmissions was required. Due to the make-up of the GeoSpectrum M27-930 Free Flooding Ring (FFR) transducers [13], a subharmonic is produced at $5 \mathrm{kHz}$ when transmitting acoustic signals at $10 \mathrm{kHz}$.

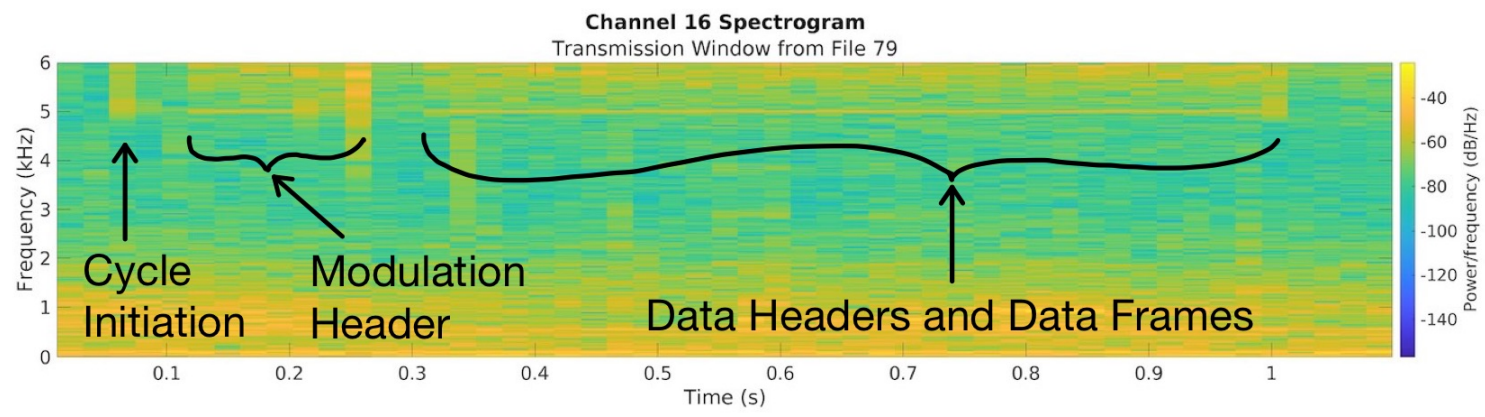

Figure 1-10: Spectrogram of a WHOI micro-modem transmission as recorded by element 16 from the DURIP towed array.

Figure 1-10 looks at the spectrogram of a WHOI micro-modem transmission. There is a discrete line at $5 \mathrm{kHz}$ and additional noise present between $5-6 \mathrm{kHz}$ throughout the transmission. This enabled the DURIP towed array elements to record acoustic transmissions from the WHOI micro-modems even though the actual transmission frequency was $10 \mathrm{kHz}$ and above the Nyquist frequency and filter cutoff frequency of $6 \mathrm{kHz}$. Since the $5 \mathrm{kHz}$ subharmonic was below the cutoff frequency and less than the Nyquist frequency, its frequency content was captured by the recording elements of the towed array and would provide an opportunity to potentially beamform, which is discussed further in chapter 4. Figure 1-10 shows the different portions that make up the WHOI micro-modem transmissions. The 
transmission is comprised of three portions: the cycle initiation; modulation headers; and data headers and data frames.

\subsubsection{Power Spectral Density throughout the Vertical Run}

One operations period that I'll refer to throughout this paper is the "Vertical Run." This occurred the morning of 03-11-2020 (1655-1830UTC). During this operations period, Macrura was tethered to the hoist inside the hydrohole, and the AUV was stationary. The AUV was held by two lines connected from the hoist to the vehicle at the forward and aft mounts. Figure 1-11 shows the depth profile for Macrura throughout this operations period, and includes the transmissions made from Macrura, H1, and H2. Based on background noise (other sources in the water, topside generators, snowmobiles, and naturally occurring environmental noise), not all of the buoy transmissions were easily discernible. The blue circles indicate the specific buoy transmissions that were used here to calculate the mean Power Spectral Density (PSD).

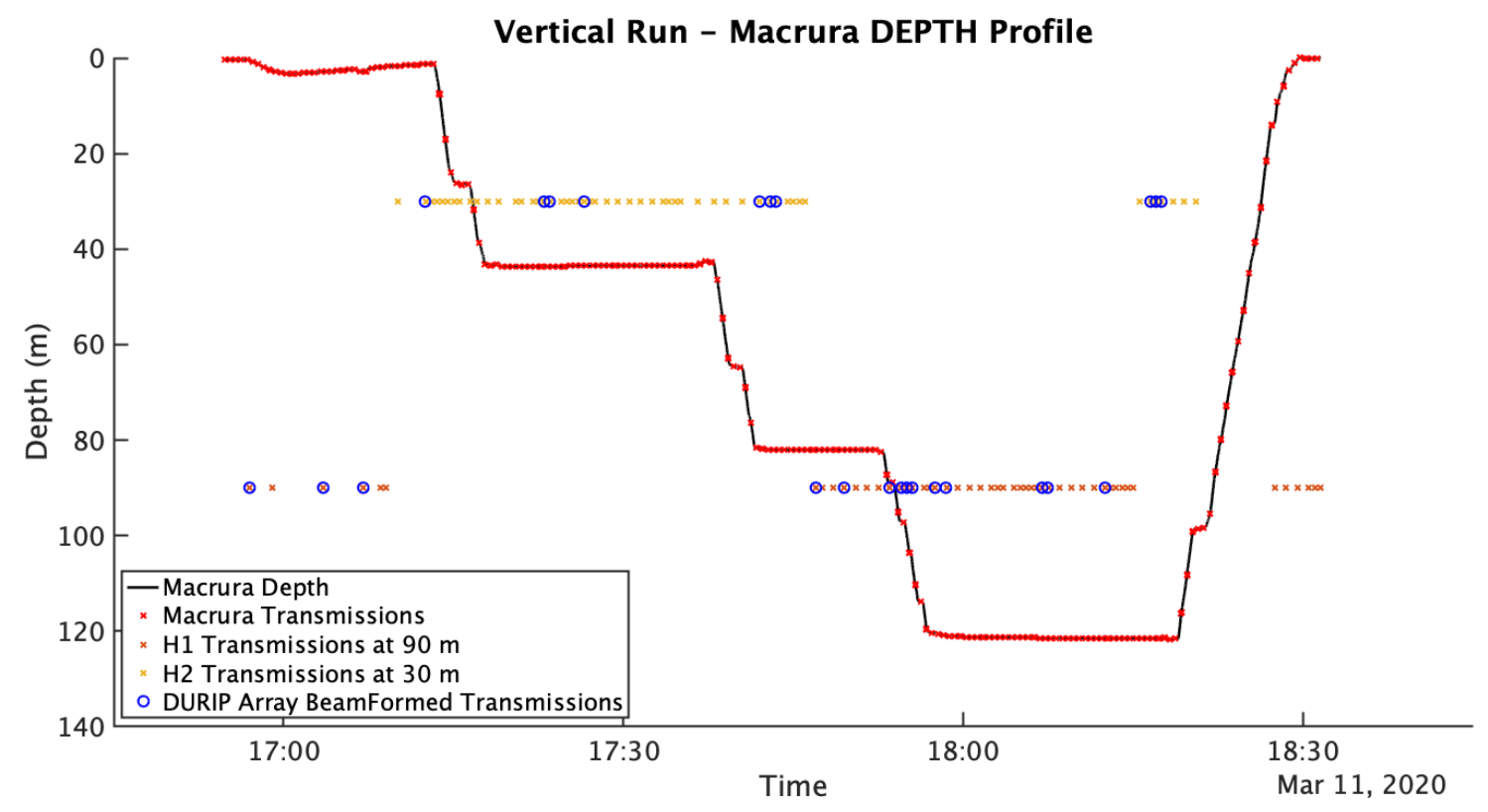

Figure 1-11: Vertical Run depth profile for Macrura. The vehicle was not moving during this time-period, and the transmissions from $\mathrm{H} 1$ at $90 \mathrm{~m}$ and $\mathrm{H} 2$ at $30 \mathrm{~m}$ are present.

During the vertical run, the DURIP towed array was employed with a weight tied to the end to hold the tail end of the towed array down so it was acting as a vertical line array that wasn't moving. With the DURIP towed array in this vertical configuration, it is roughly $38 \mathrm{~m}$ deeper to the center of the array from the vehicle's depth. This was the 
first in water check for the DURIP towed array during ICEX. Moreover it was a perfect opportunity to test the implications of the Beaufort Lens on the high frequency $(10 \mathrm{kHz})$ WHOI micro-modem communication transmissions and validate the acoustic paths.

A top-down view of the layout is shown in Figure 1-12. It's important to note, that because the Hydrohole and buoys (H1, H2, H3, \& H4) are all located on the ice, they're all moving and not necessarily in the same direction or speed. Conveniently throughout the Vertical Run there was little ice motion, and the buoys and Hydrohole maintained their relative positions.

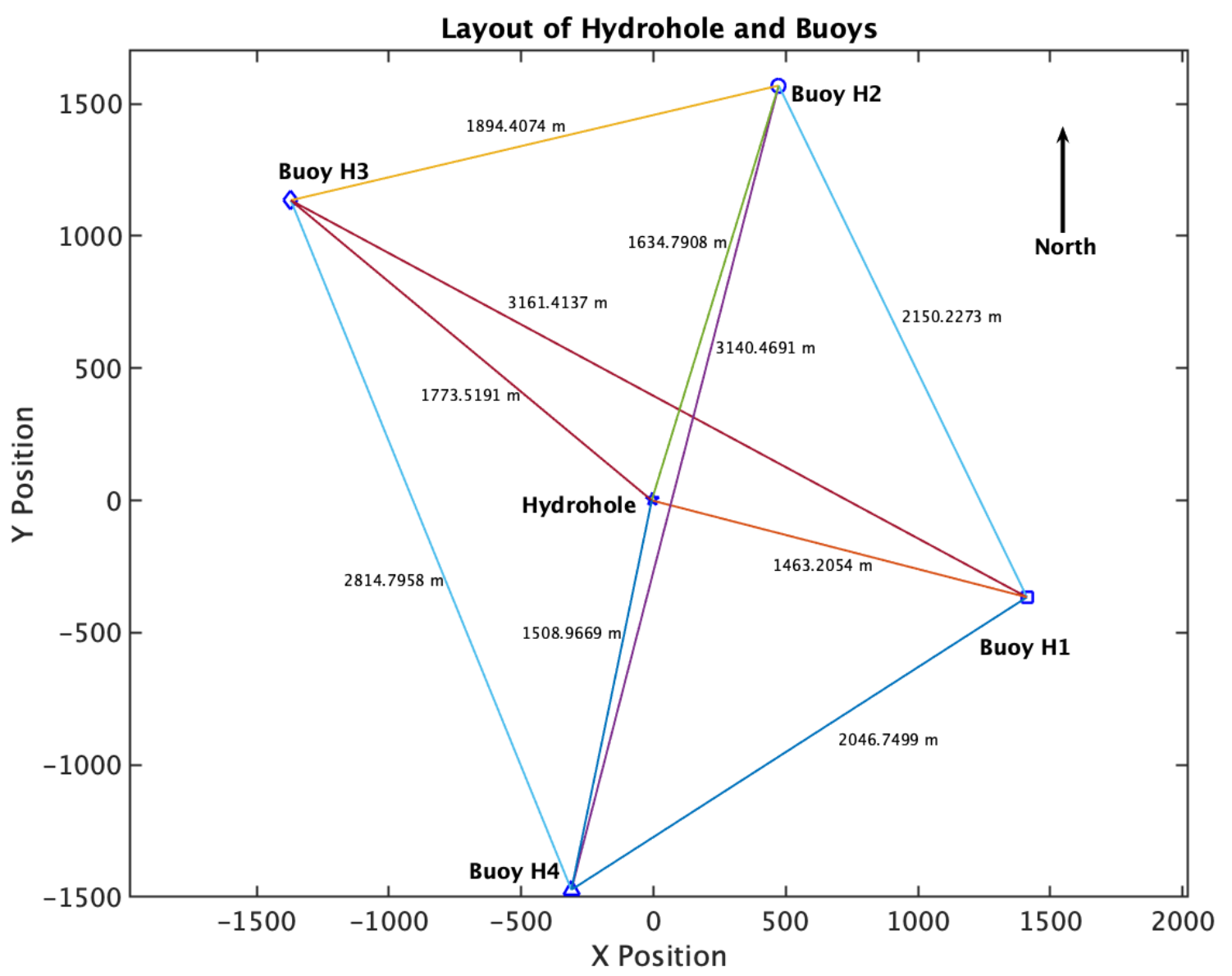

Figure 1-12: Top down view of Hydrohole and buoy locations with ranges

In order to ensure the $5 \mathrm{kHz}$ sub-harmonic was present during multiple transmissions from the WHOI micro-modem, and on all 32 elements on the DURIP towed array, I evaluated the PSD throughout the Vertical Run. The blue circles in Figure 1-11 are the transmissions from buoys $\mathrm{H} 1$ \& $\mathrm{H} 2$ that I evaluated. These transmissions were from the WHOI micromodems and clearly visible on the DURIP towed array. Additionally, the Vertical Run was 
a unique geometry since the array was going through the Beaufort Lens, and the sources, Buoy $\mathrm{H} 1$ and Buoy $\mathrm{H} 2$ were in the Beaufort Lens at $90 \mathrm{~m}$ and in the surface duct at $30 \mathrm{~m}$ respectively. This was perfect for analyzing the environmental impacts on high frequency acoustic communications.

I looked at one second prior to receiving the transmission on the DURIP towed array to establish a background PSD based on the noise present at each element immediately preceding the buoy transmission arriving at the DURIP towed array. I compared that to the PSD of each element during the receipt of the buoy transmission. Figure 1-13 shows this analysis.

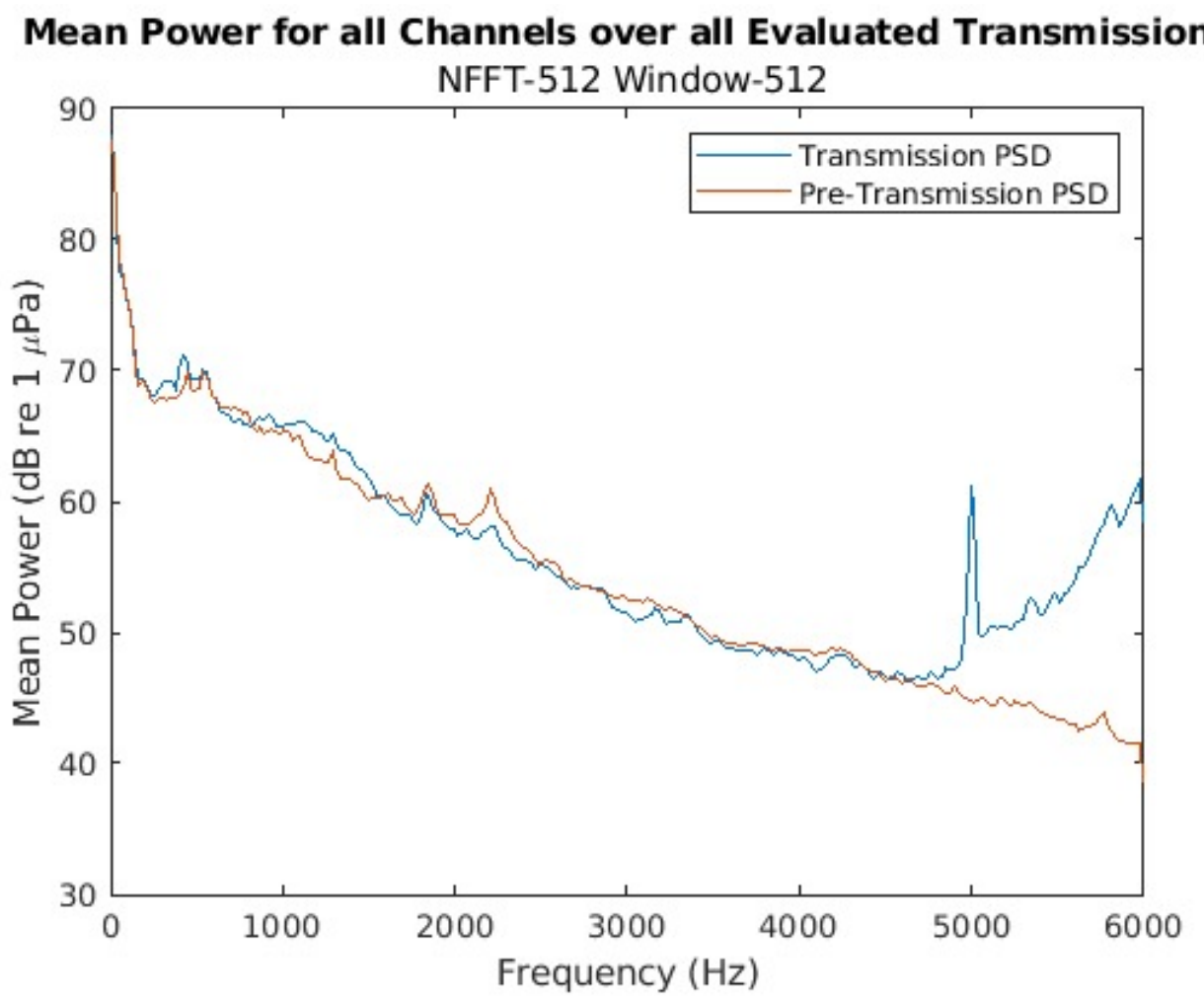

Figure 1-13: Power Spectral Density (PSD) for all elements on the towed array averaged together over multiple buoy communication transmissions from the WHOI micro-modems.

The mean PSD clearly shows the $5 \mathrm{kHz}$ was a dominant feature throughout the Vertical Run. As well, its clear that between $5-6 \mathrm{kHz}$, the mean power is diverging significantly from the background noise. Figure 1-14 shows the increase in source level that occurs between $5-6 \mathrm{kHz}$ for the WHOI micro-modem transducers. This is the reason the mean PSD diverges from the background noise between 5-6 kHz. Although this isn't the exact frequency of the WHOI micro-modem transmission, the additional source level assists in the beamforming 
analysis in chapter 4 .

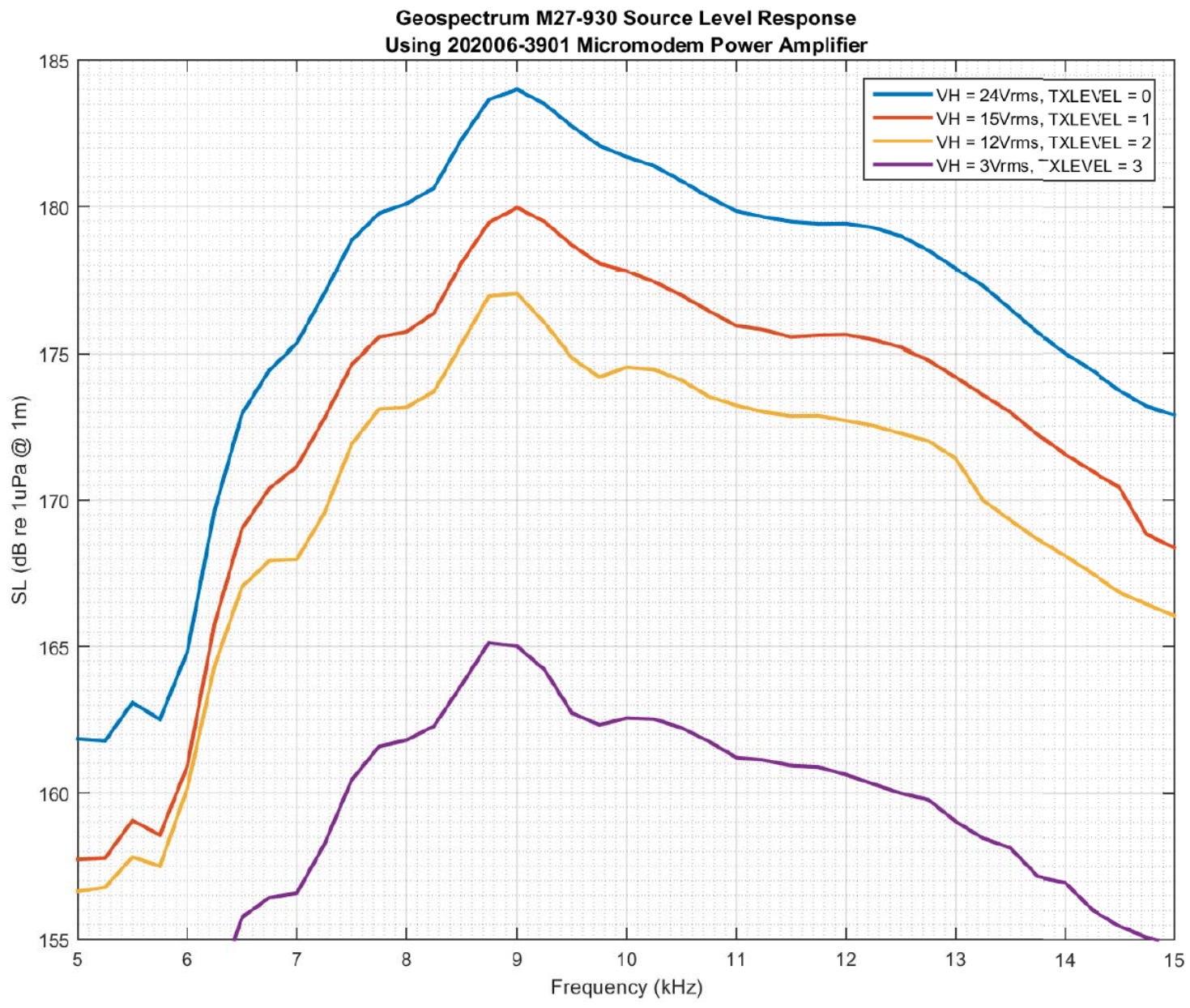

Figure 1-14: WHOI micro-modem transducer source level. This is the transducer that was used on the buoys (H1, H2, H3, \& H4) during ICEX for transmitting. During ICEX the transducer was set to the blue line, $\mathrm{VH}=24$ Vrms. 


\section{Chapter 2}

\section{Background in Fundamentals of \\ Ocean Acoustics and Digital Signal Processing with Beamforming}

Throughout the paper, some fundamentals are discussed. The majority of the concepts are covered more in-depth throughout the textbook Computational Ocean Acoustics, [3] and Optimum Array Processing, [14]. It's important to have a functional understanding of these ideas which are pervasive throughout the research discussed herein. I'll be discussing sound waves, their propagation throughout water, and acoustic beamforming on a linear array to determine directionality.

\section{LONGITUDINAL WAVES}

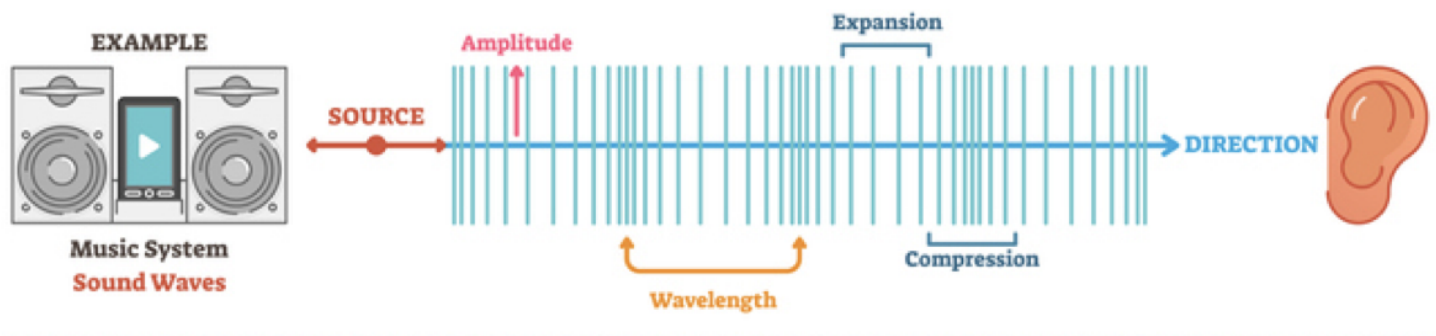

Figure 2-1: Longitudinal sound wave. How sound propagates. [15] 


\subsection{Ocean Acoustics and Sound Waves}

Sound waves are longitudinal waves unlike electromagnetic or other common wave forms that are transverse. A typical wave easily visualized is a calm pond with a pebble dropped into the middle of it. The resulting waves travel outward in circular patterns from the point where the rock disrupted the calm waters. The particles of water are moving up and down, but the wave moves perpendicular to that motion, outward from the original location where the pebble fell. Unlike this type of motion, sound is a longitudinal wave. Similar to the transverse wave, the particles only move slightly, but instead of perpendicular to the direction of wave motion, particles move parallel to the direction of wave motion. Figure 2-1 shows how sound waves are longitudinal and move.

\subsubsection{The Wave Equation}

Due to the nature of acoustic sound waves traveling through a medium as a longitudinal wave, pressure is the means with which we measure sound waves. The hydrophones employed by the WHOI micro-modem buoys and the DURIP towed array elements are tools used to measure slight pressure disturbances in the ocean environment. Mathematically we describe the wave equation by combining the ideas of conservation of mass, and Newton's second law of motion. The linear approximation only involves the first order terms and is written as follows:

$$
\nabla^{2} p-\frac{1}{c^{2}} \frac{\partial^{2} p}{\partial t^{2}}=0
$$

Here $\nabla^{2}$ represents the Laplace operator or the divergence of the gradient. In Cartesian coordinates it would be described as $\nabla^{2}=\frac{\partial^{2}}{\partial x^{2}}+\frac{\partial^{2}}{\partial y^{2}}+\frac{\partial^{2}}{\partial z^{2}}$. P is the pressure, and $\mathrm{c}$ represents the speed of sound in the medium (ie. speed of propagation). In our case the medium is the ocean, and the speed of sound is not constant. Instead, the speed of sound changes both spatially and temporally and is represented by the SSP.

The acoustic wave number, $k$ is another important relationship to remain aware of:

$$
k=\frac{2 \pi}{\lambda} \quad \text { or } \quad k=\frac{\omega}{c} \quad \text { where } \quad \omega=2 \pi f \quad \text { and } \quad f=\frac{c}{\lambda}
$$

The acoustic wavenumber is used because it helps simplify many equations later on. The acoustic wavenumber captures the relationship between density, speed of sound, frequency, 
and wavelength.

\subsubsection{Snell's Law and Ray Tracing}

Ray tracing is a technique that is used to model the propagation paths or trajectories of sound waves in a medium. The ray path is a vector that is drawn perpendicular to the wave front as in Figure 2-2a. These basic ray paths are drawn without any bending, although

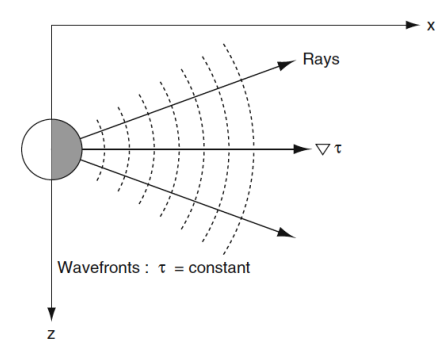

(a) Ray paths are perpendicular to the wave front.

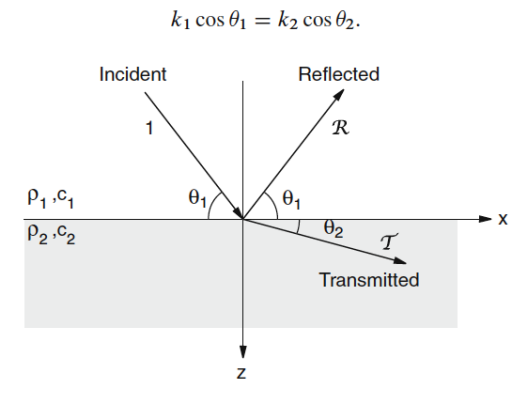

(b) Snell's Law

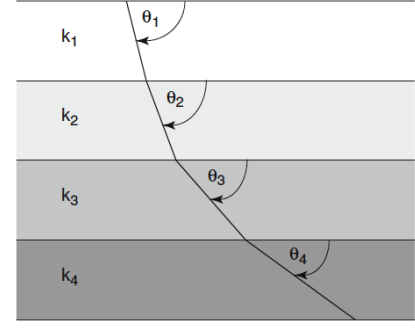

(c) Rays refract through mediums according to Snell's Law

Figure 2-2: Ray paths, combined with Snell's Law enable ray tracing. This provides an approximation for how sound waves propagate through a medium and the trajectories the sound waves follow. This also means that the wave front bends. [3]

that isn't always the case. Snell's Law of refraction $k_{1} \cos \left(\theta_{1}\right)=k_{2} \cos \left(\theta_{2}\right)$ describes how a ray refracts or "bends" when it interacts with a medium of different density as described by the acoustic wavenumber, $k$. Figure $2-2 \mathrm{~b}$ shows how this refraction takes place according to Snell's Law.

Another way to think about Snell's Law is that $\frac{\cos (\theta)}{c}=$ a constant. This is another way to say that if the SSP throughout the water column were isovelocity (a vertical line), the ray trace would not bend at all. The initial trajectory of the acoustic wave would remain constant until it came into contact with either the surface or the bottom, at which point surface scattering and bottom scattering would occur. However, the SSP isn't isovelocity and changes throughout the water column. As a result, the wavenumber changes throughout the water column. Changes in the wavenumber throughout the water column result in the ray paths bending up and down. Most importantly, the ray paths bend toward regions of low sound speed. This is why acoustic ducts, or channels are located at minimums in the SSP. This is also true of the Beaufort Lens.

Unlike in Snell's Law diagram in Figure 2-2b, when we refer to the ray paths throughout the water column the reflected waves are neglected as shown in Figure 2-2c. This is largely 
due to the relationship between the reflection, $\mathcal{R}$ and transmission, $\mathcal{T}$ coefficients:

$$
\mathcal{R}=\frac{\mathcal{Z}_{2}-\mathcal{Z}_{1}}{\mathcal{Z}_{2}+\mathcal{Z}_{1}}, \quad \text { and } \quad \mathcal{T}=\frac{2 \mathcal{Z}_{2}}{\mathcal{Z}_{1}+\mathcal{Z}_{2}} \quad \text { where } \quad \mathcal{Z}_{i}=\frac{\rho_{i} c_{i}}{\sin \left(\theta_{i}\right)}
$$

When $\rho_{1} c_{1} \approx \rho_{2} c_{2}$, the resulting value for $\mathcal{R} \rightarrow 0$, and $\mathcal{T} \approx 1$. This means the portion of the wave that is reflected while moving through the medium is nearly zero, and the transmitted portion of the wave dominates. This is why ray tracing disregards the reflection, and focuses on the transmitted portion of the wave, with the exception of at the boundary conditions (eg. the surface and bottom). At the surface and bottom, the reflection dominates, and the transmission coefficient is much lower.

Figure 2-2c shows how the ray bends as it goes through different portions of the water column. Each layer would be akin to a small change in the SSP. Over a longer period of time, this would look similar to Figure 1-2 and Figure 1-3. These plots show the anticipated propagation paths or ray paths for sound waves in that medium with the associated SSP.

NOTE: The SSP changes both spatially and temporally. We saw this in Figure 1-5. While discussing ray tracing we're treating the spatial and temporal perturbations in the SSP as non-existent. This means, the SSP that we apply to determine the ray paths is applied equally in range and time. However, we know from real-world experience that this isn't the actual case.

\subsubsection{Transmission Loss}

As acoustic sound waves traverse the oceans, they undergo losses. Typically these losses are largely comprised of geometric losses from spreading, and attenuation. At the heart of geometric spreading is conservation of energy. Consider a point source putting out energy into the water. It cannot produce the same amount of energy $100 \mathrm{~m}$ from it in all directions. Instead, the energy is dissipated over the larger surface area.

More accurately transmission loss is a comparison of the intensity, $I$. This is the average rate of energy flow through a unit area perpendicular to the direction of wave propagation.

$$
I=\frac{\mathcal{P} \mathcal{V}}{2}=\frac{p_{r m s}^{2}}{\rho_{o} c} \quad \text { where } \quad p=\mathcal{P} e^{j \omega t+\theta} \text { and } v=\mathcal{V} e^{j \omega t+\theta}
$$

Although p, the pressure, and $\mathrm{v}$, the velocity can be represented in complex fashion to 
describe their wave behavior, intensity is real, so it's the average over one period, $T$.

$$
I=\frac{1}{T} \int_{0}^{T} \mathcal{P} \mathcal{V} \cos (\omega t+\theta) d t=\frac{1}{2} \operatorname{Re}\left\{p^{*} v\right\}
$$

In underwater acoustics we describe transmission loss using a dB scale with the standard measurement, $I_{o}$, is taken at $1 \mathrm{~m}$ from the source. The resulting calculation is as follows:

$$
\mathrm{TL}=-10 \log \frac{I(r, z)}{I_{o}}
$$

So the intensity at some given position in the field, $(\mathrm{r}, \mathrm{z})$ compared to the intensity $1 \mathrm{~m}$ from the source. Often measurements are taken in units of pressure, so this can also be converted:

$$
\mathrm{TL}=-10 \log \frac{p(r, z)^{2}}{p_{o}^{2}}=-10 \log \left(\frac{|p(r, z)|}{\left|p_{o}\right|}\right)^{2}=-20 \log \frac{|p(r, z)|}{\left|p_{o}\right|}
$$

Due to the nature of geometric spreading in the ocean the two most common scenarios are spherical spreading, which occurs closer to the source, and as you move further away from the source the constraints of the ocean surface and the bottom dominate, and we transition to cylindrical spreading. In each of these cases the intensity is inversely proportional to the surface area. So spherical spreading loss is $I \propto 1 /\left(4 \pi r^{2}\right) \Rightarrow T L=20 \log (r)$ and the cylindrical spreading loss is $I \propto 1 /(2 \pi r D) \Rightarrow T L=10 \log (r)$, where $D$ represents the total water depth. So while $r \leq D$, there is spherical spreading, then there's a transition period until $r>>$, when its cylindrical spreading.

Transmission loss is often preferred as an evaluation metric compared to basic ray tracing because it includes the degradation that a signal undergoes as it propagates through the water. In my analysis I was less concerned with transmission loss, and focused on the reliable acoustic path the ray followed to arrive at Macrura successfully.

\subsubsection{Attenuation}

Acoustic waves undergo attenuation as they propagate throughout the ocean. These losses are modeled as an exponential decay:

$$
A=A_{o} e^{-\alpha x}
$$


$A_{o}$ is the initial amplitude at $x=0$, and $\alpha$ represents the plane-wave attenuation. So a plane-wave undergoing attenuation is described as: $e^{j k x-\alpha x}$ and recall $\mathrm{k}$ is the wavenumber from Equation 2.2. The loss is most frequently described in units of decibels $(\mathrm{dB} / \mathrm{m}), \alpha^{\prime}$.

$$
\text { Loss }=-20 \log \left(\frac{A}{A_{o}}\right)=-20 \log \left(\frac{A_{o} e^{-\alpha x}}{A_{o}}\right)=20 \log (e) \alpha x \approx 8.686 \alpha x \Rightarrow \alpha^{\prime} \approx 8.686 \alpha
$$

Attenuation can also be described as a function of frequency in units $\mathrm{dB} /(\mathrm{m} \mathrm{kHz})$ or wavelength in units of $\mathrm{dB} /$ wavelength:

$$
\alpha^{(f)} \approx 8686 \frac{\alpha}{f} \quad \text { or for wavelength } \quad \alpha^{(\lambda)}=\alpha \lambda 8.686
$$

Fundamentally, attenuation is dependent upon frequency, and as frequency increases, attenuation does as well. Although this relationship appears linear, it's actually a bit more complex as shown in Figure 2-3. Most notably, higher frequencies attenuate quicker, and the WHOI micro-modem is transmitting at $10 \mathrm{kHz}$. Further research found a more complicated formula that attenuation due to temperature and pressure is weak, but $\mathrm{pH}$ and salinity have larger impacts [17]. In the Arctic environment the salinity near the surface is much lower than the normal 35 parts per thousand found throughout much of the oceans [18]. As a result, less attenuation occurs than in open oceans.

As a general rule of thumb, there is a $10 \mathrm{~dB}$ drop in sound intensity as follows:

- $100 \mathrm{~Hz} r_{-10 d B} \approx 2200 \mathrm{~km}$

- $1 \mathrm{kHz} r_{-10 d B} \approx 145 \mathrm{~km}$

- $10 \mathrm{kHz} r_{-10 d B} \approx 9 k m$

Although sound attenuates rapidly as frequency increases, it still propagates much further in water than electromagnetic waves, which are typically absorbed within a few meters [9].

\subsubsection{Acoustic Reciprocity}

This is the theory that between any particular source and receiver, when swapped, the same pressure field is present in the reverse scenario. This means for linear acoustics, when evaluating a ray diagram (eg. Figure 1-3), stopping at any point along one of the ray paths, and placing the source at that location, the same exact ray path exists heading back toward 


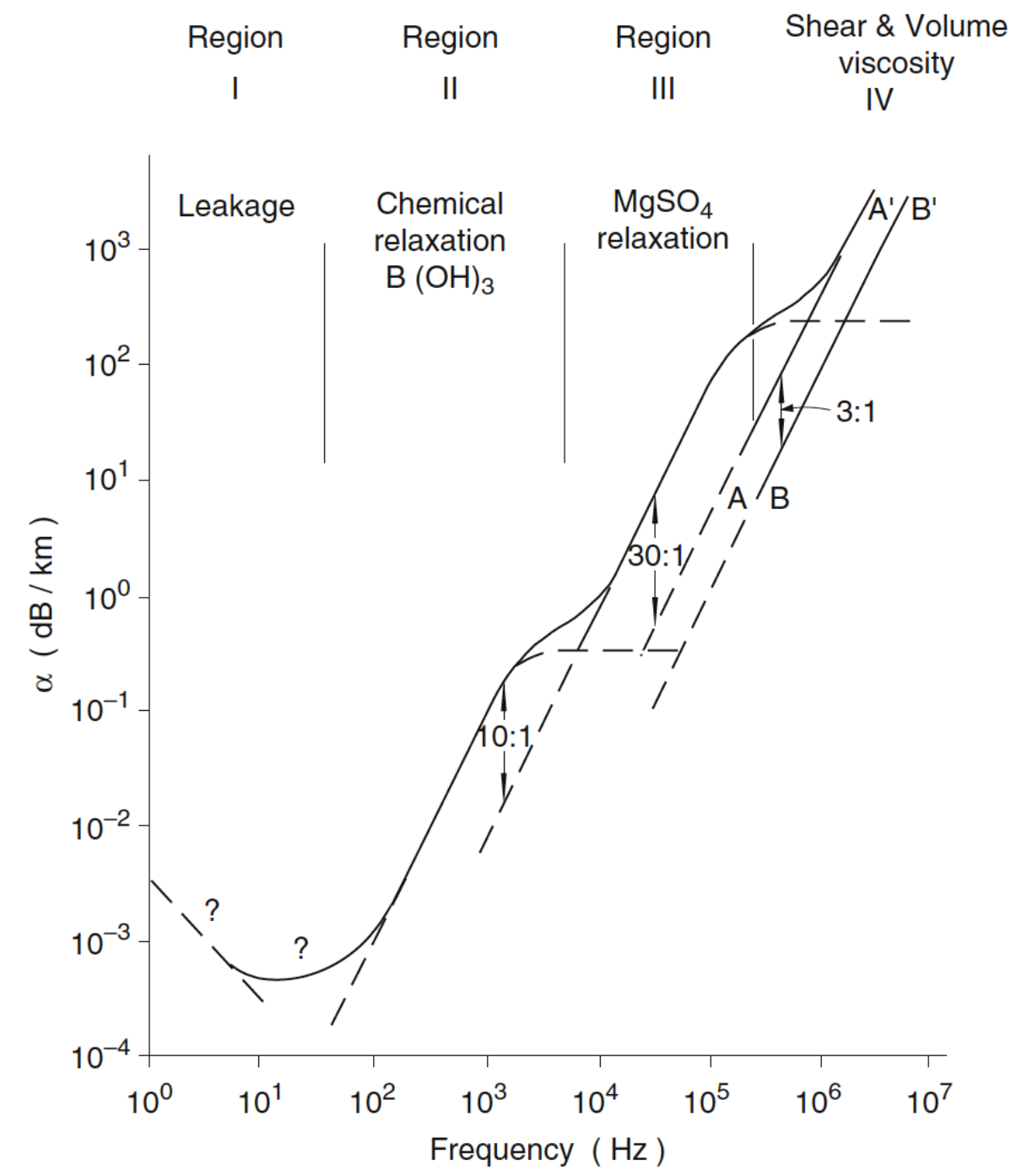

Figure 2-3: Regions of different dominant processes of attenuation of sound in sea water. [16]

the original source location. This process certainly relies on the ocean acoustic environment to remain static. It assumes the SSP is not changing spatially or temporally.

As seen in Figure 2-4, the two submarines inside of the same environment with the same source levels, and the same receivers can hear one another the same. Moreover, the sound travels the same path between the two submarines as well, regardless of what that path may actually be. In my analysis of a ray trace diagram, when a propagation path potentially exists between one of the WHOI micro-modem buoys and Macrura, that same path also exists the other direction. 

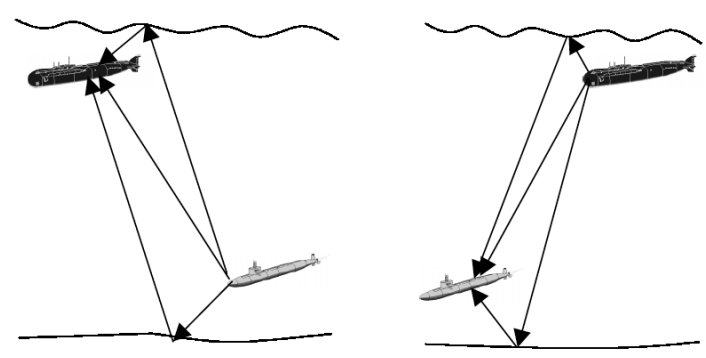

Figure 1-9. Acoustic Reciprocity (Homogenous Ocean).

Figure 2-4: Acoustic reciprocity between two submarines [19]
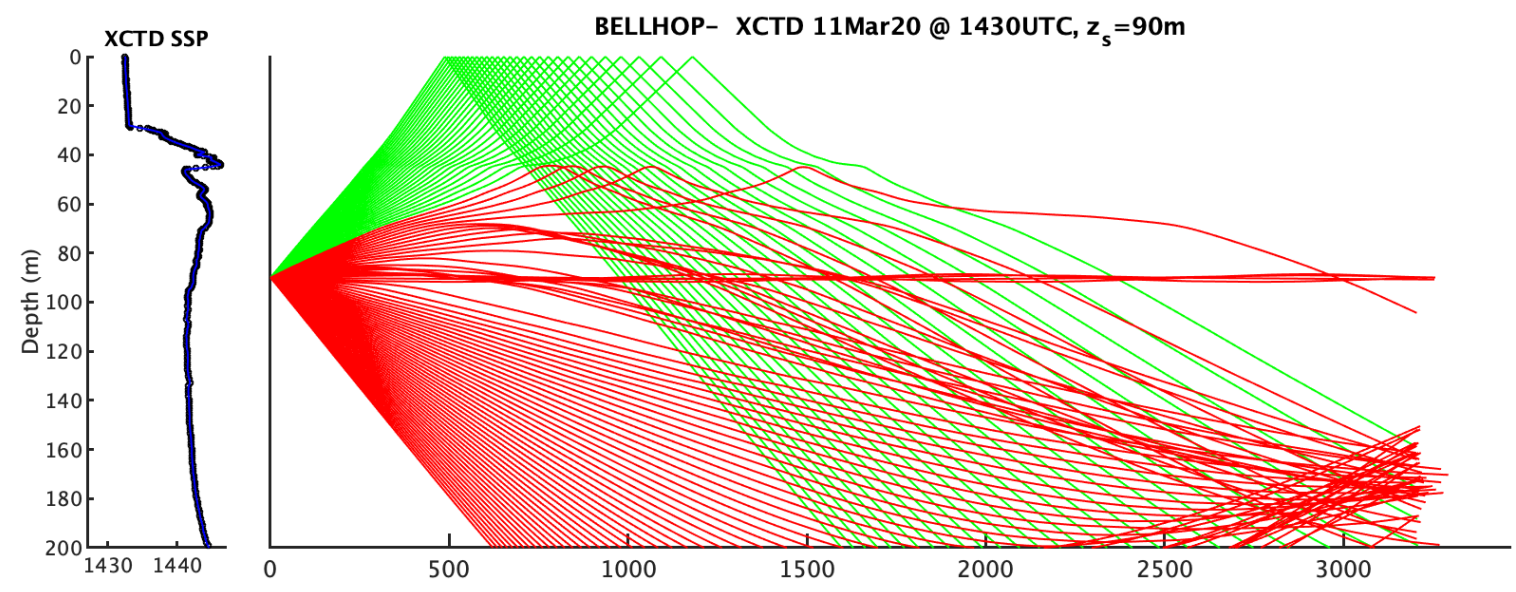

Figure 2-5: BELLHOP example for the 1430UTC XCTD with $z_{s}=90 \mathrm{~m}$. Rays must be defined

\subsubsection{BELLHOP}

BELLHOP is a beam tracing model that applies ray tracing, transmission loss, and other techniques to model acoustic waves throughout different ocean environments. It's a practical tool, and further descriptions can be found in The BELLHOP Manual and User's Guide [20]. I'll focused my analysis on the ray tracing portions of BELLHOP since I wanted to determine the reliable acoustic path the ray took to arrive at Macrura or the DURIP towed array. Figure 2-5 shows the BELLHOP analysis for ray tracing based on the XCTD taken at 1430UTC. This was the XCTD taken prior to the Vertical Run at 1655-1830UTC. When BELLHOP is used, a source depth must be defined, $z_{s}$. Here $z_{s}$ is set to $90 \mathrm{~m}$ based on the depth of buoy H1's transducer. The number of rays analyzed and spread of launch angles must also be defined. Here I'm using a $\pm 10^{\circ}$ launch angle with 101 rays. Effectively each ray represents $0.2^{\circ}$. This ray trace shows the propagation paths available based on the SSP at the time. 


\subsection{Digital Signal Processing}

\subsubsection{Aliasing}

Aliasing or folding occurs in digital signal processing when signals above the Nyquist Frequency are not filtered out of digitally sampled data, and these signals present themselves at lower frequencies than their original frequencies. Typically a filter is applied to prevent aliasing from occurring, but without a filter, aliasing occurs naturally. The Nyquist frequency is $f_{\text {Nyquist }}=1 / 2 * f_{s}$ where $f_{s}$ is the sampling frequency. A simple example of aliasing is shown in Figure 2-6. Three separate functions are compared: $x_{1}=\cos (0.4 \pi t)$, $x_{2}=\cos (2.4 \pi t)$, and $x_{3}=\cos (1.6 \pi t)$. It's important to recall the relationship between the sampling frequency, sampling period, and the relationship between frequency in radians/sec or Hz:

$$
T_{s}=\frac{1}{f_{s}} \quad \text { and } \quad \omega=2 \pi f
$$

Applying a sampling frequency, $f_{s}$, of $1 \mathrm{~Hz}$ results in a sampling period of $T_{s}=1$ second. The Nyquist frequency, $f_{\text {Nyquist }}=0.5 \mathrm{~Hz}$. Frequencies above $0.5 \mathrm{~Hz}$ or with $\omega>\pi$ radians/sec are aliased. Since $\omega_{2}=2.4 \pi$ and $\omega_{3}=1.6 \pi$ are both larger than $\pi ; x_{2}$ and $x_{3}$ are aliased. Uniquely in this scenario, $x_{2}$ and $x_{3}$ are aliased directly into $x_{1}$. Figure 2-6 shows the three signals, and that with $T_{s}=1$ second, the samples are indistinguishable between the different signals. There are a few things to consider regarding aliasing.

First, it's impossible to distinguish any of these signals from one another once sampling is complete. So, if $x_{3}=\cos (1.6 \pi t)$ were the only real signal to exist while sampling took place, it would be lost and assumed to be $x_{1}=\cos (0.4 \pi t)$ instead. This means that when evaluating the frequency content of the signal after sampling at $f_{s}=1 \mathrm{~Hz}$, you would incorrectly determine there was a signal at $\omega_{1}=0.4 \pi$ or $f_{1}=\frac{\omega_{1}}{2 \pi}=0.2 \mathrm{~Hz}$. This signal at $0.2 \mathrm{~Hz}$ is aliasing at work. The original content was only $\omega_{3}=1.6 \pi$ or $f_{3}=\frac{\omega_{3}}{2 \pi}=0.8 \mathrm{~Hz}$ which is greater than the Nyquist frequency of $f_{\text {Nyquist }}=1 / 2 * f_{s}=0.5 \mathrm{~Hz}$. As a result, that content is at risk for aliasing.

Second, the Nyquist frequency plays a key role in determining the necessary sampling frequency for capturing a signal of interest. Another way to describe the Nyquist frequency is to consider the Shannon/Nyquist Sampling Theorem: A continuous-time signal $x(t)$ with frequencies no higher than $f_{\max }$ can be reconstructed exactly from its samples, if the samples 


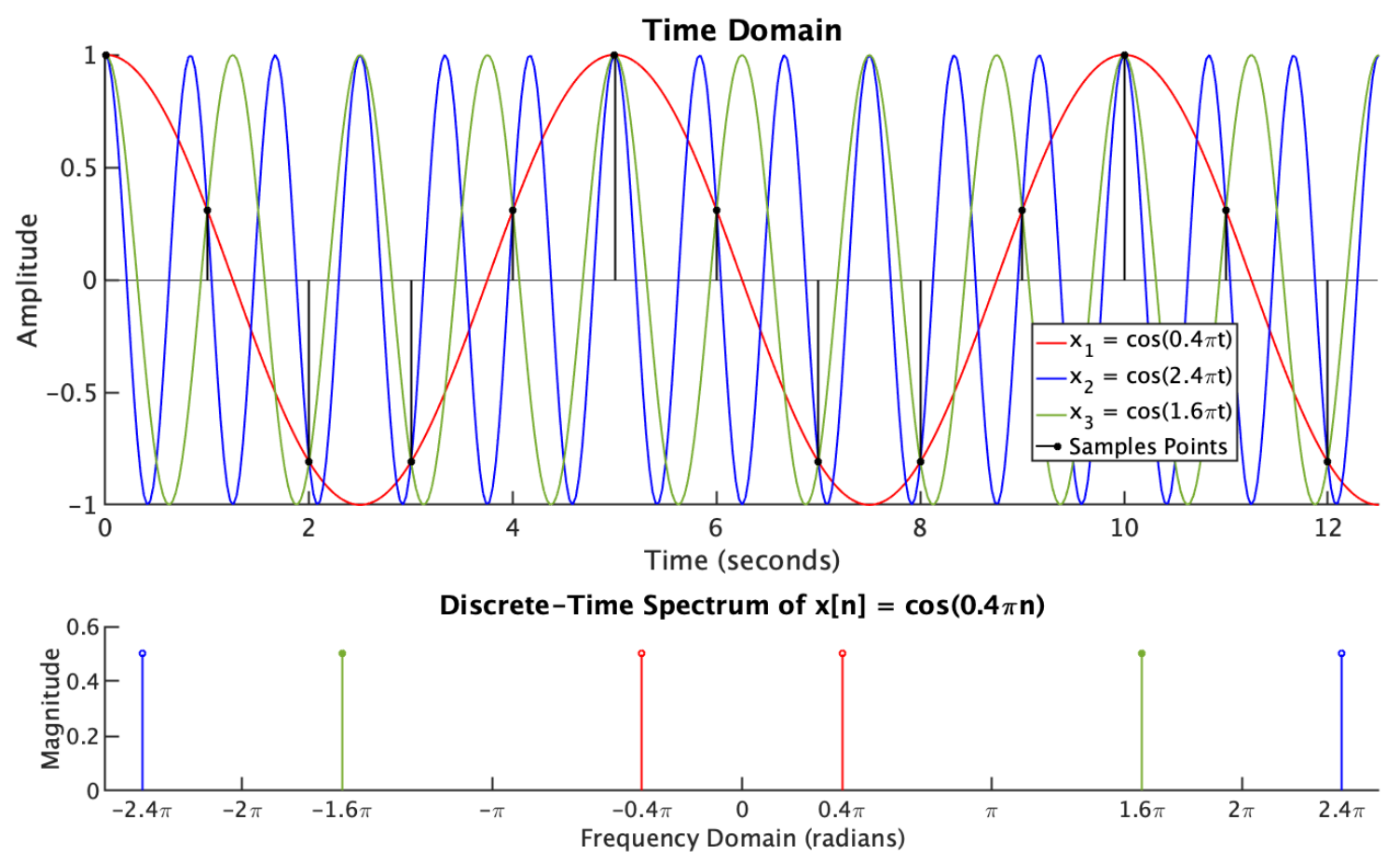

Figure 2-6: Aliasing for $x_{1}=\cos (0.4 \pi t)$. Although $x_{2}$ and $x_{3}$ are different functions, when $T_{s}=1$ second, the samples align with all three functions. As a result, they are said to be aliased. When looking at the frequency domain of the samples, it's clear that $x_{1}$ is effectively repeating itself every $\pm 2 \pi \mathrm{n}$ where $\mathrm{n}=0,1,2, \ldots$ In this context, it's impossible to distinguish $x_{1}, x_{2}$, and $x_{3}$ from one another without adjusting $T_{s}$.

are taken at a rate that is greater than $2 * f_{\max }[21]$.

Lastly, to prevent unnecessarily capturing signals above the Nyquist frequency which would result in aliasing, a low pass filter is typically applied with a cutoff frequency, $f_{c}$ near the Nyquist frequency. In the example above, if a filter were used to remove all signals above $0.5 \mathrm{~Hz}, f_{N y q u i s t}$, then $x_{3}$ wouldn't be present when the sampling took place, since $f_{3}>f_{c}$. As a result, although $x_{3}$ is actually present, it wouldn't be represented by our sampling, and it wouldn't be aliased down to $0.2 \mathrm{~Hz}$. If for some reason we wanted capture this signal, we could apply the Shannon/Nyquist Sampling Theorem, and adjust the sampling frequency to at least $1.6 \mathrm{~Hz}$. Since $f_{s} \geq 2 f_{\max }$, where $f_{\max }$ represents the maximum frequency you're attempting to capture and be able to accurately reproduce.

\section{ICEX 2020 Aliasing Example}

During ICEX we employed the DURIP towed array with a sampling frequency of $f_{s}=12$ $\mathrm{kHz}$. This means that the Nyquist frequency is $f_{\text {Nyquist }}=6 \mathrm{kHz}$, and $f_{\max }=6 \mathrm{kHz}$. If 
frequencies above $6 \mathrm{kHz}$ are captured, they can't accurately be reproduced because they are aliased down below $6 \mathrm{kHz}$. Aliasing would create the illusion of signals below $6 \mathrm{kHz}$ that weren't actually present.

For example, the WHOI micro-modem we employed used a $10 \mathrm{kHz}$ carrier frequency. A $10 \mathrm{kHz}$ signal was transmitted into the water every time the micro-modem was communicating. If this $10 \mathrm{kHz}$ signal was recorded by the DURIP towed array, it's aliased since it's above the $f_{\text {Nyquist }}$ of $6 \mathrm{kHz}$. Since the $10 \mathrm{kHz}$ signal is under-sampled, folding occurs and we want to know where to look for the aliased signal.

$$
\omega= \pm \frac{\omega_{o}}{f_{s}}+2 \pi \mathrm{n}= \pm \frac{2 \pi f_{o}}{f_{s}}+2 \pi \mathrm{n} \quad \text { with } \mathrm{n}=0, \pm 1, \pm 2, \ldots
$$

In our particular case for ICEX:

$$
\begin{gathered}
\omega=\frac{2 \pi(10 k H z)}{12 k H z}-2 \pi \mathrm{n} \text { and } \frac{-2 \pi(10 k H z)}{12 k H z}+2 \pi \mathrm{n} \\
\omega=\frac{5}{3} \pi-2 \pi=\frac{1}{3} \pi \text { and }-\frac{5}{3} \pi+2 \pi=-\frac{1}{3} \pi \\
\omega=\frac{1}{3} \pi=\frac{2 \pi f_{\text {aliased }}}{f_{s}} \Rightarrow f_{\text {aliased }}=\frac{1}{3} * \frac{12}{2}=2 k H z
\end{gathered}
$$

Therefore the $10 \mathrm{kHz}$ signal transmitted by the WHOI micro-modem aliases or folds down to $2 \mathrm{kHz}$ on the DURIP towed array. This means that when evaluating data on the

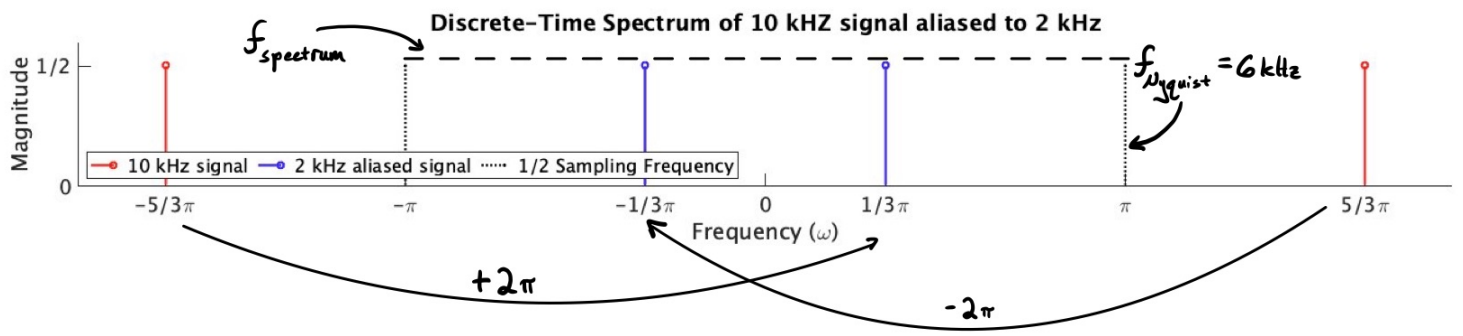

Figure 2-7: During ICEX $f_{s}=12 \mathrm{kHz}$ for the DURIP towed array. The $10 \mathrm{kHz}$ transmissions from the WHOI micro-modem are under-sampled and alias down to $2 \mathrm{kHz}$.

DURIP towed array, any spurious data around $2 \mathrm{kHz}$ on a spectrogram could potentially be aliased from the $10 \mathrm{kHz}$ micro-modem transmissions, or other $10 \mathrm{kHz}$ noise present in the water. Figure 2-7 shows this folding that occurs with the $10 \mathrm{kHz}$ signal from the micromodem and how it would interfere with $2 \mathrm{kHz}$ signals that are actually present in the water. Figure 2-8 shows the same problem from the time domain perspective. It's clear that both 


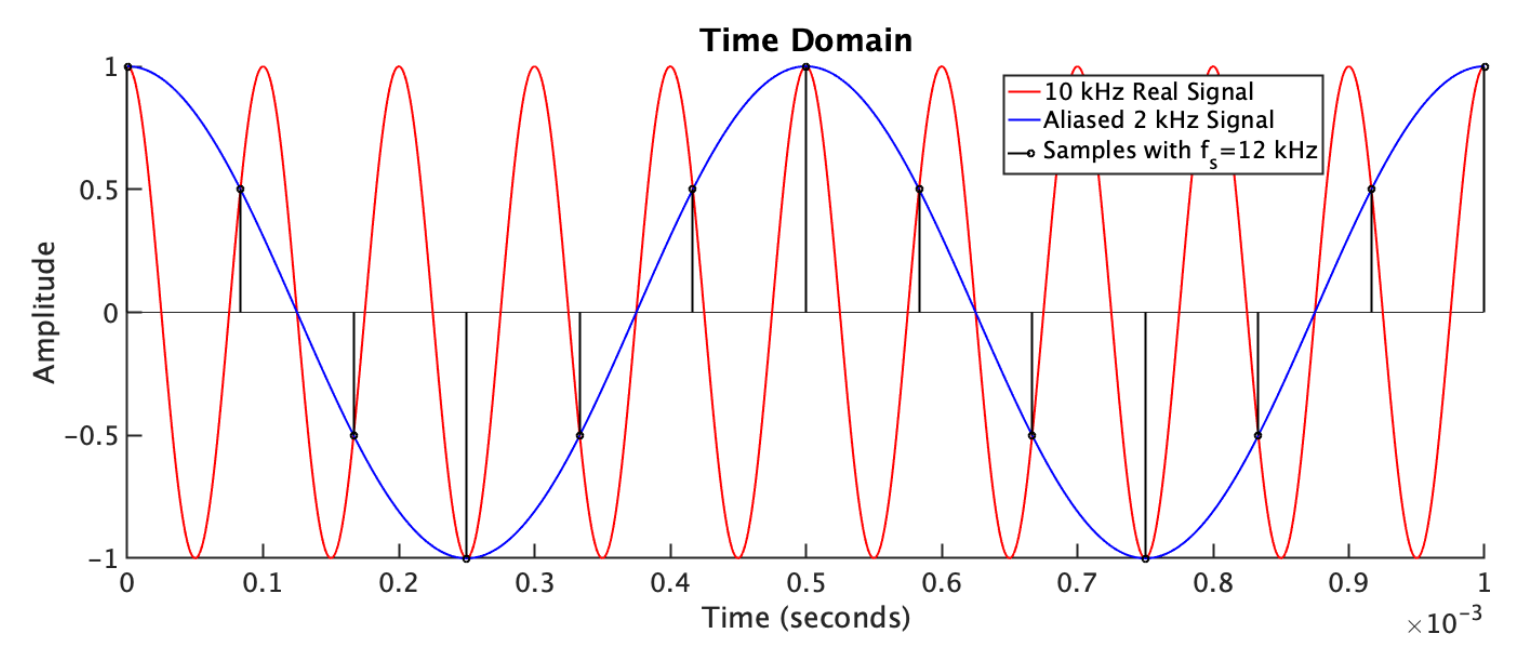

Figure 2-8: $f_{s}=12 \mathrm{kHz}$. A $10 \mathrm{kHz}$ and $2 \mathrm{kHz}$ signal are indistinguishable.

$2 \mathrm{kHz}$ and $10 \mathrm{kHz}$ signals are sampled at the same points and would interfere with one another for a sampling frequency of $f_{s}=12 \mathrm{kHz}$. In the case of the DURIP towed array, this isn't a problem due to the low pass filter in the DAS. The spectrogram in Figure 1-10 doesn't show a signal around $2 \mathrm{kHz}$, showing how effective the low pass filter is at removing the high frequency content we don't want to alias.

\subsection{Beamforming}

The concept of beamforming ties together elements of digital signal processing, and the fundamentals of ocean acoustics. Combining signal processing with the wave equation enables beamforming. In order to beamform we need an array or a series of hydrophones that work in unison. This discussion focuses on linear arrays, similar to the DURIP towed array, see Figure 1-8.

Beamforming enables an array to determine the direction of arrival of a signal. I focused on conventional beamforming, and since the array is linear the beams do not include fidelity in $\phi$. Figure 2-9a shows the spherical coordinate system with $z$ representing the depth axis along which the array is located. Throughout the Vertical Run, the DURIP towed array was effectively a vertical array in line with the z-axis as well. So, $\theta$ represents the Depression Elevation $(\mathrm{D} / \mathrm{E})$, and $\phi$ cannot be determined. 


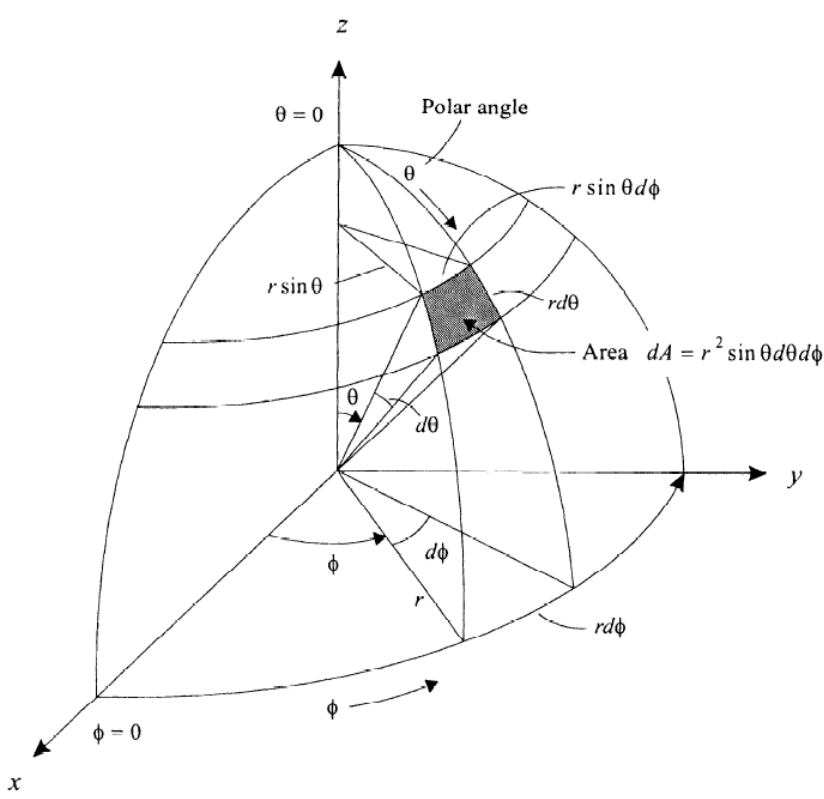

(a) Spherical coordinate system.

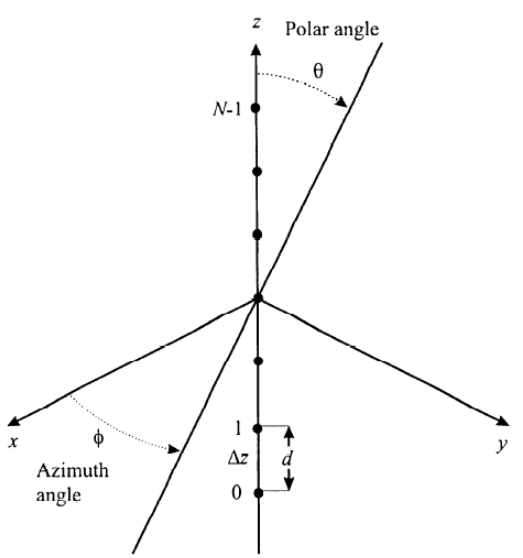

(b) The elements of the linear array are along the z-axis. The polar angle, $\theta$, is also referred to as the Depression Elevation $(\mathrm{D} / \mathrm{E})$. The azimuthal angle is indeterminate.

Figure 2-9: Coordinate system and linear array conventional element locations. [14]

\subsubsection{Beamforming Fundamentals for Linear Arrays}

Mathematically we describe the wavenumber $\mathbf{k}$ as:

$$
\mathbf{k}=-\frac{2 \pi}{\lambda}\left[\begin{array}{c}
\sin (\theta) \cos (\phi) \\
\sin (\theta) \sin (\phi) \\
\cos (\theta)
\end{array}\right]=-\frac{2 \pi}{\lambda} \mathbf{u} \quad \text { where } \quad \mathbf{u}=\left[\begin{array}{c}
u_{x} \\
u_{y} \\
u_{z}
\end{array}\right]
$$

The linear array is described by a series of $\mathrm{N}$ elements at positions $\mathbf{p}_{n}: n=0,1,2, \ldots, N-1$ along the z-axis as shown in Figure 2-9b. This is effectively the same as the DURIP towed array while it's drooping during the Vertical Run.

Sampling each of these N-elements results in vector $\mathbf{f}(t, \mathbf{p})$. Each element is evaluated by a linear time-invariant (LTI) filter with a given impulse response $h_{n}(\tau)$. In order to obtain the array output, $y(t)$ we perform a convolution of the impulse response and the signal:

$y(t)=\sum_{n=0}^{N-1} \int_{-\infty}^{\infty} h_{n}(t-\tau) f_{n}\left(\tau, \mathbf{p}_{n}\right) d \tau \quad$ or in vector notation $\quad y(t)=\int_{-\infty}^{\infty} \mathbf{h}^{T}(t-\tau) \mathbf{f}(\tau, \mathbf{p}) d \tau$

We can take the Fourier transform of $y(t)$, our array output, and view it in the frequency 
domain, $\omega$ instead of the time domain, $t$.

$$
Y(\omega)=\int_{-\infty}^{\infty} y(t) e^{-j \omega t} d t=\mathbf{H}^{T}(\omega) \mathbf{F}(\omega)
$$

This leads to the array manifold vector:

$$
\mathbf{v}_{\mathbf{k}}(\mathbf{k})=\left[\begin{array}{c}
e^{-j \mathbf{k}^{T} \mathbf{p}_{0}} \\
e^{-j \mathbf{k}^{T} \mathbf{p}_{1}} \\
\vdots \\
e^{-j \mathbf{k}^{T} \mathbf{p}_{N-1}}
\end{array}\right]
$$

This allows us to describe $f$ and $F$, it's Fourier Transform pair, as follows:

$$
\mathbf{f}(t, \mathbf{p})=e^{j \omega t} \mathbf{v}_{\mathbf{k}}(\mathbf{k}) \quad \Rightarrow \quad \mathbf{F}(\omega)=F(\omega) \mathbf{v}_{\mathbf{k}}(\mathbf{k})
$$

As a result, we can also describe $y$ and $Y$ as follows:

$$
y(t, \mathbf{k})=\mathbf{H}^{T}(\omega) \mathbf{v}_{\mathbf{k}}(\mathbf{k}) e^{j \omega t} \quad \Rightarrow \quad Y(\omega, \mathbf{k})=\mathbf{H}^{T}(\omega) \mathbf{v}_{\mathbf{k}}(\mathbf{k})
$$

In order to adjust the gain and phase at the output we apply complex weights, $w_{n}^{*}$. These complex weights, $w_{n}^{*}$, take the place of the impulse response, $h_{n}(\tau)$, where the analysis is normalized. This leads to the complex weight vector:

$$
\mathbf{w}^{H}=\left[\begin{array}{c}
w_{0}^{*} \\
w_{1}^{*} \\
\vdots \\
w_{N-1}^{*}
\end{array}\right] \quad \text { or equivalently } \quad \mathbf{w}^{H}=\mathbf{H}^{T}\left(\omega_{c}\right) \text { where } \omega_{c} \text { is the carrier frequency. }
$$

and subsequently we arrive at $y$ :

$$
y(t, \mathbf{k})=\mathbf{w}^{H} \mathbf{v}_{\mathbf{k}}(\mathbf{k}) e^{j \omega t} \Rightarrow Y(\omega, \mathbf{k})=\mathbf{w}^{H} \mathbf{v}_{\mathbf{k}}(\mathbf{k})
$$

I'm focusing my discussion on two particular spaces. Either between $0 \leq \theta \leq \pi$ or between $-1 \leq u_{z} \leq 1$. Recall $u_{z}=\cos (\theta)$, and although $u_{z}$ is defined between $-\infty$ to $\infty$, it's the visible region is between -1 and 1 . Typically $d \leq \lambda / 2$ for a standard linear array. 
If we allow $d>\lambda / 2$ then $\mathrm{u}$-space is helpful to understand what to anticipate. In $\mathrm{u}$-space what's happening between -1 to 1 is effectively repeating outside of those bounds. When $d>\lambda / 2$ we effectively squeeze more of what's happening outside of -1 to 1 inside the visible window of $-1 \leq u \leq 1$.

In frequency space, we refer to the array output as the beam pattern. The beam pattern in $\theta$ space is:

$$
B_{\theta}(\theta)=\mathbf{w}^{H} \mathbf{v}_{\theta}(\theta)=e^{-j\left(\frac{N-1}{2}\right) \frac{2 \pi d}{\lambda} \cos (\theta)} \sum_{n=0}^{N-1} \omega^{*} e^{j n \frac{2 \pi d}{\lambda} \cos (\theta)}, \quad 0 \leq \theta \leq \pi
$$

and the beam pattern in u-space is:

$$
B_{u}(u)=\mathbf{w}^{H} \mathbf{v}_{u}(u)=e^{-j\left(\frac{N-1}{2}\right) \frac{2 \pi d}{\lambda} u} \sum_{n=0}^{N-1} \omega^{*} e^{j n \frac{2 \pi d}{\lambda} u}, \quad-1 \leq u \leq 1 .
$$

If the array is uniformly weighted then $w_{n}=\frac{1}{N}, n=0,1,2, \ldots, N-1$, and as a result the beam pattern is:

$$
\begin{gathered}
B_{\theta}=\frac{1}{N} \frac{\sin \left(\frac{N}{2} \frac{2 \pi}{\lambda} \cos (\theta) d\right)}{\sin \left(\frac{1}{2} \frac{2 \pi}{\lambda} \cos (\theta) d\right)}, \quad 0 \leq \theta \leq \pi \\
B_{u}=\frac{1}{N} \frac{\sin \left(\frac{\pi N d}{\lambda} u\right)}{\sin \left(\frac{\pi d}{\lambda} u\right)}, \quad-1 \leq u \leq 1
\end{gathered}
$$

Recall that $d=\Delta z$ or the spacing between elements on the array.

\subsubsection{Plotting Beam Patterns}

Plotting the beam pattern and comparing them in Figure 2-10 shows the difference between polar coordinates and u-space. The beam patterns are plotted on a dB scale. This is how the plots in Figure 2-10 are generated. The dB levels are calculated:

$$
Y_{d B}(\omega)=10 \log _{10}\left(|Y(\omega)|^{2}\right)
$$

In polar coordinates and $\mathrm{u}$-space this translates to:

$$
Y_{d B}(\theta)=20 \log _{10}\left(B_{\theta}(\theta)\right) \quad \text { or } \quad Y_{d B}(u)=20 \log _{10}\left(B_{u}(u)\right)
$$

Figure 2-10 also shows the different lobes discussed with beamforming. The main lobe is 


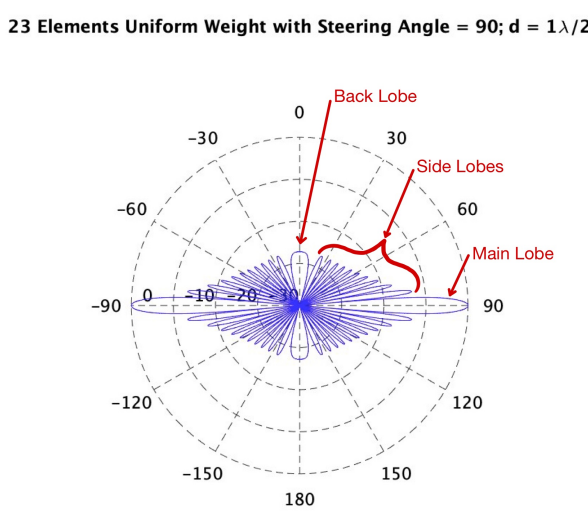

(a) Polar plot of beam pattern for a 23 element linear array

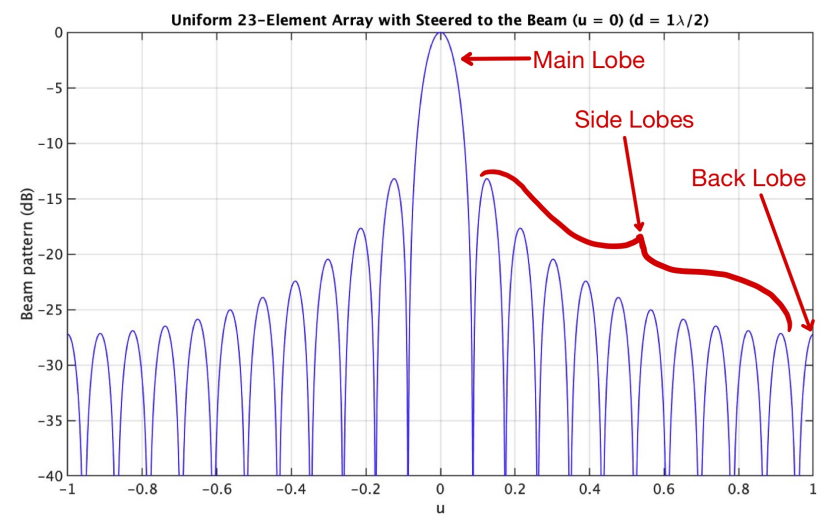

(b) Plot of beam pattern for a 23 element linear array in u-space.

Figure 2-10: Polar plot and u-space plot of beam pattern for a 23 element linear array with $d=\frac{\lambda}{2}$.

the direction where the the array is focused. The side lobes are important when beamforming because anything in a side lobe could potentially show up at the "wrong" bearing if there isn't a sufficient $\mathrm{dB}$ drop. The back lobe is the lobe that when $d=\frac{\lambda}{2}$ is $90^{\circ}$ off from the main lobe (ie. when steering the array to the beam, the lobe present in FWD or AFT endfire. In Figure 2-10a the array is steering at $90^{\circ}$ relative, and FWD and AFT endfire are bearing $0^{\circ}$ and $180^{\circ}$ respectively).

Generally the polar plot is more intuitive. In Figure 2-10a, the z-axis is modeled by the vertical line from $0^{\circ}$ to $180^{\circ}$. This is where the elements of the array are located, and the array is presently steered to the beam or $90^{\circ}$ relative. In the vertical DURIP towed array configuration, this would be akin to sound traveling straight from a source to the vertical array, similar to Figure 2-1 if the ear represented the vertical array and no sound wave bending took place. It's important to recall that in this configuration, the azimuthal angle is indeterminate. So steering to an angle of $+45^{\circ}$ is equivalent to steering to an angle of $-45^{\circ}$.

\subsubsection{Grating Lobes}

U-space is not as intuitive initially, but it provides additional insight into the behaviour of beamforming and its dependence on the wavenumber or element spacing. It helps you understand what happens when $d>\frac{\lambda}{2}$. Figure 2-11a shows how the the u-space plot is helpful since it looks outside the visible region. The the polar plot is limited to the visible 


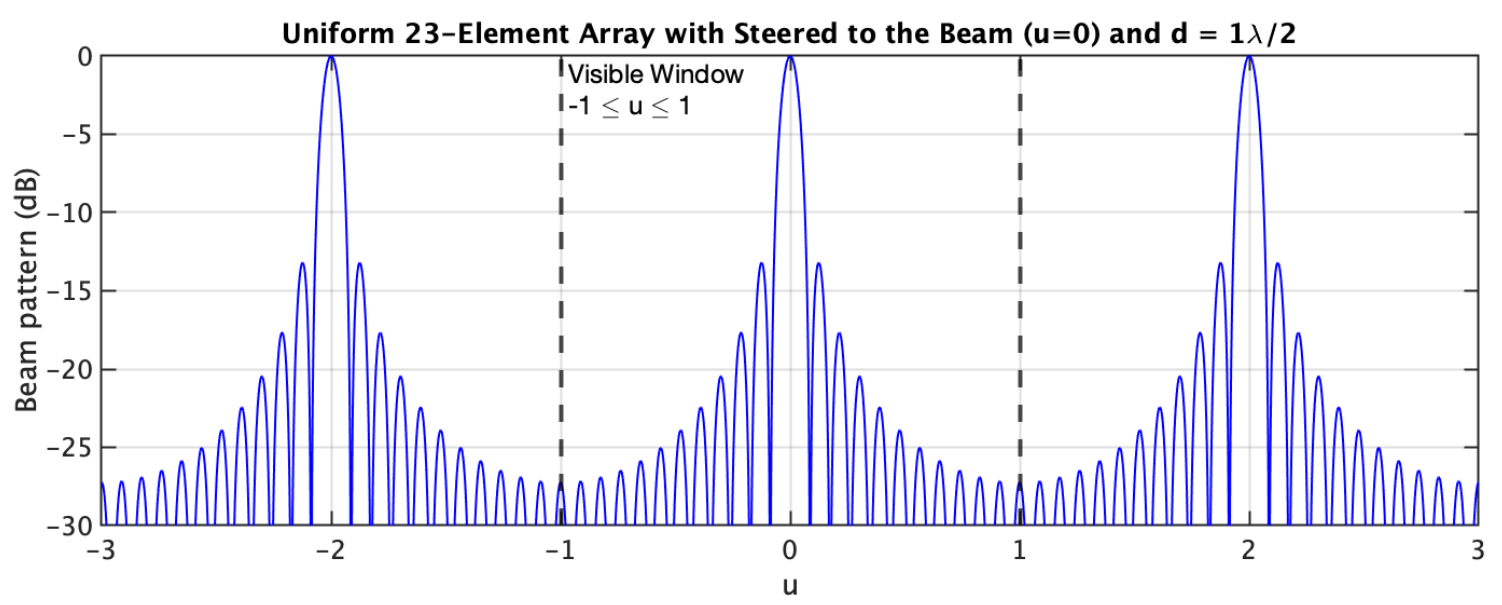

(a) u-space when $d=\frac{\lambda}{2}$ showing outside the visible region.

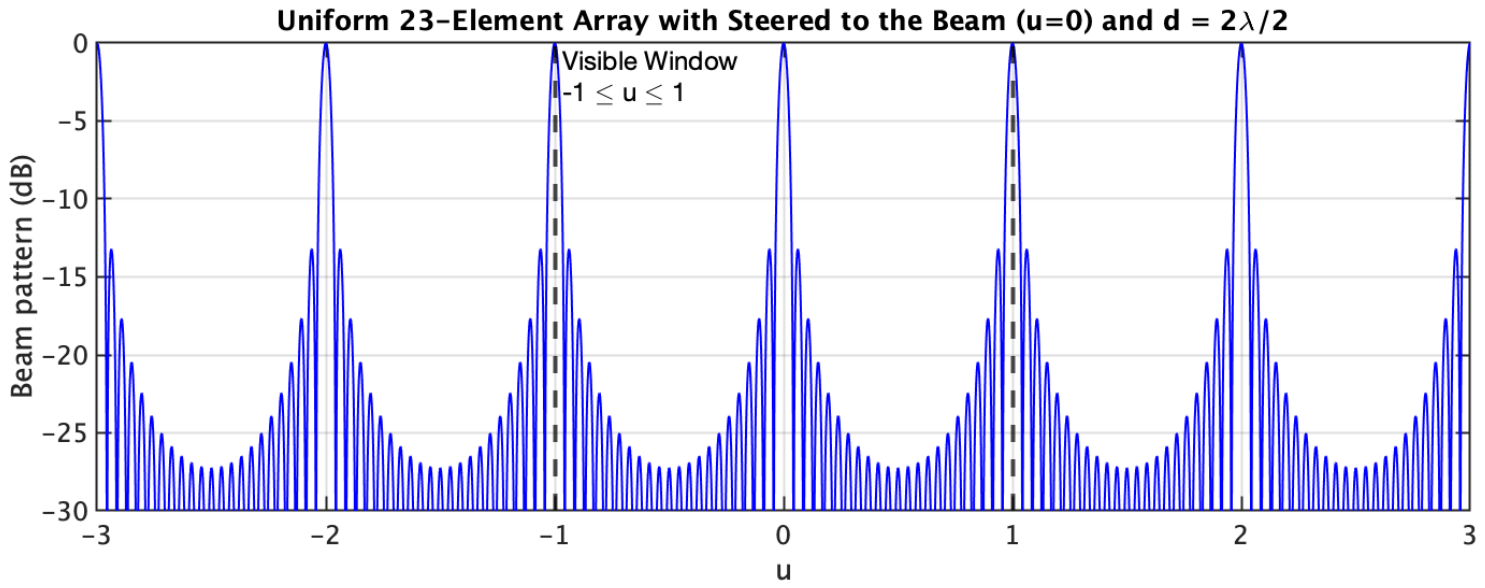

(b) u-space when $d=\lambda$. Similar to Figure 2-11a, just squeezed between -2 and 2 into the visible window, $-1 \leq u \leq 1$.

Figure 2-11: u-space visible region when $d=\lambda$ vice $d=\frac{\lambda}{2}$.

region, $0 \leq \theta \leq \pi$. When we allow $d>\frac{\lambda}{2}$, there is a compression of what's initially outside the visible region. The function gets compressed into the visible region of the beam pattern. Looking at the polar plot, it's hard to anticipate what to expect as you change the $f$ or $d$, but it's much easier to visualize this in u-space. Figure 2-11b shows the difference in the visible region when $d=\lambda$. This particular case also shows the grating lobes, which are identical to the main lobe. Anytime $d>\frac{\lambda}{2}$, grating lobes are a concern and begin to play a role in beamforming. Figure 2-12 shows the same grating lobes in a polar plot.

Appendix B shows additional beamformed polar plots and u-space plots for a 23 element linear array when $d>\frac{\lambda}{2}$. These plots help visualize the beam patterns in these cases. 


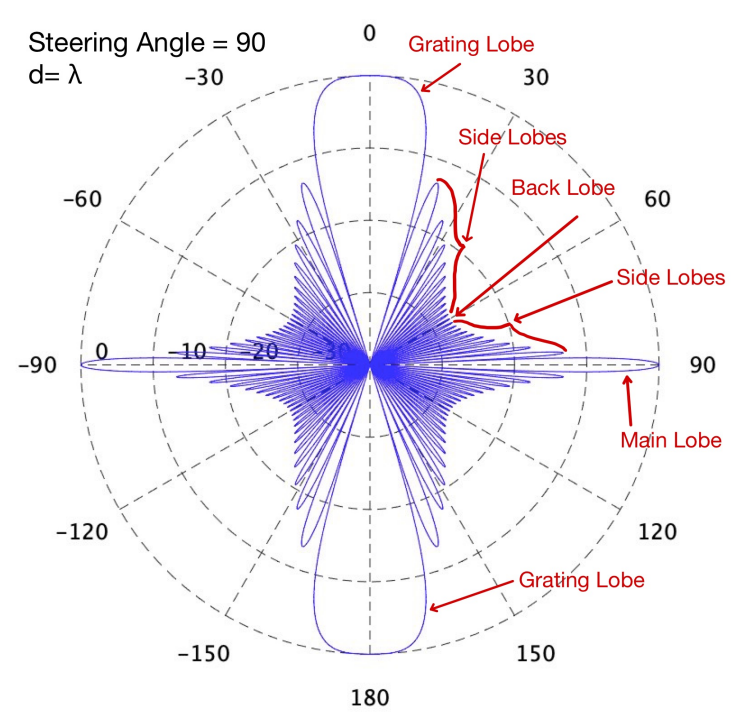

Figure 2-12: Polar plot with $d=\lambda$. Same as Figure 2-11b.

\section{Grating Lobes and Aliasing}

Note the similarity between $d \leq \lambda / 2$ and the equation for the Nyquist frequency from Chapter 1: $f_{\text {Nyquist }}=1 / 2 * f_{s}$. They seem similar because they are related. The spacing between nodes, $d \mathrm{~m}$, is similar to the time between samples (ie. $T_{\text {Nyquist }}=\frac{1}{f_{\text {Nyquist }}}$. If we converted the time between samples to a distance (eg. $T_{\text {Nyquist }} * c_{\text {air }}=\lambda_{\text {Nyquist }}$. This $\lambda_{\text {Nyquist }}$ is what we're effectively discussing in beamforming $d \leq \frac{\lambda}{2} \approx \lambda_{\text {Nyquist }}$.

The grating lobes in the beam patterns are aliasing. Grating lobes are just spatial aliasing vice temporal aliasing. Our earlier discussion was regarding temporal aliasing of frequencies above the $f_{\text {Nyquist }}$ that are potentially present in the frequency domain (recall we use a filter to prevent higher frequency content from aliasing down to a lower frequency). Here, grating lobes cause spatial aliasing which potentially results in sound showing up at the wrong angles. If we look at frequencies above the array $f_{c u t}$ (effectively the Nyquist frequency), grating lobes or aliasing occurs.

\subsubsection{Steering the Beams}

So far we've only looked at beam patterns on the beam of the array, $90^{\circ}$ relative, or normal to the array axis. However, in order to build a full plot of the acoustic information we need to steer the beams of the array throughout the entire viewing window (ie. $-1 \leq u \leq 1$ or $0 \leq \theta \leq \pi)$. This allows us to distinguish between acoustic sources at different arrival angles 
(eg. $60^{\circ}$ or $\left.135^{\circ}\right)$.

Mathematically we want $\mathbf{k}=\mathbf{k}_{T}$. $\mathbf{k}_{T}$ is the steering direction or main response axis. This steering direction adjusts the element function as follows:

$$
f_{s}(t, \mathbf{p})=e^{j \omega t} \mathbf{v}_{\mathbf{k}}\left(\mathbf{k}-\mathbf{k}_{T}\right) \quad \text { and array response is } \quad Y\left(\omega, \mathbf{k} \mid \mathbf{k}_{T}\right)=Y\left(\omega, \mathbf{k}-\mathbf{k}_{T}\right)
$$

Instead of two steps, we can apply the steering shift in conjunction with the weighting:

$$
\mathbf{w}=\frac{1}{N} \mathbf{v}_{\mathbf{k}}\left(\mathbf{k}_{T}\right) \quad \text { results in } \quad B_{c}\left(\mathbf{k}: \mathbf{k}_{T}\right)=\frac{1}{N} \mathbf{v}_{\mathbf{k}}^{H}\left(\mathbf{k}_{T}\right) \mathbf{v}_{\mathbf{k}}(\mathbf{k})
$$

$B_{c}$ is the conventional beam pattern for a linear array. This is used for beamforming on the DURIP towed array as well. This translates into polar and u-space as:

$$
\begin{gathered}
B_{\theta c}\left(\theta: \theta_{T}\right)=\frac{1}{N} \frac{\sin \left(\frac{\pi N d}{\lambda}\left(\cos (\theta)-\cos \left(\theta_{T}\right)\right)\right)}{\sin \left(\frac{\pi d}{\lambda}\left(\cos (\theta)-\cos \left(\theta_{T}\right)\right)\right)} \\
B_{u c}\left(u: u_{T}\right)=\frac{1}{N} \frac{\sin \left(\frac{\pi N d}{\lambda}\left(u-u_{T}\right)\right)}{\sin \left(\frac{\pi d}{\lambda}\left(u-u_{T}\right)\right)}
\end{gathered}
$$

Beamforming allows you to take the independent nodes and bring them together to build a full picture. Doing this no longer restricts the beam former to the beam ( $90^{\circ}$ relative) of the array. Now we're able to steer the beam former to any particular angle.

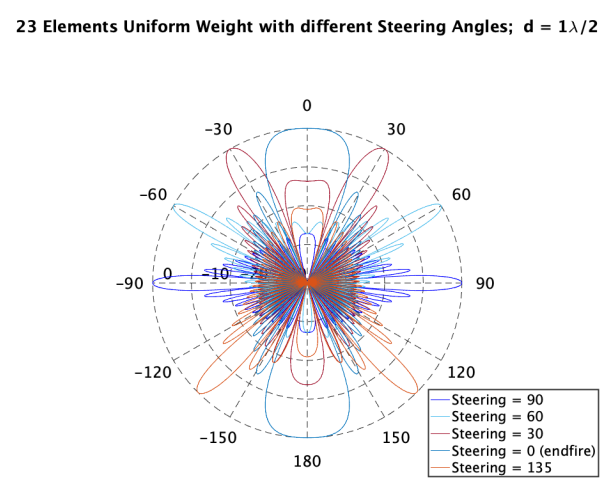

(a) Polar plot of beam pattern steered to endfire, $60^{\circ}, 30^{\circ}$, beam $\left(90^{\circ}\right)$, and $135^{\circ}$

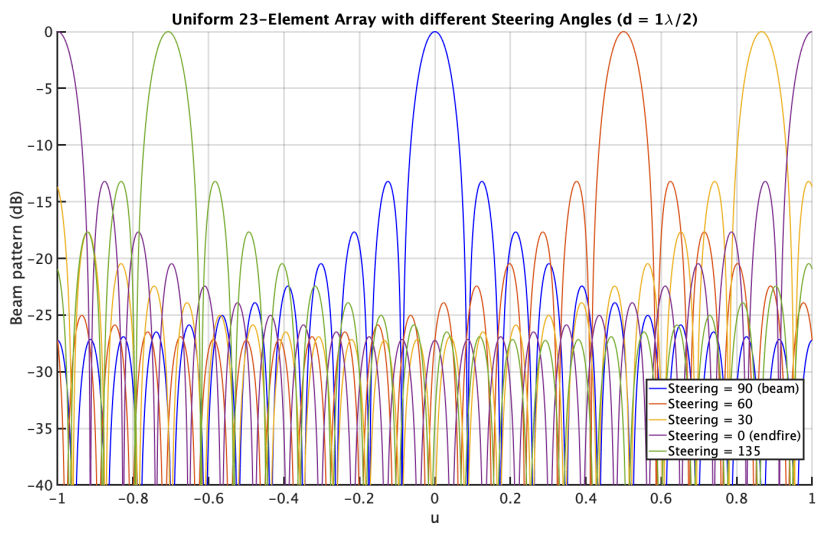

(b) Plot of beam pattern steered in u-space, endfire $\left(90^{\circ}\right), 60^{\circ}, 30^{\circ}$, the beam $\left(0^{\circ}\right)$, and $-45^{\circ}$.

Figure 2-13: Polar plot and u-space plot of beam patterns when array is steered to different angles. 
Figure 2-13 shows what the conventional beam pattern looks like when it is steered in polar coordinates and $\mathrm{u}$-space. The $\mathrm{u}$-space plot, Figure $2-13 \mathrm{~b}$, is somewhat deceptive due to the $u_{z}=\cos (\theta)$ dependence. So, the beam steering in u-space doesn't appear to result in any distortion to the main lobe. However, looking at the same plot in polar coordinates, Figure 2-13a, the distortion that occurs from steering the array is apparent. Clearly, when steering the array to $\mathrm{FWD}$ endfire $\left(0^{\circ}\right.$ relative $)$, the grating lobe is present at AFT endfire $\left(180^{\circ}\right.$ relative). The u-space plot shows the grating lobe's presence in aft endfire as well. However, it's unclear how substantial the beam width of the main lobe is when steered toward endfire from the u-space plot alone. Instead, the polar-plot shows this dependency more clearly.

The Half-Power Beamwidth (HPBW) is the point where there is a $-3 \mathrm{~dB}$ drop in the main lobe. This occurs when $\Delta u=H P B W=0.891 \frac{\lambda}{N d}$. This is specifically for the case when looking at the beam. In order for the HPBW to account for the steering direction we use the following equations:

$$
u_{R}=u_{T}+0.450 \frac{\lambda}{N d} \quad \text { and } \quad u_{L}=u_{T}-0.450 \frac{\lambda}{N d}
$$

Here $u_{R}$ and $u_{L}$ represent the right and left half-power points. In $\theta$ space this corresponds to:

$$
\cos \left(\theta_{R}\right)=\cos \left(\theta_{T}\right)-0.450 \frac{\lambda}{N d} \quad \text { and } \quad \cos \left(\theta_{L}\right)=\cos \left(\theta_{T}\right)+0.450 \frac{\lambda}{N d}
$$

In Figure 2-13 the plots are for a 23 element array with $d=\frac{\lambda}{2} \rightarrow \frac{\lambda}{d}=2$. This translates to a HPBW at FWD endfire, $\theta_{T}=0$ :

$$
\cos ^{-1}\left(\cos (0)-0.45 \frac{2}{23}\right)+\cos ^{-1}\left(\cos (0)+0.45 \frac{2}{23}\right)=2 \cos ^{-1}(1-0.039)=32.16^{\circ}
$$

Compared to the HPBW at the beam, $\theta_{T}=90$ :

$$
\cos ^{-1}\left(\cos (90)-0.45 \frac{2}{23}\right)+\cos ^{-1}\left(\cos (90)+0.45 \frac{2}{23}\right)=\cos ^{-1}(0-0.039)-\cos ^{-1}(0+0.039)=4.5^{\circ}
$$




\section{Chapter 3}

\section{ICEX 2020 Experiment}

The experiment for MIT-WHOI took place between 5-18 March 2020. The team arrived intermittently from various parts of the country and coalesced in Prudhoe Bay, AK, a small oil-town along the northern coast of Alaska on the Beaufort Sea. Here we joined members from Bluefin, and others from Arctic Submarine Lab (ASL) who coordinated sending each of us out to Ice Camp Seadragon, roughly 150 Nautical Miles (NM) Northeast of Prudhoe Bay. The entire team present on the ice is shown in Figure 3-1. Two critical players apart of professor Henrik Schmidt's group (LAMSS) at MIT were not able to make it onto the ice due to limited personnel restrictions. Their contributions were instrumental to the operation as a whole and merit acknowledgement as team members; Dr. Supun Randeni, and Dr. Eeshan Bhatt.

\subsection{Ice Camp Seadragon}

Flying into Ice Camp Seadragon from Prudhoe Bay is incredibly unique and described by those present, "like visiting another planet..." Unlike normal aviation operations, the pilot takes off after getting an updated GPS ice camp position from the ASL logistics-operations team in Prudhoe Bay. During the flight, the ice camp is moving with the ice and may be as far as a few NM from the original GPS location. Landing is complicated because the snow conditions make it extremely challenging for a pilot to maintain their depth perception, but instrument flight rules don't work because conditions on the ice are changing including the physical location and heading. ASL personnel spend a substantial amount of effort maintaining the runway as reasonably smooth as they can make it with the gear they've 


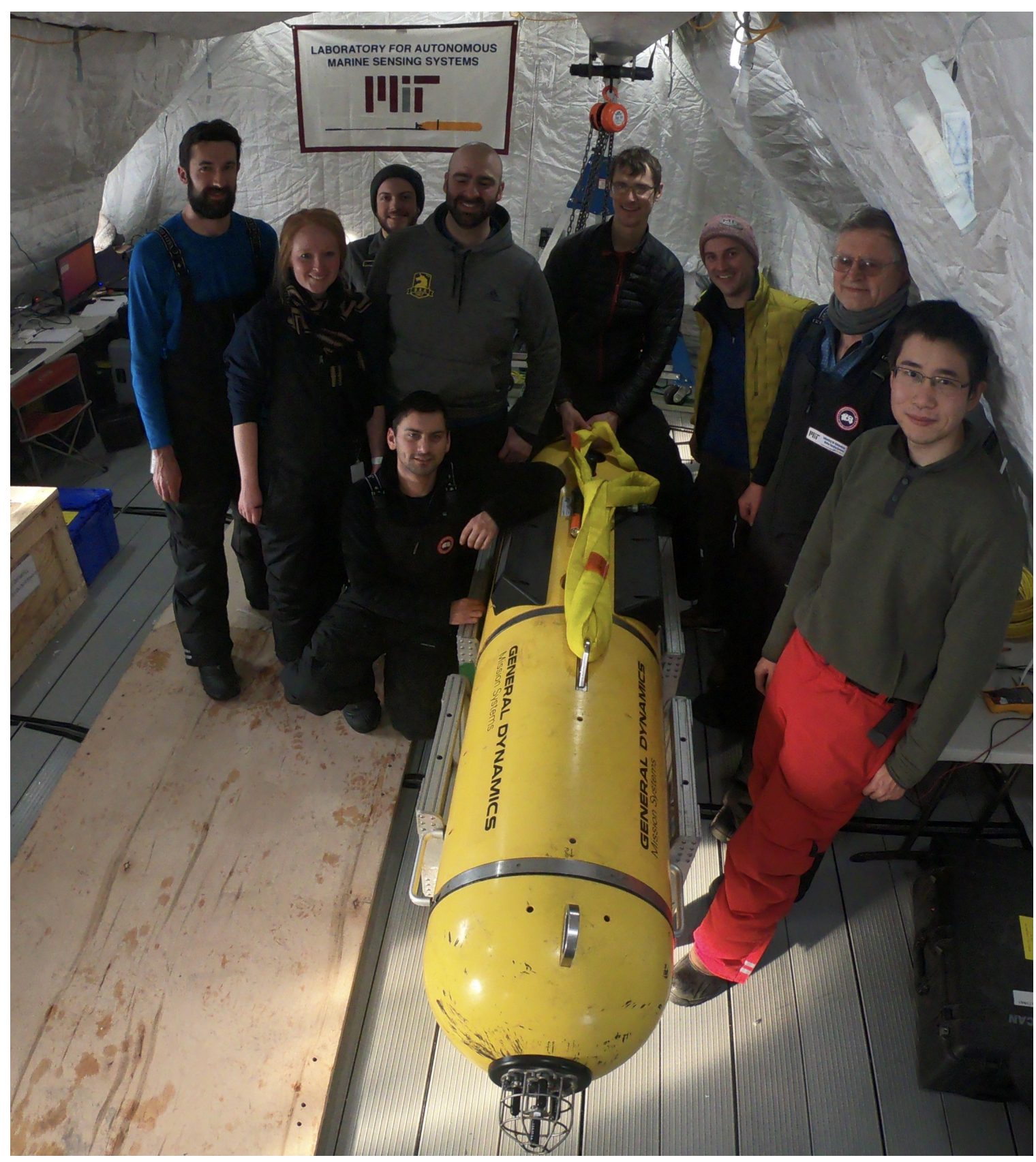

Figure 3-1: MIT-WHOI ICEX team inside the operations tent. Hydrohole is beneath the wooden planks on the floor. From left: Dan McDonald (Bluefin), Bradli Howard, Dennis Giaya (WHOI), Josiah Delange (Bluefin, kneeling), Oscar Viquez, Dr. Toby Schneider (GobySoft), Dan Goodwin, Dr. Henrik Schmidt, and Dr. Rui Chen.

got present on the ice to make it as safe as possible. Figure 3-2 shows a picture from the airplane coming into the ice camp. This shows the layout and provides a sense of the logistical planning necessary for this sort of evolution.

The hydrohole, Figure 3-3b, was topside in the operations tent, Figure 3-3a, where the 


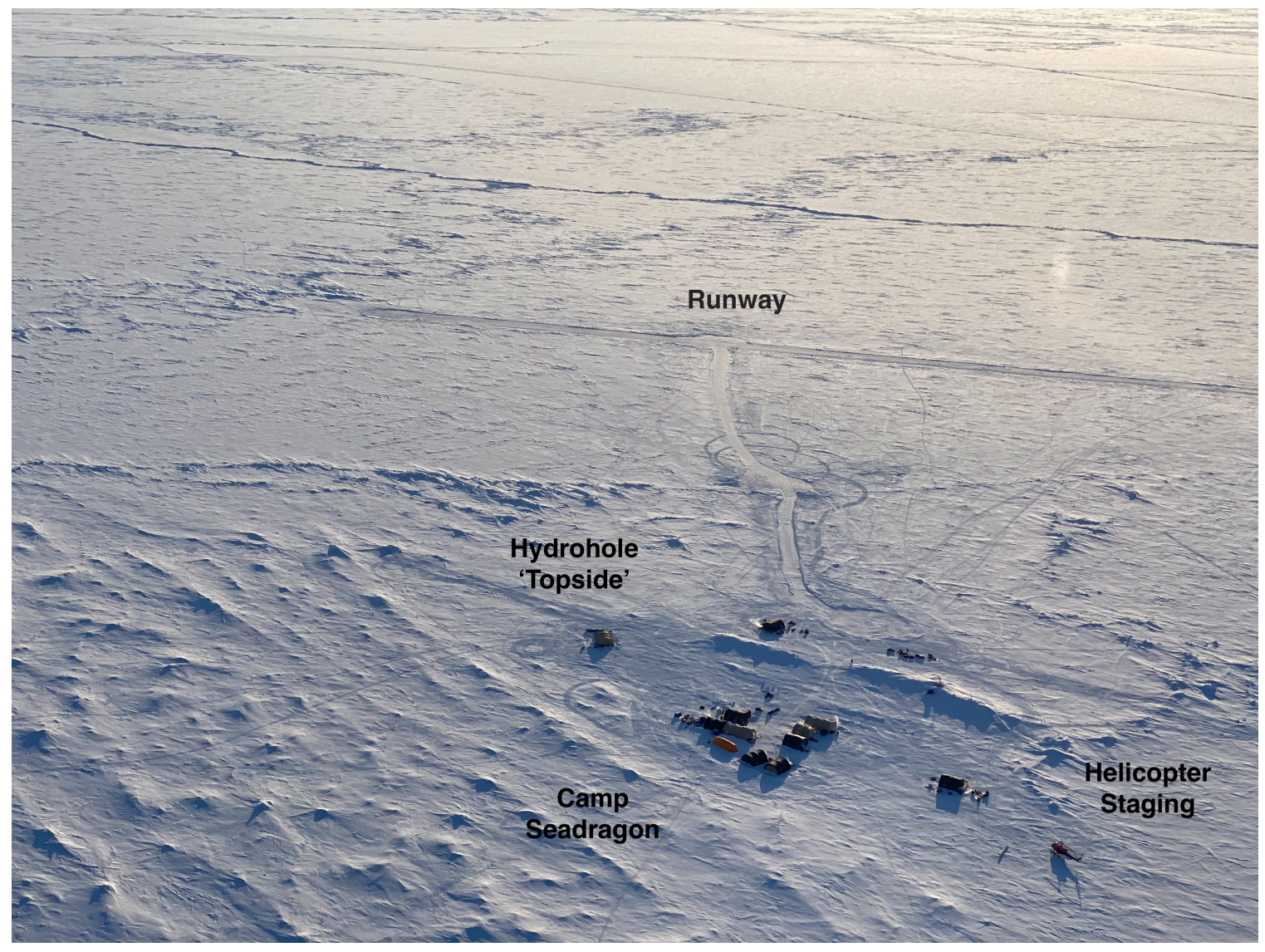

Figure 3-2: Aerial photograph while circling Ice Camp Seadragon to line-up with the runway.

MIT/WHOI team was located, and conducting their experiments from. A command tent was responsible for coordinating the operations between all parties present, including the submarines operating beneath the ice.

It's easy to lose sight of the political importance of this sort of event. Although, the Navy says ICEX is an, "opportunity for the U.S. Navy to assess its operational readiness in the Arctic and train with other services and partner nations and allies to increase experience in the region, and maintain regional stability while improving capabilities to operate in the Arctic environment" [22]. It's also signaling to other nations in the region that the U.S. presence in the Arctic is going to continue. While at Ice Camp Seadragon a fly over by Russian reconnaissance aircraft, TU-142s, occurred that was later reported in the news [23]. The TU-142s were flanked by U.S. fighter jets, F-22s and FA-18s, on either side. I took a picture of the event, Figure 3-4, and it serves as a small reminder that other countries are watching. 


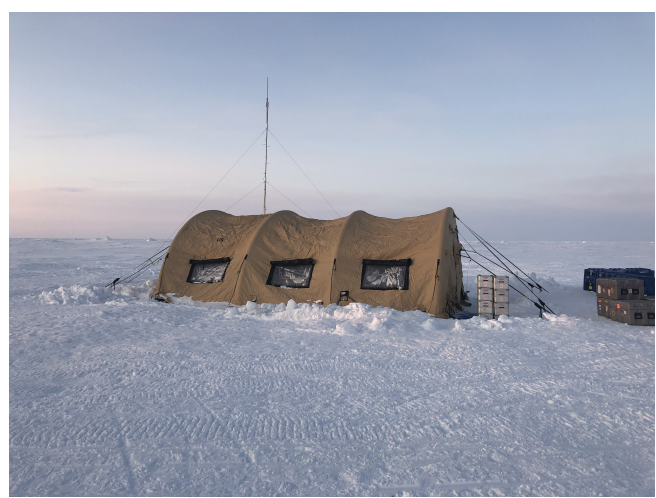

(a) Operations tent.

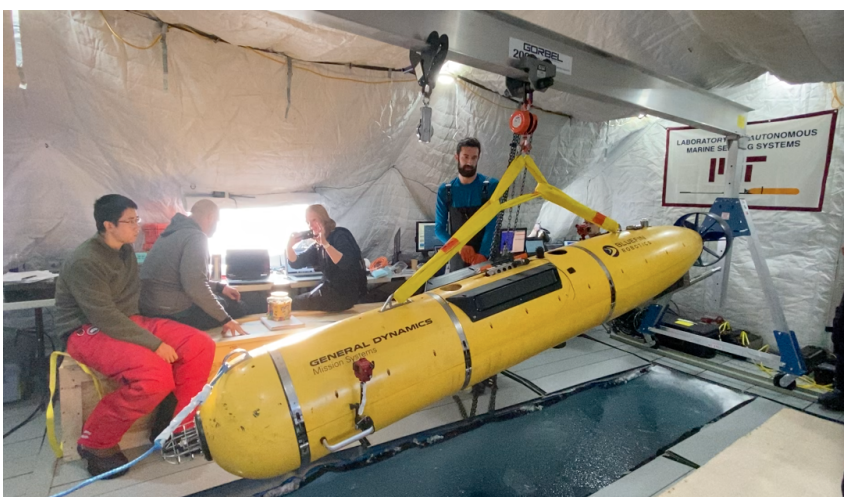

(b) Hydrohole, inside the Operations tent

Figure 3-3: Topside operations tent and the hydrohole.

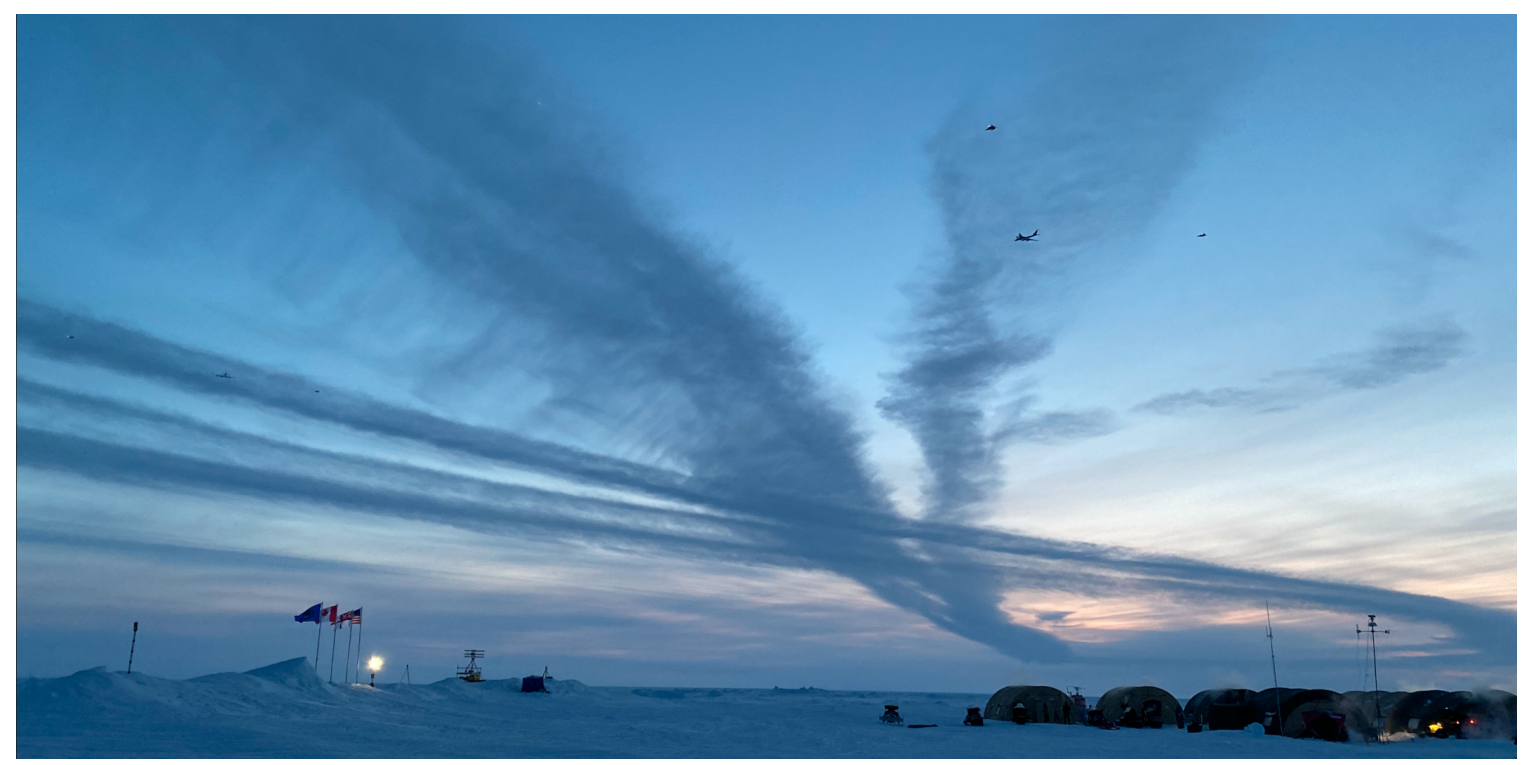

Figure 3-4: Two TU-142 Russian reconnaissance aircraft overflying Ice Camp Seadragon while flanked by F-22s and FA-18s.

\subsection{Preparations}

The preparations for ICEX comprised of multiple lines of effort, however I'll focus on the lessons learned from three areas:

1. Multiple organization coordination.

2. MIT Macrura vehicle autonomy testing at sea trials.

3. Personnel training and preparation.

The coordination efforts for the planned science operations were between MIT, WHOI, 
MIT-LL, Bluefin, ONR (Office of Naval Research), UWDC (Undersea Warfighting Development Center), COMSUBDEVRON FIVE, \& UUVRON ONE. ONR was the sponsor funding the experiments, and UWDC was responsible for setting the Navy priorities throughout the experimental time on the ice. MIT-LL, MIT, and WHOI were conducting experiments, and Bluefin is a private company that was contracted to support operations with Macrura.

\section{Front Seat - Back Seat Paradigm}

To understand Bluefin's role with Macrura, some background is beneficial. Originally when MIT-LAMSS purchased Macrura, a Bluefin 21" diameter AUV, LAMSS was responsible for the vehicle. Over multiple experiments in the early 2000's it was clear that LAMSS research objectives were stymied due to researchers spending substantial time working through vehicle hydrodynamic and stability control issues [24]. This paved the way for the shift to the frontseat-backseat paradigm [25]. The frontseat is responsible for maintaining course, speed, and depth. It is similar to what submariners often refer to as ship's control. The backseat is the place where autonomy and decision making occurs. The backseat consolidates sensor information, and the final decisions are made for the frontseat to execute in order to accomplish the mission. The backseat is what submariners think of as the Officer of the Deck or Captain.

This modular design incurs scalability risk, but within the MOOS-IvP autonomy framework, iFrontSeat is used to standardize the interface between the frontseat and backseat. This made the modular approach more efficient and scalable for unmanned undersea vehicles that support MOOS-IvP [26]. As a result, Bluefin is responsible for ensuring the frontseat is working and general maintenance of the vehicle. This freed up MIT-WHOI engineers to focus on the science and autonomy pieces.

The Main Electronics Housing (MEH) onboard Macrura is the frontseat, and the backseat is the payload which contains the autonomy software developed by LAMSS. The payload dimensions and weight are coordinated with Bluefin to ensure the hydrodynamics and buoyancy of the vehicle are correct.

\section{Lessons Learned}

- Clear expectations with defined deliverables and specific timelines are necessary to keep all parties aligned. This isn’t new, but remains important in large coordinated 
projects with multiple lines of effort.

We were at risk for missing the necessary timeline for testing Macrura in Mass-Bay prior to needing to package the vehicle for shipment to Prudhoe Bay. At the end of the day it was a contracting issue that was resolvable and should not have put the team up against the wall for testing. The final at-sea testing occurred in Mass-Bay during December 2019 where weather conditions could have easily been unacceptable for testing.

- Operational sea-trials are critically important prior to planned operations especially in unforgiving environments like the Arctic.

The sea trials paid for themselves in three key ways. First, the team found some issues in the communications between the frontseat and backseat. The vehicle was initially unresponsive to actual commands because it was bogged down in navigation communications between the frontseat and backseat. Second, many of the members on the team going to the Arctic, including the Bluefin personnel, had never operated Macrura. Sea trials provided that operating experience for all team members. Lastly, during sea trials the MEH encountered some issues requiring an internal card replacement. The entire team gained experience troubleshooting Macrura's MEH to include disassembly and reassembly which was subsequently required on the ice as well. This experience was critical to building the team's confidence and parts preparations for issues that occurred on the ice.

- Arctic safety preparations are not to be taken lightly.

In preparation for the event our team went to San Diego, and joined the professionals from ASL who conducted a substantial amount of training and preparations for the Arctic. Training ranged from the basics of logistics and what to expect while on the ice to every aspect of safety. This included symptoms of windchill to hypothermia and how to operate all the safety gear. Initially our team was a little hesitant to engage in the actual walkthroughs, but once we finally did it was beneficial. Walking out of the airport in Prudhoe Bay the temperature was around $-20^{\circ} \mathrm{F}$ with a windchill that put temperatures near $-40^{\circ} \mathrm{F}$. Watching someone build a tent or a fire is not the same as doing it for yourself, especially when the first time is at $-40^{\circ} \mathrm{F}$. Thankfully due to the expertise of ASL, those skills were never necessary on the ice, but the walkthroughs were beneficial. 


\subsection{Minor Complications}

Following the sea trials, Macrura was disassembled, shipped to Prudhoe Bay, flown to Ice Camp Seadragon and reassembled. After reassembly basic in water checks were performed to ensure the systems were functioning correctly following the transit across the country including exposure to the harsh Arctic environment. The coldest temperature the vehicle experiences in the ocean is slightly below freezing, $32^{\circ} \mathrm{F}$. When out of the water the vehicle is exposed to the Arctic environment.

The basic tests are conducted close to the hydrohole with Macrura tethered to ease recovery efforts at the completion of testing. The close runs were key to establishing real world system operating confidence prior to performing un-tethered runs. Throughout the testing phase, some minor problems occurred:

1. $25 \mathrm{kHz}$ modem physical damage

2. Main Electronics Housing card failure

3. LAMSS Hard Drive Failure

\subsection{1 $25 \mathrm{kHz}$ Modem physical damage}

There is a $25 \mathrm{kHz}$ modem that is a backup communications modem for the vehicle in the event that the $10 \mathrm{kHz}$ modem (mounted on the bottom) fails. The backup modem is critical to limit risk while operating in the Arctic. Failure of the $10 \mathrm{kHz}$ modem could result in losing the entire vehicle without backup communications. Figure 3-5a shows the position of the $25 \mathrm{kHz}$ modem on the vehicle, and Figure 3-5b shows the original mounting position for the transducer.

While recovering the vehicle during one of the tethered runs, the $25 \mathrm{kHz}$ transducer was missing. After pulling the vehicle out of the hydrohole, the transducer was no longer protruding as seen in Figure 3-5a. We assumed the transducer snapped off after coming into contact with the ice during vehicle recovery since the vehicle is positively buoyant. Luckily when we took the case off the vehicle, we noticed the transducer was still present inside the vehicle casing. Professor Henrik discussed the importance of a backup system in this setting, and after testing the transducer to ensure it still worked, we attempted to re-mount it. 


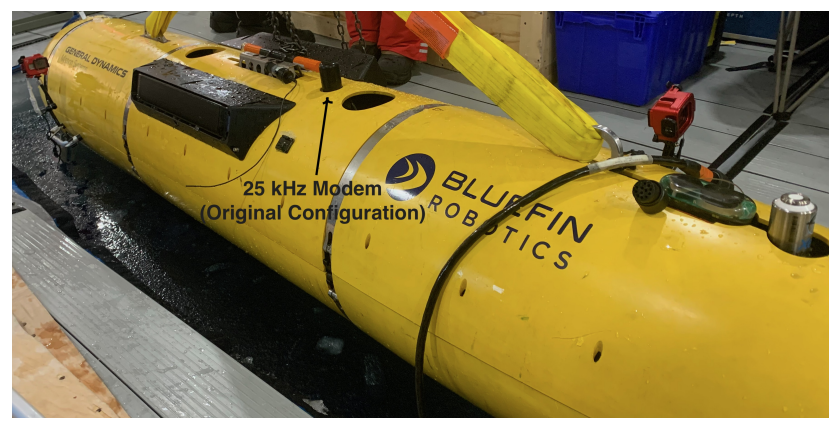

(a) $25 \mathrm{kHz}$ transducer original configuration.

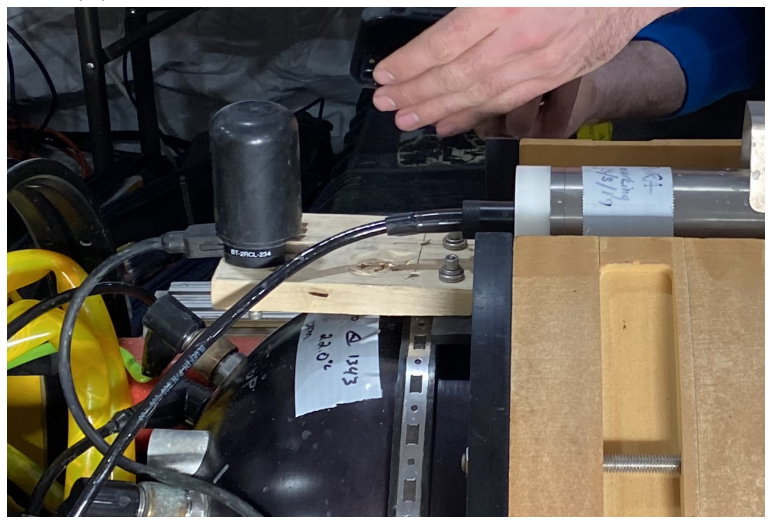

(c) Transducer mounted with wood.

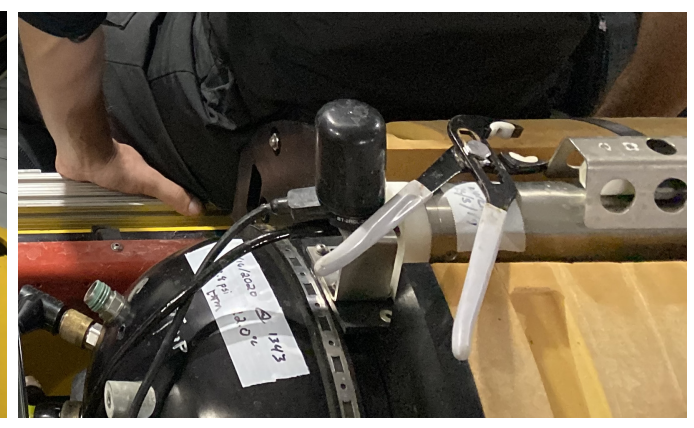

(b) $25 \mathrm{kHz}$ transducer original mount.

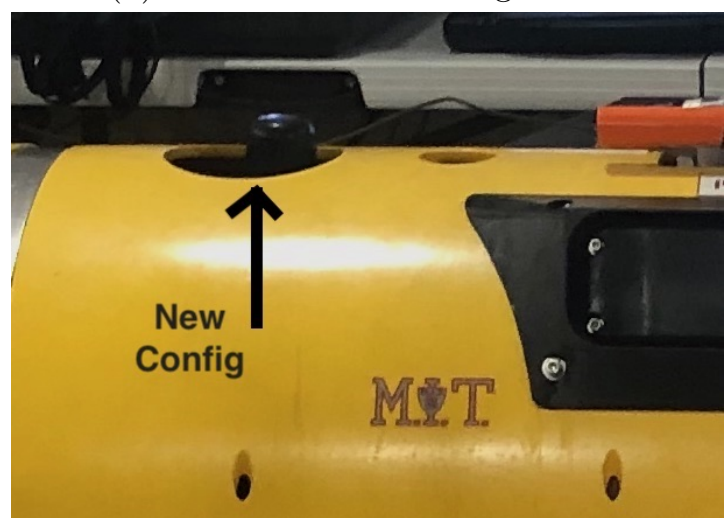

(d) $25 \mathrm{kHz}$ transducer new configuration.

Figure 3-5: $25 \mathrm{kHz}$ modem incurred damage following basic operations testing, and the mount was destroyed. The transducer was re-positioned for further operations taking advantage of wood chips that were available at the ice camp.

Although the original mounting bracket was destroyed, the bottom screw-mounts to the frame were still intact. This was also lower than the previous connection and would likely prevent future damage to the transducer during recovery when the vehicle comes into contact with the ice. The complication was the vehicle CTD connection didn't work with the 25 $\mathrm{kHz}$ modem connected directly to the vehicle frame without a mounting bracket. Figure 3-5c shows how we cut out a piece of wood to mount the transducer without interfering with the CTD.

Conveniently there was another hole in the vehicle case a few inches behind the original $25 \mathrm{kHz}$ modem hole. Originally this was for the radio frequency (RF) antenna, but that was removed for ICEX. So we employed that hole for the transducer. Moving it back a few inches, as seen in Figure 3-5c provided clearance from the CTD cable, and allowed it to go through the larger hole behind its original hole. 


\section{Lessons Learned}

- Some things you can't adequately prepare for, and the team needs a dynamic approach to problem solving while in the field. Especially in remote settings that are isolated from normal methods of communications and resources.

\subsubsection{Main Electronics Housing (MEH)}

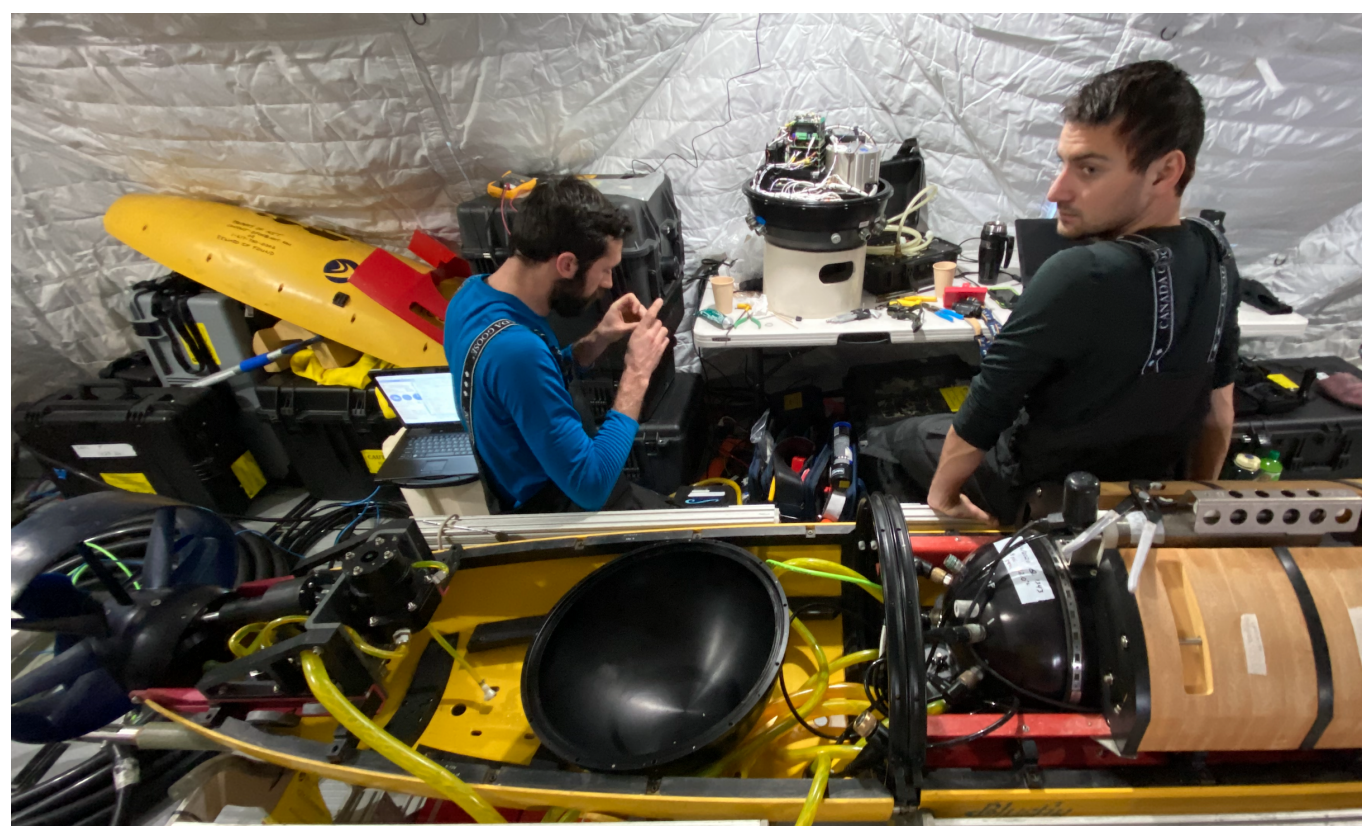

Figure 3-6: Troubleshooting and replacing a card on the MEH.

Similar to the problem encountered during sea-trials, another issue with a card on the MEH presented itself. Due to the team's experience during sea trials, it was easy to troubleshoot, isolate, and replace the faulty card. Figure 3-6 shows the MEH spherical housing open with electronics exposed inside the operations tent while troubleshooting.

\section{Lessons Learned:}

- Without the appropriate tools and parts on the ice, breaking and drawing a vacuum or card replacement on the MEH would not be possible.

- Sea trials experience was critical in building team experience and confidence troubleshooting so nobody gave up when things got challenging and ensuring the correct tools were packed for ICEX. 


\subsubsection{LAMSS Hard Drive Failure}

Following the $25 \mathrm{kHz}$ modem repairs, and $\mathrm{MEH}$ card replacement, we were excited to do a final round of testing for the day, but the payload was suddenly unresponsive. We were unable to get the LAMSS autonomy software to work. After breaking vacuum and further troubleshooting on the payload we isolated the problem to a corrupt hard drive. The LAMSS autonomy software was rebuilt on a new hard drive and the vehicle was ready for operations the next day.

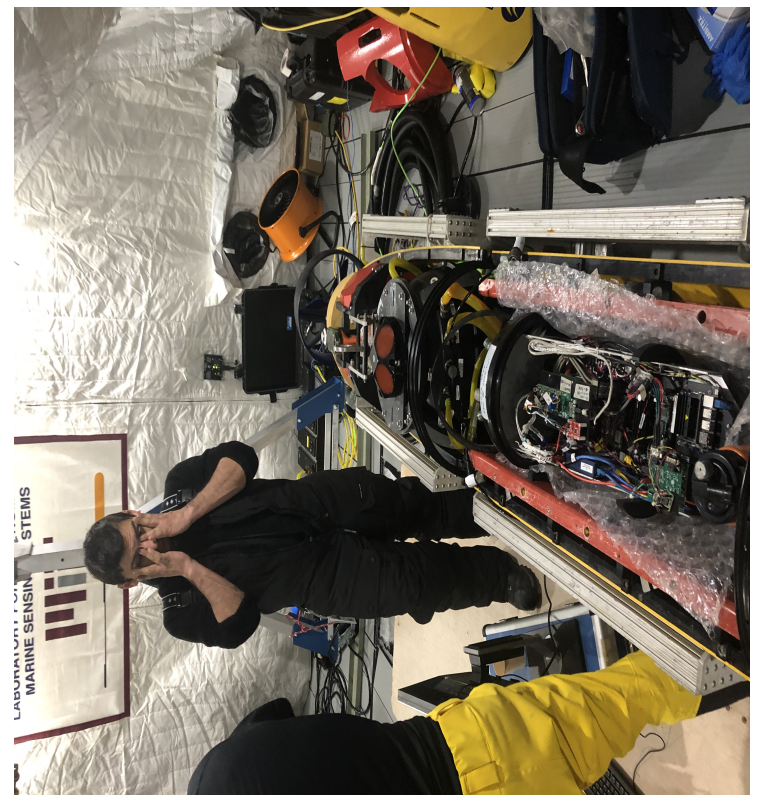

(a) Payload opened for analysis. Requires breaking vacuum to open the pressure boundary.

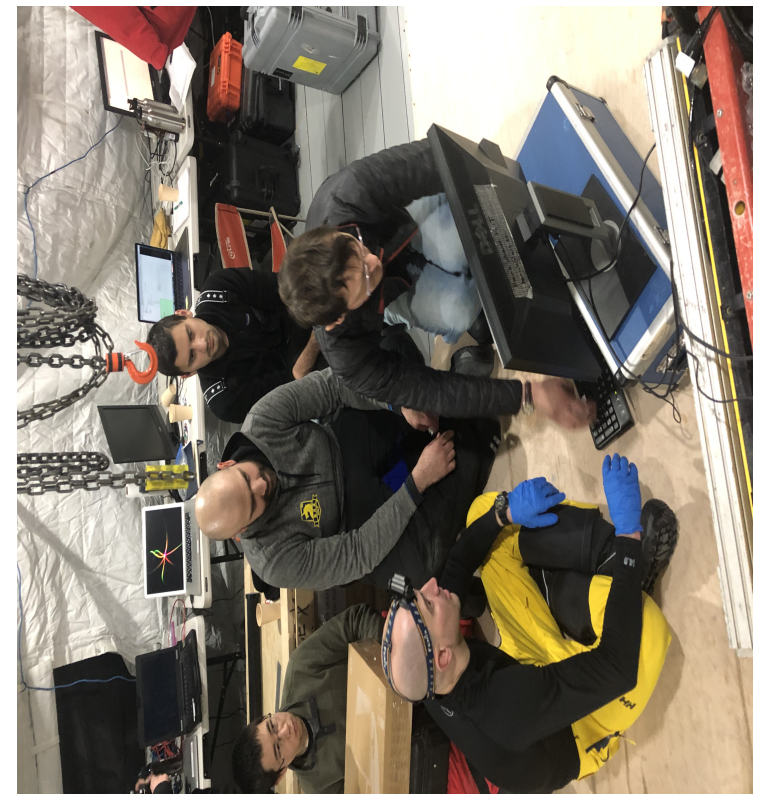

(b) Team realizes the hard drive is corrupt and needs to be replaced. Formatting new hard drive

Figure 3-7: LAMSS hard drive failure and reformatting a new disk.

When the first problem with the vehicle occurred, half the team focused on disassembly and troubleshooting, while the others came up with tests to maximize the data collection opportunities in the Arctic. During the tests, a single buoy was simulated as the vehicle and communicated with the other buoys like Macrura would. To retain four buoys as receivers, a temporary transducer was lowered into the hydrohole to act as the fourth buoy. This data set was key to validating the accuracy of our navigation approach with a direct comparison to GPS since the buoys had GPS throughout this experiment [27]. Elastic thinking enabled alternate testing while repairing the vehicle. This prevented losing valuable time in the Arctic while resources were already in place. 


\section{Lessons Learned}

- Parts and tools on the ice were invaluable to breaking, and drawing vacuum on the payload as well as an additional hard drive that was reformatted with the LAMSS autonomy software.

- When possible allocate resources to back-up plans so that when the primary objective is not executable an alternate objective can be met or accomplished.

\subsection{Major Complications}

Following the repairs, the team was prepared for the next day of operations. The first evolution was the Vertical Run as shown in Figure 1-11. This DURIP towed array data was used with this run for analysis. Next the navigation range was tested with the vehicle operating but tethered, and we practiced vehicle recovery with the ROV. Once we validated the range and successfully recovered Macrura with the ROV a few times, professor Henrik decided to go forward with the un-tethered run. This was a key decision because it was the final opportunity to show the vehicle navigation in the Arctic during real operations.

\subsubsection{Vehicle Fault}

The final run went perfectly until the the end when the vehicle was headed back toward the hydrohole. While making preparations to recover the vehicle with the ROV as we'd practiced earlier, roughly $1000 \mathrm{~m} \mathrm{NE}$ of camp, the vehicle stopped and surfaced into the bottom of the ice. From topside we were still communicating with the payload, and receiving the correct responses, but none of the commands between the backseat and frontseat appeared to be working. We weren't able to tell more about the problem, but it was quickly clear that a different recovery plan was necessary since the vehicle was not restarting.

\subsubsection{Immediate Position Mark}

While a portion of the team was developing a plan to recover the vehicle, the most important and pressing need was to immediately identify the vehicle position. This was complicated because it was nearing the end of the daylight. For personnel safety in the Arctic we don't leave the ice camp after dark because it's too dangerous. The poor visibility combined with 
the cold leads to unnecessary risk. However, it was important to mark the position of the AUV topside while in communications with the vehicle and in possession of an accurate vehicle position. It was unclear how long the vehicle battery would continue to provide power for communications, but we knew it wouldn't last until morning. Marking the ice topside was the only option since the Lat-Long position for the vehicle wouldn't necessarily be accurate in the morning due to ice motion.

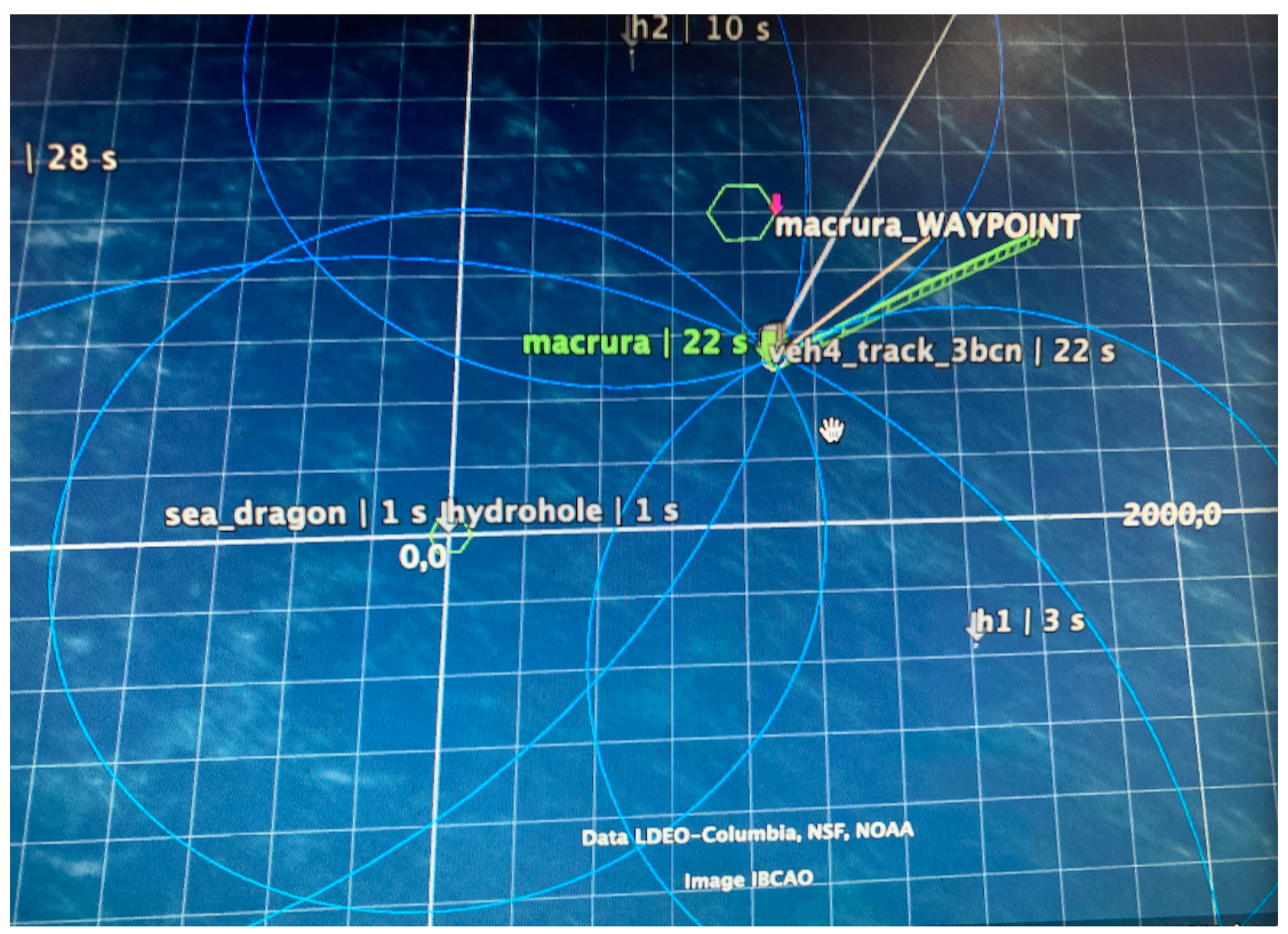

Figure 3-8: Final fix that was used to go mark the vehicle location. This was a 5-node fix since the portable hydrophone (ie. Towfish) was lowered into the hydrohole to maximize accuracy.

Consider this: If the ice were moving at 0.5 knots, and we lost comms with the vehicle 1 hour before we went out on our search party, the Lat-Long would be $0.5 \mathrm{NM}$ (>900 m) off from the actual position of the vehicle. This may not seem like much, but considering the work to search a given position on the ice doesn't entail just showing up at that location. A team must be present for safety risks, and a hole must be drilled through the ice with an auger, and a camera dropped down to look around in the water. Best case visibility is around $100 \mathrm{~m}$, and is more realistically around 30-50 m. It's clear that any error in position 
quickly becomes unmanageable. It's similar to the problems encountered in ICEX 2018 while attempting to recover the practice torpedoes.

That evening of 03-11-2020 around 1915 AKDT, as the sun was setting ( 1934 AKDT), we went out to the latest GPS position of the vehicle and marked the ice with a big "X" so we could return in the morning and begin our search for the vehicle. The vehicle battery lost power around 03-12-2020 at 0200 AKDT. The search team didn't leave to look for the vehicle until around 0900 AKDT.

\subsubsection{Vehicle Verification and Daisy Chain Recovery Method}

Over the night we developed a Daisy Chain Recovery plan. If we knew where the vehicle was, we would dig a hole approximately $300 \mathrm{~m}$ from the vehicle location in the direction of the hydrohole. The hole needed to be $\sim 14 "$ diameter for the ROV to fit. The limit of the ROV based on the electrical cabling, and spool of line was $\sim 350 \mathrm{~m}$. Once the vehicle was clipped by the ROV, we would bring them both back to the 14 " hole.

Next another 14" hole would be drilled for the ROV 300 $\mathrm{m}$ from the first hole. The ROV would then go to the first hole and clip off the vehicle again. This is where the problem arose. There were only two clips, and two lines that the ROV could use to connect to Macrura. By daisy chaining, we'd only be able to get the vehicle a total of $700 \mathrm{~m}$, and that would be with perfect conditions considering the $350 \mathrm{~m}$ constraints of the electrical cabling for the vehicle and our nylon lines. We needed to figure out a way to be able to re-use one of the clips and lines.

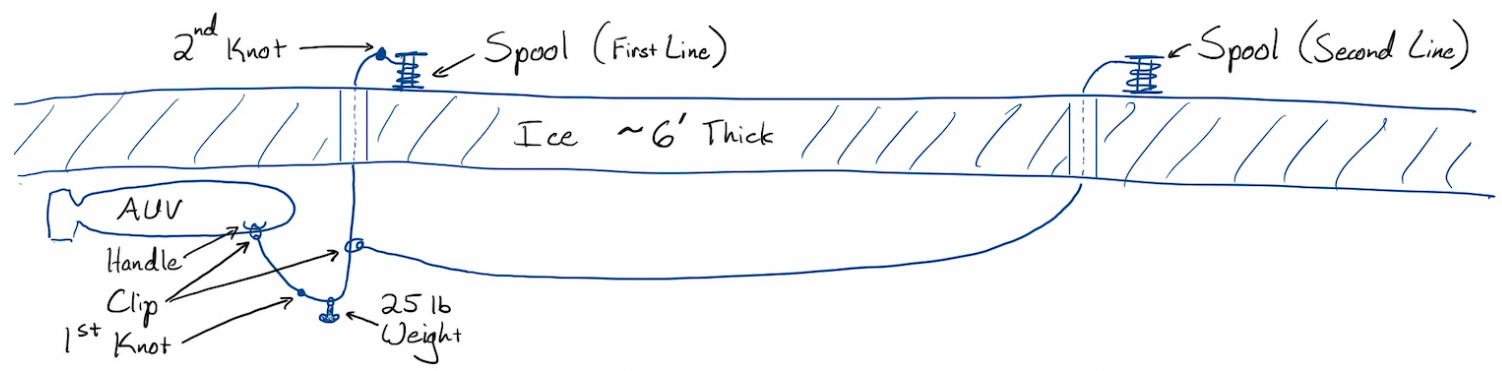

Figure 3-9: Image used to brief team on the Daisy Chain recovery plan and how the second line and clip would be re-used.

So, once the vehicle was pulled to the first $14 "$ hole, the plan was to tie a large knot in the line, then connect one of the $25 \mathrm{lbs}$ weights so the line would sink. This would create sufficient tension between the weight and the people topside for the ROV to connect the 
second clip to that line vice the vehicle handle. After the vehicle connected the clip to the line, personnel at the first hole would tie off another large knot in the line, and send the entire line into the hole. Now the people at the second hole were pulling the second line which was connected to the first line and not sliding due to the second knot tied in the line. Figure 3-9 shows a basic diagram of the setup and is how we briefed the plan. Then at the second hole they would pull the second line all the way out to re-use the second line and clip. Once we got the vehicle back to the hydrohole we could pull the 25 lbs weight out of the water since the first knot would catch the weight and pull it up. As well, the vehicle was buoyant enough that the 25 lbs weight wouldn't sink the vehicle.

\section{Victories and Complications}

The next morning we went out to the location marked the evening before to execute the plan. We used the last Lat-Long from 0200 AKDT, and relative position of roughly $045^{\circ}$ and $1000 \mathrm{~m}$ from camp to find the " $\mathrm{X}$ " from the night before. Once there, we dug a 6" hole and placed a camera under the ice to verify the vehicle was present. Incredibly, the vehicle was no more than 3' from where we'd put the "X" the night before. Our entire recovery plan hinged on actually finding the vehicle in the morning, and that first hurdle was complete.

Attempting to dig a 14" hole for the ROV to go though was not realistic. After attempting for nearly one hour, the team realized this wasn't a feasible plan. Even after the first hole was complete, getting the ROV to operate in the exposed Arctic environment wasn't executable. The ROV equipment was operating intermittently since it was no longer in the environmentally controlled operations tent. As well, the hole started to re-freeze which complicated ROV removal from the hole. We were not able to drive the ROV over to the vehicle. We returned to camp in order to build a new plan for vehicle recovery. The first problem was that there wasn't a 12" or 14" auger to drill a hole that the ROV fit through. Trying to use the 6" auger to drill multiple holes by one another was incredibly arduous, time consuming, and didn't seem executable. The second problem was operating the ROV without the environmental protections afforded by the operations tent.

Upon returning to camp, we were informed the incoming storm was getting stronger and everyone was evacuating camp with a few exceptions of key personnel. After further discussions, the command hut gave us an additional hour to attempt to secure the AUV to the ice. It was unclear when conditions would support returning to the ice for vehicle 
recovery. During that entire time, the ice and vehicle would be moving. It was necessary to tie off the vehicle topside to ensure we didn't lose it. The relative vehicle position from camp was our backup plan if were were unable to secure the vehicle and tie it off topside.

Upon returning to the vehicle location, we redug the camera hole from the morning since it had frozen. After verifying the vehicle position, we dug a second hole directly over where we thought the front of the vehicle was with the handle. In this hole we used a boat hook with a clip at the end, and incredibly on the first try connected the clip to the vehicle handle. We verified the connection to the vehicle with the camera. Next we left the spool and a 25 lbs weight on top of it topside. The hope was that at the end of the large snowstorm we would find the spool and weight.

\subsubsection{Final Recovery Planning}

Once safely in Prudhoe Bay we hunkered down for the large snowstorm. A majority of the MIT-WHOI team left that evening prior to the storm arriving in Prudhoe Bay. I remained with the team from Bluefin to ensure vehicle and data recovery. The next couple days, the town was in a severe weather condition that directed all personnel to remain indoors unless they were apart of emergency services or a two vehicle convoy.

During this time we met with members from ASL and built a plan to recover the vehicle upon returning to the ice. Each party's expertise and constraints were considered as we planned the vehicle recovery. The plan required the 36" auger to drill through the ice and recover Macrura from its resting place under the ice while exposed to the harsh Arctic environment. This meant good weather was required, and an efficiently executable plan was necessary since the team would be remote from the safety and resources at ice camp. Finally we sat down and walked through a procedure that incorporated all of the constraints to make a viable vehicle recovery operational plan.

\subsubsection{Vehicle Recovery Procedure}

On 15 March 2020, three days after departing the ice camp, we returned to Ice Camp Seadragon. The entire recovery procedure was predicated upon finding the spool left behind that was tied off to the vehicle. The spool worked perfectly, and was so obvious that a portion of the blue spool was visible from the airplane when flying into camp. Although snow piled up on one side of the spool, the other side remained visible and was a perfect 
indicator for finding the vehicle.

Consider this: Our backup plan was to use the relative position and range from camp. The location that we went to recover the vehicle was roughly bearing $090^{\circ}$ at $1100 \mathrm{~m}$ from camp. A significant difference than the original $045^{\circ}$ and $1000 \mathrm{~m}$. Again, without securing the vehicle topside prior to leaving the ice, finding the vehicle after the snowstorm was highly improbable.

Appendix A includes additional images from the vehicle recovery operation.

\section{Recovery Required Gear}

6" Auger, 36" Auger, and 18" Chainsaw

Tri-Pod, 2x Chain Falls, L-Bar \& Ice-Screw

Aqua View Camera with post for dropping through ice to view vehicle orientation.

\section{2-Hole Vehicle Recovery Procedure}

1. Locate vehicle and determine vehicle orientation

- Vehicle is tied-off to spool of blue nylon cable with 25 lbs weight on top to hold it down through the snowstorm.

- 6" auger for the camera to validate vehicle presence and orientation.

2. 58 " from the front of the vehicle use the 36 " auger

- Drill first 36" hole

- DO NOT BREAK THROUGH THE ICE YET

3. Chainsaw a "V" shape opposite the vehicle

- Put in Ice-Screw before cutting this "V" out (prevents ice from falling into the hole).

- Hole remains dry.

- This V-Cut is for the nose of the vehicle and critical to reducing the recovery angle on the vehicle.

4. 30 " from the front of the vehicle use the 36 " auger 
- Second hole is 28 " from center of first hole

- This goes all the way through the ice.

5. Complete digging out first hole.

- Water now present in hole, however only drilling through the last few inches of first hole.

6. Reposition Tri-Pod w/ CENTER at FRONT of hole (where "V" cut occurred).

7. L-Bar with AquaView:

- Use camera to watch

- L-Bar connect to either: 1. Nose top handle. OR 2. Handle on side of vehicle.

8. Once vehicle is secured through hole: Auger a 6 " hole through the blue nylon line that the vehicle is presently tied off to.

- Cuts the blue line or frees it from the ice.

- Caution not to get auger caught in the blue line. May wrap itself up and potentially damage the auger.

- Vehicle is no longer held by blue line. Must have vehicle secured topside through the recovery hole.

9. Align and pull vehicle with the nose aligned with the front of the hole.

- Disconnect nose clip with blue line (If possible)

- Connect shackle and chain fall to FWD weight bearing connection.

- NOT the handle on the nose of the vehicle. Not designed for weight bearing.

10. Lift vehicle out of water from FWD lift point to gain access to the Aft Lift Point.

11. Connect clip with shackle to the Aft chain fall.

- Use boat hook to connect when the aft lift point is visible.

- Transfer load from fwd to aft chain fall to balance loading and continue to lift vehicle out of the water. 
12. With vehicle entirely out of water $\approx 2$ " or more:

(a) Place plywood over hole $\rightarrow$ Remove DURIP towed array.

(b) Place sled beneath vehicle.

(c) Carts are on sled. ** Worst case plan for no vehicle carts.

(d) Lower vehicle onto carts.

(e) Return to ice camp and remove payload for flying back to Prudhoe Bay.

\section{Lessons Learned}

- Precise navigational accuracy was critical to vehicle recovery, and the navigational framework developed for ICEX provided that fidelity.

- Without the Arctic navigation accuracy, vehicle recovery was highly improbable.

- Navigational accuracy enabled marking an "X" in the ice at the precise vehicle location making later recovery feasible.

- Elastic thinking and problem solving was necessary to plan vehicle recovery operations, especially considering the constraints of the equipment, personnel, and environment.

- Developed and executed a procedure to recover Macrura without divers, an ROV, or a tent to conduct the operations from.

- Macrura was recovered with a roughly $53^{\circ}$ up angle without any damage to the vehicle.

- Collaboration between ASL, Bluefin and MIT enabled effective planning and execution without needing to come up with solutions in the field. Operational planning for the recovery was successful due to the team effort and unified vision in safely recovering the vehicle. 


\section{Chapter 4}

\section{ICEX DURIP Array Analysis}

\subsection{DURIP Towed Array Beamforming Specifics}

The DURIP towed array element layout is unique as seen in Figure 1-8, and described as a nested array. The larger aperture array elements are spaced at $1.5 \mathrm{~m}$ which is cut for a wavelength of $d \leq \frac{\lambda}{2} \Rightarrow \lambda \geq 2 * 1.5=3 \mathrm{~m}$. Approximating the speed of sound in water, $c=1500 \mathrm{~m} / \mathrm{s}$, the maximum frequency is roughly $f_{c u t}=\frac{c}{\lambda}=\frac{1500}{3}=500 \mathrm{~Hz}$ without resulting in grating lobes. For the smaller, nested array, the spacing is $0.75 \mathrm{~m}$. This is cut for a wavelength of $\lambda \geq 2 * 0.75=1.5 \mathrm{~m}$. Therefore, the $\max$ frequency is roughly $f_{\text {cut }}=\frac{1500}{1.5}=1000 \mathrm{~Hz}$. The particular frequency of interest is $5 \mathrm{kHz}$, the subharmonic of the $10 \mathrm{kHz}$ carrier frequency from the WHOI micro-modem buoy transmissions and the additional source energy between $5-6 \mathrm{kHz}$. In this particular case with $f=5000 \mathrm{~Hz}$ as the target frequency for beamforming:

$$
\lambda \approx \frac{1500 \mathrm{~m} / \mathrm{s}}{5000 \mathrm{~Hz}}=0.3 \mathrm{~m} \text { In this case, } \quad d>>\frac{\lambda}{2} \Rightarrow 0.75 \mathrm{~m}>>\frac{0.3}{2}=0.15 \mathrm{~m}
$$

\subsubsection{DURIP Towed Array Spatial Aliasing or Grating}

Since $d>>\frac{\lambda}{2}$ in the case of $5 \mathrm{kHz}$, the result is grating lobes. Figure $4-1$ shows what the beam pattern for the DURIP nested array looks like for a $5 \mathrm{kHz}$ signal. This is equivalent to $d=\frac{5 \lambda}{2}$. Figure $4-1 \mathrm{a}$, shows the the beams steered to $90^{\circ}$ relative, and Figure $4-1 \mathrm{~b}$ shows the beams steered to $78.5^{\circ}$ relative. From these beam patterns, it's clear that anything outside of $\pm 11.5^{\circ}$ from the beam of the array is going to be spatially aliased at $5 \mathrm{kHz}$.

Figure 4-2 shows a transmission from the WHOI micro-modem that is easily distin- 


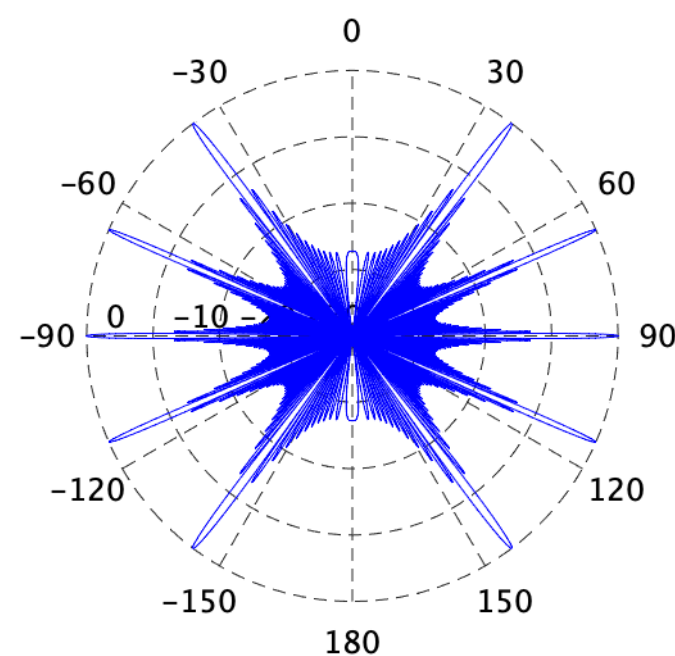

(a) Polar plot with $d=\frac{5 \lambda}{2}$. Equivalent to DURIP nested array beamformed at $5 \mathrm{kHz}$.

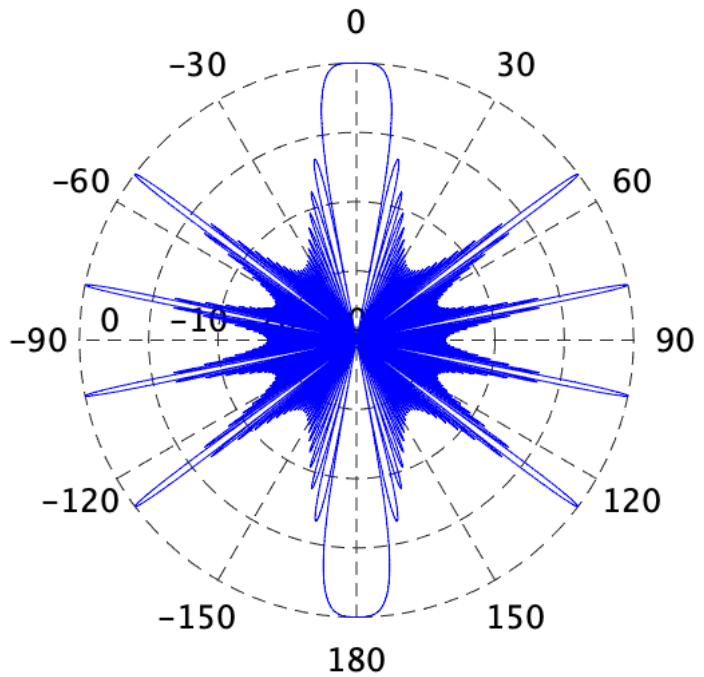

(b) Polar plot with $d=\frac{5 \lambda}{2}$ steered to $78.5^{\circ}$. Equivalent to steering to $0^{\circ}$ (endfire).

Figure 4-1: Polar plot showing grating lobes of DURIP nested array (23-Elements) beamformed at $5 \mathrm{kHz}$.

guishable. The figure labels some portions so it's easier to discern transmissions later when looking at beamformed data. There are clearly two separate arrival paths present. One arrival occurs at $\sim+4^{\circ}$ and one at $\sim+9.3^{\circ}$. The arrival at $+4^{\circ}$ is stronger than and it arrives just slightly before the arrival at $+9.3^{\circ}$. This is because the $+4^{\circ}$ arrival is direct path from the WHOI micro-modem without any other interactions before it arrives at the array. The $+9.3^{\circ}$ arrival was a reflection from off the ice.

Later in the chapter looking at the BELLHOP analysis Figure 4-30, shows these two arrival paths. The minor time delay is due to the longer propagation path for the surface reflected sound wave to travel prior to arriving at the DURIP towed array. All of the additional transmissions that were captured and evaluated look similar to this example, but not necessarily as crystal clear. The buoy transmissions are visible near the $0^{\circ}-10^{\circ} \mathrm{D} / \mathrm{E}$ in the last 2.5 to 4 seconds of each window. This true for the transmissions in this chapter and the additional transmissions in Appendix B.

Figure 4-3 shows data from ICEX. Figure 4-3a shows the DURIP towed array data processed between 500 and $1000 \mathrm{~Hz}$. This is inside the design spectrum for the array, so no spatial aliasing occurs. Although, at this frequency spectrum, the transmission from the WHOI micro-modem buoys isn't captured. Consider the PSD from Chapter 1, Figure 1-13. It clearly shows that data between $5-6 \mathrm{kHz}$ is needed to see the buoy transmissions. 


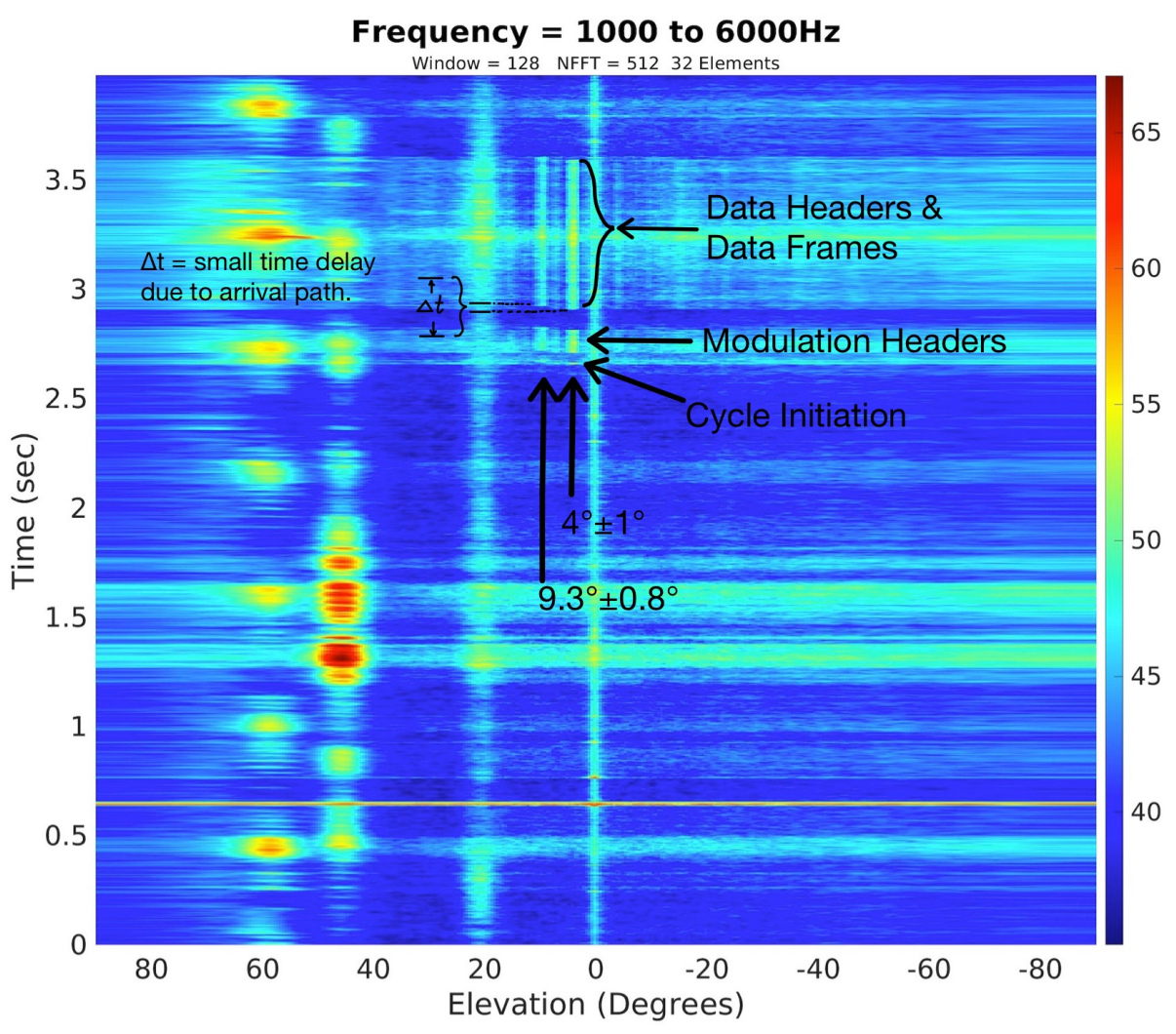

Figure 4-2: WHOI micro-modem transmission \#1893 from H1 $\left(z_{s}=90 \mathrm{~m}\right)$ captured on the DURIP towed array at a depth of roughly $159 \mathrm{~m}$. Here the direct path arrival, $+4^{\circ}$, is stronger and arrives slightly earlier compared to the surface reflected arrival which comes in at a steeper $+9.3^{\circ}$. The small time delay is due to the slightly longer path for the wave to travel upward into the ice, reflect, and arrive at the array vs. the direct path transmission.

Figure 4-3b is processed between $500-6000 \mathrm{~Hz}$. This includes the frequency content from the WHOI micro-modem transmission. Suddenly between 2.8 to 3.6 seconds, at $+9^{\circ}$ and $+4^{\circ}$ the transmission is clearly present. There is some slight aliasing visible, but since the entire spectrum from 500 to $6000 \mathrm{~Hz}$ is being evaluated, the aliasing isn't overwhelming. The strength of the buoy transmission does start to wash out some of the other beams during the transmission, which occurs anytime the array is over driven by a loud source. This is the horizontal lighter green color that happens around loud sources. It's apparent that the signal at $+9^{\circ}$ and $+4^{\circ}$ is the actual acoustic data from the experiment. See Appendix B for a further examples and discussion of spatial grating on the array.

It's also important to keep in mind that as seen in Figure 2-12, and further in Appendix $\mathrm{B}$, the beam width narrows as we increase the frequency above the cutoff frequency for the array. If we calculate the HPBW for the DURIP nested array at a frequency of $5 \mathrm{kHz}$ we 


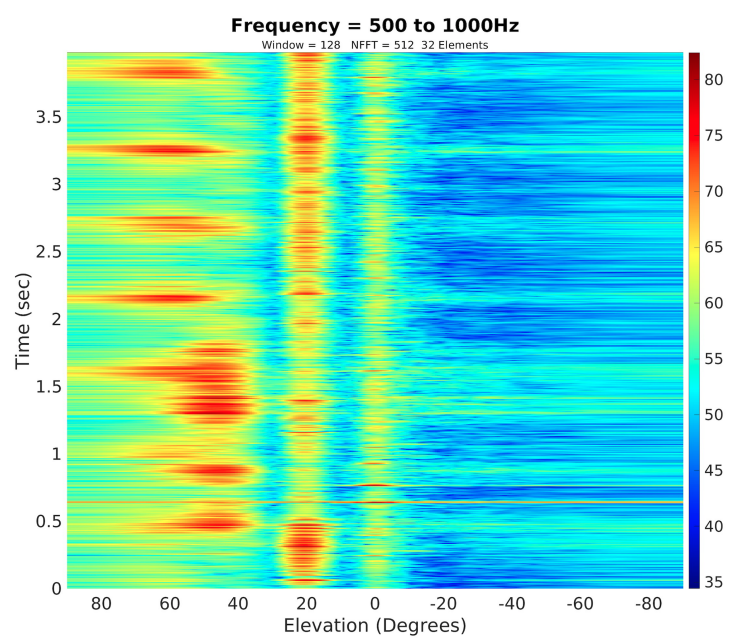

(a) DURIP Towed Array beamforming $f=$ $500-1000 H z$. Transmission \#1893.

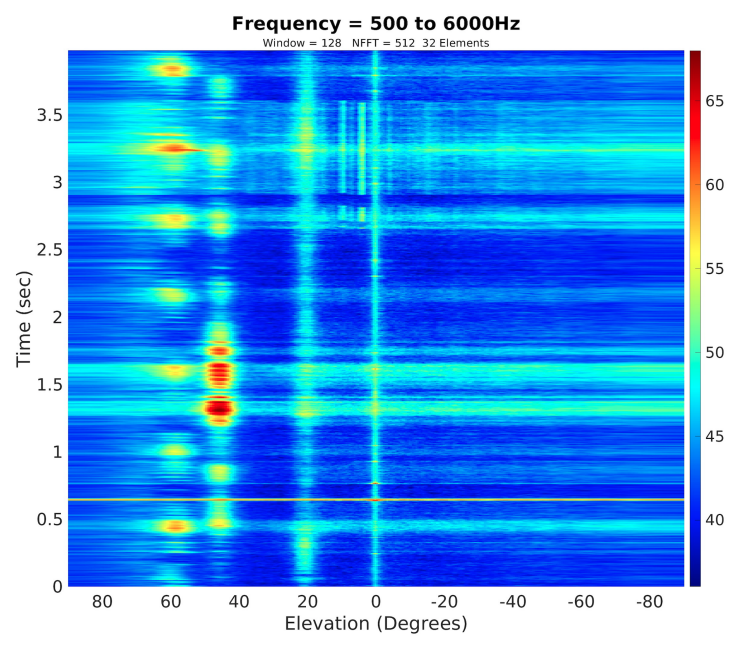

(b) DURIP Towed Array beamforming $f=$ $500-6000 \mathrm{~Hz}$. Transmission \#1893.

Figure 4-3: DURIP towed array beamforming comparison. The WHOI micro-modem transmission is not visible in $4-3 \mathrm{a}$. In $4-3 \mathrm{~b}$ the transmission is visible since this frequency spectrum includes the portion that captures the WHOI micro-modem subharmonic at $5 \mathrm{kHz}$ and additional energy between $5-6 \mathrm{kHz}$.

get the following:

$$
\cos ^{-1}\left(1-\frac{.45 * 2}{5 * 23}\right)+\cos ^{-1}\left(1+\frac{.45 * 2}{5 * 23}\right)=2 \cos ^{-1}(0.992)=14.3^{\circ}
$$

Compared to the HPBW at the beam, $\theta_{T}=90$ :

$$
\cos ^{-1}\left(0-\frac{.45 * 2}{5 * 23}\right)+\cos ^{-1}\left(0+\frac{0.45 * 2}{5 * 23}\right)=\cos ^{-1}(-.0078)-\cos ^{-1}(.0078)=0.897^{\circ}
$$

This is significantly smaller than the originally calculated values of $32^{\circ}$ and $4.5^{\circ}$ respectively. This benefit of smaller beam widths comes at the cost of spatial aliasing.

\subsubsection{DURIP Towed Array Full Beamforming with all Elements}

Another factor, based on the physical constraints of the DURIP towed array, is that if all of the elements are used, the beam pattern is not actually the same as the beam patterns developed in Chapter 2 and Appendix B. The beam patterns in Chapter 2, Figure 2-10, are based on the nested array with 23 elements cut for $1000 \mathrm{~Hz}$. Although the beam pattern is not as clean when all 32 elements are used together. Since the DURIP towed array was employed in a complex, real-world environment, the additional elements were valuable. 
Consider the BELLHOP ray traces in Figures 4-15a, 4-15b, 4-16a, and 4-16b. As the DURIP towed array moves through these dynamic environments, it's beneficial for each element to be employed in order to build the best beamformed data. In other words, it's not a perfectly homogeneous environment where the wave reaches all elements of the array equally. As a result, the additional elements are beneficial when beamforming.

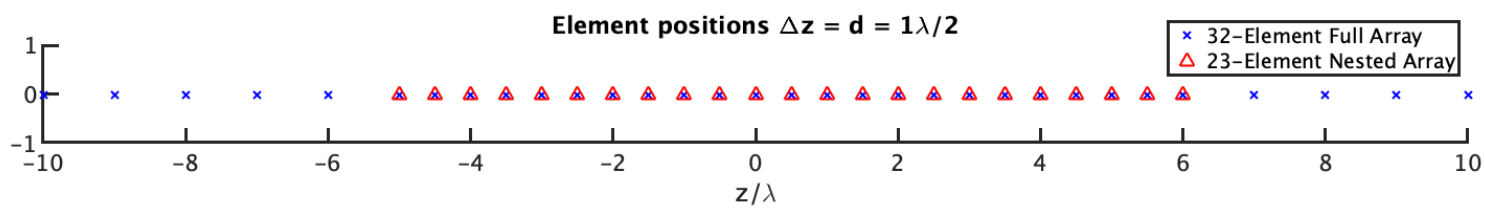

Figure 4-4: Layout of DURIP towed array highlighting the 23 element nested array in the center with red triangles.

Figure 4-4 highlights the physical elements and layout for the full 32 element array, and the 23 element nested array shown as red triangles. This unique array design layout results in a different beam-pattern than a uniformly spaced array. Figure 4-5 shows the difference in the beam pattern. There are four key differences with the 32 element array:

1. The main lobe is narrower.

2. The side lobes nearest the main lobe are suppressed, amplifying the main lobe even more.

3. The side lobes near the back lobe are much larger.

4. The back lobe is much stronger, however it is still in excess of a $10 \mathrm{~dB}$ drop.

Figure 4-6 highlights the differences between the full DURIP towed array and the nested array in u-space. It's important to look at Figure 4-6b because it helps visualize the beam pattern compression that occurs into the visible window as $f \uparrow>f_{\text {cut }}$ or when $d>\frac{\lambda}{2}$. Since the beamforming is intended to capture frequency content between $5-6 \mathrm{kHz}$, anticipating what sort of pattern to expect is crtical. Lastly, this larger perspective shows one last critical piece of information:

- The grating lobes occur at the same point with the full 32 element DURIP array as with the 23 element nested array. This is largely due to the smallest element spacing, $\Delta z$, of both the full array and nested array is the same at $\Delta z=0.75 \mathrm{~m}$. 
23 vs 32 Elements Uniform Weight with Steering Angle = 135; d = 1 $\lambda / 2$

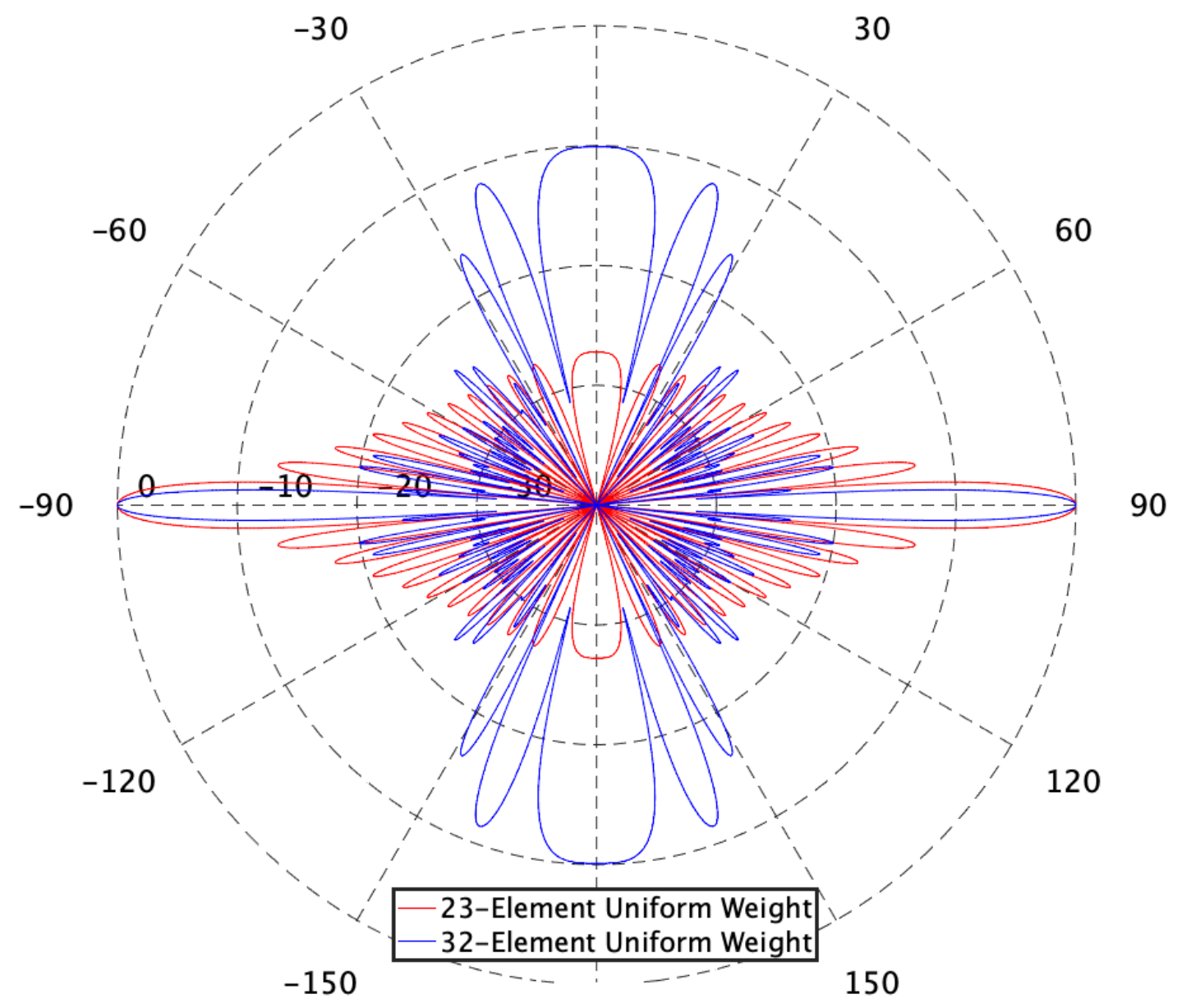

Figure 4-5: Uniform 23 element nested array vs. Full 32 element array beam pattern.

\subsubsection{Hanning Window Weighting}

Instead of a uniform weight distribution, which is akin to performing a rectangular window convolution in Equation 2.14. The complex weight vector, $\mathbf{w}^{H}$, defined in Equation 2.19 is generating a square wave with a uniform weighting. Instead a different weight vector is used. The Hanning window, named after Julius von Hann, was used which includes the added benefit of reduced discrete Fourier transform leakage [28]. The basis of the Hanning window for weighting is:

$$
w_{n}(n)=C * \cos ^{2}\left(\frac{\pi z}{N d}\right) \quad \text { where } \quad C=\text { normalization constant }
$$




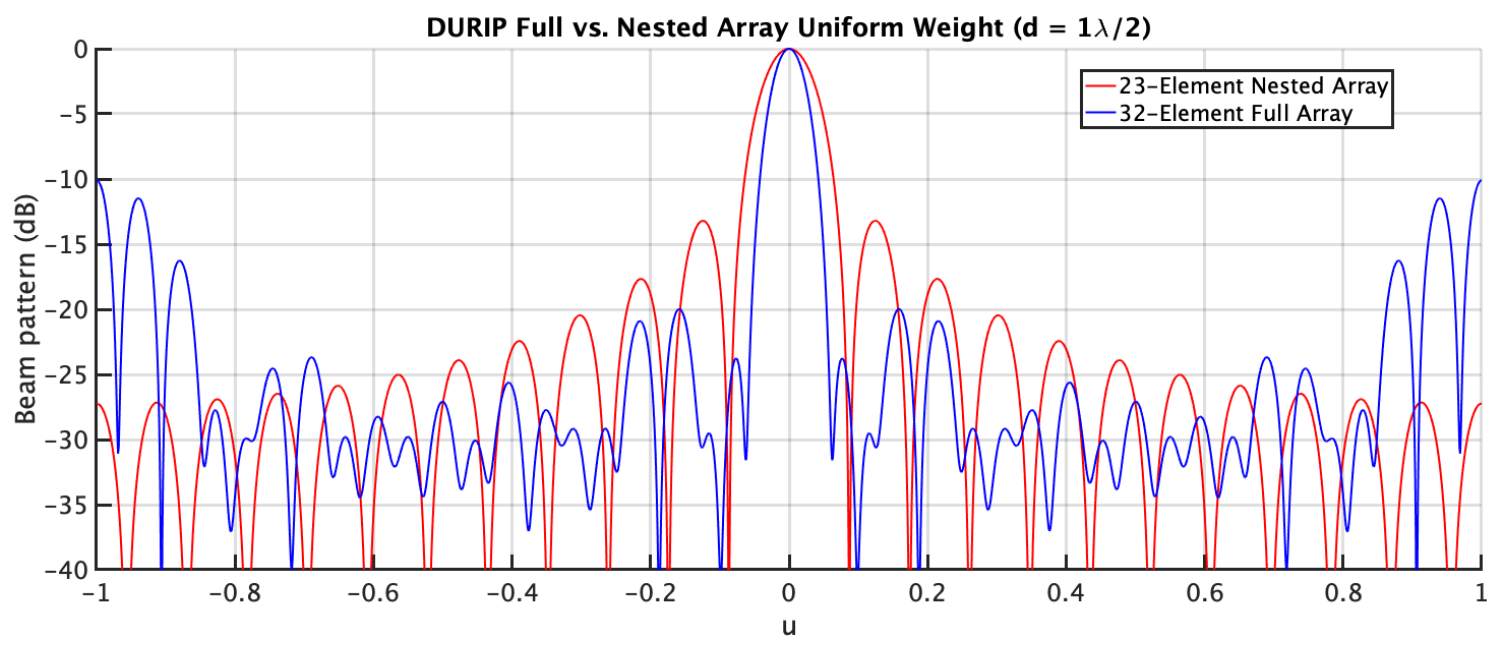

(a) u-space for the 23 element nested array vs Full 32 element array.

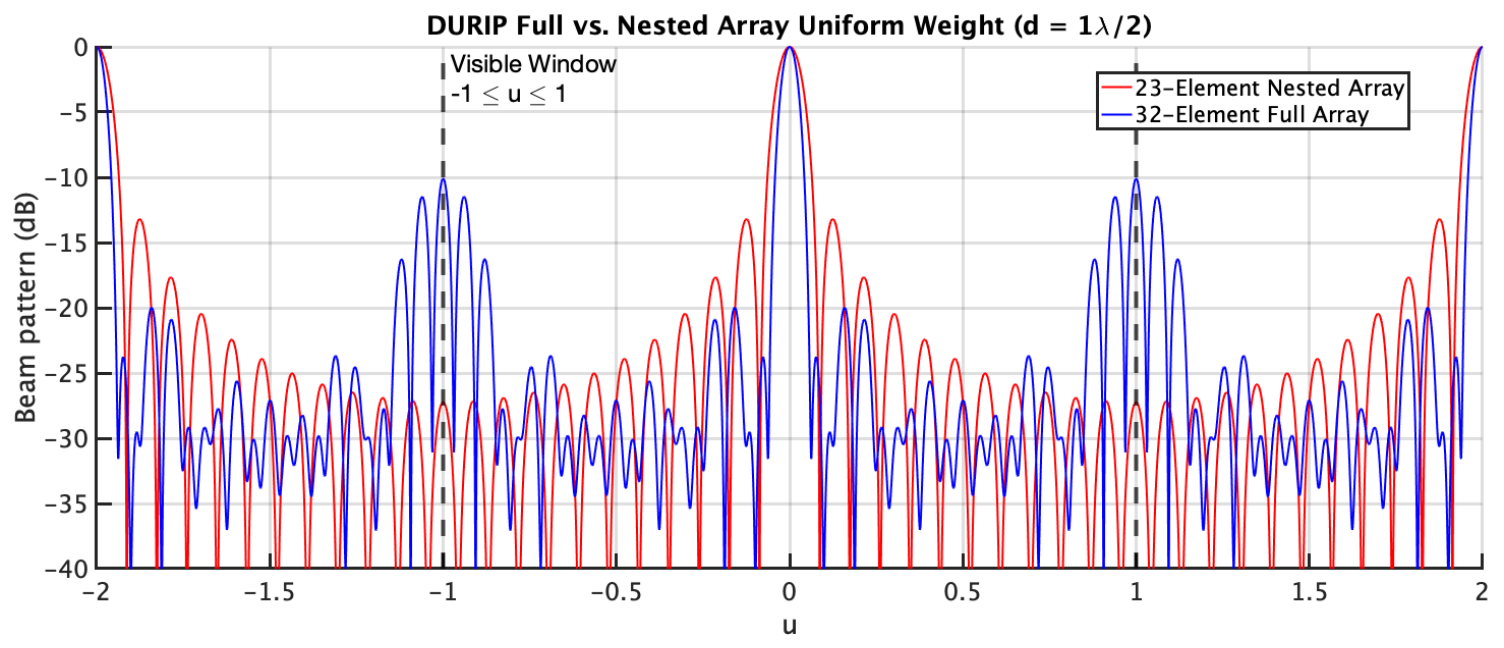

(b) u-space for visualizing u-space compression above the array $f_{\text {cut }}$.

Figure 4-6: Nested array (23 elements) vs Full DURIP array (32 elements) beam patterns with uniform weights. 


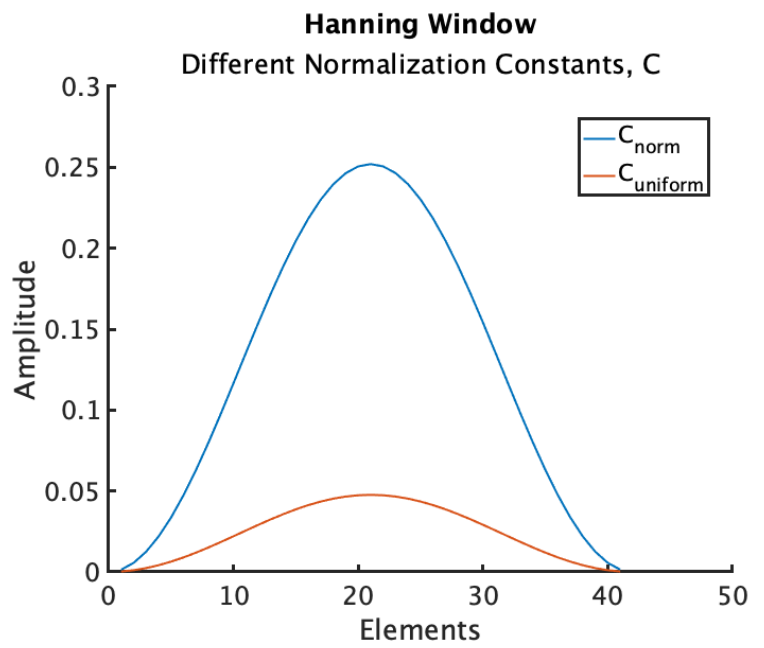

(a) Hanning window used as the array weighting (b) function.

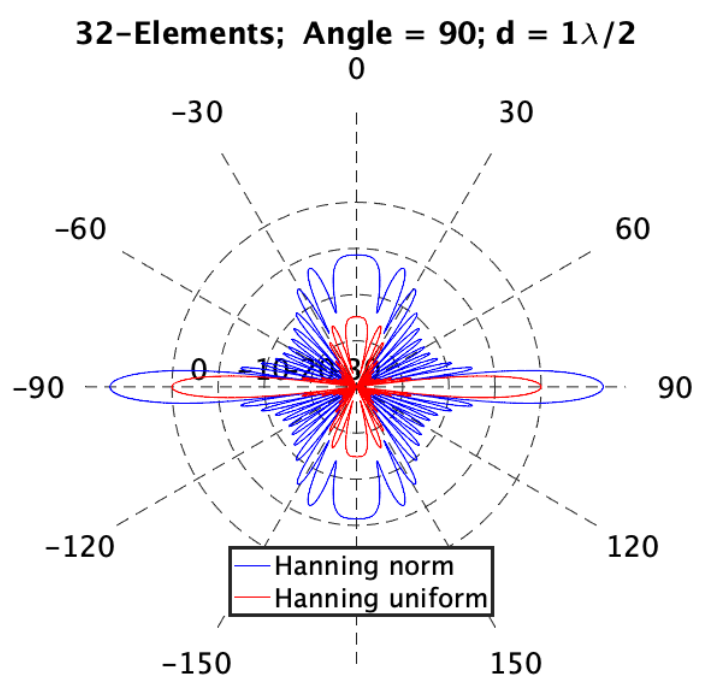

(b) Beam pattern comparison based on the normalization constant.

Figure 4-7: Plot of different normalization constants, and their impact on the beam pattern.

This analysis was done with the normalization constant, C, two different ways. Typically in digital signal processing or statistics, the preference is that the sum of the convolution window $=1$, area under the curve, to maintain continuity and prevent adding energy [29]. However, in acoustic signal processing sometimes the norm of the vector may be used as the normalization constant. Recall the norm $=\left\|w_{n}\right\|=\sqrt{\sum_{n}\left|w_{n}\right|^{2}}$. This is the difference between suppressing the background noise (area under the curve $=1$ ), and amplifying the signal through the noise $($ norm $=1)$. Both are effectively the same as seen in Figure 4-7

$$
C_{n o r m}=\frac{1}{n o r m(w)}=\frac{1}{\sqrt{\sum_{n}\left|w_{n}\right|^{2}}} \quad \text { or } \quad C_{\text {uniform }}=\frac{1}{\sum_{n} w_{n}}
$$

NOTE: If we were attempting to measure a Sound Pressure Level (SPL) at the receiver to compare to the SPL of the source and compare that to the transmission loss, we would need to ensure that we were calculating the value using the $C_{\text {uniform }}$. This is because using $C_{n o r m}$ is technically amplifying the $\mathrm{dB}$ of the received signal. Since we're just doing a comparison relative to other sound present in the environment, there is no difference other than the change in the $\mathrm{dB}$ scale.

Figure 4-7 shows that although the peaks of the Hanning weights are different (Figure 4-7a), and the resulting $\mathrm{dB}$ comparison in the beam pattern is different (Figure 4-7), there is only this relative difference. Effectively the shape of the window and resulting relative 

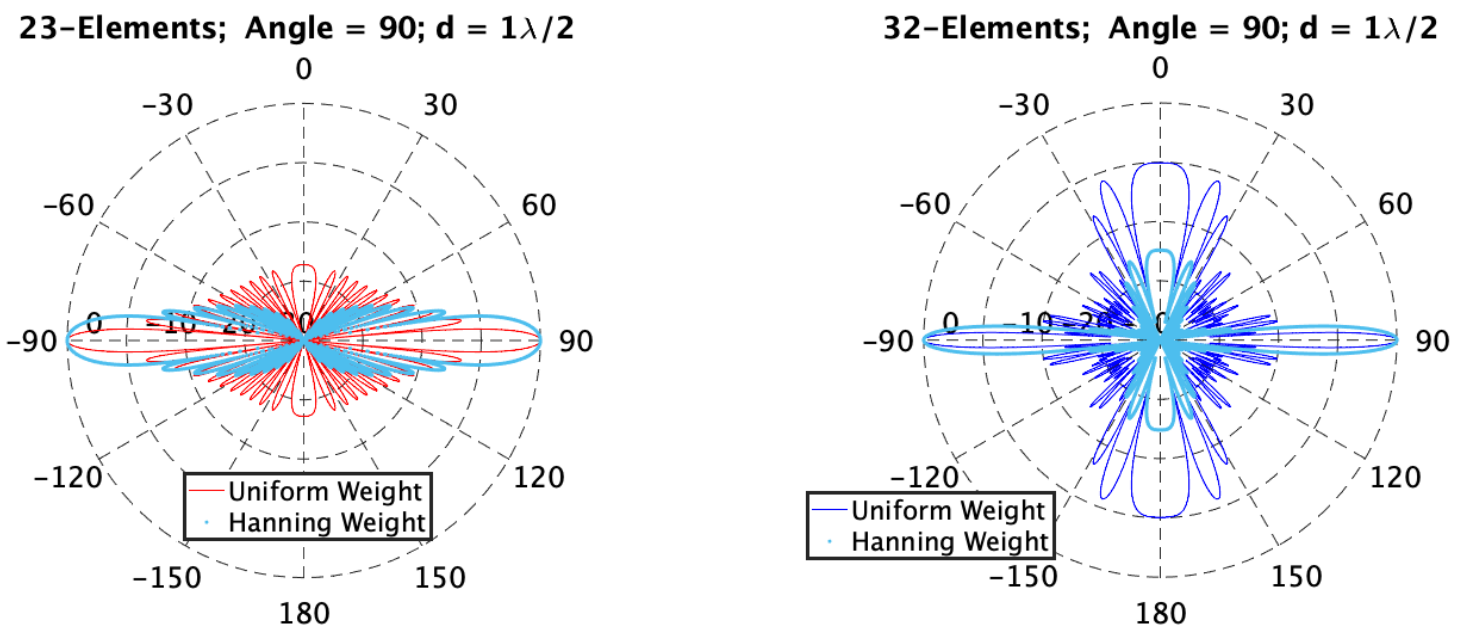

Figure 4-8: Left polar plot shows how Hanning weights affect the beam pattern of the 23 element nested array. The right plot shows how the Hanning weights affect the beam pattern of the full 32 element DURIP array.

weights are identical. Comparing the beamformed ICEX data using both normalization constants resulted in no difference with the exception of the $\mathrm{dB}$ scale. Otherwise the plots were identical and nothing could be gleaned from the difference as expected.

However, it's important to do a comparison of a Hanning window for weights to a rectangular window or uniformly weighted array. This comparison is similar to the comparison in Figure 4-5. Instead it's important to understand first, how the Hanning weighting changes the beam pattern of a 23 element uniform linear array, and the full 32 element DURIP array pattern.

It's clear from Figure 4-8 that the Hanning weights dramatically reduce the side lobes, and the back lobe is nearly non-existent. This comes at the cost of having a slightly wider beam width (eg. for the nested array, the beam width of the main lobe with Hanning weights captures the first side lobe for the uniform weights. The real advantage comes when we apply the Hanning weights to the full DURIP array. Figure 4-9 shows the comparison between beam patters of the 23 element array with uniform weights compared to the full 32 element array with Hanning weights. Using the Hanning weights allows us to take advantage of the additional array elements while maintaining the beam width of the main lobe, and significantly suppressing the side lobes of the nested array. The only cost is that the back lobe is a little stronger than the nested array

Looking at the comparison between the 23 element nested array with uniform weights 


\section{3 vs 32 Elements Different Weights; Angle $=90 ; d=1 \lambda / 2$ 0}

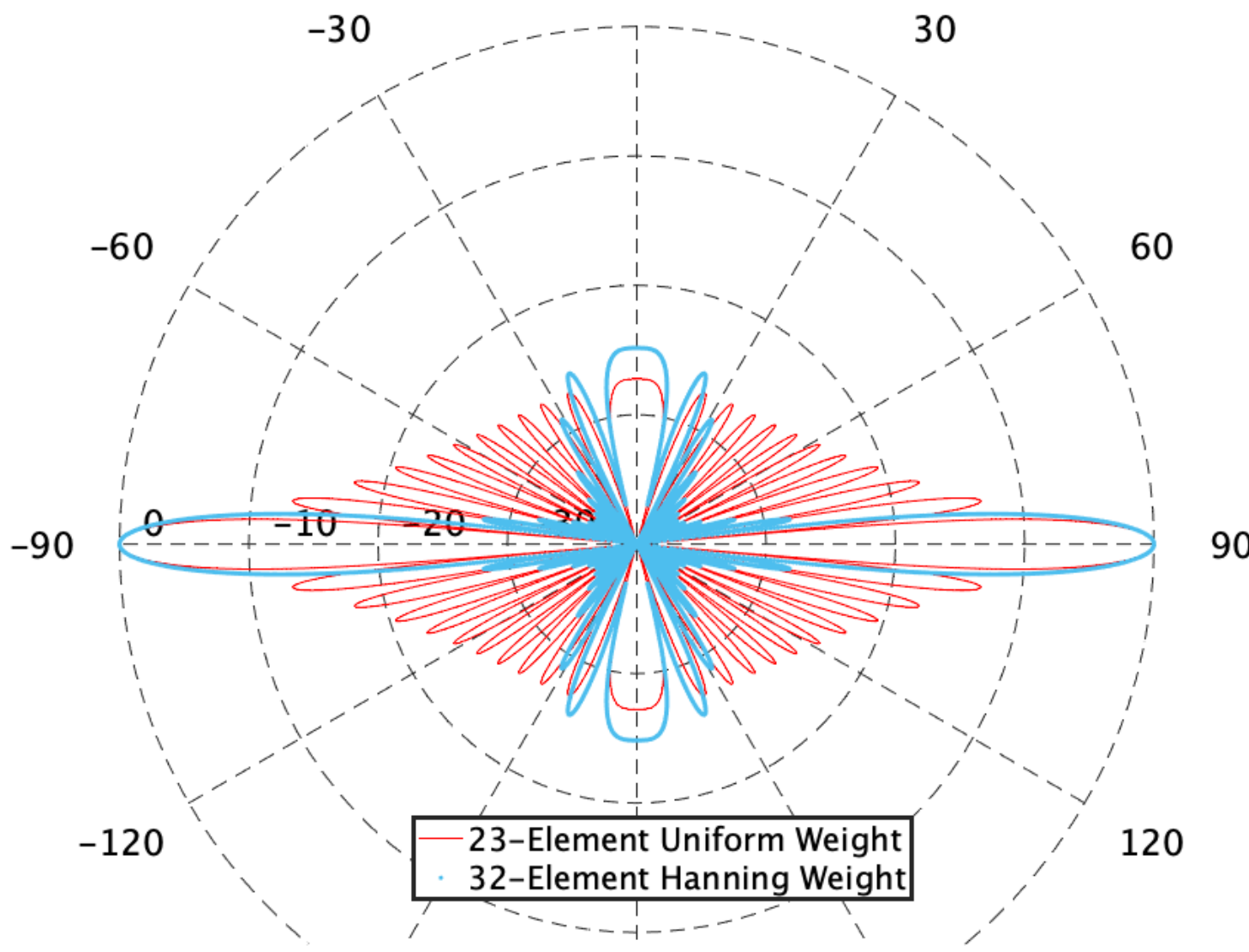

Figure 4-9: Polar plot of 23 element nested array with uniform weights compared to the full 32 element DURIP array with Hanning weights.

compared to the full 32 element DURIP array in u-space is easier to see the substantial side lobe reduction resulting from the Hanning weights, see Figure 4-10. As well it's clear that the back lobe is just barely more than the 23 element uniformly weighted array. In our real world scenario it is beneficial to have the additional elements due to the complexity of the environment.

The final decision is in comparison of the full DURIP array using uniform vs Hanning weights. The side lobe and back lobe reduction are worth the cost of a slightly wider main lobe. Figure 4-11 shows the u-space comparison.

Looking at the ICEX data for the final comparison we can make some nice comparisons. Figure 4-12 shows the sharper D/E from the narrower main lobe with the uniform weights. However the uniform weights also result in some over driving the array and there are some 


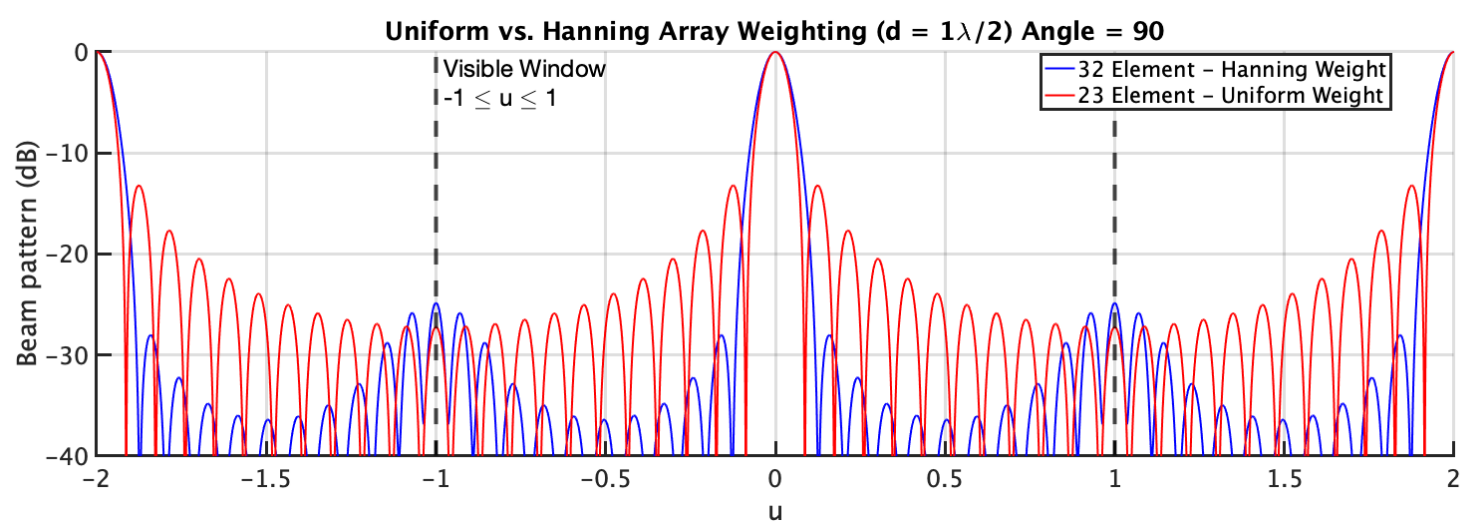

Figure 4-10: u-space comparison of 23 element nested array with uniform weights and the full 32 element DURIP array with Hanning weights.

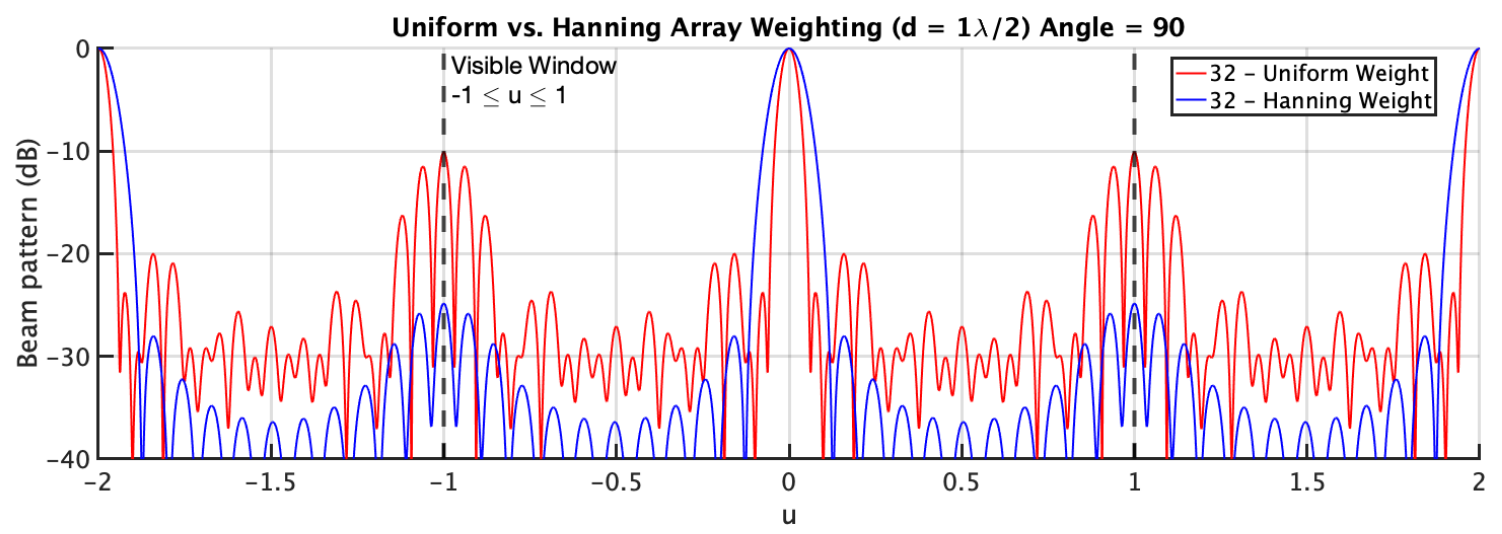

Figure 4-11: u-space comparison of full DURIP array with uniform weights vs Hanning weights. See right polar plot Figure 4-8

horizontal lines across all D/E's at a few points as a result. This is due to the much higher side lobes and back lobe for the uniform weights.

Figure 4-13 shows a different element of using the uniform weights for the full array. In this case the added benefit of a narrower main lobe is lost due to t he stronger side and back lobes which don't sufficiently suppress the background noise and subsequently the micro-modem transmission is missed.

The minor improvement in $\mathrm{D} / \mathrm{E}$ resolution due to the narrow main lobe is not worth the added cost of stronger side and back lobes. Although Figure 4-12 shows how nice the narrower main lobe is when conditions are quiet, most of the time during the experiment was fairly noisy. There was a substantial amount of background noise coming from the camp generators, helicopter operations, and snowmobiles, that the environment was rarely as quiet as during Figure 4-12. Instead, Figure 4-13 is more indicative of the level of background 


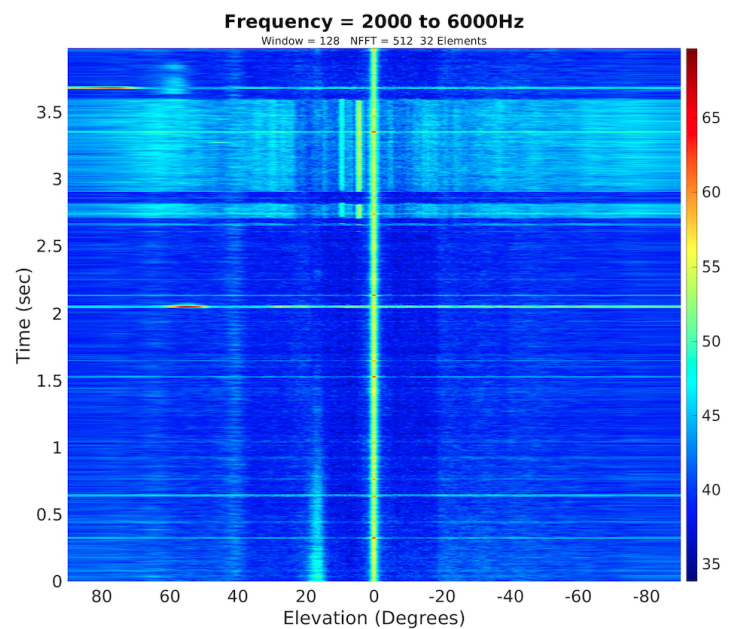

(a) Hanning weights with full DURIP array. The wider main lobe is apparent in the array noise at $0 \mathrm{D} / \mathrm{E}$ and the buoy transmission between 2.8 to 3.6 seconds.

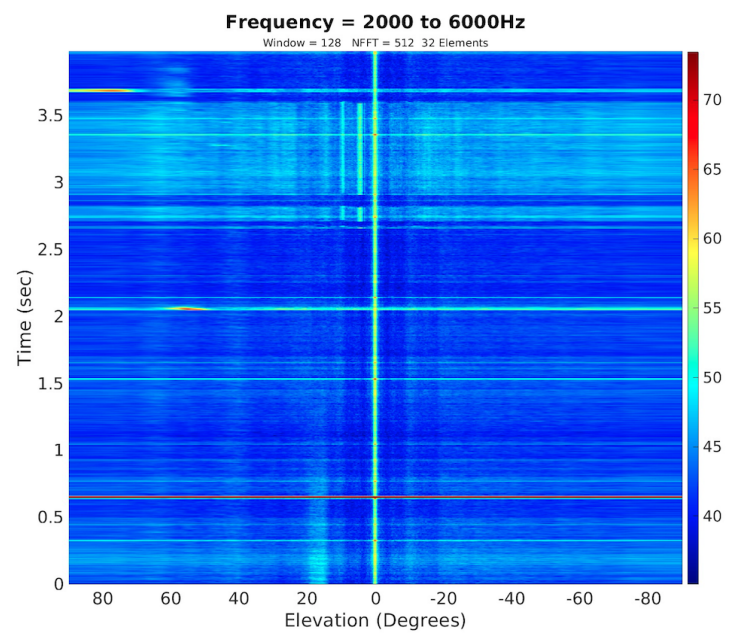

(b) Uniform weights with full DURIP array. The narrower main lobe results in a sharper $\mathrm{D} / \mathrm{E}$ for the buoy transmission between 2.8 to 3.6 seconds.

Figure 4-12: The Hanning weights on the left are swathy compared to the uniform weights, although the uniform weights result in some array overdriving showing a horizontal line across all D/Es.

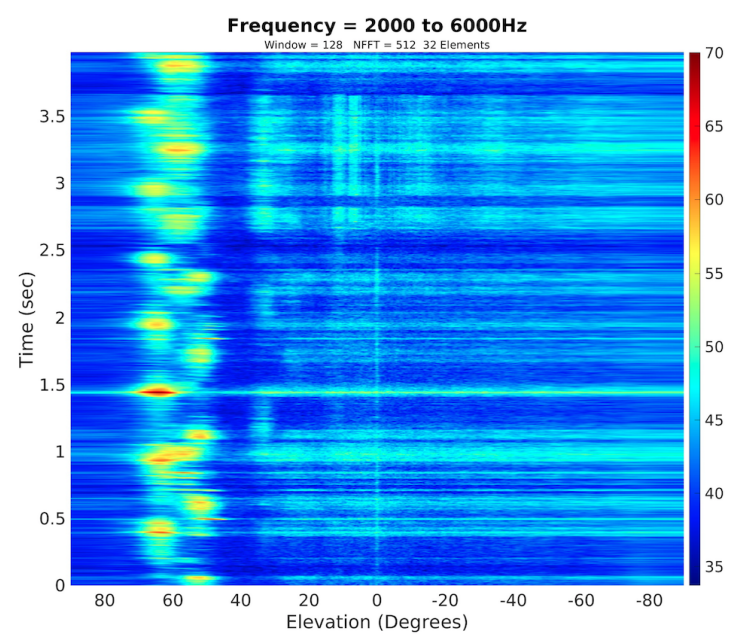

(a) Background noise is significant, but the WHOI micro-modem buoy transmission is still slightly present from 2.6 to 3.6 seconds. There are clearly two different arrival paths.

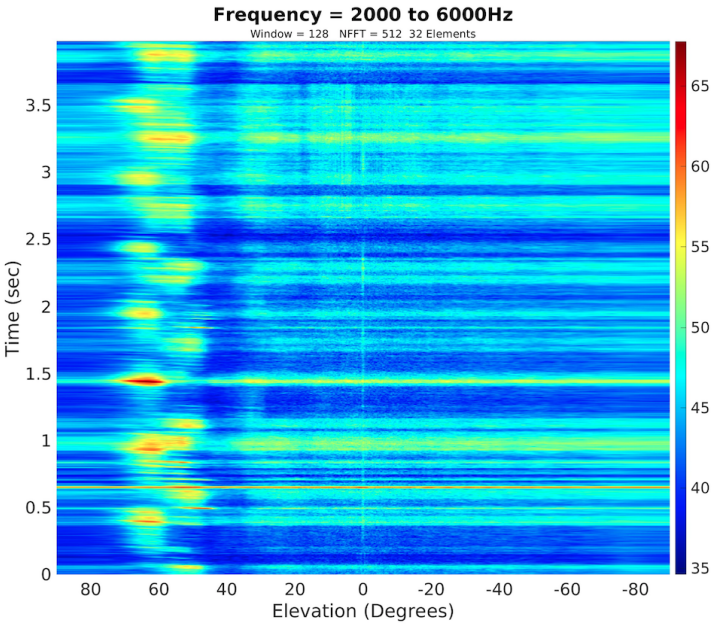

(b) Uniform weights with the full DURIP array. The impact of the side lobes and back lobe result in the transmission from the WHOI micromodem buoy being washed out by the other noise in the environment.

Figure 4-13: The Hanning weights show a micro-modem transmission that was washed out by the uniform weights. Although the uniform weighting main lobe is narrower, the additional background noise results in a washed out signal due to the stronger side and back lobes. 
noise present throughout the exercise, and is why the Hanning weights were selected to evaluate the DURIP towed array data. 


\subsection{ICEX DURIP Data Analysis}

Figure 4-14 highlights the transmissions that were missed by Macrura with black circles. The blue circles are transmissions that were received by Macrura, but there was an issue in the decoding of the message, so the message wasn't used by Macrura. These poorly demodulated transmissions led to further analysis from the communications throughout the entire ICEX that are discussed further in Chapter 5. Here I'll focus on a comparison between the black missed transmissions with the BELLHOP analysis for this area under these particular SSP conditions. Due to acoustic reciprocity, it is reasonable to assume that a similar pattern of arrivals and decoding occurred at buoys $\mathrm{H} 1, \mathrm{H} 2, \mathrm{H} 3$, and $\mathrm{H} 4$ from the Macrura transmissions.

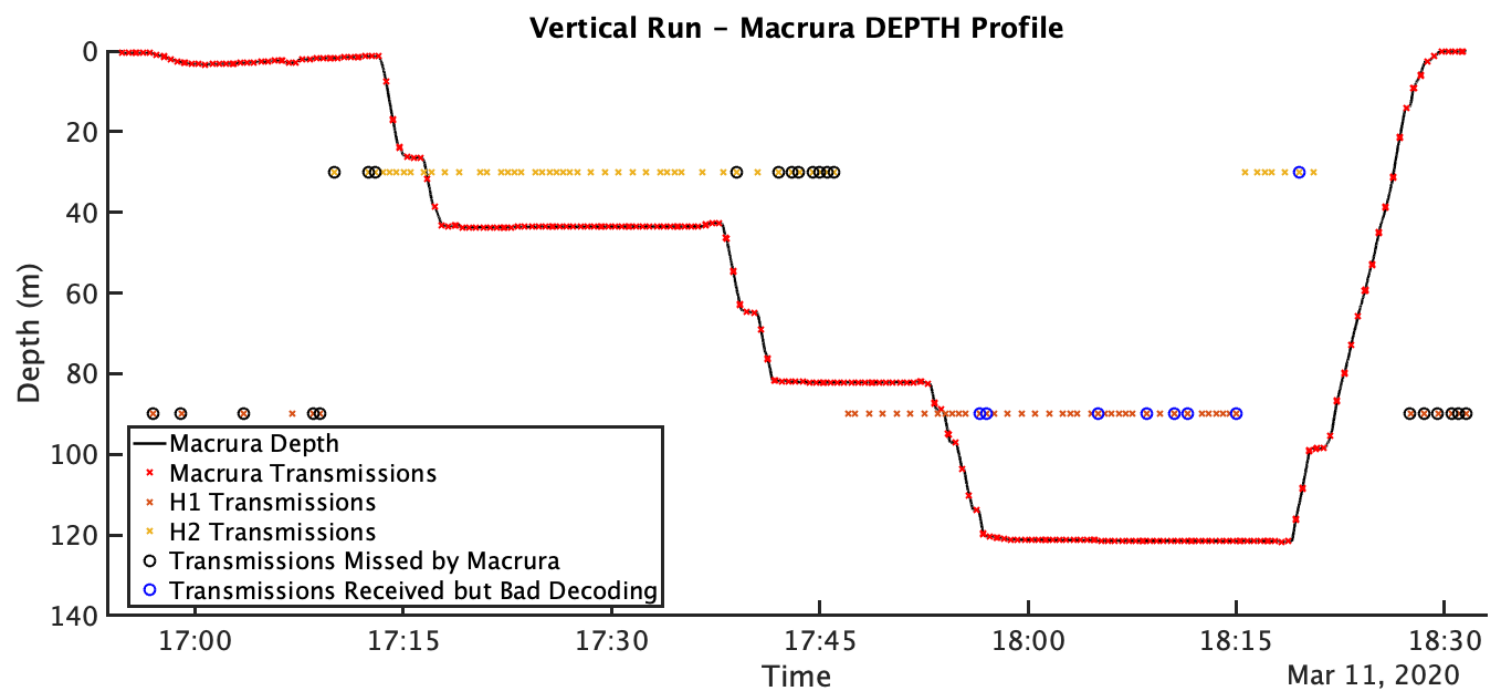

Figure 4-14: Vertical Run highlighting the transmission that were missed by Macrura.

\subsubsection{BELLHOP Projections}

Recall from Chapter 1, the dynamic SSP in the Arctic, and from Chapter 2, the BELLHOP tool for analyzing ray traces or propagation paths based on the environment. I wanted to compare a rough approximation of the XCTD with the actual XCTD cast and compare those results to the vehicle's ability to receive and decode buoy transmissions. The rough estimate of each SSP was taken from the XCTD data and extrapolated at key depths associated with HYCOM (HYbrid Coordinate Ocean Model) data. These are the depths that the Navy and other oceanographic or meteorological organizations tend to look at [30]. This was to 
evaluate if the lost fidelity from the HYCOM depths would result in a poor prediction of the likely arrival paths. Last, the objective was to compare the the BELLHOP analysis with the data received by the DURIP towed array throughout the vertical run.

This analysis was performed from the perspective of H1 (transmitting from a source depth, $z_{s}=90 \mathrm{~m}$ ) and H2 (transmitting from a source depth, $z_{s}=30 \mathrm{~m}$ ). Ray launch angles are specified from $\pm 10^{\circ}$ at $0.2^{\circ}$ increments. At these angles, based on the range to the hydrohole from $\mathrm{H} 1$ and H2, and the expected maximum array depth of $\sim 159 \mathrm{~m}$ to the center of the array. Table 4.1 shows the different depths of the vehicle and the array throughout the Vertical Run, as well as the associated color code when looking at the BELLHOP analysis plots. This was to make it easier to visualize where the array was for each segment of operations.

\begin{tabular}{||c|c|c|c|c||}
\hline \multicolumn{5}{||c||}{ Vertical Run Depths $(\mathrm{m})$} \\
\hline \hline \multirow{2}{*}{ Macrura } & \multicolumn{2}{|c|}{ DURIP Towed Array } & Array Plot \\
& Top & Center & Bottom & Color \\
\hline 0 & 23 & 38 & 53 & red \\
\hline 43.5 & 66.5 & 81.5 & 96.5 & orange \\
\hline 82 & 105 & 120 & 135 & yellow \\
\hline 121 & 144 & 159 & 174 & green \\
\hline
\end{tabular}

Table 4.1: Vertical Run Depths

NOTE: The BELLHOP analysis did NOT evaluate for bottom bounce (BB); surface to $\mathrm{BB}$; or $\mathrm{BB}$ to surface interactions. All of these trajectories with a $\mathrm{BB}$ required an initial ray launch angle in excess of $\pm 70^{\circ}$. This was unlikely due to the physical design of the transducers, their radiation pattern, and the natural tendency of higher frequency to attenuate over longer distance coupled with the surface and bottom scattering losses these ray paths required.

\section{BELLHOP Analysis for Buoy H1 : $z_{s}=90 \mathrm{~m}, r_{s}=1463 \mathrm{~m}$;} and for Buoy $\mathrm{H} 2: z_{s}=30 \mathrm{~m}, r_{s}=1635 \mathrm{~m}$

The BELLHOP analysis includes the arrivals at the other nodes as well (eg. Transmissions from $\mathrm{H} 1$ arriving at $\mathrm{H} 2, \mathrm{H} 3$, \& $\mathrm{H} 4)$. Recall that only a shallow (30 m) or deep (90 m) receiver is selected at a time. Table 4.2 breaks down the arrivals between the deep and shallow receivers by order of range from the source. Note, no transmissions from $\mathrm{H} 2$ occurred while the buoys were selected to the deep receiver. This was a conscientious team decision because 
we anticipated the surface duct would capture most noise, and just like the BELLHOP Figures 4-32 and 4-33 show the deep hydrophones are not on any of the ray paths. The deep receivers are in a shadow zone.

\begin{tabular}{|c|c|c|c|c|}
\hline \multicolumn{5}{|c|}{ Total Transmissions from $\mathrm{H} 1$ with $z_{s}=90 \mathrm{~m}$, and Receptions at buoys } \\
\hline Total H1 & Receiver & \multicolumn{3}{|c|}{$\begin{array}{c}\text { Total Receptions - Range from H1 } \\
\end{array}$} \\
\hline Transmissions & Depth $(\mathrm{m})$ & $\mathrm{H} 4(\triangle)-2045 \mathrm{~m}$ & $\mathrm{H} 2(\bigcirc)-2150 \mathrm{~m}$ & H3 $(\diamond)-3160 \mathrm{~m}$ \\
\hline 39 & 90 & 36 & 37 & 1 \\
\hline 12 & 30 & 3 & 6 & 3 \\
\hline \multicolumn{5}{|c|}{ Total Transmissions from H2 with $z_{s}=30 \mathrm{~m}$, and Receptions at buoys } \\
\hline Total H2 & Receiver & \multicolumn{3}{|c|}{ Total Receptions - Range from H2 } \\
\hline Transmissions & Depth $(\mathrm{m})$ & H3 $(\diamond)-1895 \mathrm{~m}$ & $\mathrm{H} 1(\square)-2150 \mathrm{~m}$ & $\mathrm{H} 4(\triangle)-3140 \mathrm{~m}$ \\
\hline 52 & 30 & 51 & 52 & 13 \\
\hline
\end{tabular}

Table 4.2: Transmissions from H1, $z_{s}=90 m$, and $\mathrm{H} 2, z_{s}=30 m$, received at buoys based on depth.

One particularly interesting feature present from the XCTD in Figure 4-15a is that the $z_{s}$ is near a small local sound minimum. As a result, the ray trace shows a trajectory that leaves Buoy H1, and arrives at the $90 \mathrm{~m}$ receiver on buoys $\mathrm{H} 4 \triangle, \mathrm{H} 2 \bigcirc$, \& $\mathrm{H} 3 \diamond$; in that order based on the ranges to those buoys from H1. Although different azimuthal angles are necessary to reach each buoy, the transducers are omni-directional and we're treating the SSP as spatially constant. Even though the XCTD shows that ray path is possible, it seems highly improbabe compared to the remaining other ray traces for $\mathrm{H} 1, z_{s}=90 \mathrm{~m}$. Interestingly though, during the vertical run, out of 39 transmissions from H1, H3 only received 1 at its $90 \mathrm{~m}$ hydrophone. This stat is more in-line with the checks more with the of those transmissions.

Although the small sound minimum was present when the XCTD cast was taken, when doing the BELLHOP analysis, because that sound minimum also correlates with the source depth, the shallow ray trajectories, near $0^{\circ}$, are dominated by this minimum and propagate straight for a long period. This is unrealistic as noted from the actual arrivals at buoy H3, and highlights the limitations and risks of using the raw data, and assuming the SSP is spatially constant. This minimum is not present at the 2100 XCTD cast and is lost when the HYCOM estimate of the SSP is taken. 

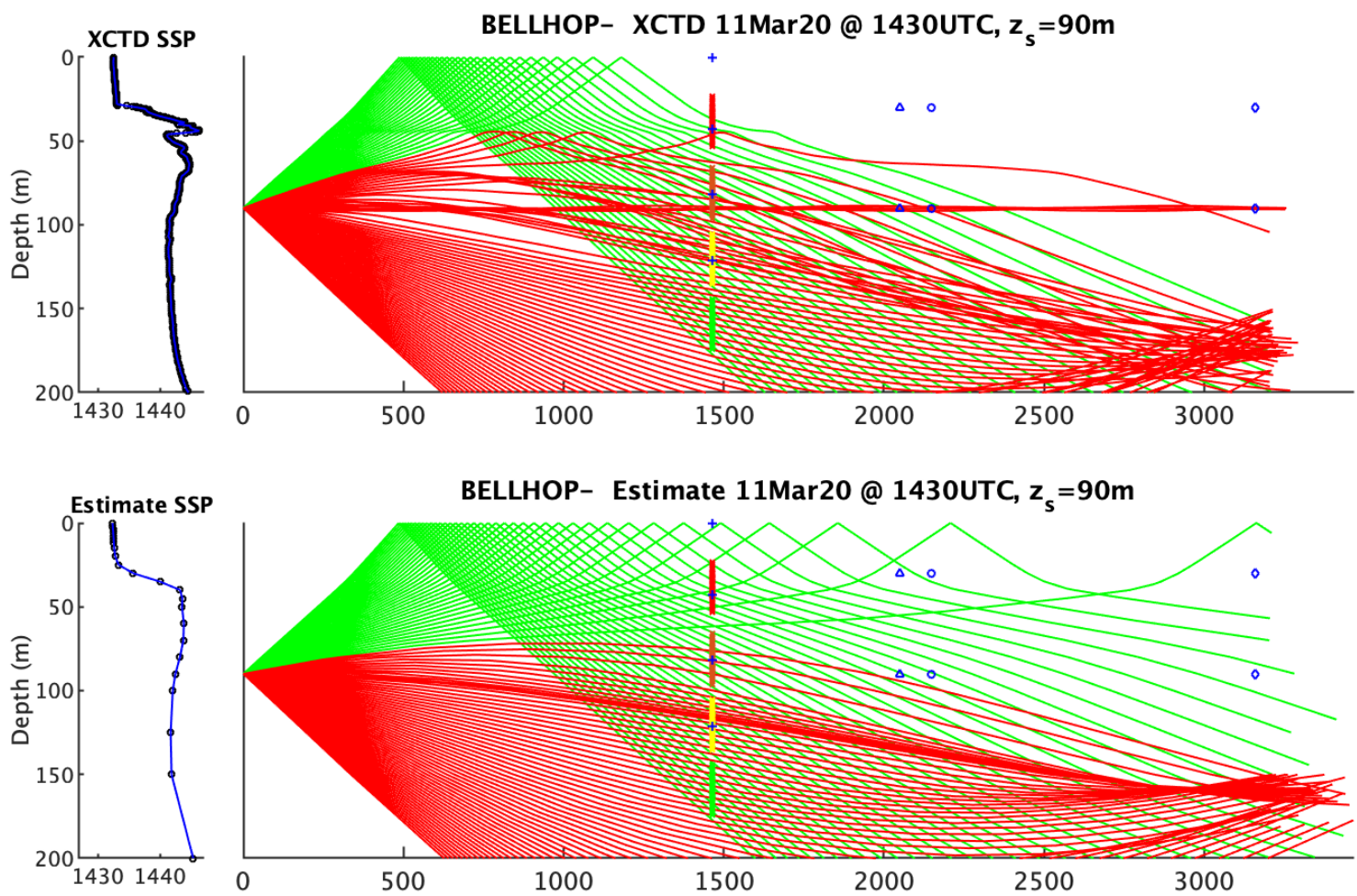

(a) Ray trace of XCTD on 03-11-2020 at 1430UTC raw vs. HYCOM estimate
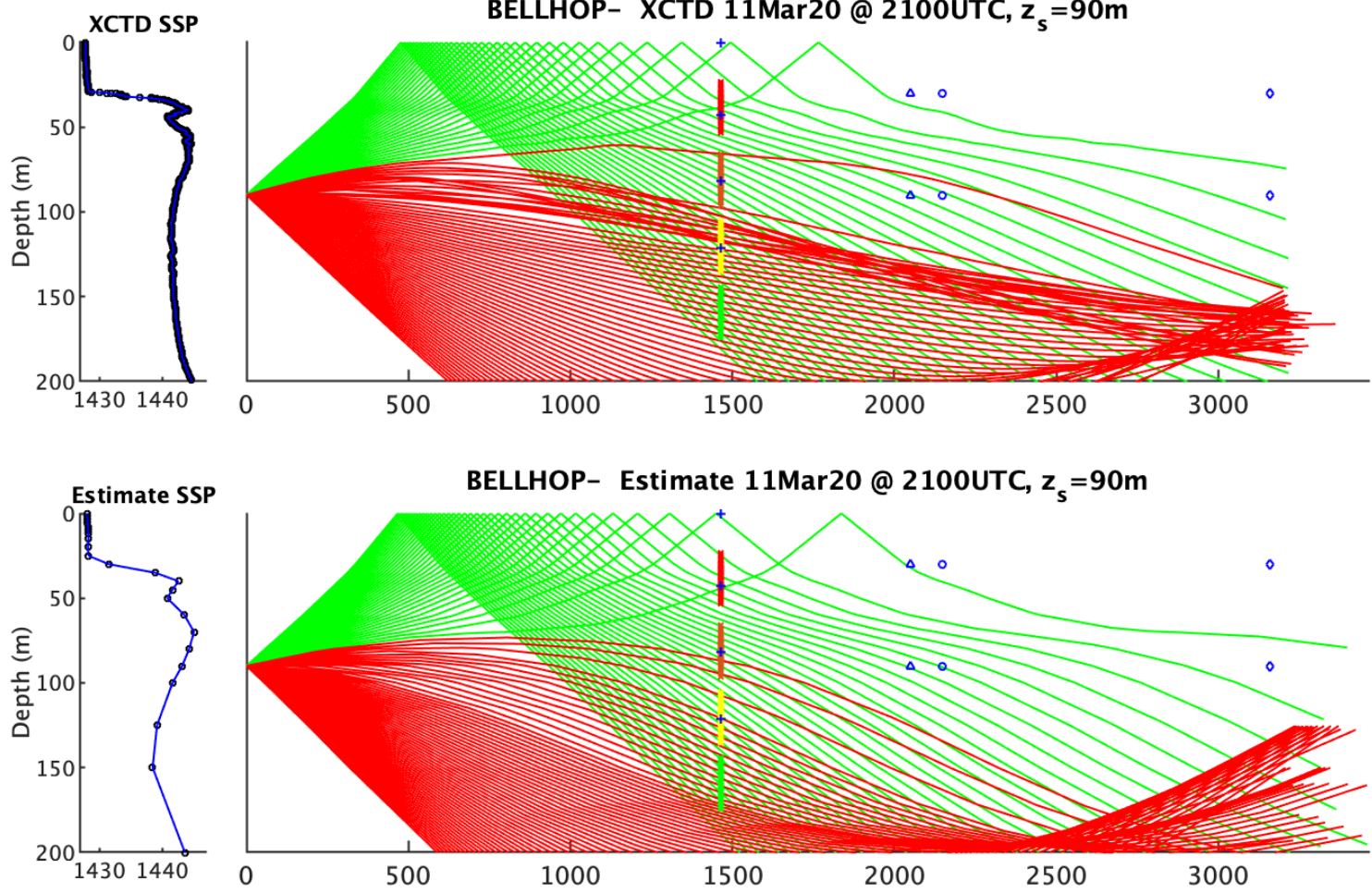

(b) Ray trace of XCTD on 03-11-2020 at 2100UTC raw vs. HYCOM estimate

Figure 4-15: Ray traces from 1430UTC and 2100UTC with $z_{s}=90 \mathrm{~m}$ 

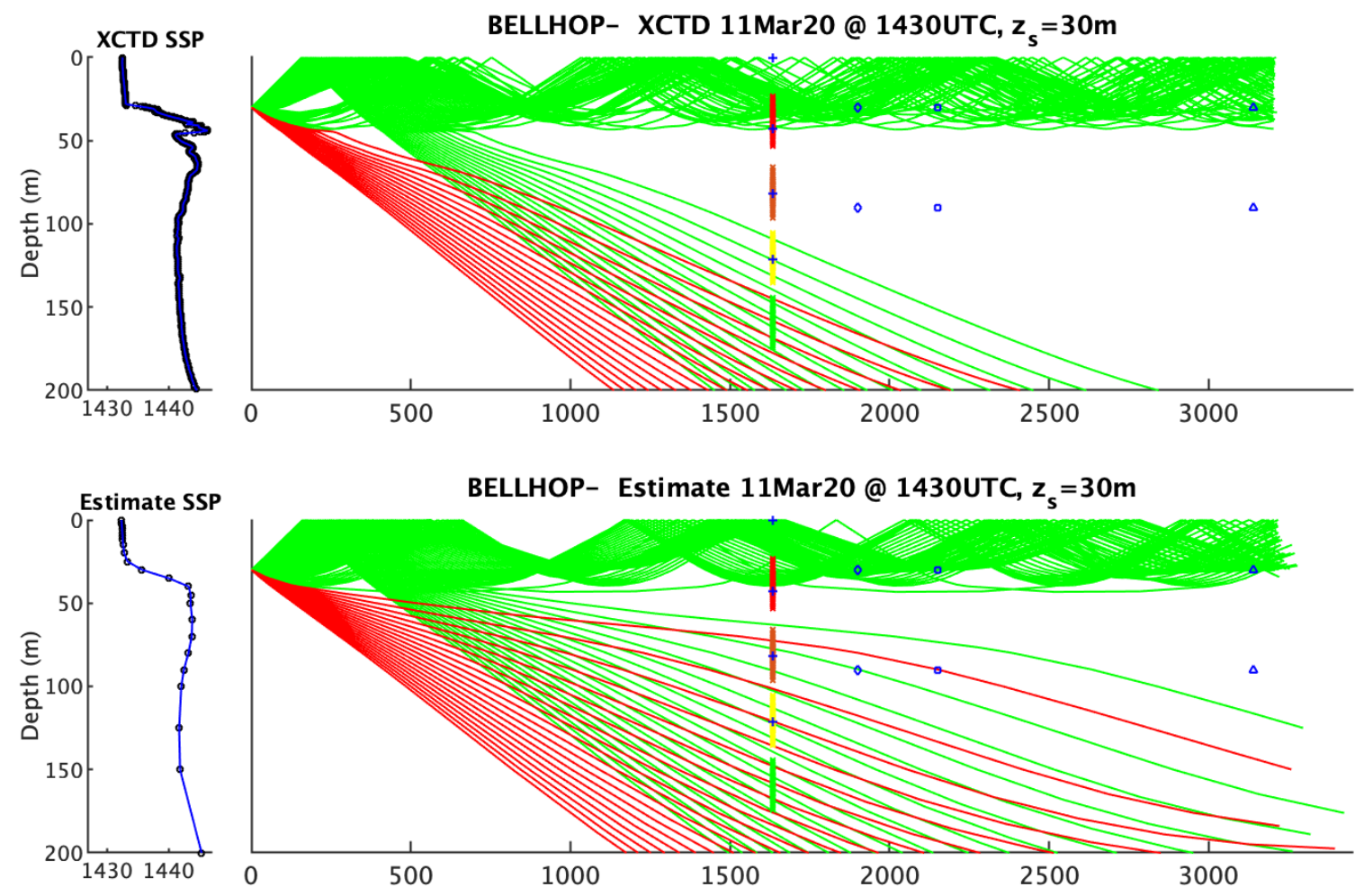

(a) Ray trace of XCTD on 03-11-2020 at 1430UTC raw vs. HYCOM estimate
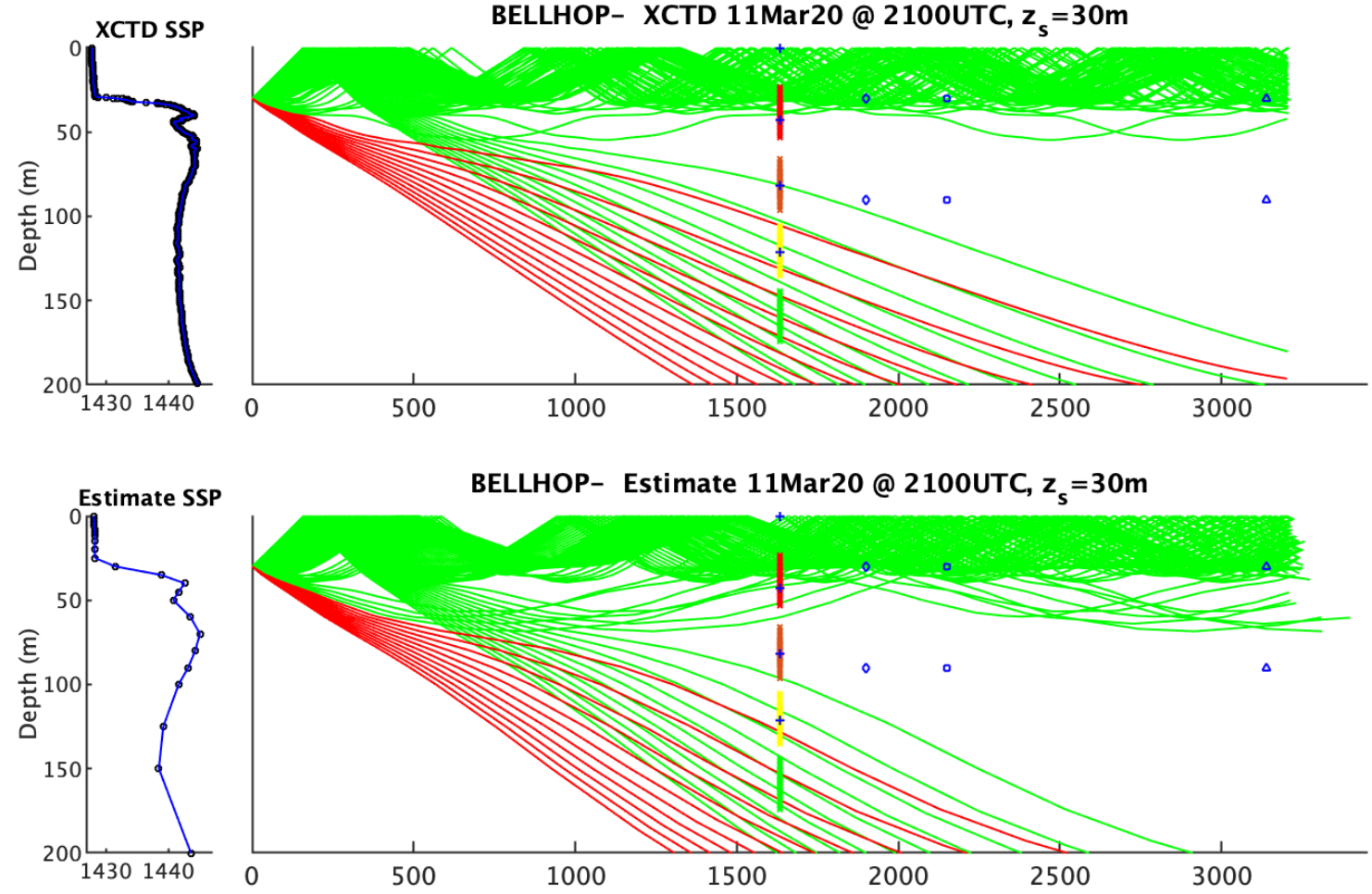

(b) Ray trace of XCTD on 03-11-2020 at 2100UTC raw vs. HYCOM estimate

Figure 4-16: Ray traces from 1430UTC and 2100UTC with $z_{s}=30 \mathrm{~m}$ 


\subsubsection{DURIP Array Vertical Run Beamforming}

The Vertical Run occurred between 1655 to 1830 UTC on 03-11-2020 and is plotted in Figure 1-11. Here I'll highlight a few transmissions from the shallow buoy $(30 \mathrm{~m})$ and deep buoy (90 m) while the DURIP towed array is at each depth interval from Table 4.1. Of all the transmission that occurred during the Vertical Run, these were the best looking transmissions for each depth. Figure 4-17 highlights the beamformed DURIP towed array data that will be presented. Each transmission is highlighted with a blue circle and numbered. Those numbers correlate with the "Transmission \#" that are in the subsequent figures.

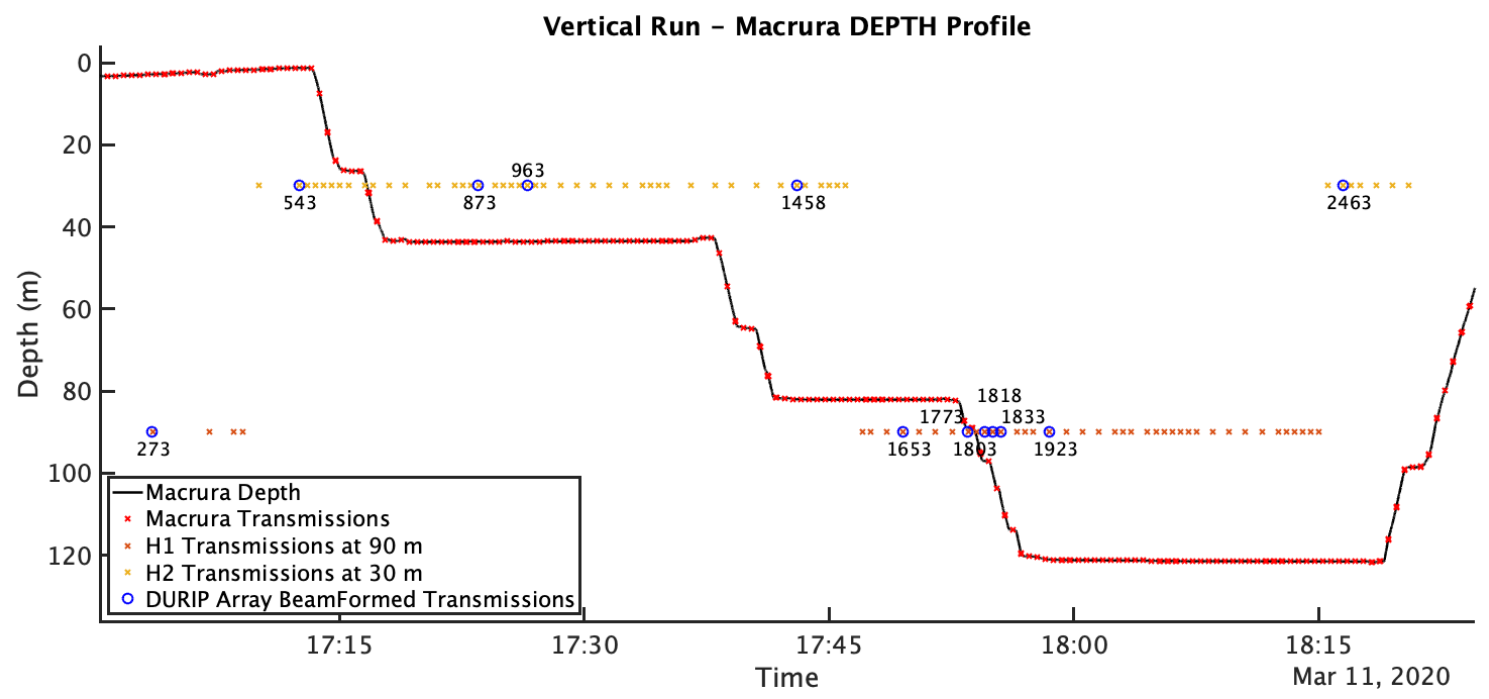

Figure 4-17: Vertical Run highlighting the transmissions that were captured at each depth. 


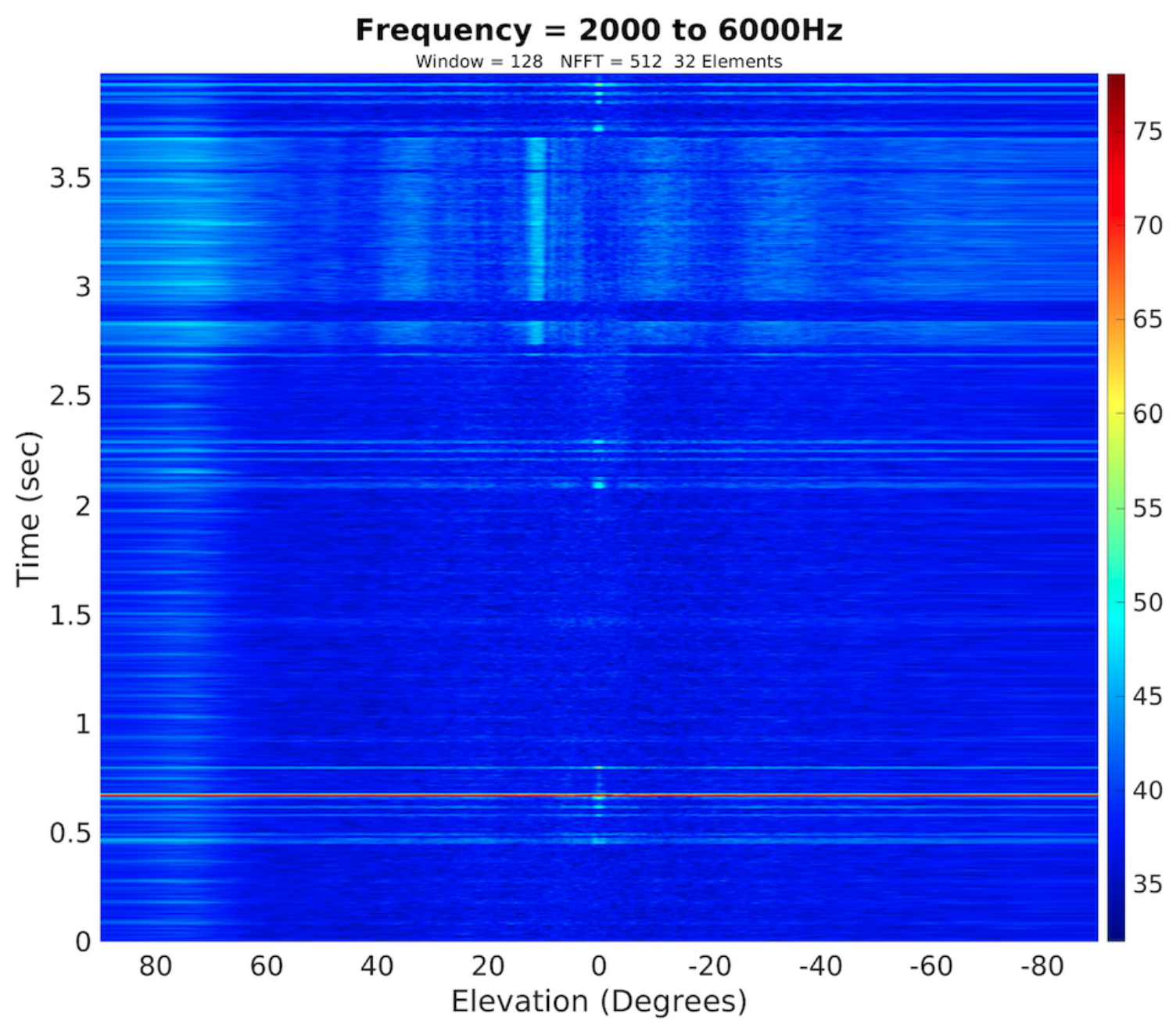

Figure 4-18: Transmission \#273. $z_{s}=\mathrm{H} 190 \mathrm{~m}$. Array $\sim 38 \mathrm{~m}$. Only a single arrival is clearly visible at roughly $+11.2^{\circ}$. The swathy nature checks with the variability seen in the water column from Figures 4-32 and 4-33. 


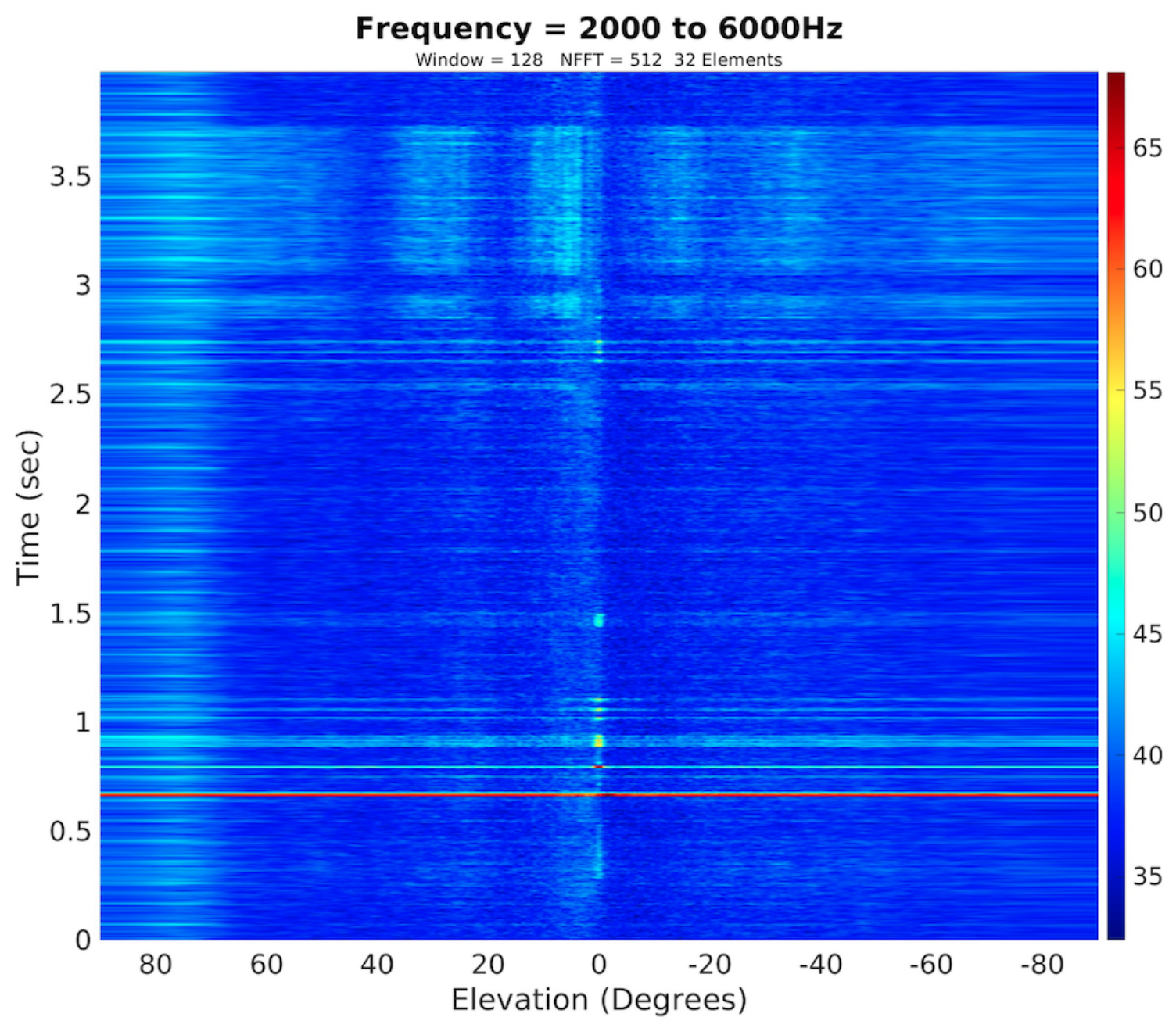

Figure 4-19: Transmission \#543. $z_{s}=\mathrm{H} 230 \mathrm{~m}$. Array $\sim 38 \mathrm{~m}$. 


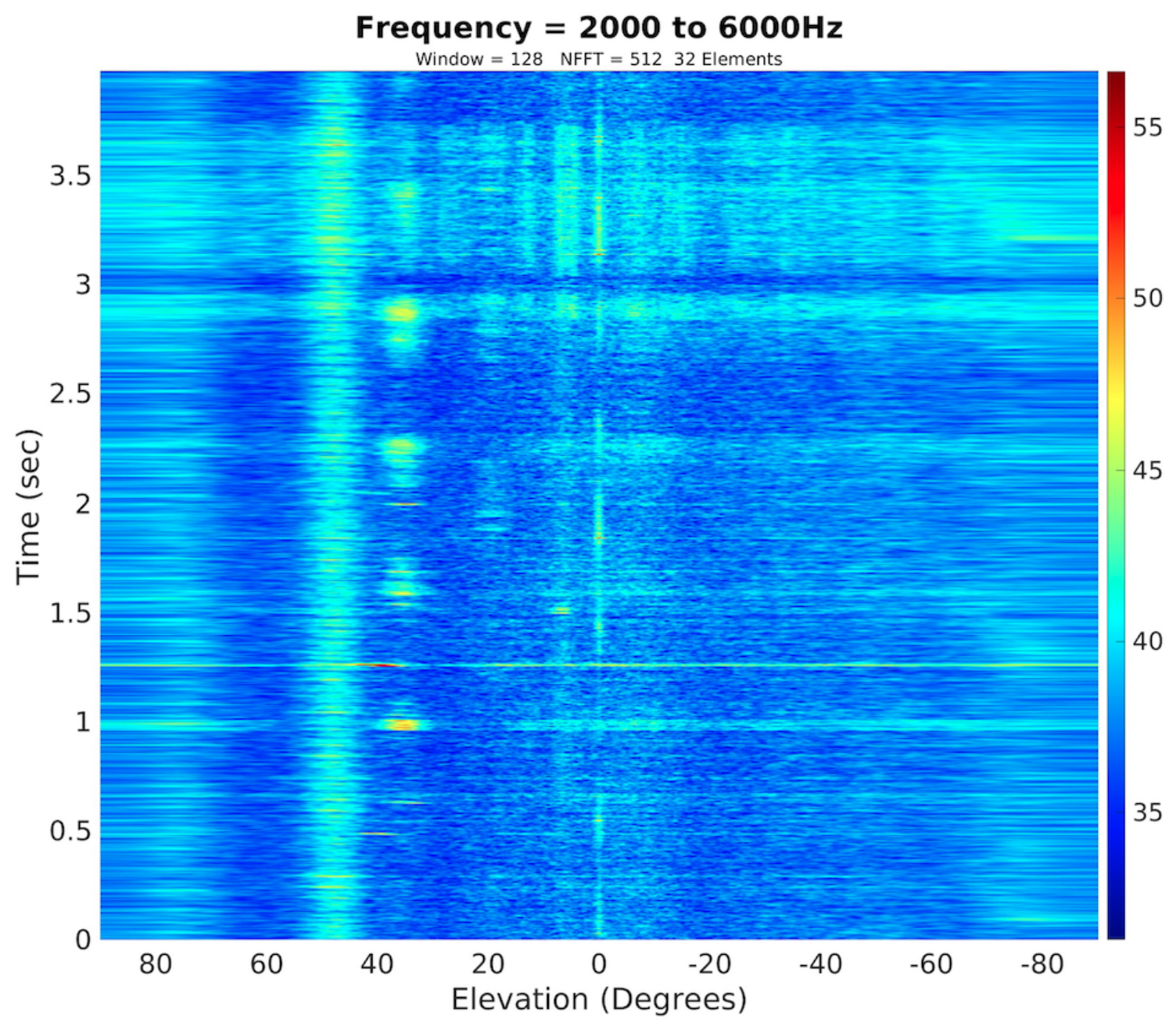

Figure 4-20: Transmission \#873. $z_{s}=\mathrm{H} 230 \mathrm{~m}$. Array $\sim 82 \mathrm{~m}$. Transmission was captured in the shadow zone. It's slight but the transmission appears swathy between $+4^{\circ}$ and $+8^{\circ}$. It's possible it could be two separate arrival paths, but there isn't a clear separation between the two arrival angles. There is also a possible arrival at $+13^{\circ}$, although this seems unlikely based on Figures 4-32 and 4-33. 


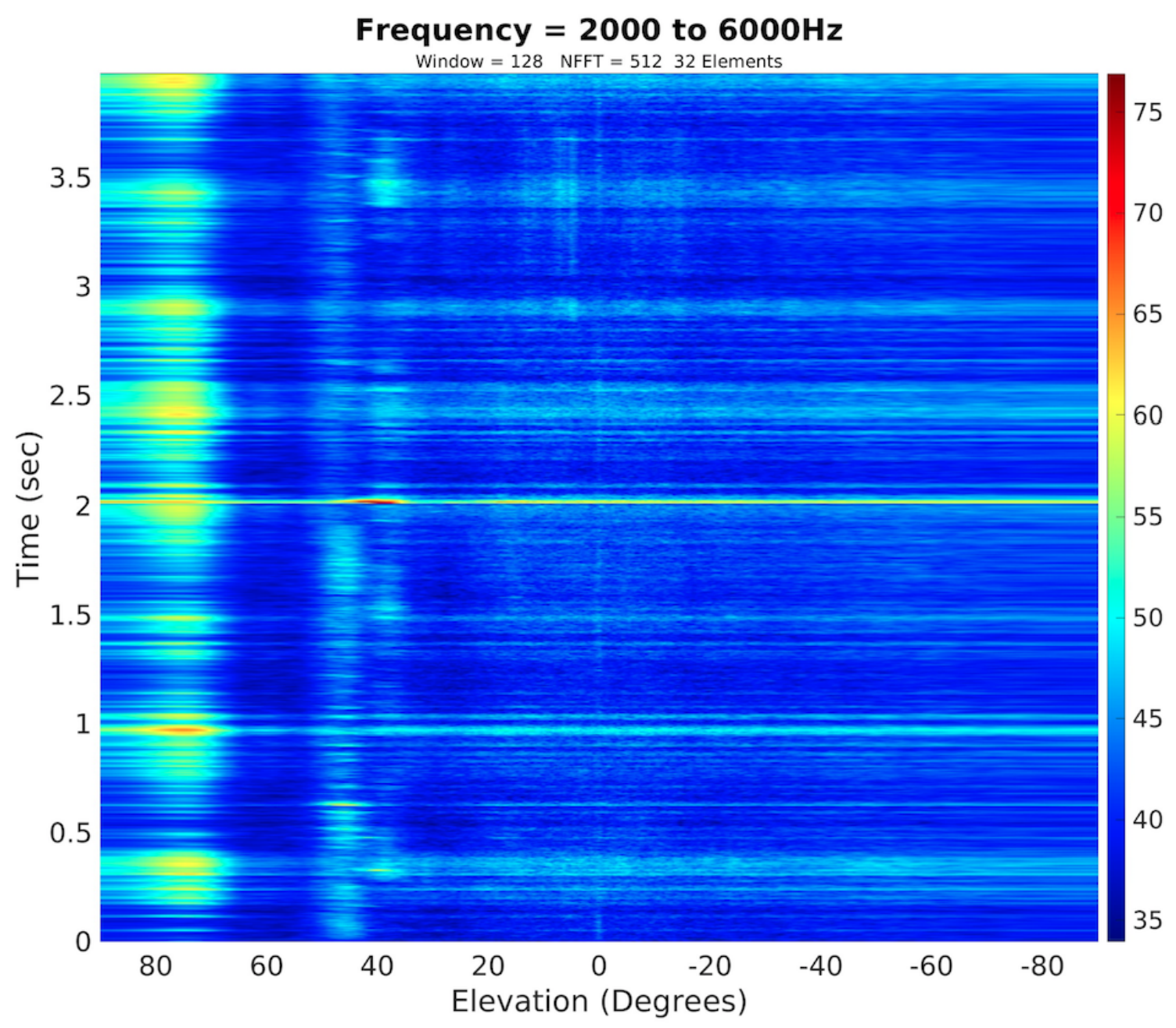

Figure 4-21: Transmission \#963. $z_{s}=\mathrm{H} 230 \mathrm{~m}$. Array $\sim 82 \mathrm{~m}$. Transmission was captured in the shadow zone. It's slight but the transmission appears lightly between $+4^{\circ}$ and $+8^{\circ}$. It's possible it could be two separate arrival paths, but it's so weak it's hard to tell. 


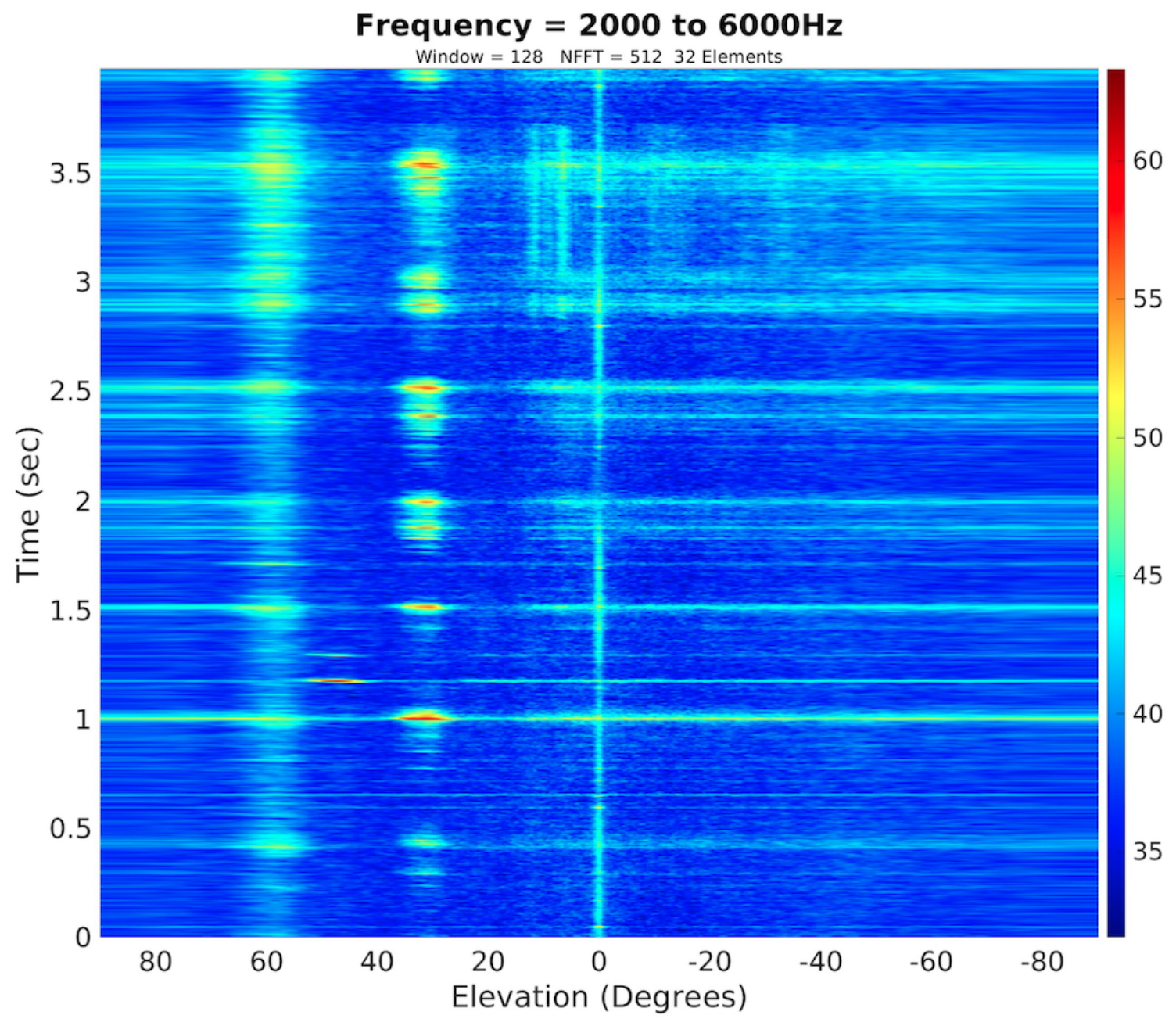

Figure 4-22: Transmission \#1458. $z_{s}=\mathrm{H} 230 \mathrm{~m}$. Array $\sim 120 \mathrm{~m}$. 


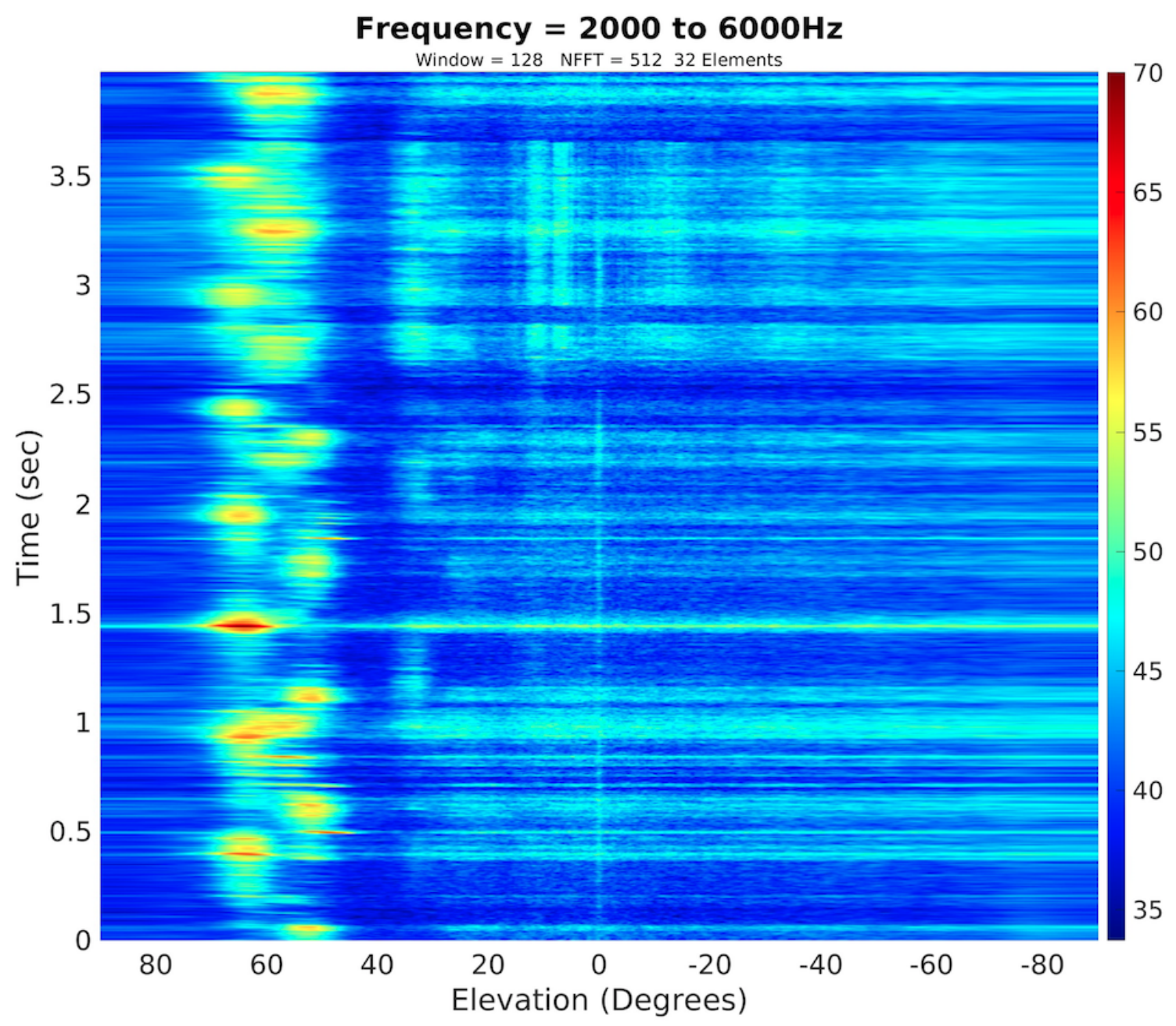

Figure 4-23: Transmission \#1653. $z_{s}=\mathrm{H} 1 \quad 90 \mathrm{~m}$. Array $\sim 120 \mathrm{~m}$. A slight time delay is present between the $+6.9^{\circ}$ and $+11.3^{\circ}$ arrivals. Both arrivals are broad and diffuse or "swathy." 


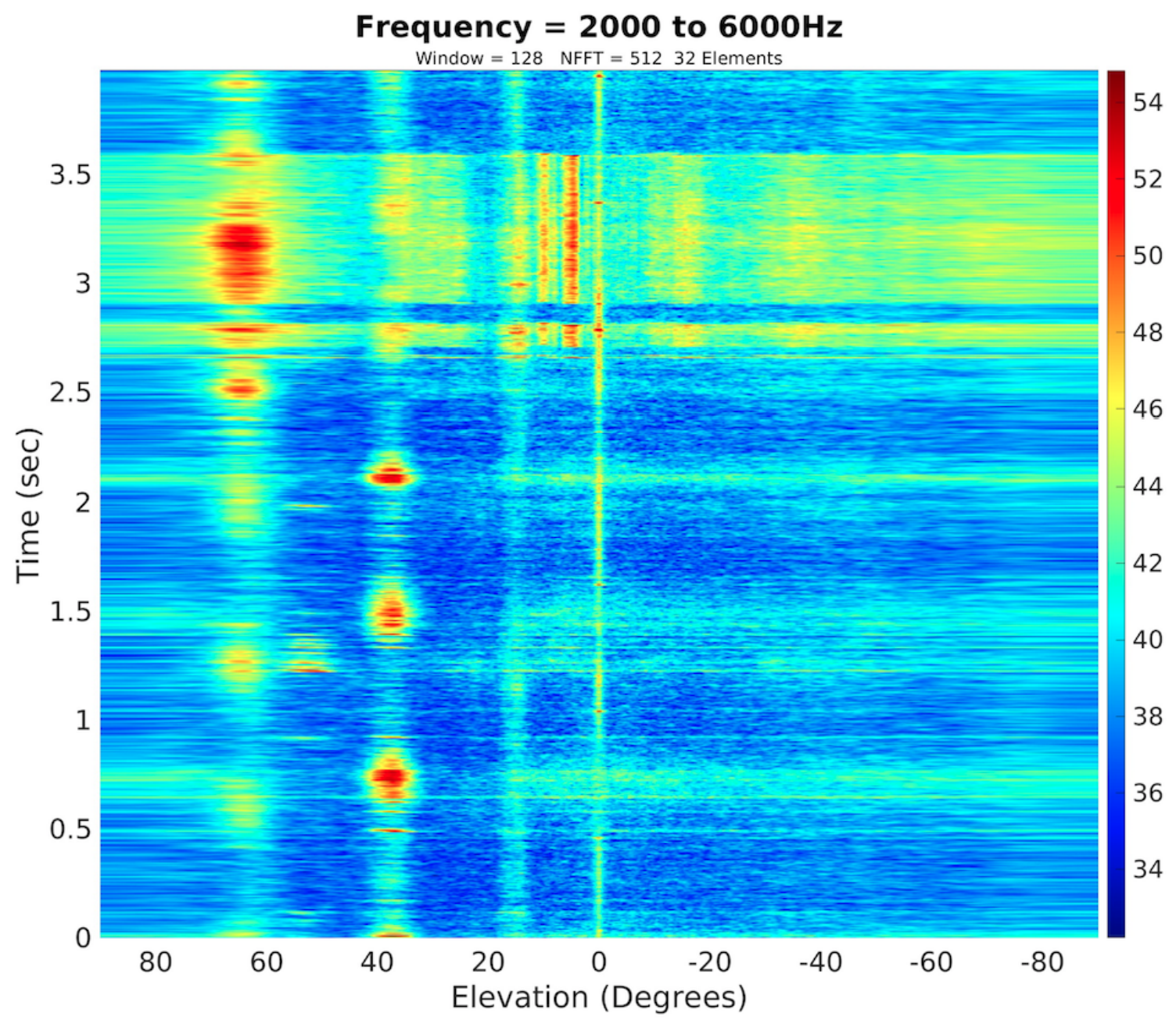

Figure 4-24: Transmission \#1773. $z_{s}=\mathrm{H} 1 \quad 90 \mathrm{~m}$. Array $\sim 127 \mathrm{~m}$. A slight time delay is present between the $+4.9^{\circ}$ and $+10^{\circ}$ arrivals. Transmission occurred during the final vehicle depth excursion from 82 to $121 \mathrm{~m}$. The direct path arrival is sharp and crisp, easily discernible even with the other interfering background noise. 


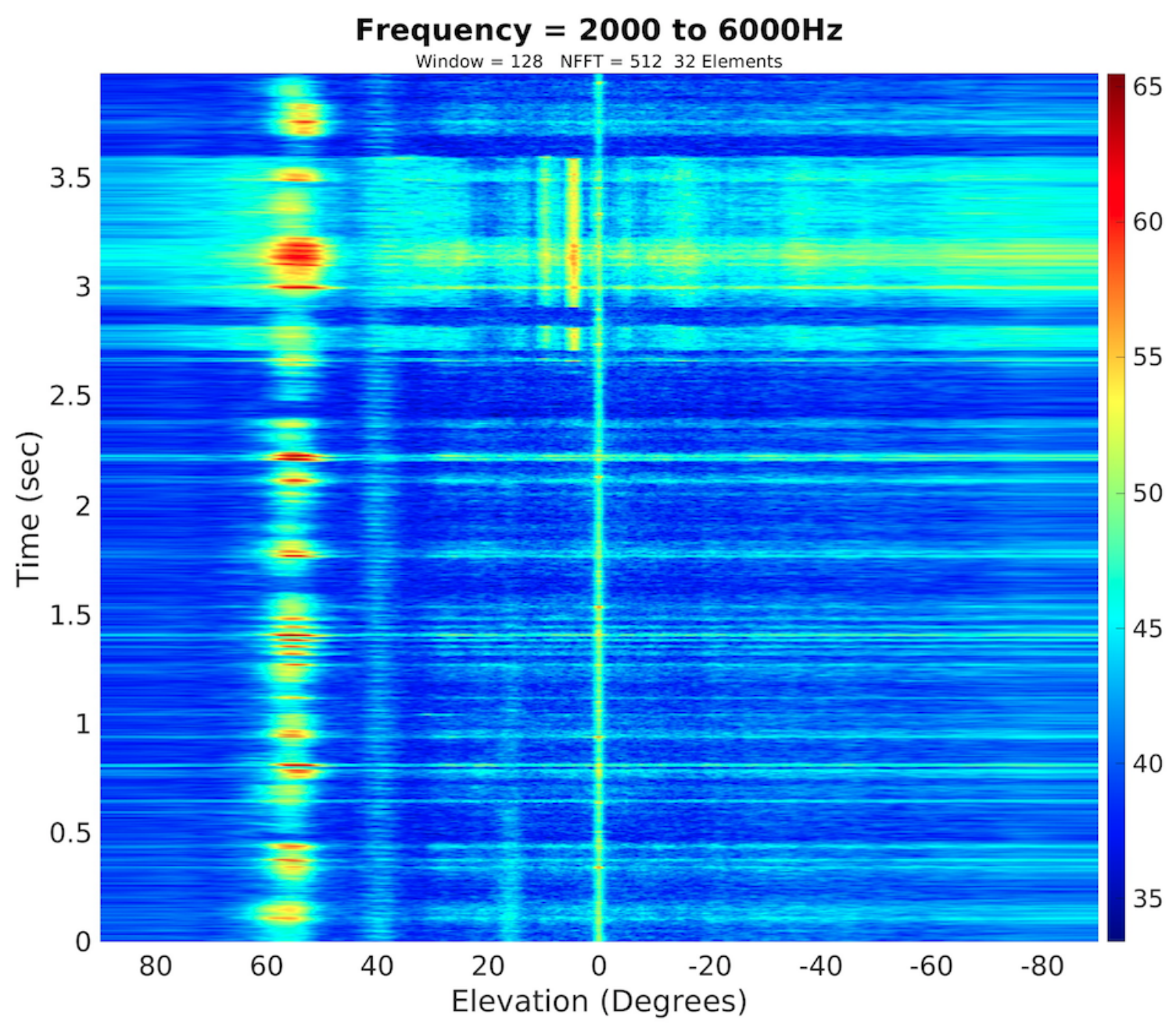

Figure 4-25: Transmission \#1803. $z_{s}=\mathrm{H} 190 \mathrm{~m}$. Array $\sim 135 \mathrm{~m}$. Transmission occurred during the final vehicle depth excursion from 82 to $121 \mathrm{~m}$. The direct path arrival is sharp and crisp, easily discernible. 


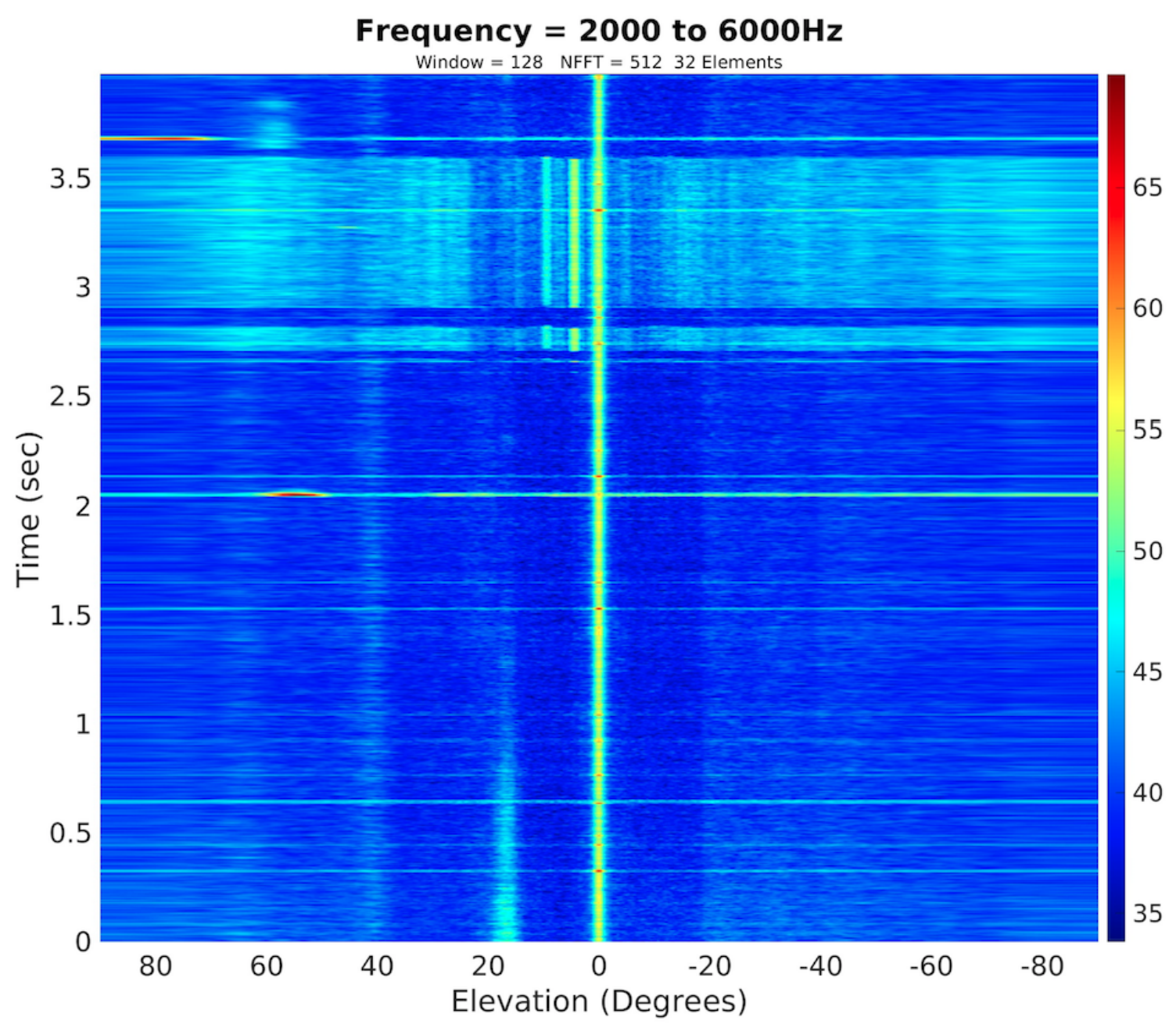

Figure 4-26: Transmission \#1818. $z_{s}=\mathrm{H} 1 \quad 90 \mathrm{~m}$. Array $\sim 139 \mathrm{~m}$. A slight time delay is present between the $+4.4^{\circ}$ and $+9.5^{\circ}$ arrivals. Transmission occurred during the final vehicle depth excursion from 82 to $121 \mathrm{~m}$. The direct path arrival is sharp and crisp, easily discernible. 


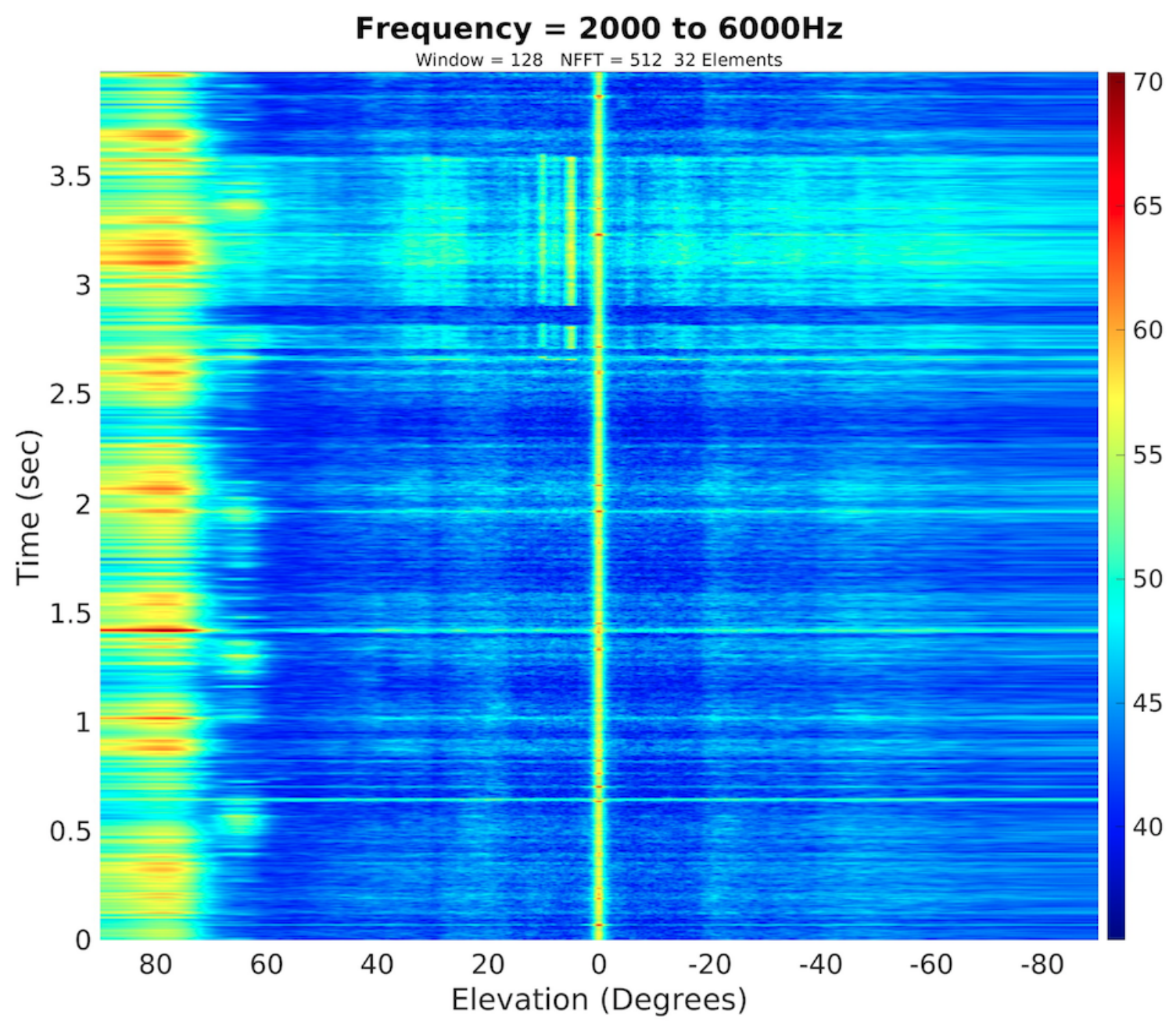

Figure 4-27: Transmission \#1833. $z_{s}=\mathrm{H} 1 \quad 90 \mathrm{~m}$. Array $\sim 145 \mathrm{~m}$. A slight time delay is present between the $+5^{\circ}$ and $+10.2^{\circ}$ arrivals. Transmission occurred during the final vehicle depth excursion from 82 to $121 \mathrm{~m}$. The direct path arrival is sharp and crisp, easily discernible even with the background noise in the upper D/Es. 


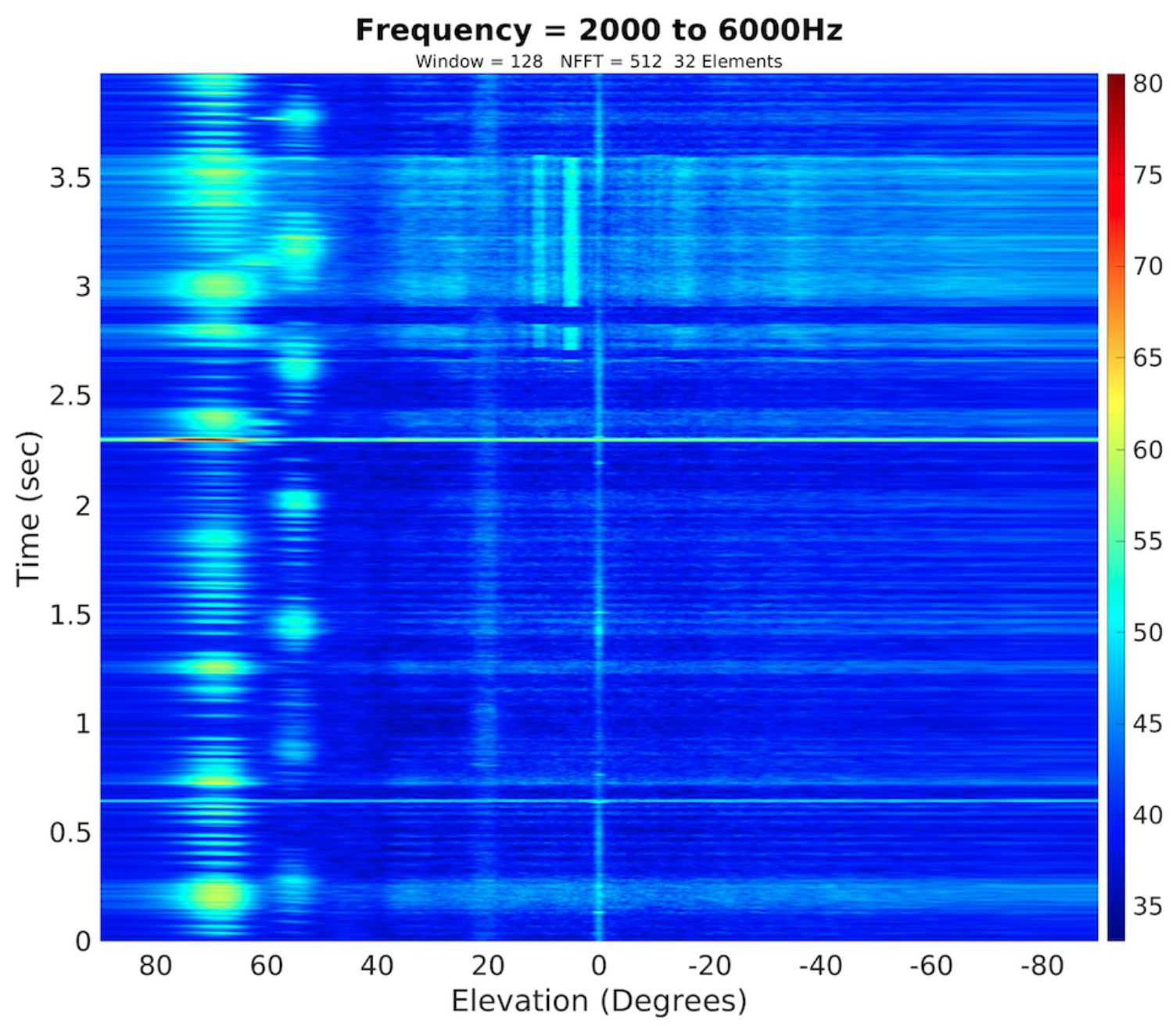

Figure 4-28: Transmission \#1923. $z_{s}=\mathrm{H} 1 \quad 90 \mathrm{~m}$. Array $\sim 159 \mathrm{~m}$. A slight time delay is present between the $+5.1^{\circ}$ and $+10.9^{\circ}$ arrivals. Although not as sharp as \#1833, it's quite cleaner than \#1653 with a similar amount of background noise. Would decode more consistently than \#1653. 


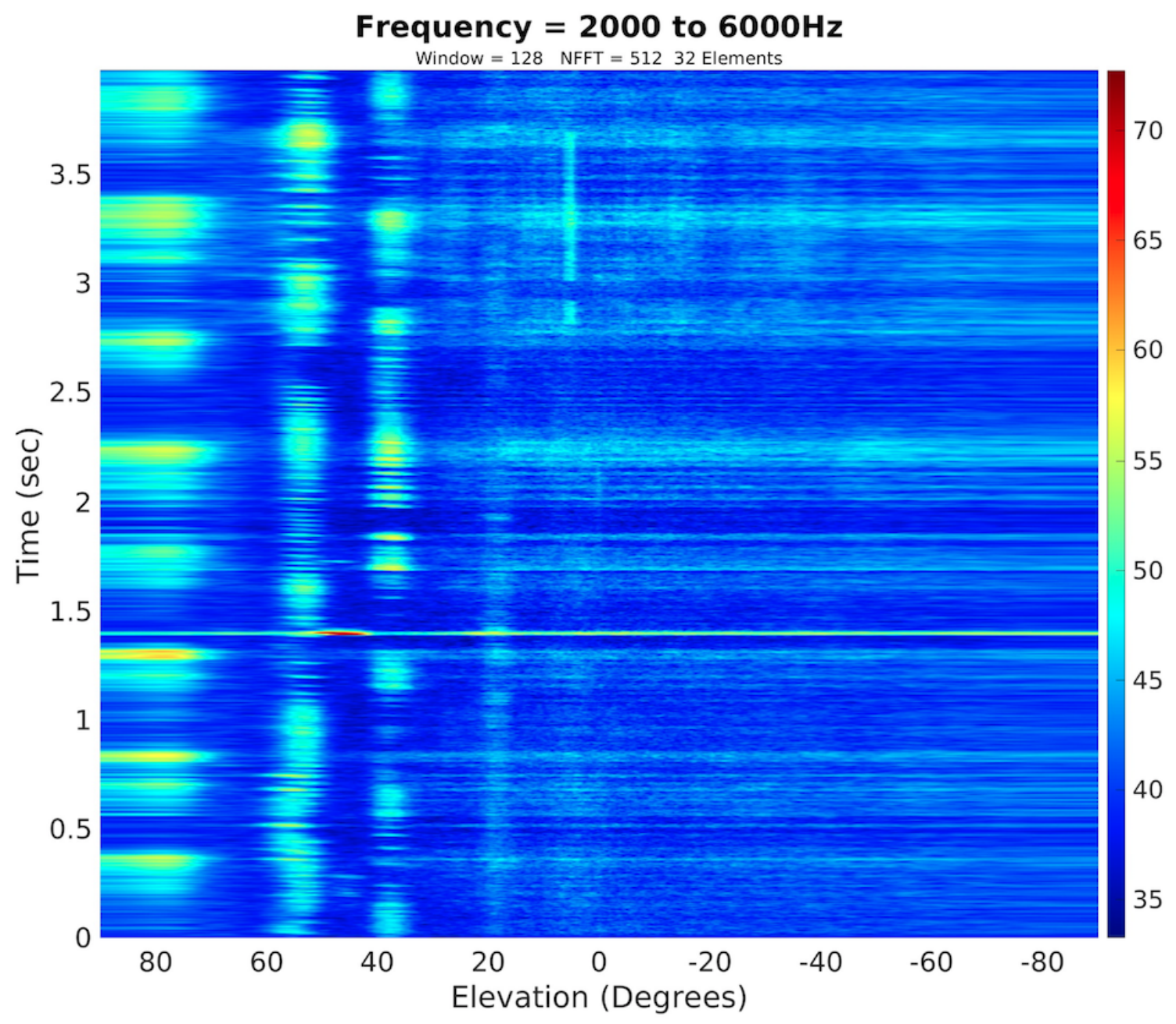

Figure 4-29: Transmission \#2463. $z_{s}=\mathrm{H} 230 \mathrm{~m}$. Array $\sim 159 \mathrm{~m}$. Only a single arrival path is visible at $+5.2^{\circ}$. Potentially due to the close arrival angle for both a direct path and surface reflected transmission seen in Figure 4-32 or the BELLHOP ray traces in Figures 4-16a, and 4-16b. Both arrivals appear to merge and are indistinguishable. 
A few of these figures actually do a great job showing both the different arrival paths that occur, but even show the slight time delay that occurs from the different trajectory arriving at the DURIP towed array. I think a few of the transmissions that this subtle time delay is most easily recognizable on are transmission \#1653, 1773, 1818, 1833, and 1923 (Figures 4-23, 4-24, 4-26, 4-27, and 4-28). This slight delay is the result of the ray trajectories we discussed in Figure 4-2 and are visible in Figure 4-15. Even at such a short range (roughly $1500 \mathrm{~m}$ from H1 to Macrura) and these high sound speeds $(\approx 1440 \mathrm{~m} / \mathrm{s})$, the different propagation paths these acoustic waves take to arrive at the DURIP towed array results in a small visible time delay. The arrival path nearest the beam is direct path; typically red in the ray trace Figures 4-15 and 4-16. The steeper angle transmission shows up with a small time delay after the direct path signal already arrived, colored green in the ray trace figures. This signal is the part of the acoustic wave that went upward and bounced off the ice and came back down to the array.

\subsection{Arrival Angle Analysis}

The objective was to compare the ray trace approximations with the actual vertical DURIP towed array data. Figures 4-30 and 4-31 compare the XCTDs and the HYCOM approximations for a 90 m source, and Figures 4-32 and 4-33 compare the XCTDs and the HYCOM approximations for a $30 \mathrm{~m}$ source. These figures are a culmination of all the data streams into a single plot.

The arrival angle calculations were made using BELLHOP and the signal amplitude to define how large the circles are. They're compared to the arrival angles from the DURIP towed array. The DURIP towed array data is the same for both $30 \mathrm{~m}$ and $90 \mathrm{~m}$ figures since it represents all of the $30 \mathrm{~m}$ or $90 \mathrm{~m}$ transmissions that occurred with the array at that particular depth. Recall from Figure 1-8 that the DURIP array is $30 \mathrm{~m}$ in length. However for these plots, the depth selection for the array data was based on the center of the towed array while it was vertical throughout this experiment, see Table 4.1.

The array is shown connected to the vehicle on the right with the center of the array highlighted with a blue circle at each depth that transmission data was used to build the blue DURIP array data set from. Most of the time the vehicle was changing depth, the array data was poor with the exception of the last depth shift from 82 to $121 \mathrm{~m}$. During this 
depth excursion the DURIP towed array data was good and transmission \#1773, 1803, 1818, and 1833 were from that depth excursion (Figures 4-24, 4-25, 4-26, and 4-27). Lastly, the black circles are plotted at depths when transmissions occurred and convey the transmissions decoded by Macrura at that depth.

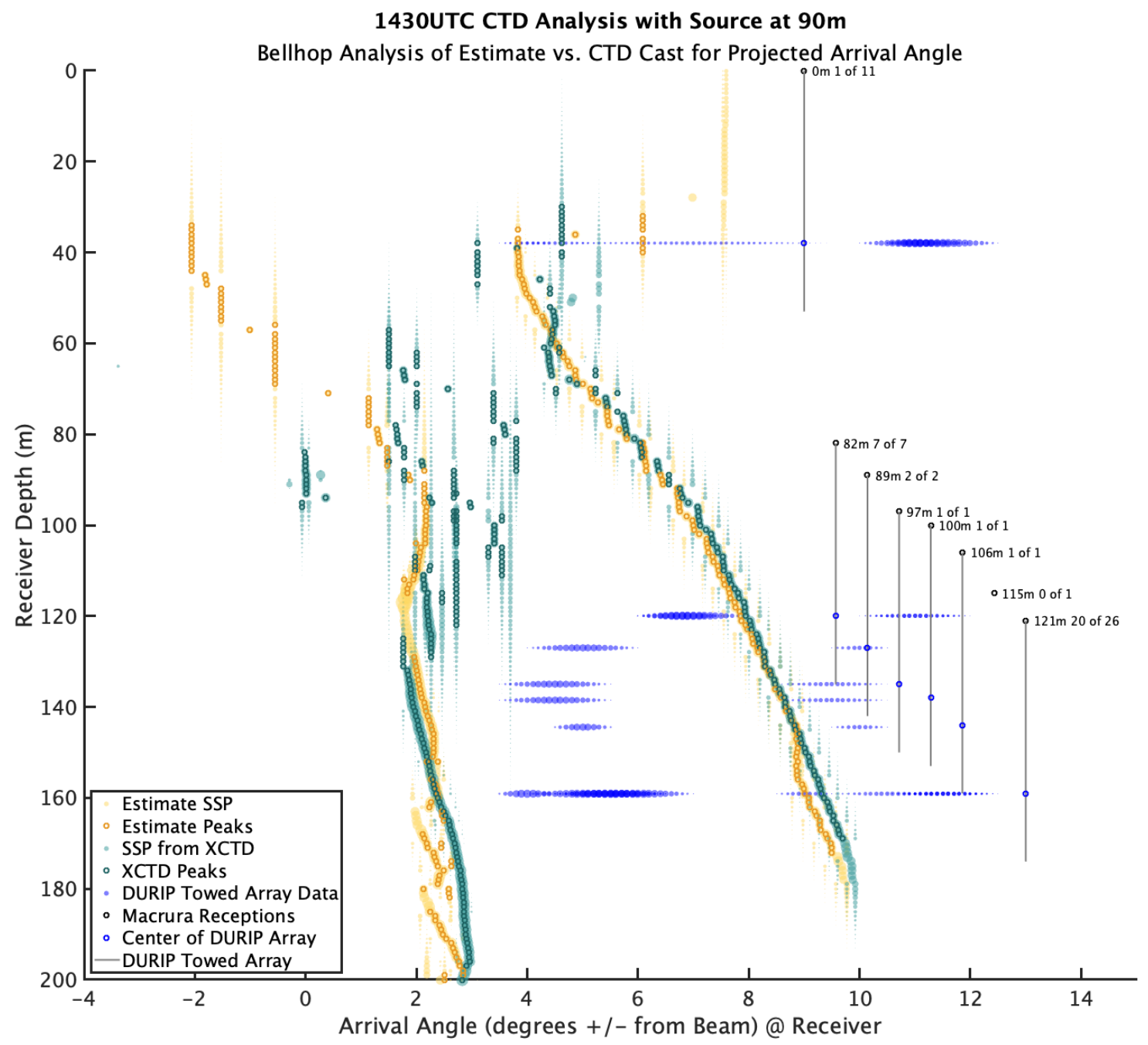

Figure 4-30: Expected arrival angle from the XCTD from 1430 vs. the HYCOM approximation for the SSP for a $90 \mathrm{~m}$ source depth. DURIP towed array data was used to determine the arrival angle from all $90 \mathrm{~m}$ transmissions at that array depth. As well, the vehicle's statics for receptions from $\mathrm{H} 1\left(z_{s}=90 \mathrm{~m}\right)$ are included.

\subsubsection{H1 Analysis with Source Depth, $z_{s},=90 \mathrm{~m}$}

The plots show how similar the HYCOM approximation and the XCTD are to one another. In Figure 4-31, the HYCOM approximation diverges from the XCTD between 110-170 m 


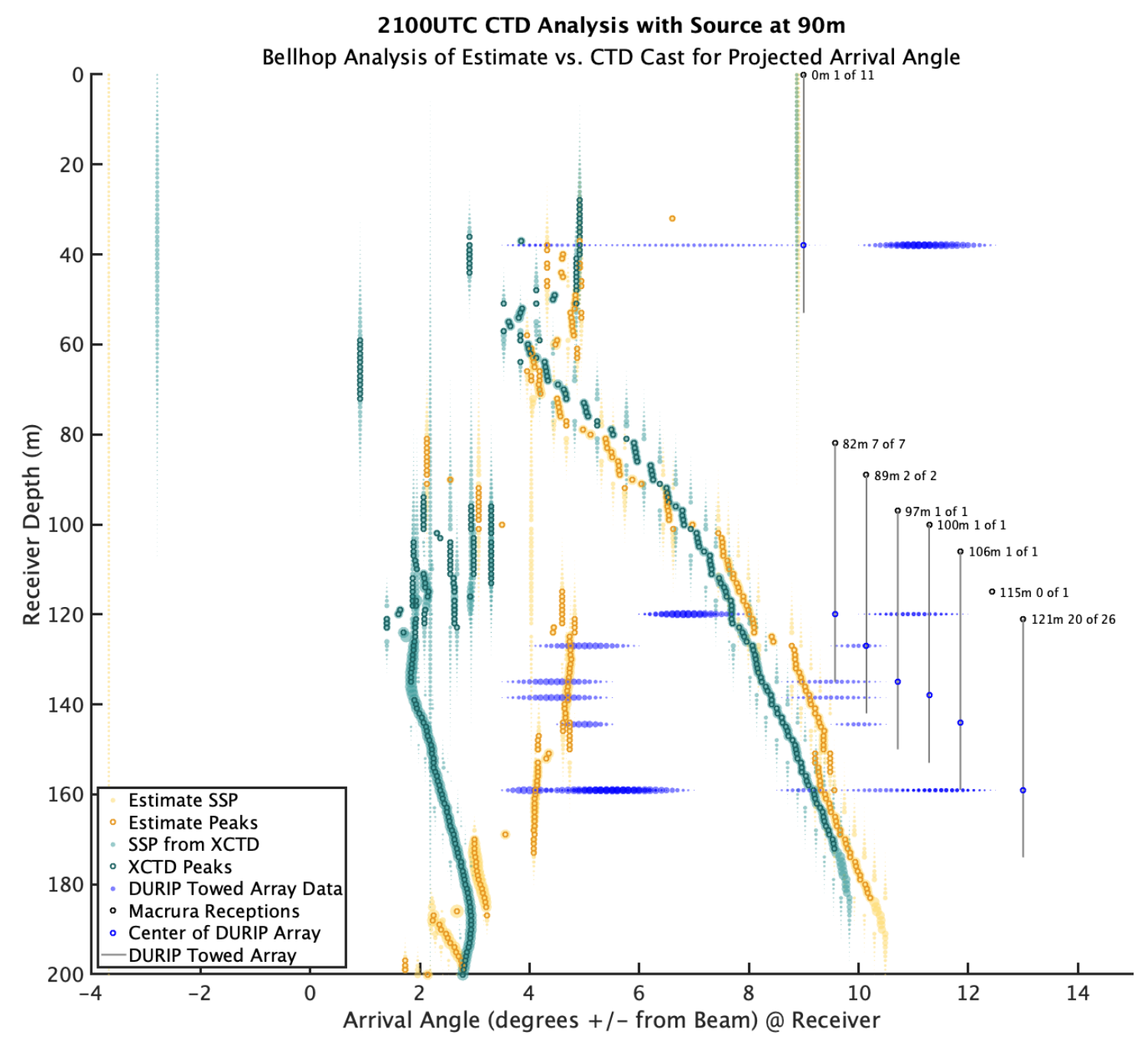

Figure 4-31: Expected arrival angle from the XCTD from 2100 vs. the HYCOM approximation for the SSP for a $90 \mathrm{~m}$ source depth. DURIP towed array data was used to determine the arrival angle from all $90 \mathrm{~m}$ transmissions at that array depth. As well, the vehicle's statics for receptions from $\mathrm{H} 1\left(z_{s}=90 \mathrm{~m}\right)$ are included.

deep. In this region, the HYCOM approximation appears to track nicely with the DURIP towed array data. Looking at Figure 4-30, there is alignment between the HYCOM approximation and the XCTD. However, the DURIP towed array data does not line up as nicely with these expected arrival angles. There are a few obvious possibilities.

1. The SSP was closer to that predicted by Figure 4-31 HYCOM approximation at the time of the experiment.

2. The real data was within $4^{\circ}$ of the expected arrival angle. It's feasible the divergence was due to the array canting so that it was not perfectly vertical. The canting angle 


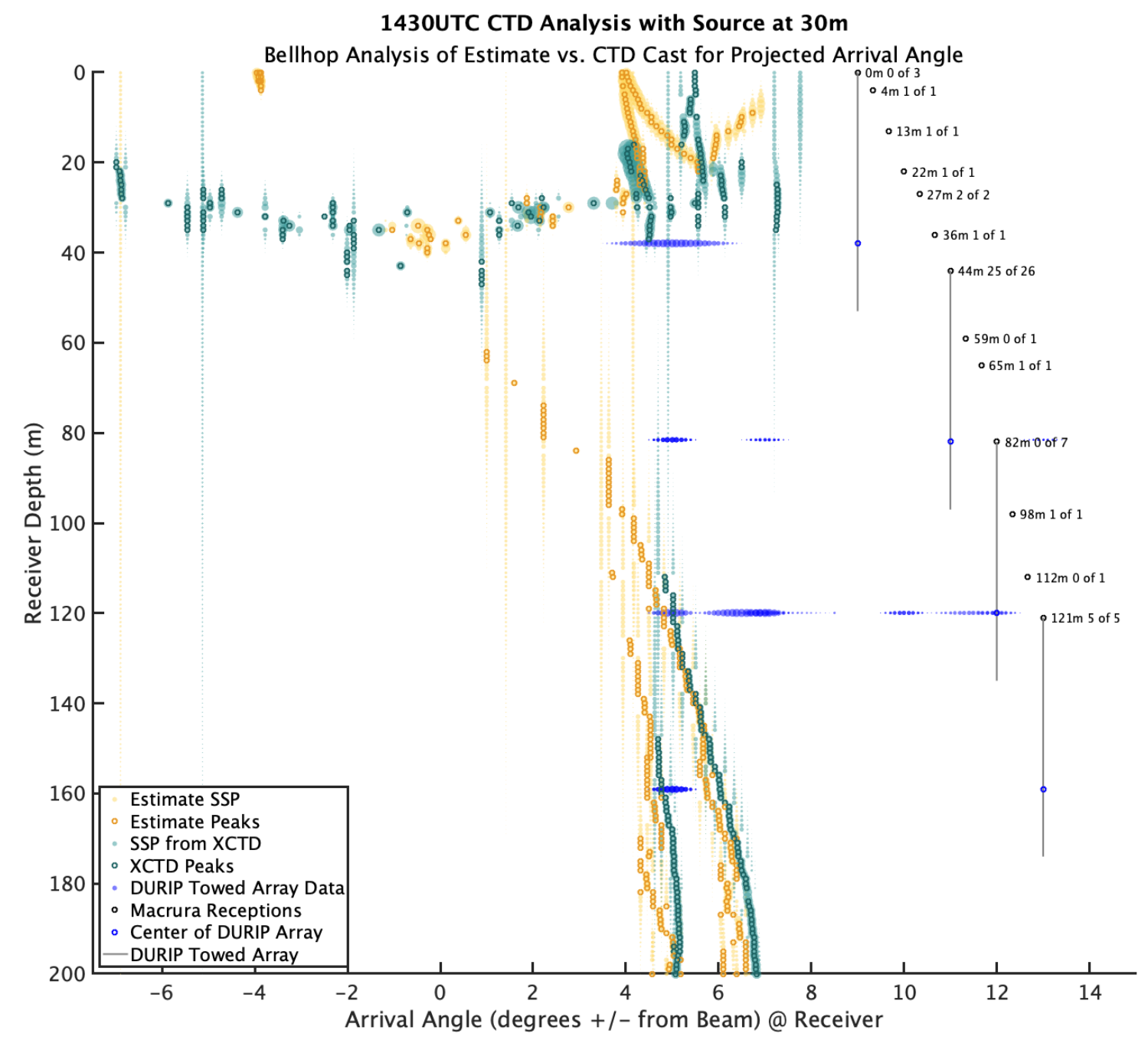

Figure 4-32: Expected arrival angle from the XCTD from 1430 vs. the HYCOM approximation for the SSP for a $30 \mathrm{~m}$ source depth. DURIP towed array data was used to determine the arrival angle from all $30 \mathrm{~m}$ transmissions at that array depth. As well, the vehicle's statics for receptions from $\mathrm{H} 2\left(z_{s}=30 \mathrm{~m}\right)$ are included.

shifts all of the arrival angles.

3. Figures 4-30 and 4-31 make the arrival angle look incredibly narrow, but considering when beam forming the HPBW is $\sim 4.5^{\circ}$, all of these angles are within that error.

Although there are other possibilities, these are most likely. Between $115 \mathrm{~m}$ and $121 \mathrm{~m}$ there is the most variability in correctly demodulated receptions from the vehicle. Looking at the missed transmissions on Figure 4-14 while the vehicle was at those depths, it's clear that the vehicle received those transmissions, but they weren't correctly decoded. This checks with the amount of variability we see on both figures for arrival angles. 


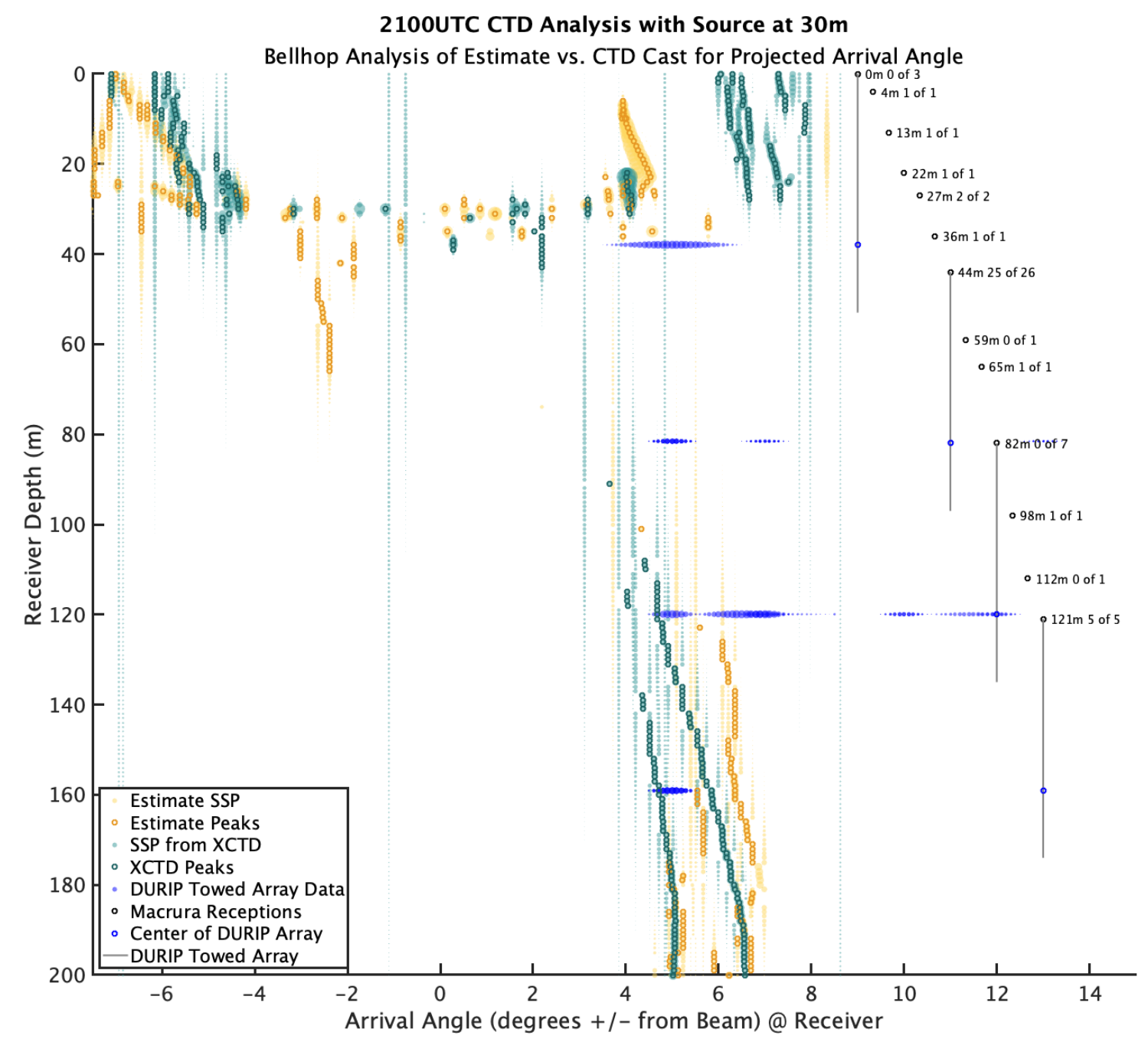

Figure 4-33: Expected arrival angle from the XCTD from 2100 vs. the HYCOM approximation for the SSP for a $30 \mathrm{~m}$ source depth. DURIP towed array data was used to determine the arrival angle from all $30 \mathrm{~m}$ transmissions at that array depth. As well, the vehicle's statics for receptions from $\mathrm{H} 2\left(z_{s}=30 \mathrm{~m}\right)$ are included.

Without a single strong or clear arrival path, its far more likely that the signal undergoes some constructive or destructive interference that prevents good signal demodulation. Multiple arrival paths, just slightly off from one another, could result in signal interference at the micro-modem which complicates demodulating the signal. This can be seen in Figure 4-15 when looking at the number of different arrival trajectories near those depths. Looking at transmission \#1653, Figure 4-23, with the array at $121 \mathrm{~m}$, the transmission from the buoy does not show up sharply. Instead, they're more broad and diffuse (swathy). Based on this analysis, it may have been better to operate the vehicle around 130 to $160 \mathrm{~m}$. These depths correlate with transmission \#1773, 1803, 1818, 1833, and 1923 (Figures 4-24, 4-25, 4-26, 
4-27, and 4-28). Looking at these transmissions on the DURIP towed array, they appear clean, and would likely demodulate without any issues.

\subsubsection{H2 Analysis with Source Depth, $z_{s},=30 \mathrm{~m}$}

Regarding Figures 4-32 and 4-33 for a source at $30 \mathrm{~m}$, the plots don't significantly diverge from one another. Generally they are similar to one another, although the 1430UTC XCTD and HYCOM approximation track more closely together. It's clear that the DURIP array data tracks well with the projections while the array is shallow. For example, transmission \#273, Figure 4-18, is not as sharp or explicitly defined likely due to the variability seen in the anticipated arrival angles around this depth.

While the DURIP towed array was at depth orange $(\sim 81.5 \mathrm{~m})$ the transmissions were incredibly weak (transmission \#873 and 963, Figures 4-20 and 4-21). This is in alignment with the sparse projected arrival angles between 50 and $115 \mathrm{~m}$ on both Figures 4-32 and 4-33. This aligns with the intermittently demodulated transmissions at those depths (only 2 of 11 transmissions in those depths were correctly demodulated). Once below that, near $121 \mathrm{~m}$, the vehicle successfully decoded far more signals. Those were similar to what is seen in transmission \#1458, Figure 4-22. From this analysis, between 50 to $115 \mathrm{~m}$ good communications between the micro-modems and the vehicle are unlikely due to the sparse arrivals at those depths. As a result, at those depths its better to communicate with a 90 $\mathrm{m}$ source than a $30 \mathrm{~m}$ source.

Lastly, while the array was at nearly $159 \mathrm{~m}$ depth, the transmission from the $30 \mathrm{~m}$ source checks pretty clearly with the anticipated arrival from both Figures 4-32 and 4-33. Although the figures show that there is a subtle arrival path difference, looking at transmission \#2463, Figure 4-29, it's unclear that there are two different arrivals. Instead these two arrivals appear to merge into one, and the beam formed data only shows one likely arrival path. 
THIS PAGE INTENTIONALLY LEFT BLANK 


\section{Chapter 5}

\section{Communications Signal Analysis}

After focusing on the Vertical Run portion of the data, I noticed some some interesting patterns. I opened up my aperture to the entire data set for further analysis.

\subsection{One Way Travel Time (OWTT)}

Throughout the experiment a Time Division Multiple Access (TDMA) communications network was employed. This allows the vehicle and buoys to communicate with one another without talking over one another. The vehicle transmits at XX:XX:16, and XX:XX:46. The WHOI micro-modems transmit at XX:XX:01 and XX:XX:31 (only 1 of the 4 buoys will transmit during a buoy transmission window). The 15 seconds between transmissions is the window of time for the signal to propagate through the water. The furthest you could communicate with a 15 second window is $d=r t \Rightarrow d=1500 \mathrm{~m} / \mathrm{s}(15 \mathrm{~s}) \approx 22500 \mathrm{~m}$. This is a perfect scenario where acoustic waves travel in straight lines at the same speed like electromagnetic waves. Operationally a 15 second TDMA window is for communicating inside of $20 \mathrm{~km}$. The buoys don't necessarily transmit every time, but the vehicle does transmit every communications window. Also, for any given transmission from a buoy, the other buoys potentially receive that transmission as well.

Lastly, there is a message posted when the WHOI micro-modem triggers on a possible reception. This message provides the communications cycle receive statistics "CACST" for the message. Most importantly, one of the first fields (field \#2) is if the message was good or bad. This determination is based on the Cyclic-Redundancy Check (CRC) [31]. The CRCs are similar to a check-sums and used to validate the correct decoding of a transmission. 
The message is considered good if there were no CRC errors. However, there are plenty of messages that are potentially viable to improve our under ice navigation accuracy, but include a CRC error that subsequently makes the entire message bad.

Each transmission carries a significant amount of information. Although, for the improved navigational accuracy of this network, only one buoy needs to correctly decode the message from Macrura. It's not necessary that each buoy perfectly decode the message from Macrura. Instead, the most important piece of information is the timestamp of arrival (TOA) for the message at the WHOI micro-modem. Since each buoy has a GPS and clock sync, the message TOA is used in conjunction with the TDMA window to determine a One Way Travel Time (OWTT) for the message. For example, a message arrives at XX:XX:47.321 from Macrura, then the OWTT is calculated as 47.321 - 46.0 = 1.321 seconds. This OWTT is used to calculate a range from that buoy. This range is a circle as seen in Figure 3-8. The more buoys that receive the transmission from Macrura means a more accurate vehicle fix since more circles are plotted overlapping. For a given transmission from Macrura, as long as one buoy decodes the message, the other buoys do not need the message content, but a TOA in order to improve our navigational fix accuracy. It's important that we toss out spurious detections so wrong TOAs are rejected.

\subsubsection{OWTT Potential Transmissions}

Looking at the buoy data during the vertical run, I found multiple transmissions that were decoded by the WHOI micro-modems with CRC errors in the message content. This meant the TOA was not used from that buoy for navigation purposes. Our code looked at the message TOA, the source, and destination (ie. who transmitted and who was the intended recipient). With that information the TOA and TDMA window were used to calculate a OWTT. Instead of focusing on the entire message content as good or bad, I went through the additional fields that are included in the "CACST" messages to find a way to remove superfluous detections. One particularly interesting field, \#29, was the carrier frequency. I noticed it was only $10000 \mathrm{~Hz}$ in some of the cases that were bad. When I compared those OWTTs to what the expected OWTT should have been based on the vehicles position, it was clear that those messages weren't spurious detections from the micro-modems that needed to be thrown out.

I took all of the buoy receptions throughout the entire ICEX experiment in March and 
did a histogram of the OWTTs. When I did this I subtracted the 1 second delay that occurs, so the Buoy Transmission Window is from 0 to 15 seconds, and the OWTT is the actual time on the $\mathrm{x}$-axis. The Macrura TDMA transmission window is from 15 to 30 seconds, and the OWTT is calculated from the time on the x-axis minus the 15 second offset.

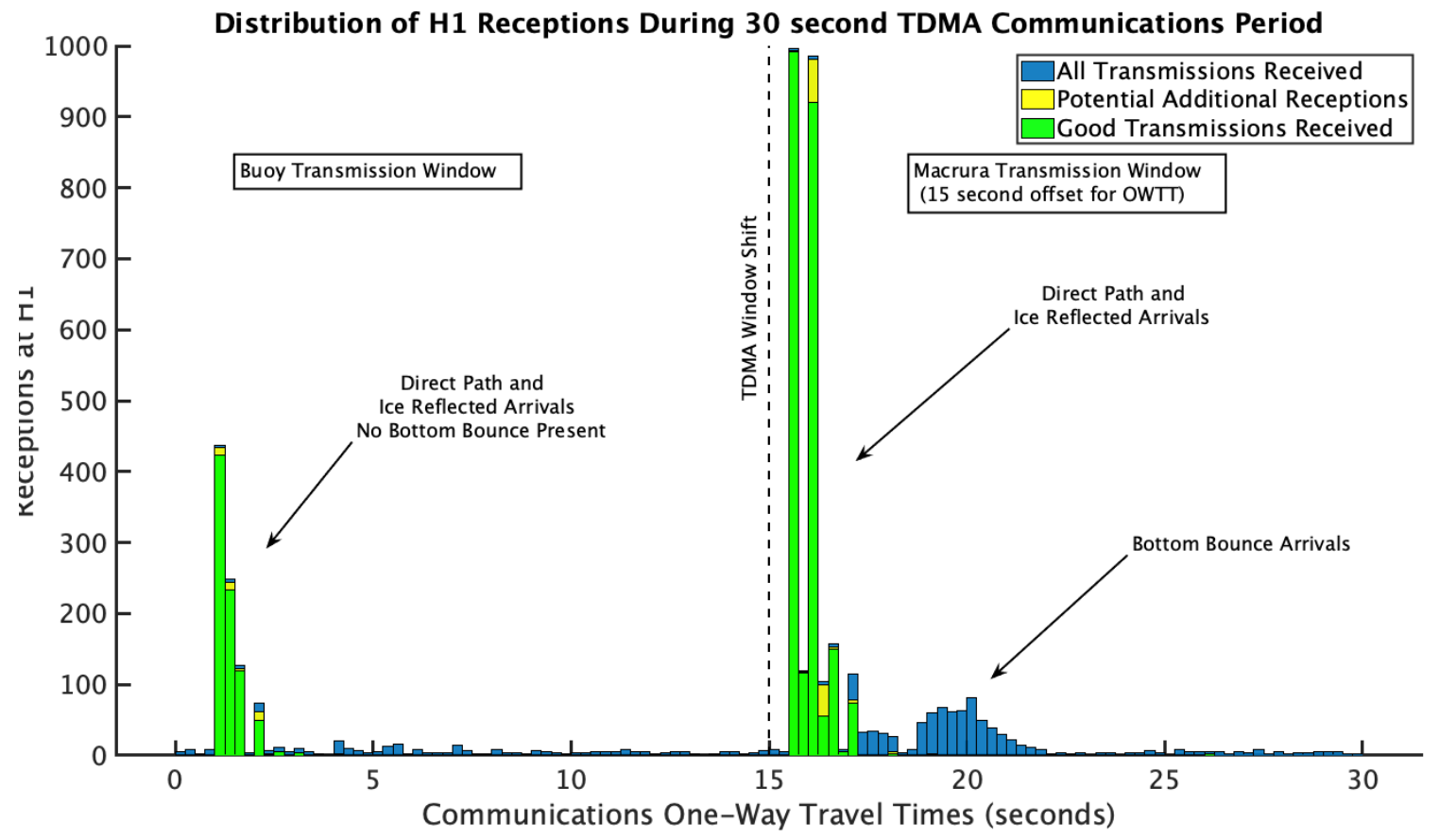

Figure 5-1: Histogram of H1 arrivals throughout ICEX.

Figures 5-1, 5-2, 5-3, and 5-4 highlight the potentially viable additional transmissions in yellow. The focus here is on the vehicle transmission window, between 15 to 30 seconds. There is a reasonable number of additional receptions that if used would aid in the navigational accuracy of the vehicle throughout its operations. Although these messages aren't perfectly decoded, checking for the correct carrier frequency of $10000 \mathrm{~Hz}$ in the "CACST" message is good enough to isolate these potentially viable OWTTs which translates to an improvement in the navigational accuracy.

\subsection{Arrival Path}

Based on ray traces shown in Figures 4-15, and 4-16, the expected arrival paths are direct path or include a possible surface reflection. None of the bottom bounce (BB) trajectories were even included in the ray traces because transmission angles in excess of 70 degrees were necessary for a BB arrival path which seemed unlikely. Based on the OWTT for the 


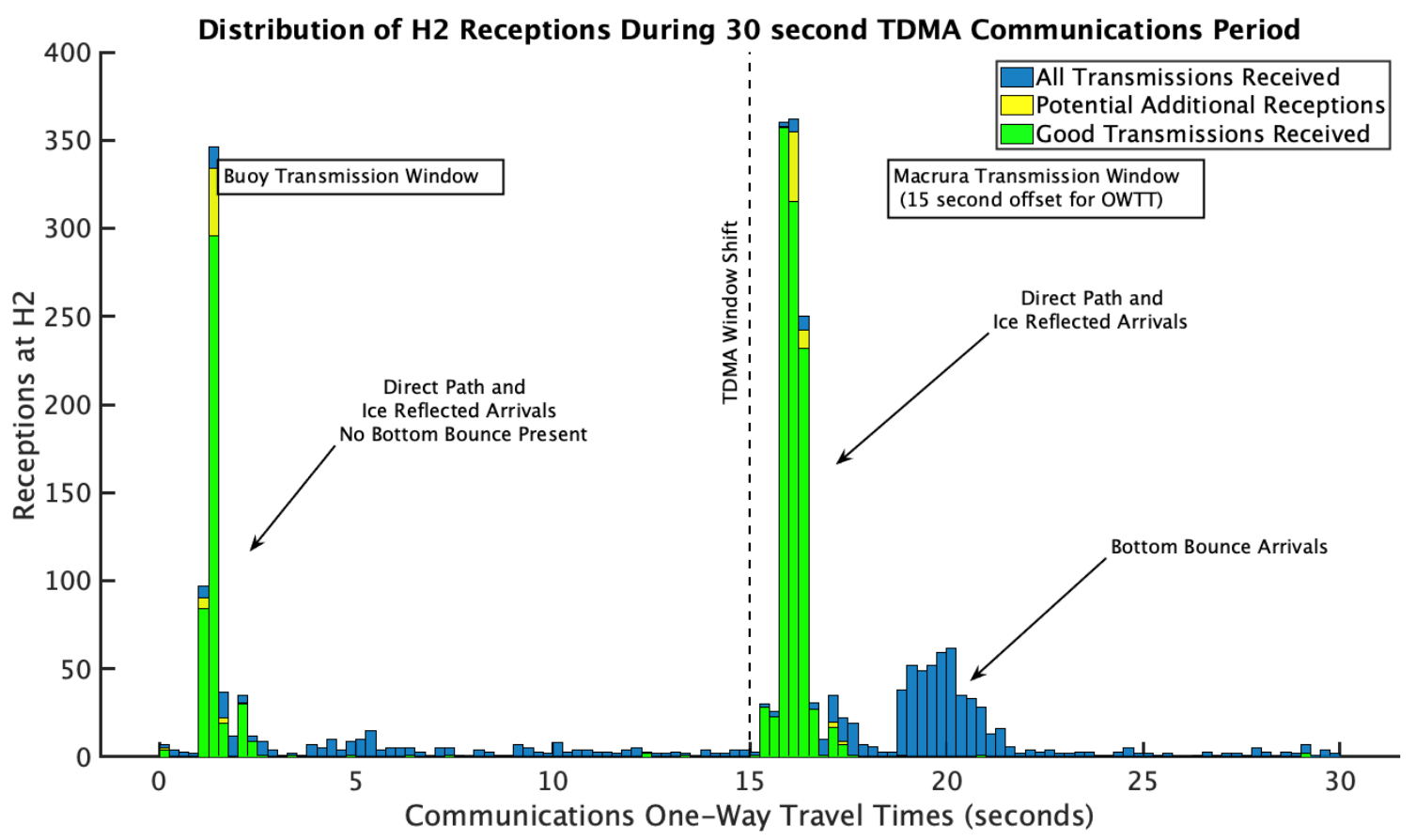

Figure 5-2: Histogram of H2 arrivals throughout ICEX.

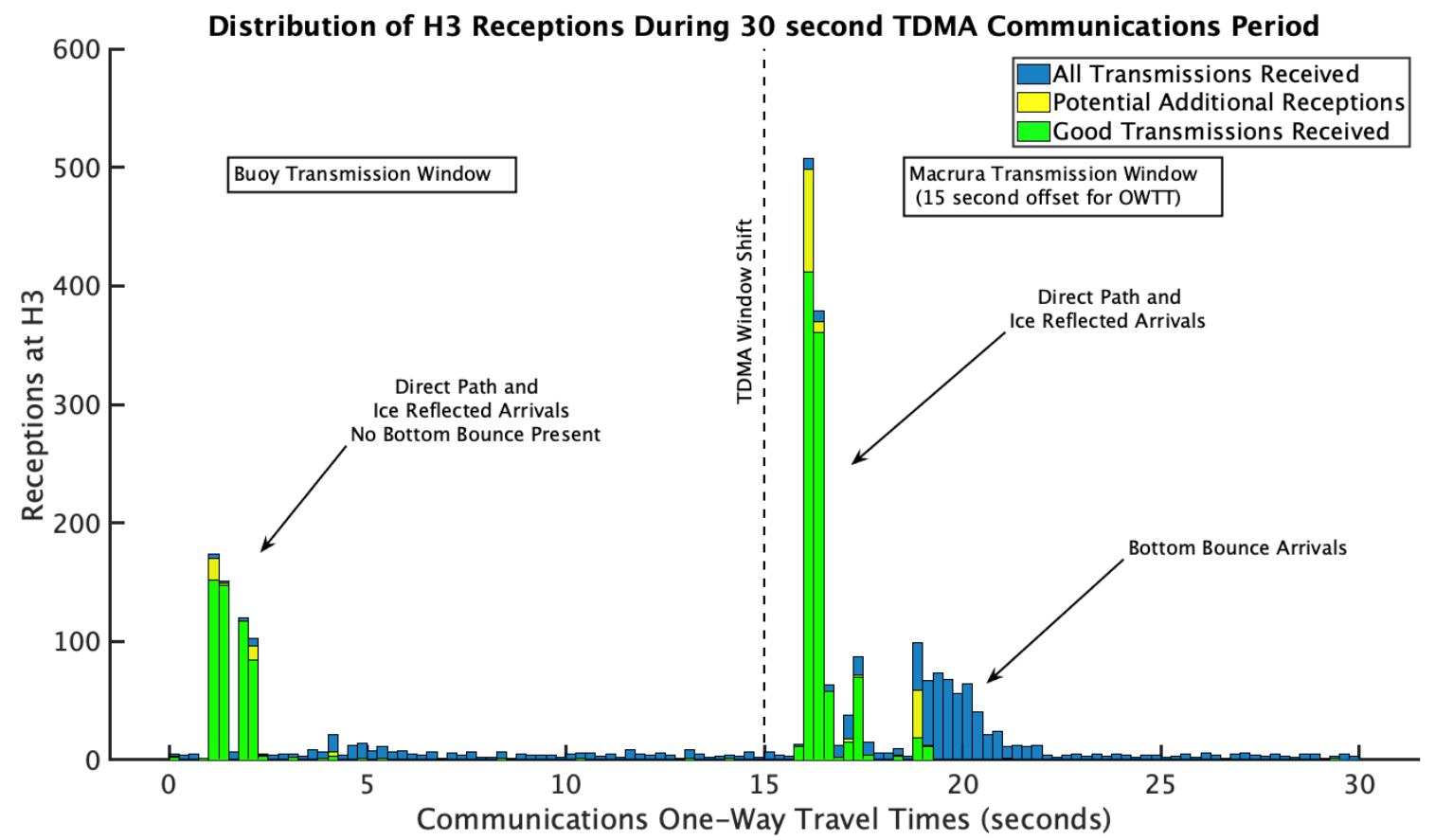

Figure 5-3: Histogram of H3 arrivals throughout ICEX.

signals, almost all arrivals were direct path or include a surface reflection, which is in line with the prediction from the ray traces that this angle was too steep, and too much signal attenuation would occur. 


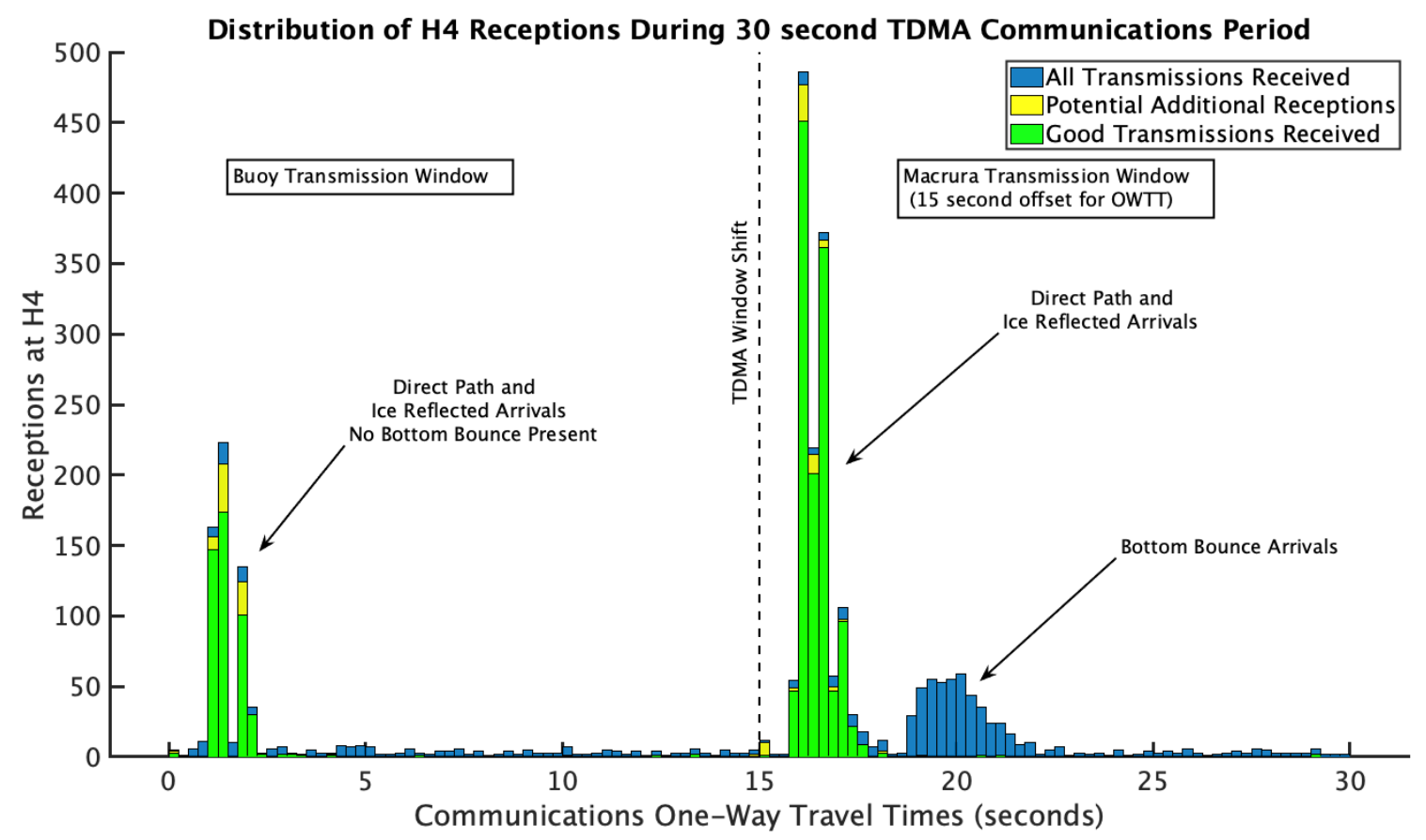

Figure 5-4: Histogram of H4 arrivals throughout ICEX.

The BB trajectory requires a OWTT of at least 3.5 seconds. This is based on the total water depth (TWD) in the area of operations for ICEX. Figure 5-5 shows the bathymetry in the area. The shallowest depth expected was around $2620 \mathrm{~m}$ deep. The time it takes a signal to go to the bottom and return is $t=\frac{2 * 2620 \mathrm{~m}}{1500 \mathrm{~m} / \mathrm{s}} \approx 3.5 \mathrm{sec}$. Any OWTT less than 3.5 seconds must be a direct path or surface reflected path. OWTT in excess of $3.5 \mathrm{sec}$ is potentially a BB arrival.

Note: Throughout the operations period there was no time when the vehicle operated at a range which would make sense for a direct path or surface reflected OWTT in excess of 3.5 seconds.

\subsubsection{Buoy Transmission Window Analysis}

There are two TDMA windows to look at separately. First, the buoy transmission window includes no arrivals with a bottom bounce trajectory. This is clear because the number of receptions in excess of 3.5 seconds is negligible. This is true for all of the transmissions from the WHOI micro-modem buoys. These buoys have a transmission pattern similar to a dipole or a donut shape. The majority of the buoy's energy is transmitted with an outward trajectory, and less energy is transmitted up or down in the water column. As a result, the 


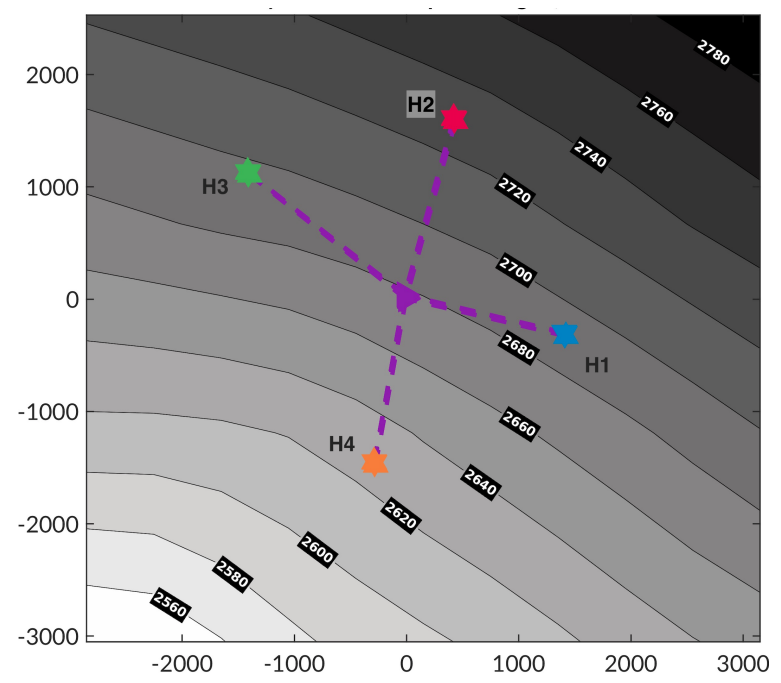

Figure 5-5: Bathymetry at Ice Camp Seadragon [27].

$\mathrm{BB}$ arrival path is less likely, and this is captured in the histograms.

\subsubsection{Macrura Transmission Window Analysis}

The second TDMA window looks at transmissions from Macrura. In this window there is a substantially different pattern than from the buoy transmissions. A second hump centered around 20 seconds are the arrivals that include some BB trajectory. This makes more sense considering the transmission pattern and placement of the $10 \mathrm{kHz}$ transducer on the vehicle. The transducer has a hemispherical transmission pattern and is located on the bottom of the vehicle. With the transducer upside down from its normal configuration due to operating under the ice, more energy is transmitted with steep downward trajectories, and more of those signals are present with OWTTs at the buoys in excess of 3.5 seconds. However, like we assumed, most of these transmissions were too badly attenuated to be effectively utilized.

All of the transmissions received at the buoys in excess of 3.5 seconds are from $\mathrm{BB}$; $\mathrm{BB}$ to surface reflections; or surface reflections to $\mathrm{BB}$ (unlikely due to the $10 \mathrm{kHz}$ transducer mounting on the bottom of Macrura for ICEX). As a result of the additional attenuation and scattering from bottom interactions it's no surprise that almost all of these transmissions were bad and did not decoded correctly. The long trajectory coupled with the interaction with the bottom results in significant signal degradation. Buoy H3 is an outlier to this trend, and Figure 5-3 is quite different than the other histograms. A large portion of the BB hump includes good receptions. As well, there are a large number of receptions that are 
potentially viable for OWTT calculations.

\subsection{Wave-Front Range Analysis from OWTT}

Further analysis on the transmissions that arrived at buoy H3 with a OWTT in excess of 3.5 seconds resulted in some interesting findings. The analysis was done using a version of the code developed by Dr. Eeshan Bhatt for his thesis, A Virtual Ocean Framework for Environmentally Adaptive, Embedded Acoustic Navigation on Autonomous Underwater [27].

Here the total water depth (TWD), number of rays and launch angles $\left(-40^{\circ}\right.$ to $+80^{\circ}$ at $1^{\circ}$ intervals), a source depth (Macrura's depth), and SSP are defined. The SSP used was from the SSP estimate employed on board Macrura that was modeled after the 1430UTC XCTD. Unlike with BELLHOP, the OWTT is specified. This OWTT is used to plot the leading edge of the acoustic wave-front at that moment in time. It's effectively following the ray paths until a specified moment in time, and then plotting the resulting curve, which is equivalent to the Wave-Front.

Figure 5-6a is from the Vertical Run. The direct path OWTT was based on a direct path or surface reflected arrival at H3 while Macrura was at $82 \mathrm{~m}$ deep. While the vehicle was at $121 \mathrm{~m}$, all of the receptions at $\mathrm{H} 3$ were from $\mathrm{BB}$ arrival paths. The dashed line is the depth of the $\mathrm{H} 3$ deep receiver $(90 \mathrm{~m})$ that was employed at the time, and the red $\mathrm{X}$ is the position of buoy H3. Figure 5-6b zooms in on the wave-front near buoy H3. From this perspective it's impressive how close the wave-front is to the location of buoy H3.

It's important to note, looking at Figure 5-6a, if the bottom bounce arrival path is neglected, the range for a OWTT of 3.7633 seconds is roughly $5500 \mathrm{~m}$. This is where the leading edge of the orange wave-front is intersecting with the $90 \mathrm{~m}$ dashed line. So when topside calculates a range ring for this OWTT, it doesn't match at all with the OWTT range rings from the other nodes, and the fix is effectively thrown out. Instead, the goal would be to find a way to take advantage of the potential BB arrival path for the fix. This would result in a far more accurate fix and a better overall solution.

\subsubsection{Total Water Depth}

Although the SSP is an approximation, the biggest unknown here is the Total Water Depth (TWD). This analysis assumes a hard flat bottom. Minor adjustments in the TWD result 

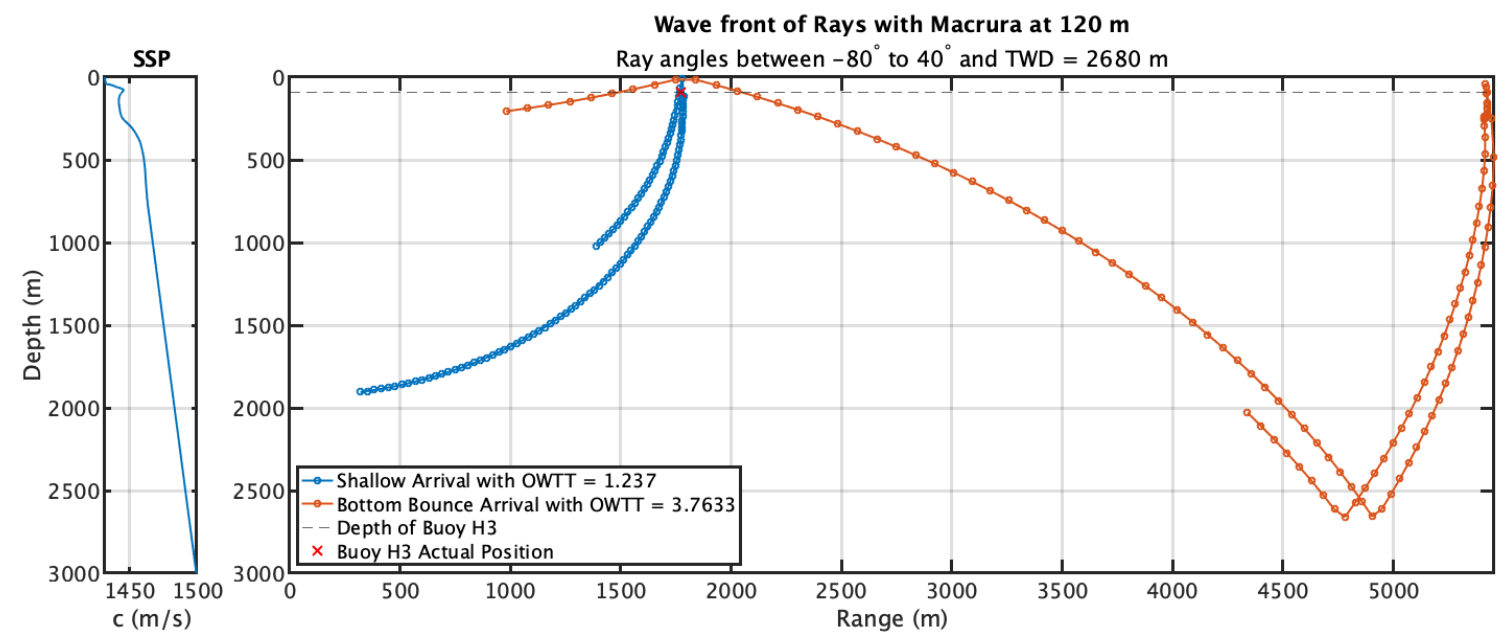

(a) Wave-Front of Macrura transmission based on the OWTT. Blue curve represents the Wave-Front from a direct path or surface reflected arrival at H3. Orange curve represents the Wave-Front from a $\mathrm{BB}$ arrival at $\mathrm{H} 3$.

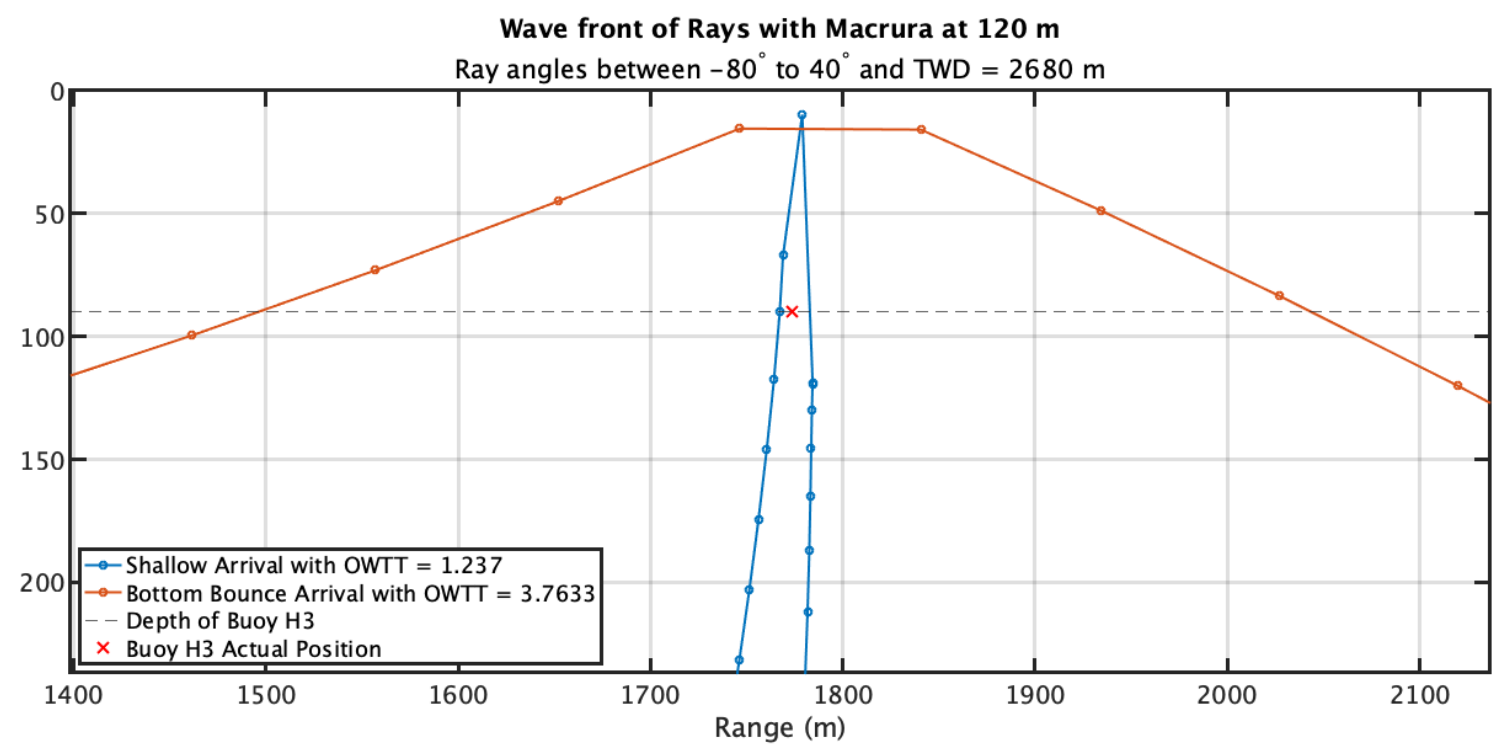

(b) Zoomed in on the Wave-Front from Macrura's transmission. The red X represent buoy H3, and it's position relative to the wave motion.

Figure 5-6: Wave-Front of Macrura transmission based on OWTT during the Vertical Run. The direct or surface reflected OWTT was based off a reception while the vehicle was operating at $82 \mathrm{~m}$ since no direct or surface reflected OWTT occurred while the vehicle was at $121 \mathrm{~m}$ depth.

in substantially different arrival ranges. Adjusting the $\pm 50 \mathrm{~m}$ results in covering the spread of viable solutions. Figure 5-7 shows the difference in these TWD wave-fronts.

A difference of $50 \mathrm{~m}$ is less than a $2 \%$ error in the TWD for the area. However, based on Figure 5-7, and the probability that the correctly decoded arrival likely incurred only a single interaction with the bottom I selected a new TWD of $2730 \mathrm{~m}$. 


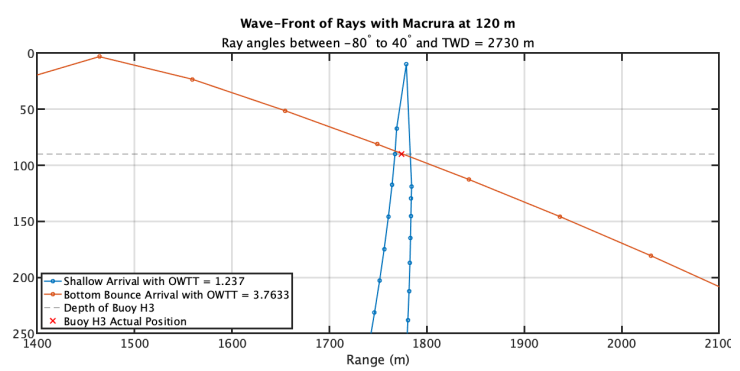

(a) Adjusted TWD to $2730 \mathrm{~m}$. This is equivalent to a $\mathrm{BB}$ reflection arriving at buoy $\mathrm{H} 3$. Most probable since only one interaction vice two.

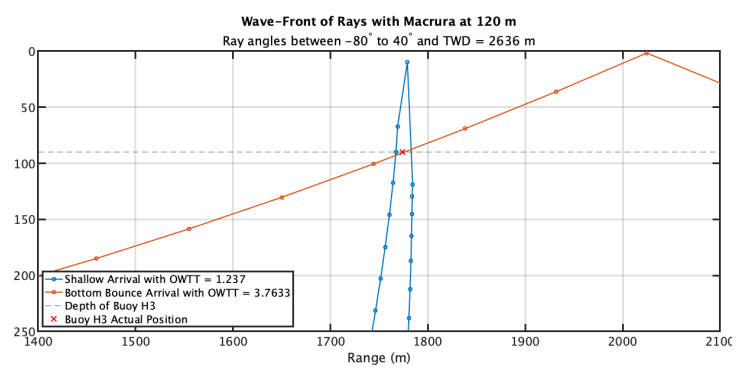

(b) Adjusted TWD to $2636 \mathrm{~m}$. This is equivalent to a bottom bounce to surface reflection then arriving at buoy H3. Less probable.

Figure 5-7: Spread of TWD that makes sense for OWTT arrival at buoy H3. Note the TWD adjustments have no bearing on the direct path or surface reflected arrival path to H3 (blue curve).

\subsubsection{OWTT BB vs Direct Path or Surface Reflected Ranges}

Subsequently, I found other transmissions throughout the exercise where a BB arrival was present with a direct path or surface reflected path arrival close by in time for a comparison between the range from both solutions. The range between the vehicle and the buoy was always based on the range between Macrura's calculated position and the buoy position. This does potentially incur some error as the vehicle is operating submerged in the Arctic without GPS, but because of the new navigation filter onboard the vehicle, the range tends to be extremely accurate.

The following are wave-fronts for $\mathrm{BB}$ arrivals at $\mathrm{H} 3$, that were also received by $\mathrm{H} 3$ as a direct path or surface reflected transmission. These were not common. Since these particular transmissions include both the short OWTT and the BB, they were good comparisons to one another. For all of these analyses since the transmission was received at H3 a TWD of $2730 \mathrm{~m}$ was used. Figure 5-8 compares two transmissions that both arrive with good "CACST" messages, and Figure 5-9 compares two transmissions that the BB arrival was bad according to the "CACST" message, but was viable according to the $10000 \mathrm{~Hz}$ carrier frequency criteria.

In each of these instances, the range is where the direct path or surface reflected path is crossing the $90 \mathrm{~m}$ receiver depth or the distance where the $\mathrm{BB}$ is crossing the $90 \mathrm{~m}$ receiver depth. The difference between the BB range and the Macrura solution range never exceeded the difference between the direct path or surface reflected range and Macrura's solution range by more than an absolute distance of $10 \mathrm{~m}$. That's shockingly close for a BB arrival path, 


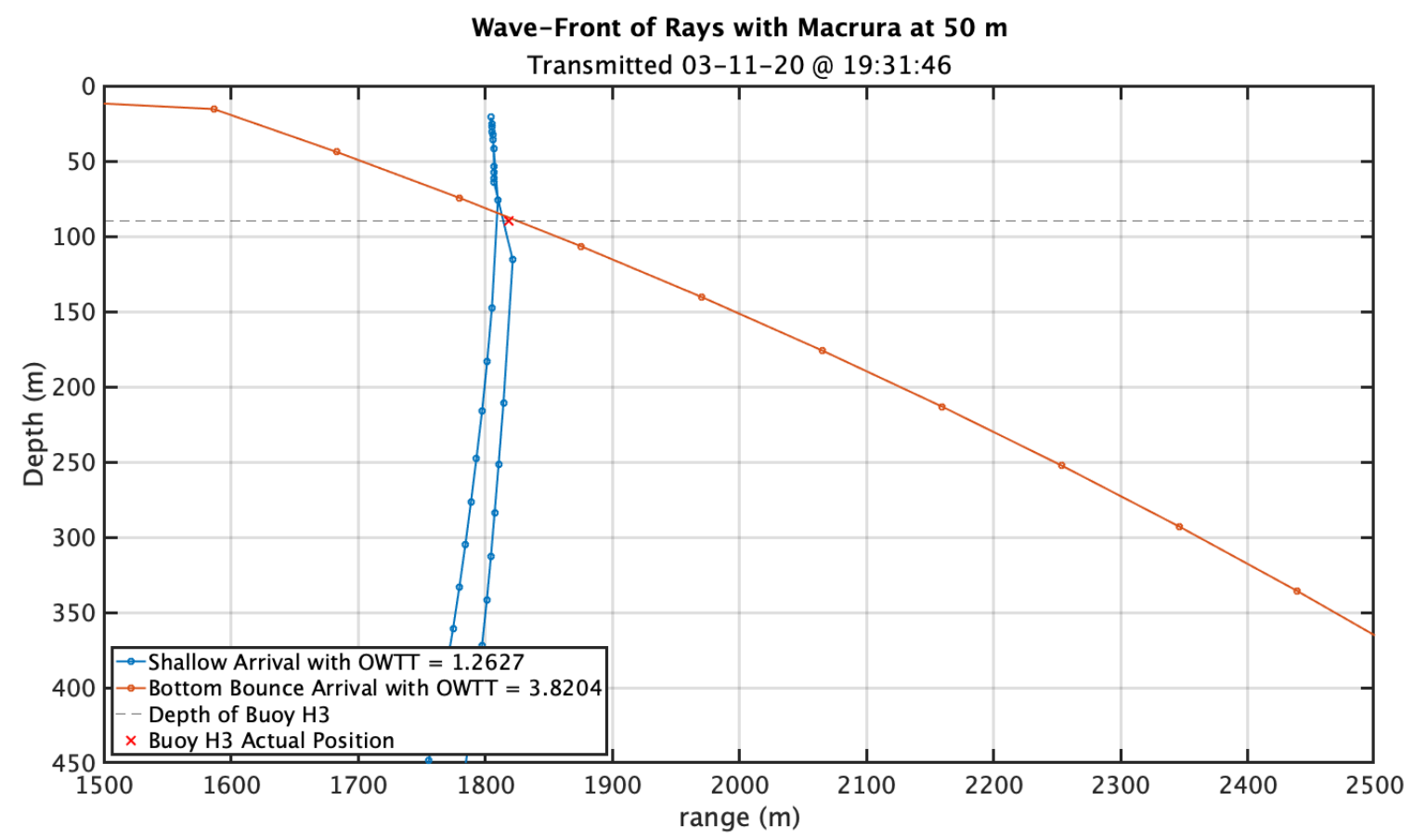

(a) The both arrivals were both good according to the "CACST" message. The difference between the direct path or surface reflected range and the $\mathrm{BB}$ range to buoy $\mathrm{H} 3$ is minimal.

Wave-Front of Rays with Macrura at $\mathbf{4 0} \mathbf{~ m}$

(b) The both arrivals were both good according to the "CACST" message. The difference between the direct path or surface reflected range and the $\mathrm{BB}$ range to buoy $\mathrm{H} 3$ is minimal.

Figure 5-8: OWTT comparison for the same transmission received twice at buoy H3. 


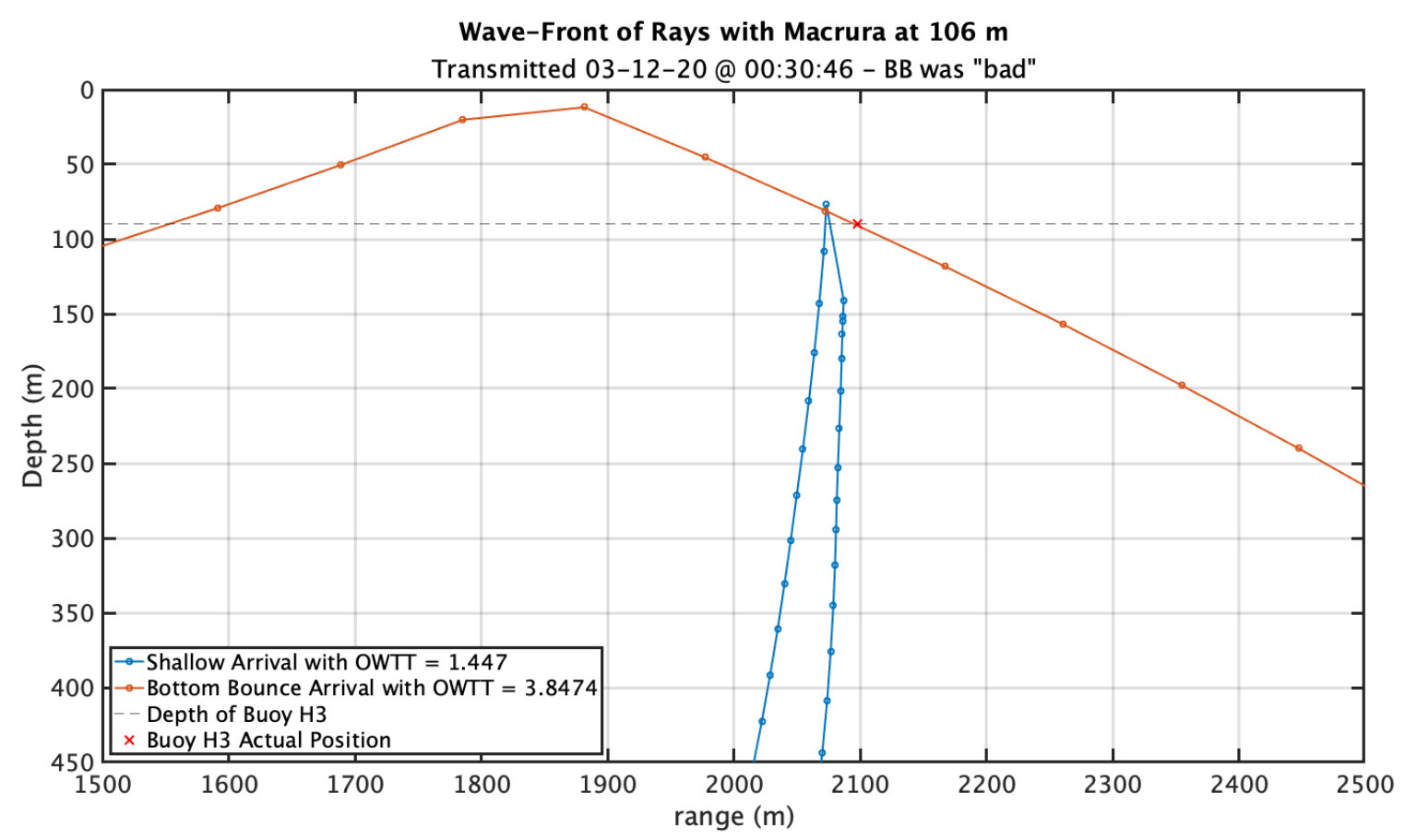

(a) The BB arrival was not considered a "good" reception according to the "CACST" message.

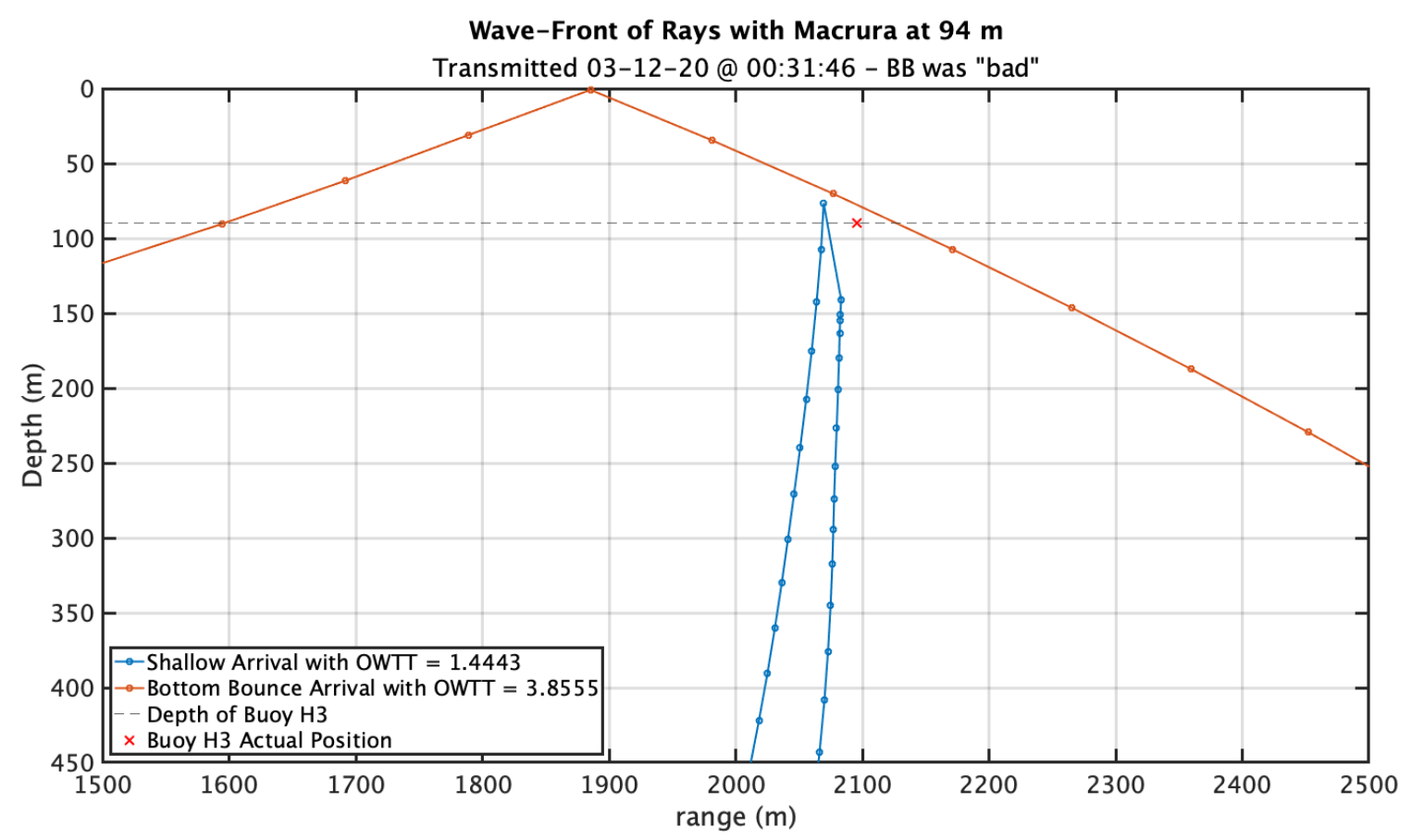

(b) The BB arrival was not considered a "good" reception according to the "CACST" message.

Figure 5-9: OWTT comparison for the same transmission received twice at buoy H3, but the BB arrival was "bad" according to "CACST" but was viable according to the carrier frequency of $10000 \mathrm{~Hz}$. These were both yellow in Figure 5-3. 
and it shows that the BB arrivals are not only viable for acoustic communications, but are a potentially reliable source for ranging. This means more transmissions could have been included in improving the topside navigation solution.

I wanted to find an additional BB arrival from one of the other buoys other than H3. There were three key problems that made this difficult, and were part of the reasons BB arrivals at buoy $\mathrm{H} 3$ were possible:

1. Range: H3 was the furthest buoy from most of the AUV's operations. This means that the ray launch angles from the vehicle were not as steep, resulting in less scattering from the bottom interactions.

2. Bottom Type: Along the path between the vehicle and buoy H3, the bottom type was hard enough that the transmission was able to reflect off the bottom vice be entirely scattered or absorbed.

3. Bottom Contours: Along the trajectory between the vehicle and bouy H3, the depth appeared fairly flat based on the bathymetry.

Although those facts were working against the other nodes, I found another BB transmission that arrived at buoy H2. This occurred while Macrura was completing the un-tethered operational run and was operating up to the NE, far enough from $\mathrm{H} 2$, that $\mathrm{BB}$ was possible, and near the same depth contour to buoy H2. Figure 5-10 shows this transmission. The "CACST" message for the BB arrival path showed that the message was bad, but according to the alternate frequency criteria, the transmission was potentially viable.

\subsubsection{BB Arrivals from H1 to H3 During Simulated Run}

After further analysis there was one period of data that I needed to re-analyze. During the period we did additional node testing while conducting the minor repairs to the vehicle

discussed in Section 3.3.3. This testing shifted the node numbers for the buoy that was simulated as Macrura to 004 vice the original node number assigned, and I needed to reevaluate these transmission based on this. This test took place on 03-10-2020 between 1700UTC and 2000UTC. There was a single shallow arrival that occurred throughout the duration of the test that arrived at buoy H3 at 17:13:18.2028. OWTT $=2.2028$ seconds. Although this is two hours before the BB arrivals occurred, there was minimal ice motion during the test. 


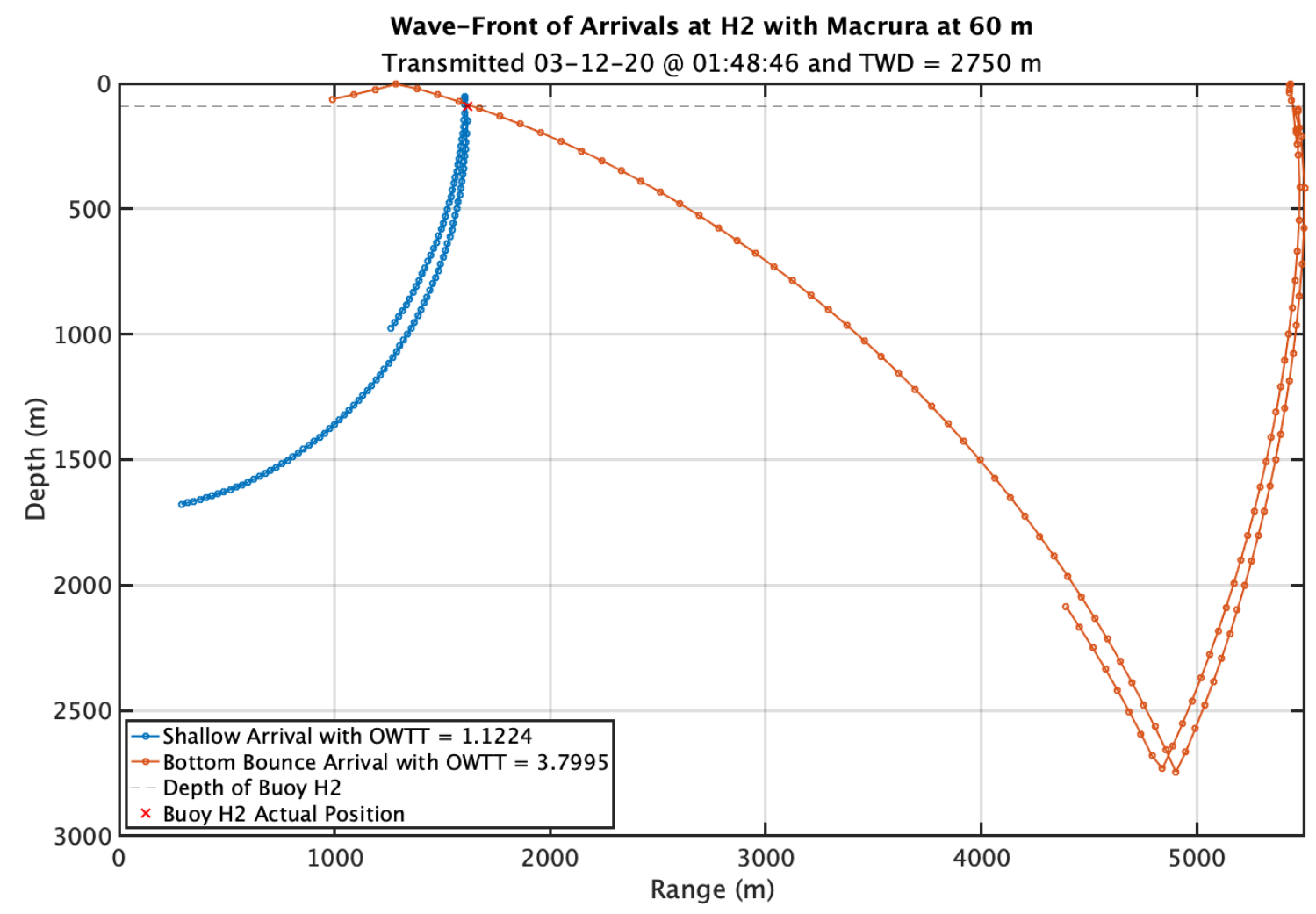

Figure 5-10: The BB transmission at $\mathrm{H} 2$ was not considered a "good" reception according to the "CACST" message. However, based on the $10000 \mathrm{~Hz}$ carrier frequency analysis, the message met the criteria for an acceptable message. This is one of the yellow potentially viable arrivals from Figure 5-2.

While doing this simulated vehicle testing, between 19:17 and 19:25, there were a total of eleven transmissions that were received by buoy H3 originating from buoy H1 $\left(z_{s}=90\right.$ m) while H1 was simulating it was the AUV. The OWTT varied between 4.1896 and 4.1899 seconds over the course of the 11 transmissions. The spread between these two transmission times is indistinguishable although both are plotted in Figure 5-11.

Figure 5-11 highlights why only a single shallow propagation path arrived at buoy H3 throughout the entire simulated exercise between 1700 and 2000UTC. Based on the wavefront for the OWTT of the one and only shallow path arrival, it's clear that no part of the wave-front is actually projected to arrive at either the shallow or deep buoy. Since we know the SSP is changing temporally and spatially, its not unreasonable that one transmission arrived at buoy H3 out of over 300 transmissions that occurred between 1700 and 2000UTC. This also checks with Table 4.2, although it's referring to transmissions that occurred on 03-11-2020. There were 51 total transmissions from H1 and only 4 arrived at H3 throughout 
the Vertical Run.

It's likely that H1 was canting at the right angle, in-line with buoy H3 during this short window between 1917 and 1925UTC. This angle likely enabled sufficient energy to be transmitted at a downward trajectory so that scattering from the bottom didn't prevent the transmissions from returning to buoy H3. In conclusion, the BB arrivals should be considered to improve the total navigational accuracy of the vehicle throughout future experiments. 

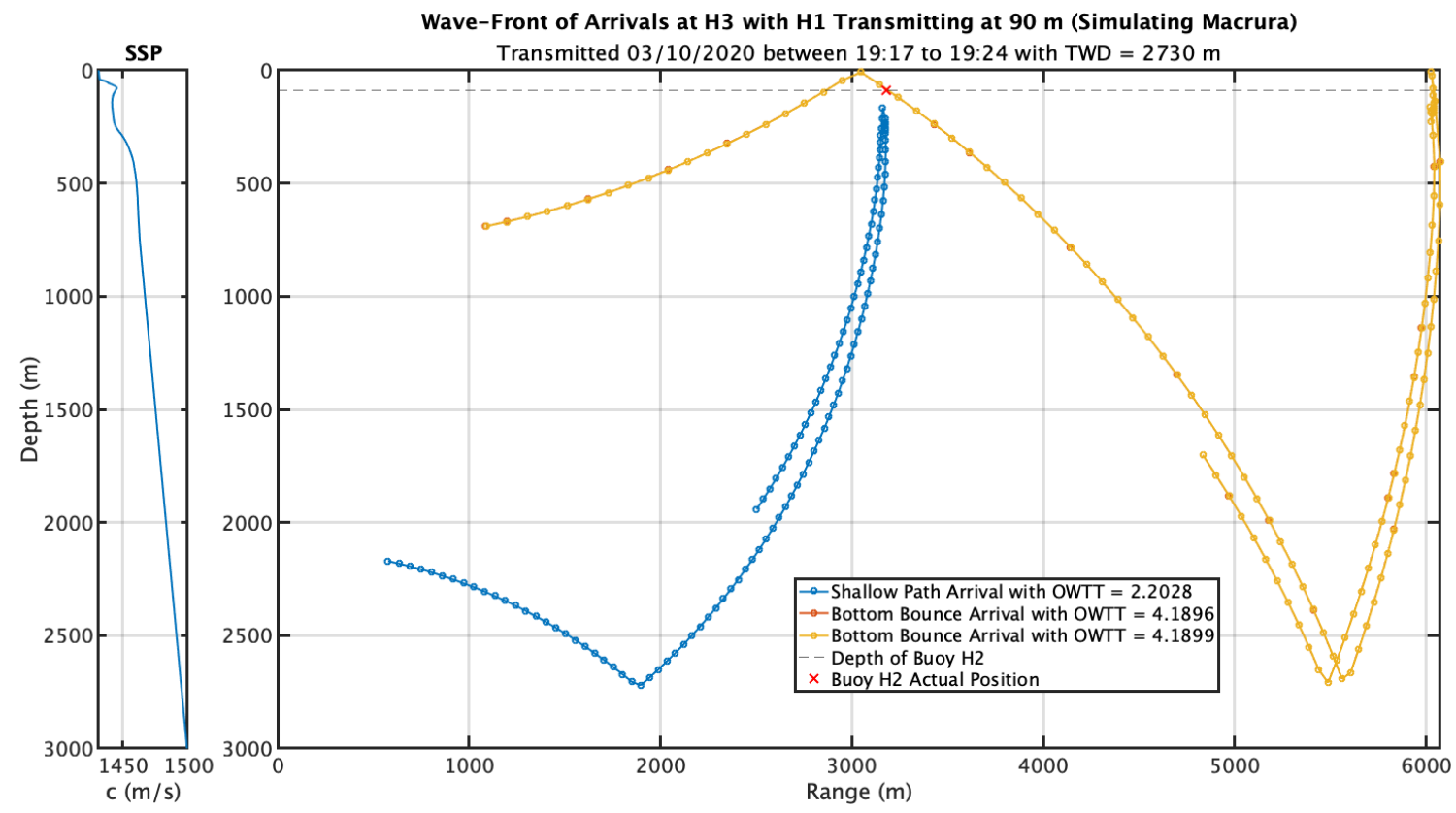

(a) Transmission from H1 to H3. The shallow arrival path is anomalous considering the wave-front doesn't project an arrival at any receiver depth for buoy H3.

Wave-Front of Arrivals at $\mathbf{H 3}$ with $\mathbf{H 1}$ Transmitting at $\mathbf{9 0} \mathbf{~ m}$ (Simulating Macrura)

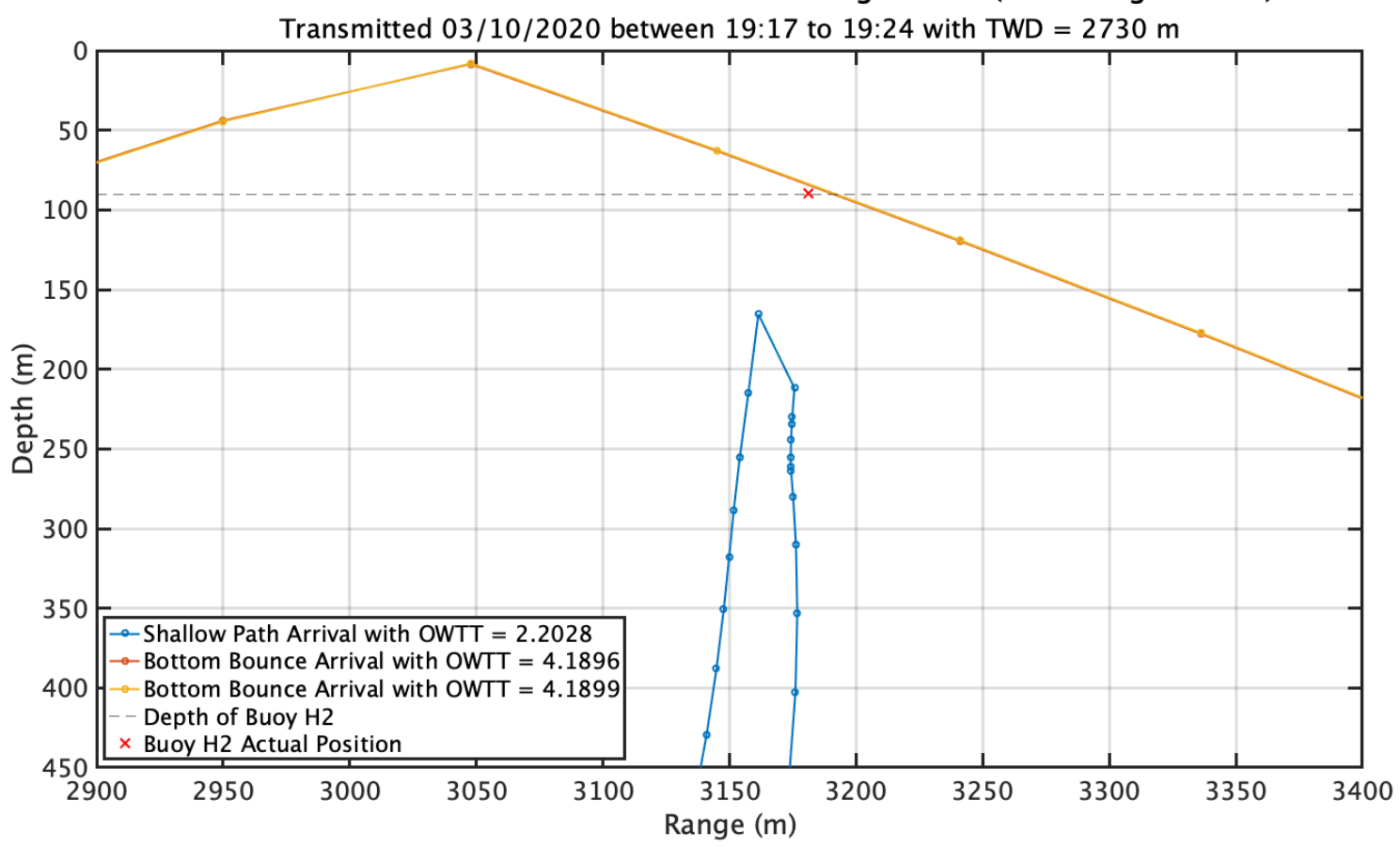

(b) Zoomed in on transmission from $\mathrm{H} 1$ to H3. Shows that the BB arrival path was a reasonable range prediction, and the shallow propagation path is not predicted to arrive at $\mathrm{H} 3$.

Figure 5-11: OWTT comparison for transmissions received at $\mathrm{H} 3$ originating from $\mathrm{H} 1$ while H1 was a simulated vehicle on 03-10-2020. The shallow arrival was from 17:13:16, and the BB arrivals were from 19:17:46 to 19:24:46. Eleven BB arrivals occurred in that window that were all good according to "CACST." 
THIS PAGE INTENTIONALLY LEFT BLANK 


\section{Chapter 6}

\section{Conclusions and Future Work}

\subsection{Review and Conclusion}

ICEX 2020 was an incredible scientific journey. It brought together a team of military personnel, engineers and scientists in a novel way to explore the dynamic environment in the Arctic Ocean. During the experiment, we not only were able to prove that near GPS grade navigation was possible in the Arctic, we showed it under the most trying conditions. We walked out onto the ice, marked a spot via GPS, and subsequently dug a hole through the ice to find the vehicle exactly where we expected it.

The exercise provided the unique framework to compare arrivals from single nodes in a communications network as the buoys and Macrura transmitted back and forth throughout different areas in the Beaufort Lens. Along with this communications network the vertical array created a rare opportunity to generate a third perspective of the communications networks as the vertical array moved through the Beaufort Lens. Although the carrier frequency of the communications network was $10 \mathrm{kHz}$, above the Nyquist frequency of 6 $\mathrm{kHz}$ for the towed array, the subharmonic at $5 \mathrm{kHz}$ was accurately sampled on the array. Next beamforming was done at frequencies above the physical design cutoff frequency for the array based on element spacing. This took advantage of the full frequency spectrum to limit the impact of the grating lobes on isolating the different arrival angles of the acoustic communications transmissions.

Lastly, we took those different paths and compared them to the modeling available from BELLHOP and found the ray trace estimates provided sufficient fidelity to understand what to anticipate in the water column without needing the accuracy of an instantaneous CTD 
to model the SSP. So long as the general shape and speeds of the SSP were close to what was happening in the water column, BELLHOP provided a good approximation for what arrival paths to expect, and which were dominant. These arrival paths were compared with the towed array data and verified to match the modeling.

The final piece was an evaluation of the communications used to range the vehicle throughout the exercise. There were additional transmissions that could be utilized to improve the navigational fix accuracy throughout the exercise. Additionally, bottom bounce arrivals that were not anticipated based on water depth and high frequency communications attenuation were found throughout the exercise. As well, those bottom bounce arrivals were accurate for ranging and could be employed for future missions.

In closing here are the consolidated conclusions as a result of the work contained herein:

1. Under ice navigation in the Arctic was executable with near GPS quality. This enabled walking out to a point on the ice, digging a hole, and recovering the vehicle without the use of divers. This could significantly change torpedo recoveries in the future.

2. AUV recovery does not require a full 3' by $15^{\prime}$ hole for extraction from the ice. With good weather conditions, an exposed vehicle recovery procedure can be executed that allows the vehicle to take a reasonable up angle.

3. The XCTD does not provide additional fidelity over the HYCOM model for BELLHOP analyses.

- NOTE: The HYCOM models were built from the XCTD data. When comparing to what the projected HYCOM SSP for the Arctic was expected to be, there were significant differences.

- It's important to have a recent model of the actual environment to build reasonable ray paths for improved navigation.

4. The Beaufort Lens significantly impacts acoustic communications. Without a transducer above and below the lens, communications with the vehicle are severely hampered based on the vehicle operating depth and range.

5. Although bottom bounce isn't typically considered for deep water high frequency acoustic communications, its clear that this arrival path is real and exploitable to include improved navigation accuracy. 


\subsection{Future Work Recommendations}

Thinking back to ICEX 2018 when egregious amounts of time and manpower were spent looking for exercise torpedoes that were sitting just beneath the Arctic ice, the impacts of this work on future operations are tangible. This communications network employed for navigation is effective in the Arctic Ocean, including in the presence of the Beaufort Lens. There are some minor adjustments that would improve the next AUV operation in the Arctic. The following recommendations are based on the analysis from the ICEX 2020 data:

- Include BB arrivals for range calculations to improve solution fix accuracy.

- Use the fathometer mode from the WHOI micro-modems to calculate actual bottom depth near the modem. The TWD is a large assumption for bottom bounce ranging solutions.

- Use both the shallow and deep hydrophones from the WHOI micro-modems. Each depth, shallow and deep, should be reported independently.

- During ICEX there were 8 receivers in the water with only 4 available at any point during operations. This hampered the total receptions throughout the exercise.

- Consider doing basic beam forming with the two hydrophones that are at the same depth on the WHOI micro-modems. This could enable a basic understanding of the likely arrival path.

- For example, transmissions in excess of $50^{\circ}$ are considered $\mathrm{BB}$, and those within $50^{\circ}$ of the beam $\left(0^{\circ} \mathrm{D} / \mathrm{E}\right)$ are considered direct path or surface reflected.

- This could help distinguish which range to select based on a OWTT.

- Different criteria should be used by topside to ensure all the arrivals that could potentially be used to improve the navigational fix are employed.

- Consider using "CACST" message field \#29, carrier frequency of $10000 \mathrm{~Hz}$, to remove spurious detections. 
- This will improve the accuracy of the topside fixes and subsequently the vehicles internal navigation solution through the feedback from the acoustic communications. 


\section{Appendix A}

\section{Vehicle Recovery Procedure}

The largest concern while recovering the vehicle was to limit the angle the vehicle came out of the water. The vehicle was not designed for removal at an angle. We took advantage of the positive buoyancy of the vehicle, and settled on the FWD end first since the vehicle was light FWD (ie. tended to settle in the water with a positive up-angle). The ASL team executed the recovery procedure as written without any problems. Diagrams used for briefing the team on decisions are shown in Figure A-1.

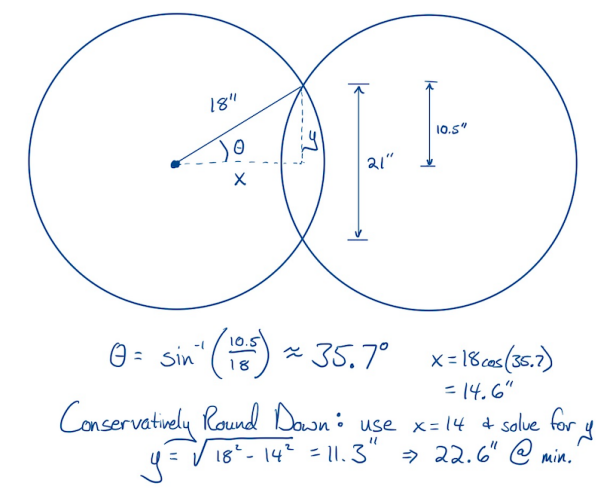

(a) Hole overlap plan. 28" between the holes provided space at the narrowest gap for Macrura to fit through.

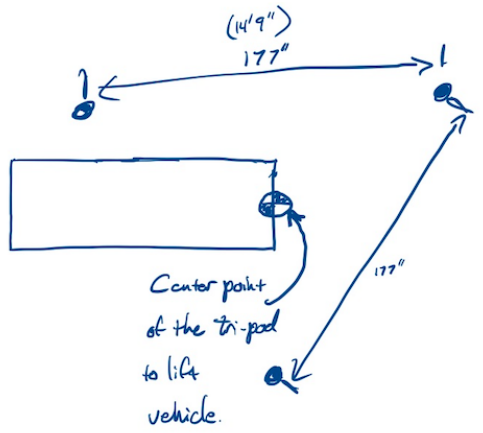

(b) Top-down layout of Tripod. This was to pull Macrura up and slightly forward to prevent damaging bottom sensors.

Figure A-1: Diagrams used to brief the recovery team on the plan. Helped convey the importance of the spacing between holes and positioning of the tripod during vehicle removal.

\section{A.1 Recovery Images}




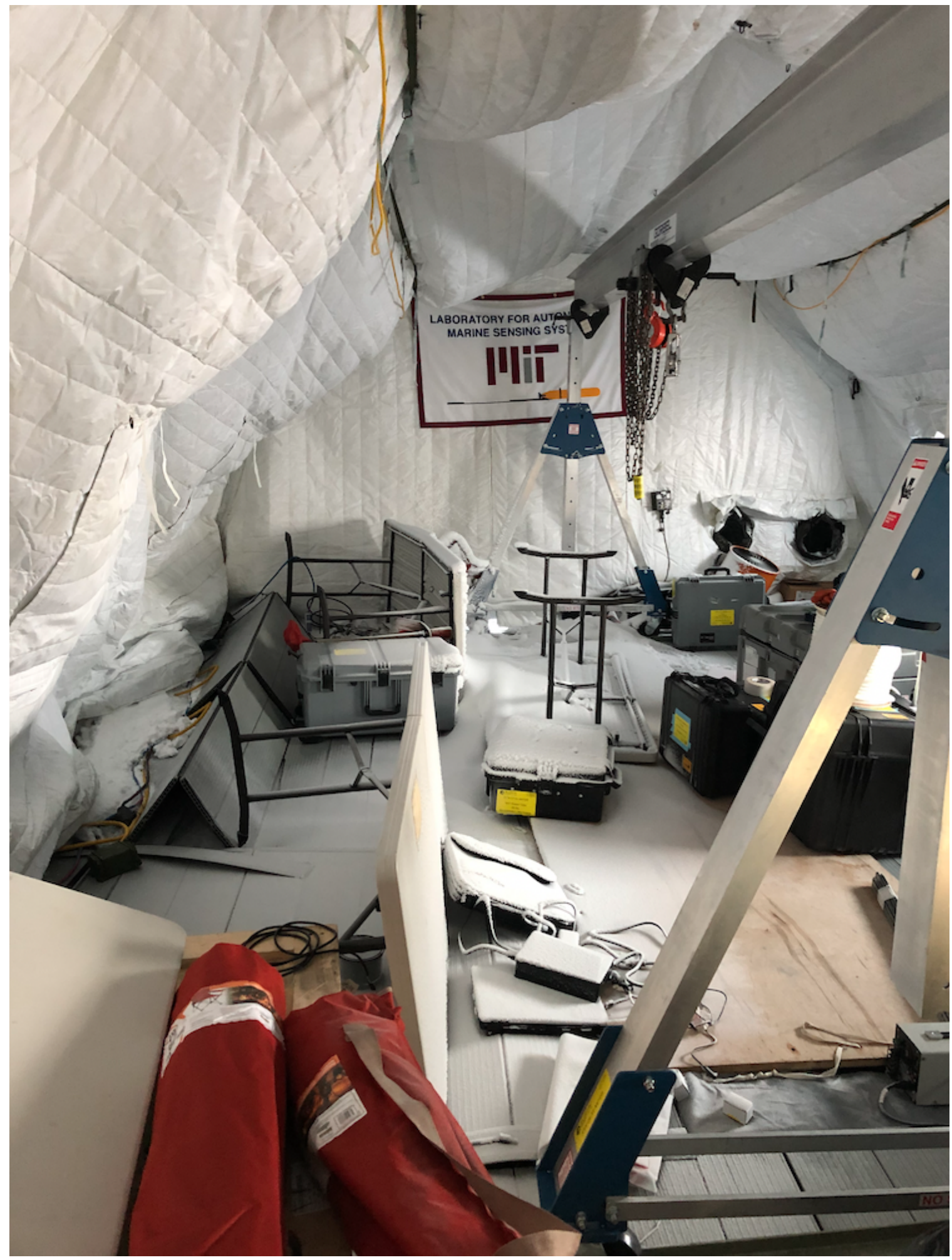

Figure A-2: Returning to the ice camp following the snowstorm. The operations tent and the hydrohole were in need of serious repair. 


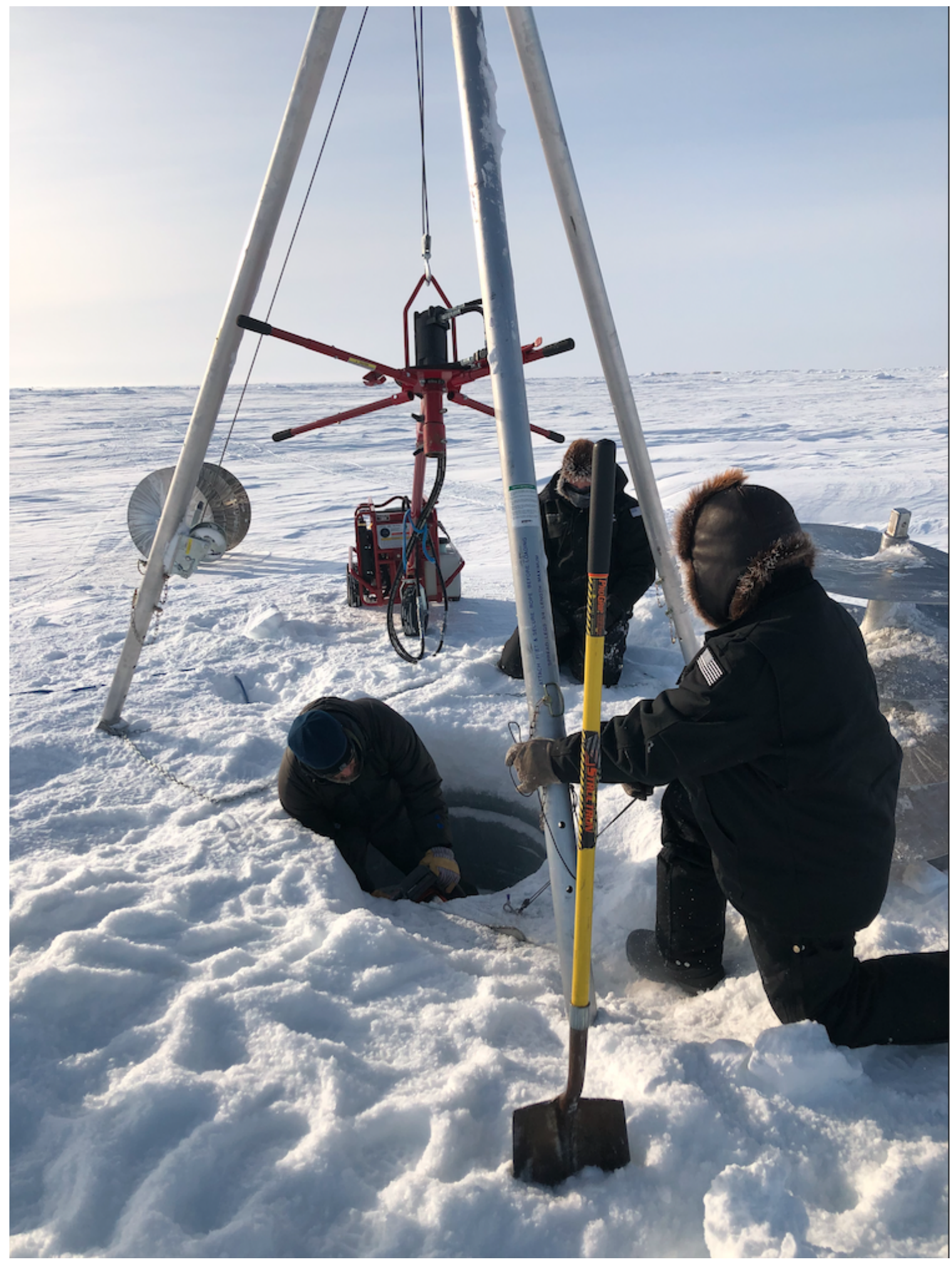

Figure A-3: After digging the first hole, without breaking through the ice, an ASL operator is inside the hole using a chainsaw to extend the upper corner. Since the two holes end to end were only 64" long. This additional length at the top was intended for the nose of the vehicle to go into. It would reduce the angle that the vehicle was going to undergo during recovery. The ice anchor is present to prevent the ice chunk from falling into the hole where the operator is. 


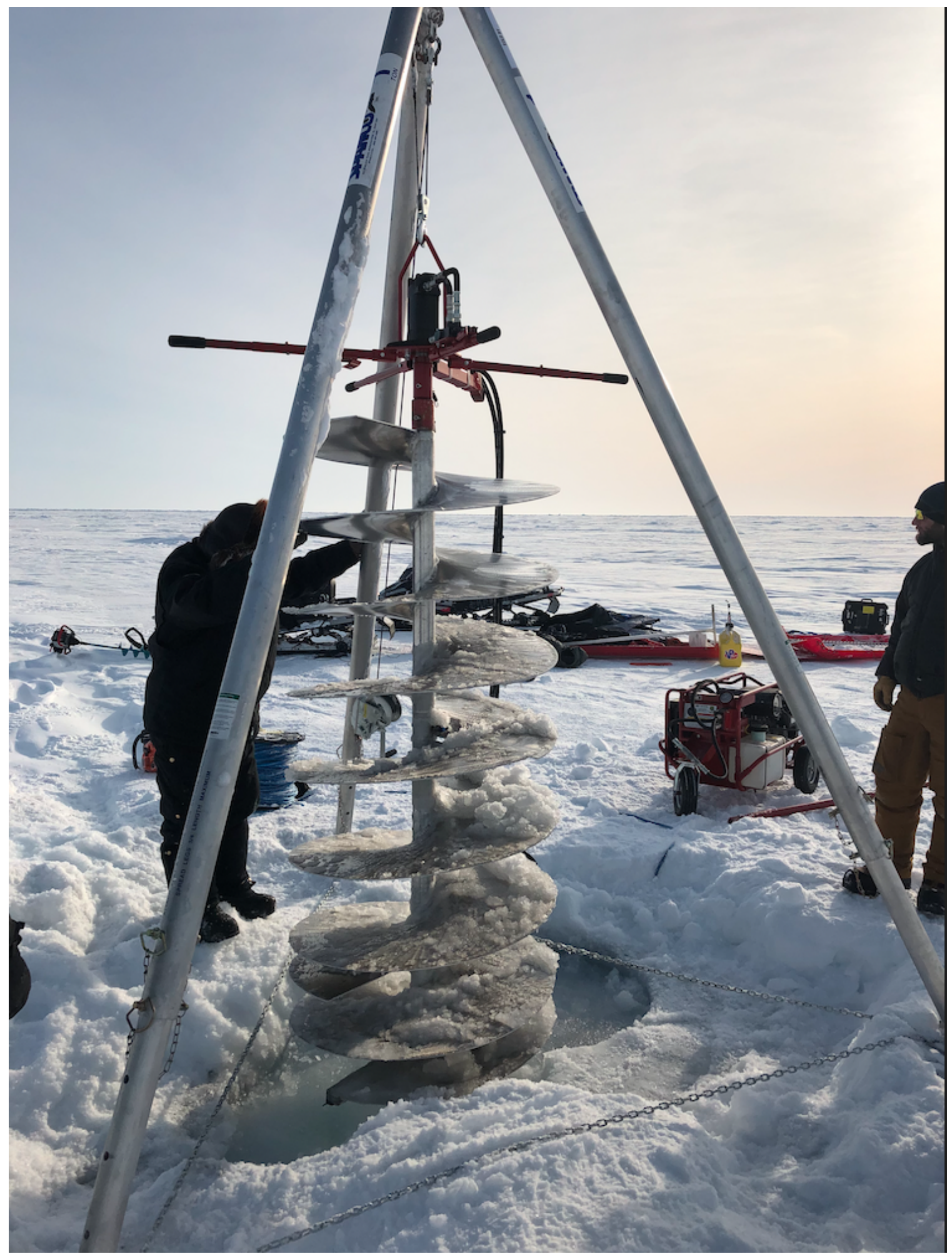

Figure A-4: This is the 36" auger that was used for drilling out the two holes. We decided on two based on the expertise from ASL. From their years of experience on the ice, it was clear that doing 2-holes with the first dry was achievable, but to attempt to do more holes (3 or 4 ) without breaking through the ice is extremely challenging. 


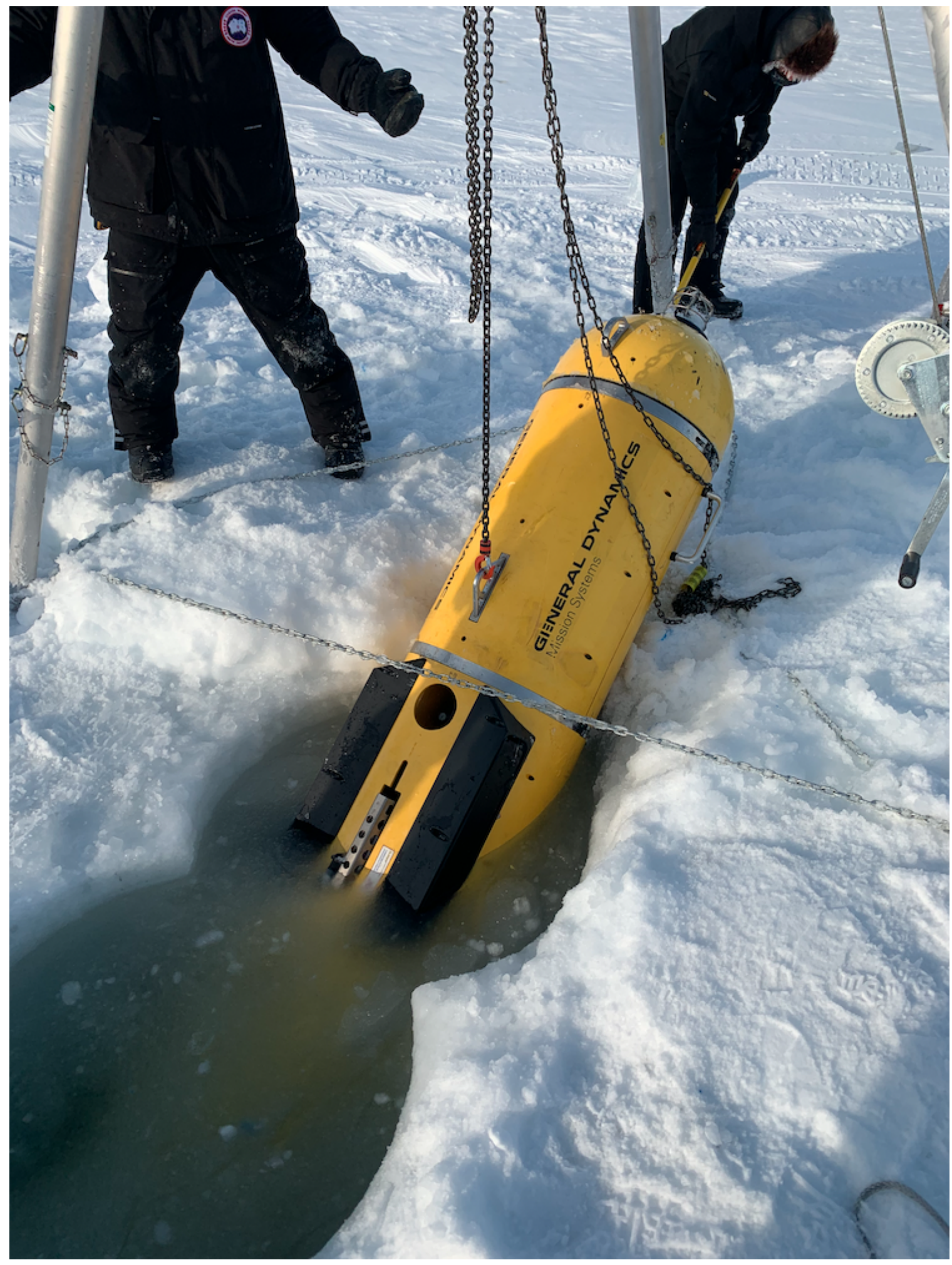

Figure A-5: Macrura being removed from the recovery hole. The two holes were separated by 28 ". This provided just over $22 "$ at the narrowest point for the vehicle to fit through. The vehicle is $21^{\prime \prime}$ in diameter. This distance was to maximize the length of the two holes while ensuring the vehicle would fit through the narrowest part. 


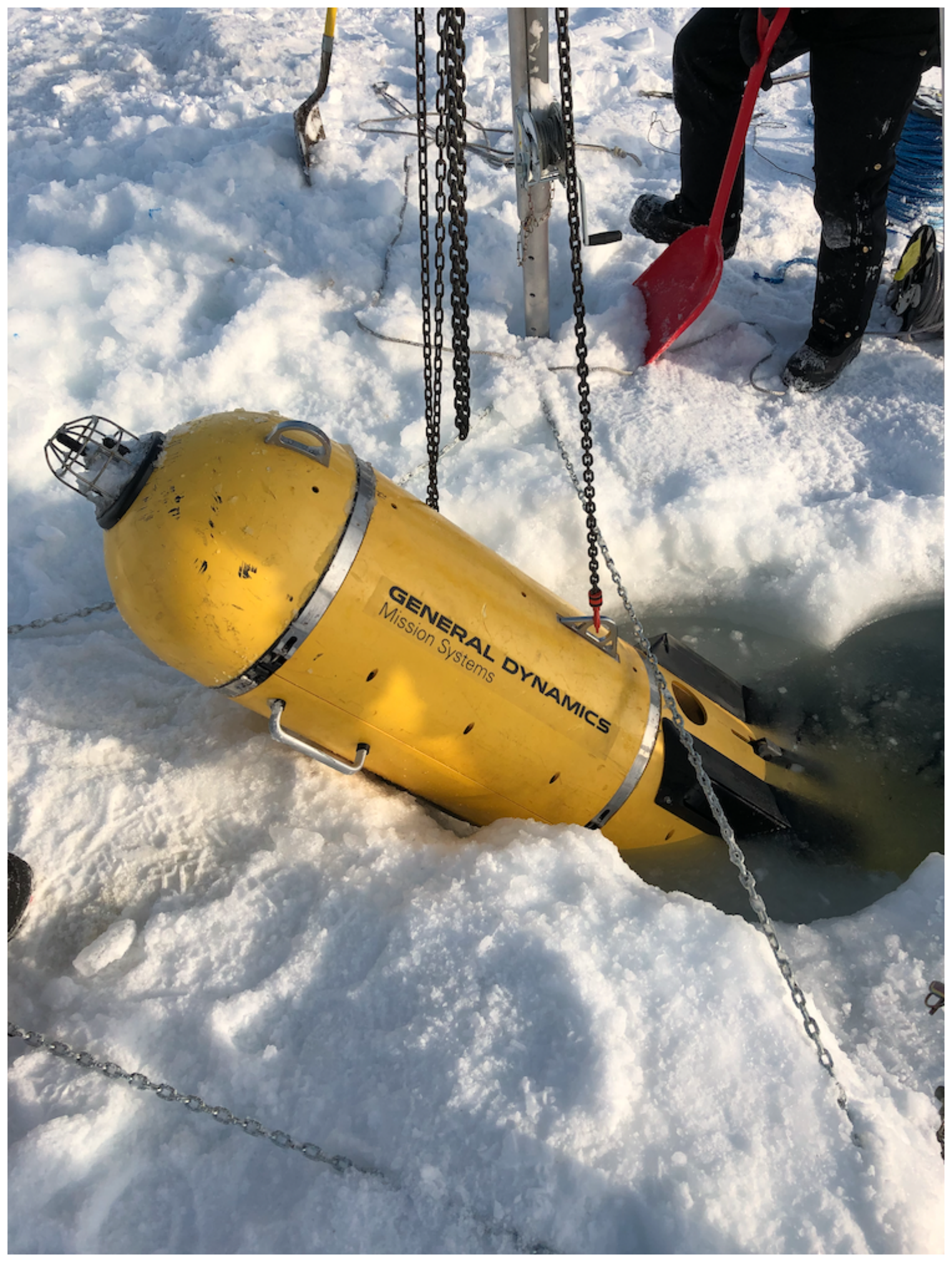

Figure A-6: The chain fall is connected to the FWD weight bearing mount on the vehicle. The nose handle and the side handle were not used to extract the vehicle. Those handles are not weight bearing mounts. 


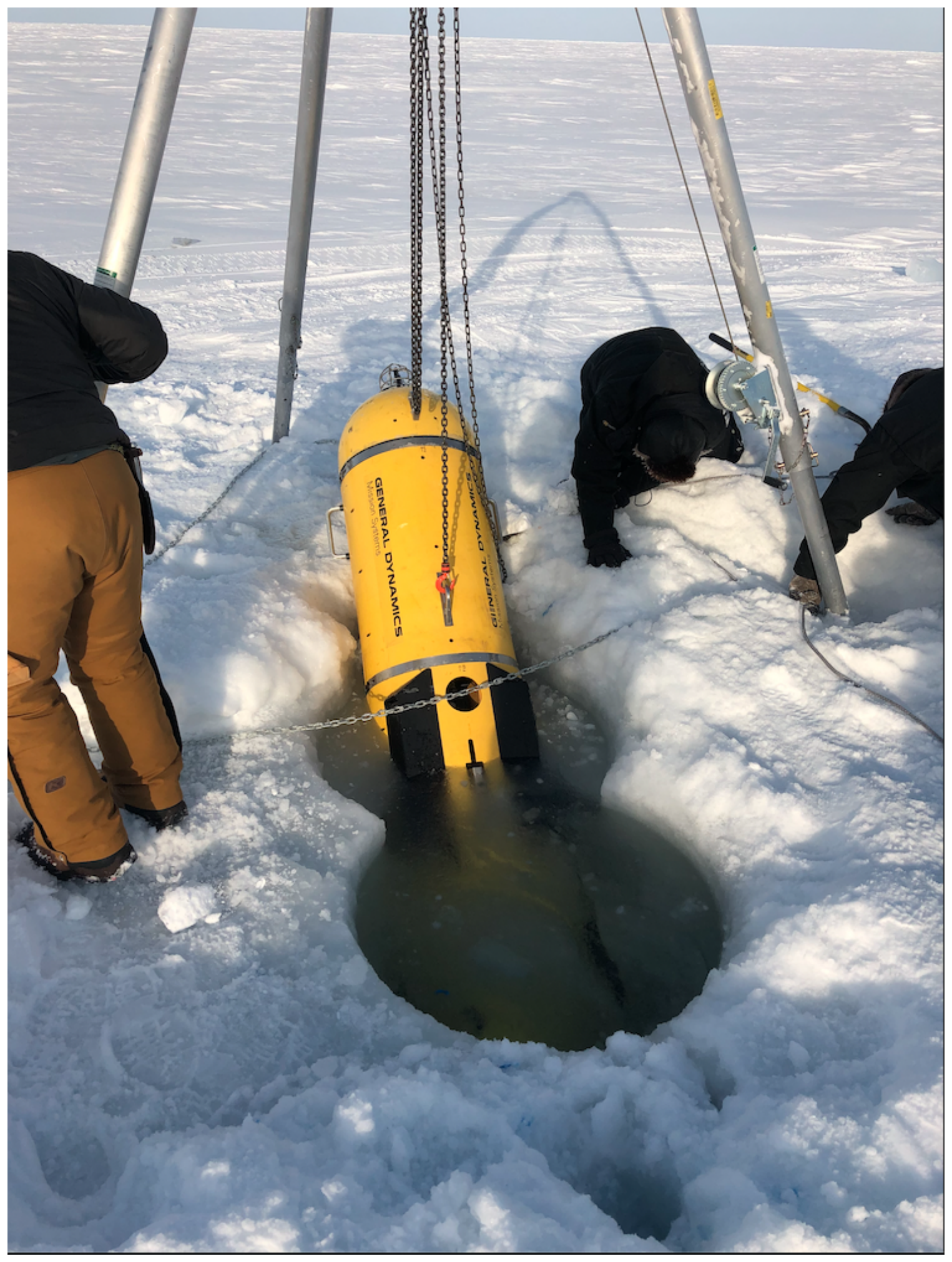

Figure A-7: This helps show just how tight it was for the vehicle to squeeze through the narrowest part of the two holes. As well, the slot cut in the front of the holes with the chainsaw was critical in reducing the angle of removal on the vehicle. The angle was roughly $50^{\circ}$ 


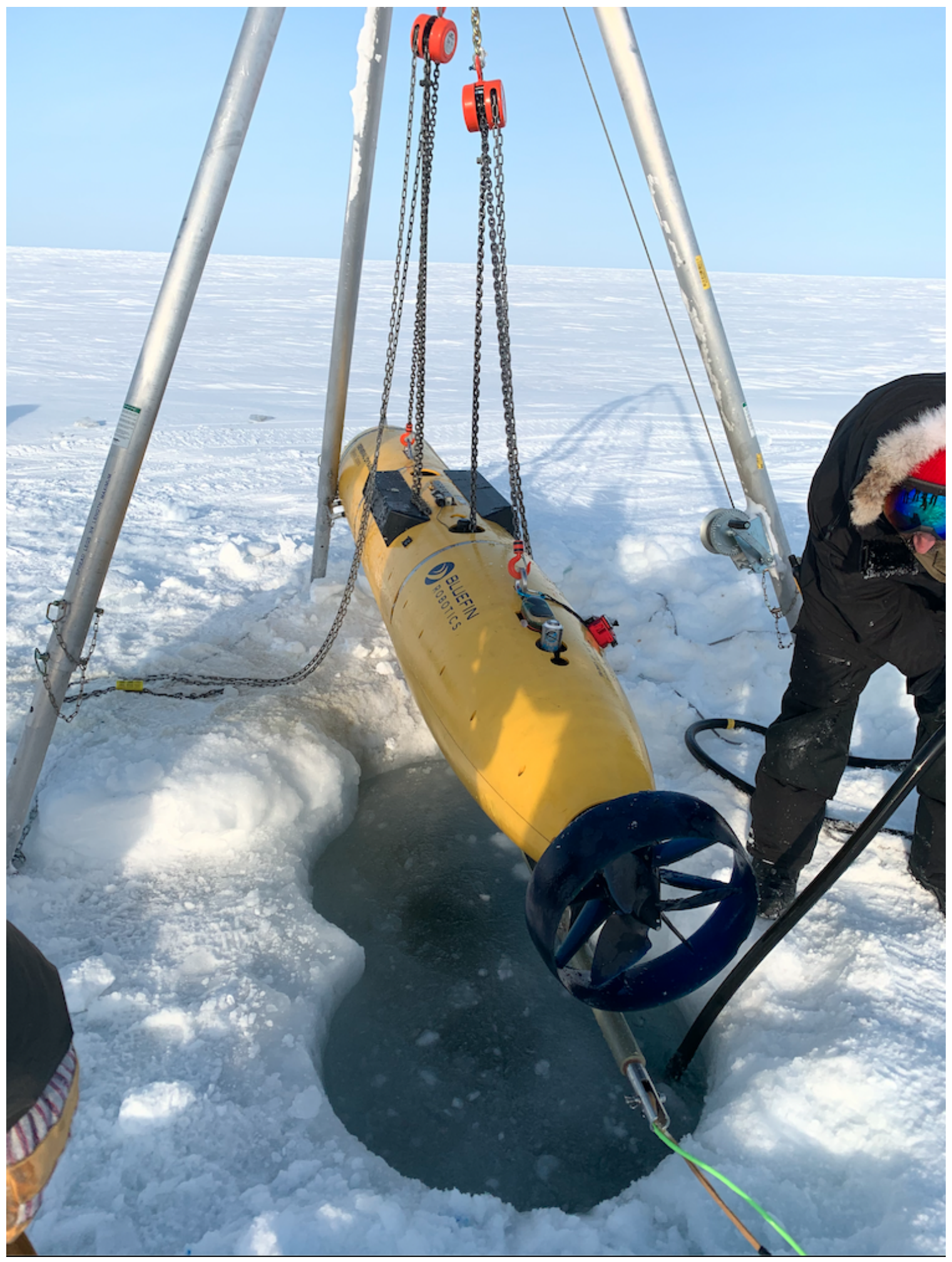

Figure A-8: Macrura is fully out of the water and the DURIP towed array is being removed from the water and disconnected. 


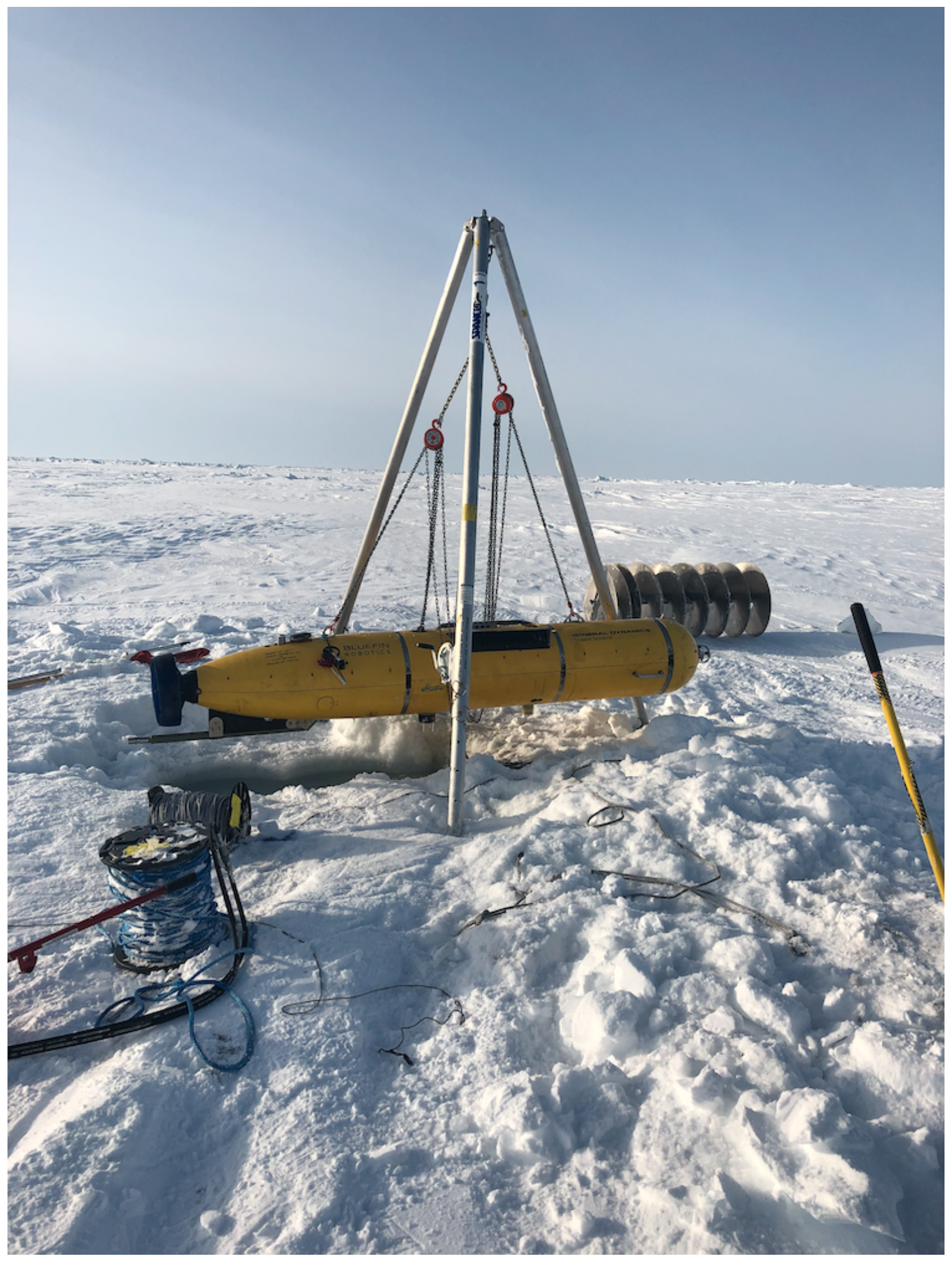

Figure A-9: The vehicle fully removed with both chain falls being used. One connected to the FWD mount and one to the AFT mount. The 36" auger is in the background. The two spools of cable used to recover the vehicle are on the bottom left. The blue line was used to tie off the vehicle to topside through the duration of the snowstorm. 


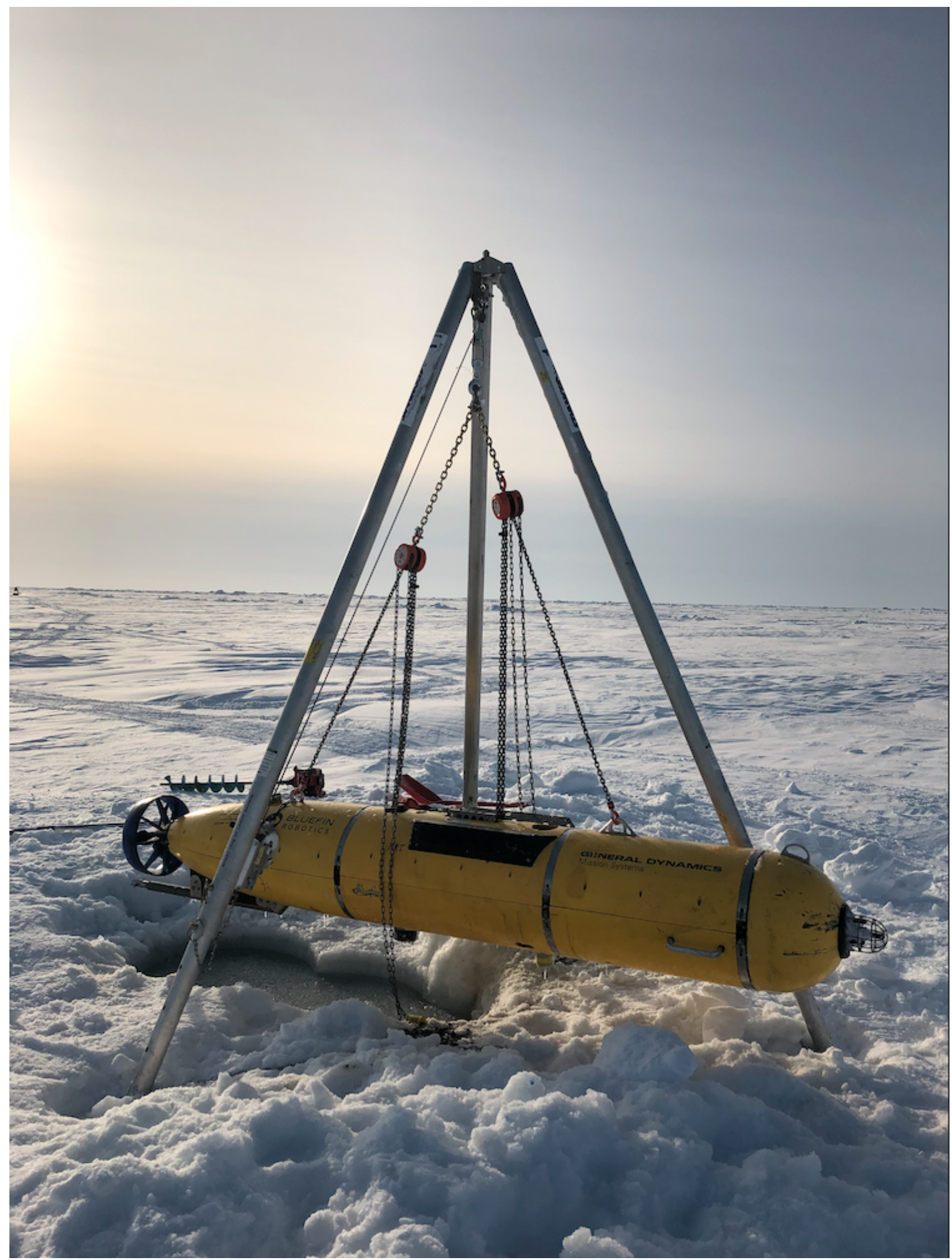

Figure A-10: Macrura fully removed from the water awaiting transit back to ice camp. 


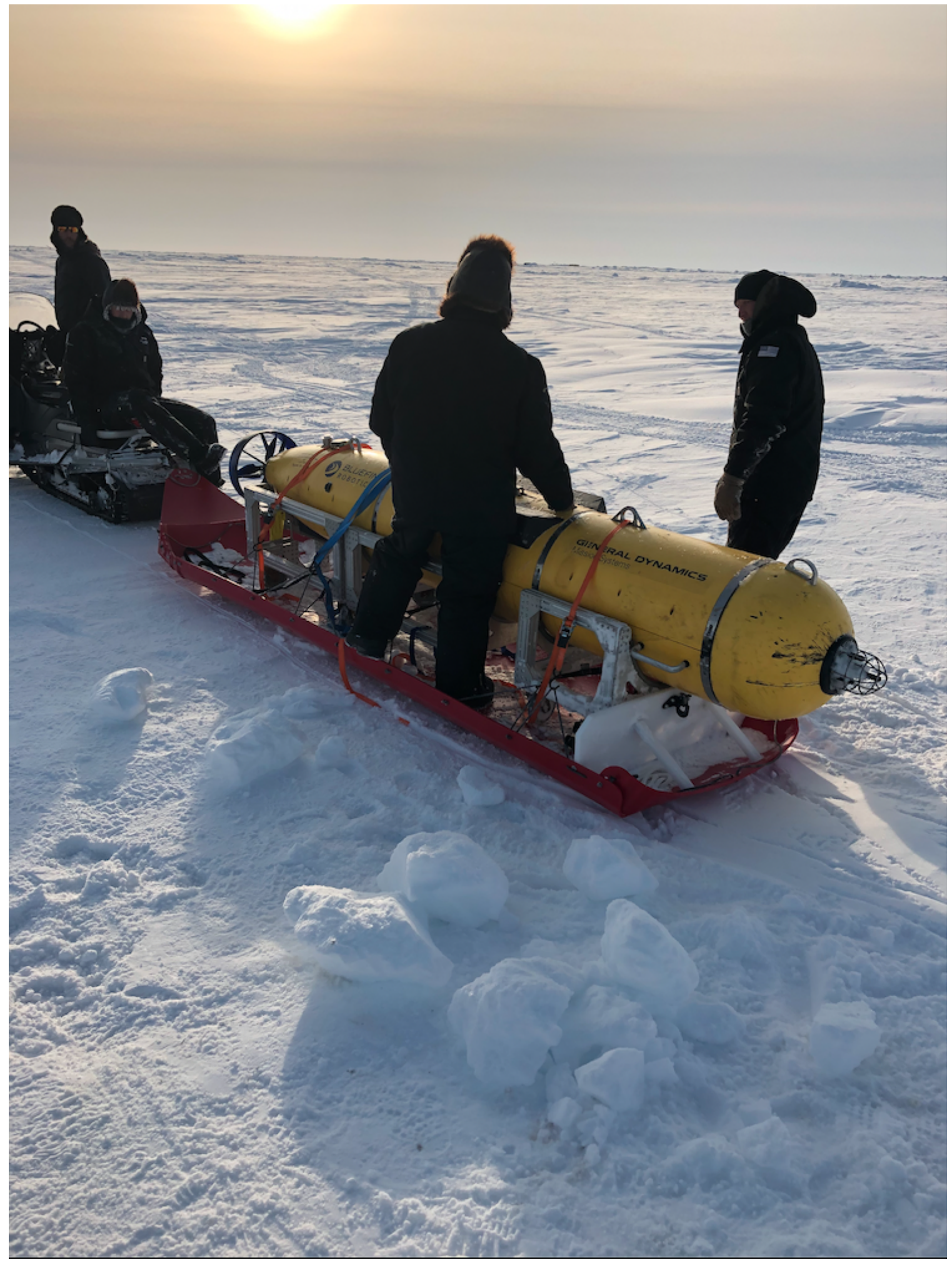

Figure A-11: The vehicle was tied off to a snow sled and towed behind a snowmobile back to ice camp for disassembly and transit back to Prudhoe Bay, AK. 
THIS PAGE INTENTIONALLY LEFT BLANK 


\section{Appendix B}

\section{DURIP Towed Array Beam Patterns}

Since the beamforming on the DURIP towed array didn't follow the conventional $d \leq \frac{\lambda}{2}$, this set of plots help visualize and understand how the beam patterns of the array change as we increased frequency up to $6 \mathrm{kHz}$, which is a factor of 6 greater than the nested array max frequency of $1 \mathrm{kHz}$. As well, the target frequency to beamform from was a $5 \mathrm{kHz}$ signal, the subharmonic of the actual $10 \mathrm{kHz}$ transmitted signal for the WHOI micro-modem buoys.

\section{B.1 23 Element Polar Beam Patterns}

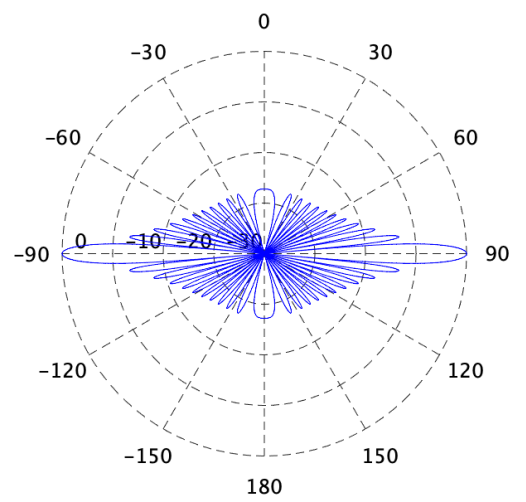

(a) $d=\frac{\lambda}{2}$

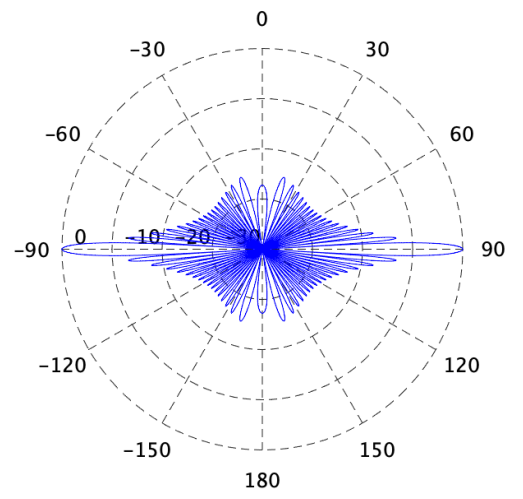

(b) $d=\frac{1.5 \lambda}{2}$

Figure B-1: Polar plots of 23 element nested array with $d=\frac{1-1.5 \lambda}{2}$ 


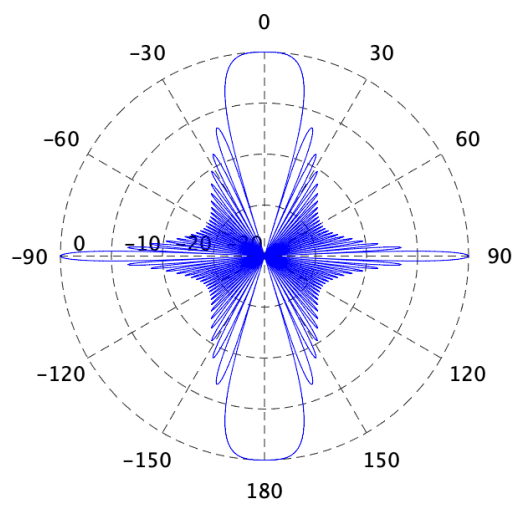

(a) $d=\frac{2 \lambda}{2}$

23 Elements Uniform Weight with Steering Angle $=90 ; d=3 \lambda / 2$

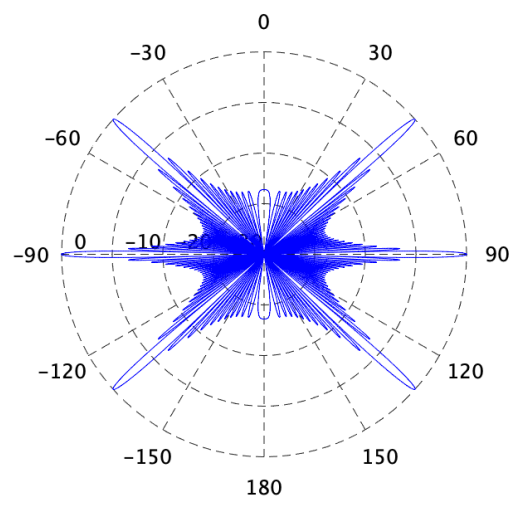

(c) $d=\frac{3 \lambda}{2}$

23 Elements Uniform Weight with Steering Angle $=90 ; d=4 \lambda / 2$

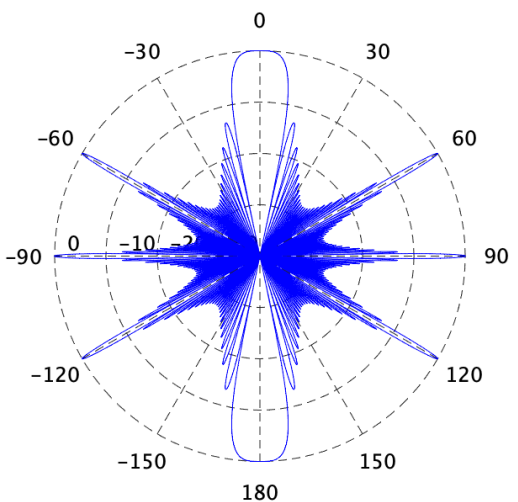

(e) $d=\frac{4 \lambda}{2}$

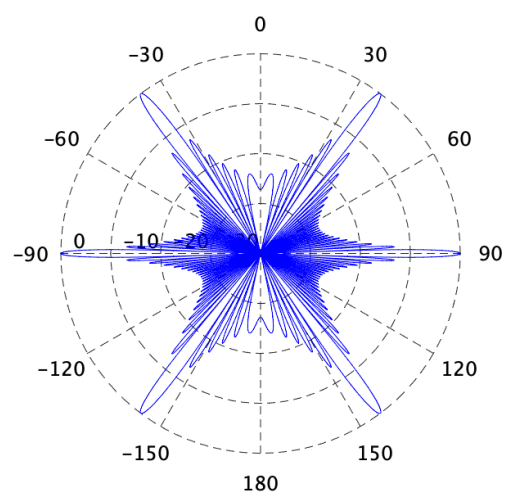

(b) $d=\frac{2.5 \lambda}{2}$

23 Elements Uniform Weight with Steering Angle $=90 ; \mathrm{d}=3.5 \lambda / 2$

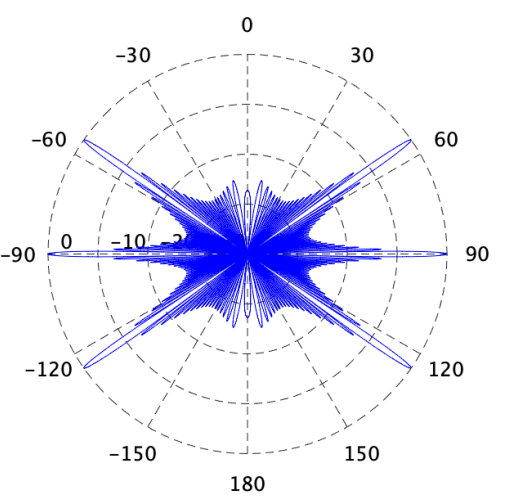

(d) $d=\frac{3.5 \lambda}{2}$

23 Elements Uniform Weight with Steering Angle $=90 ; d=4.5 \lambda / 2$

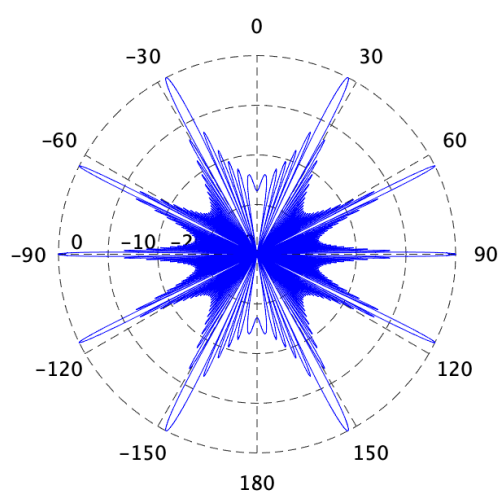

(f) $d=\frac{4.5 \lambda}{2}$

Figure B-2: Polar plots of 23 element nested array with $d=\frac{2-4.5 \lambda}{2}$ 


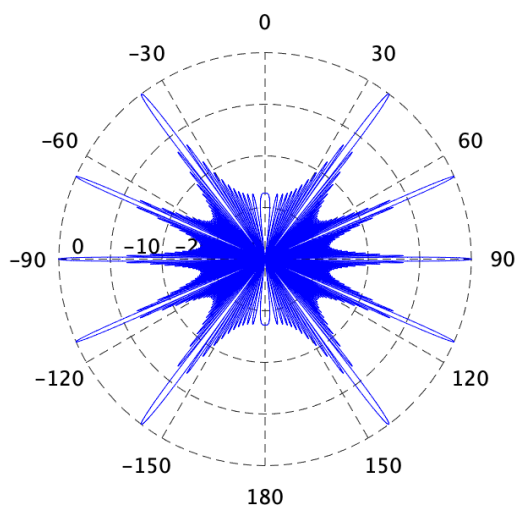

(a) $d=\frac{5 \lambda}{2}$

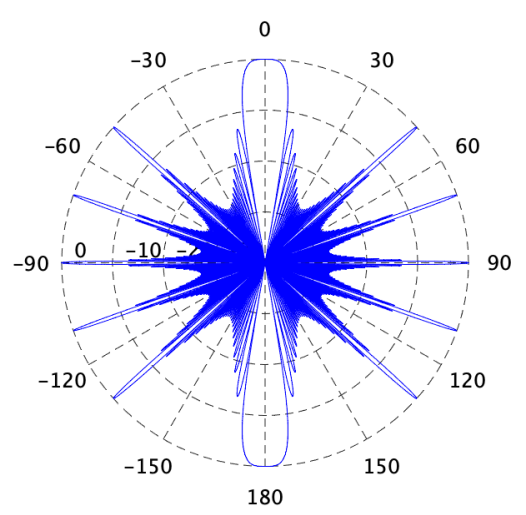

(b) $d=\frac{6 \lambda}{2}$

Figure B-3: Polar plots of 23 element nested array with $d=\frac{5-6 \lambda}{2}$

\section{B.2 23 Element u-space Beam Patterns}

In $\mathrm{u}$-space I've included out to $u= \pm 2$. This helps visualize the compression that occurs while we adjust $d$ as it relates to $\frac{\lambda}{2}$. Unlike in the polar plots, its much easier what to anticipate as the wavelength gets smaller since you can see outside the visual region.

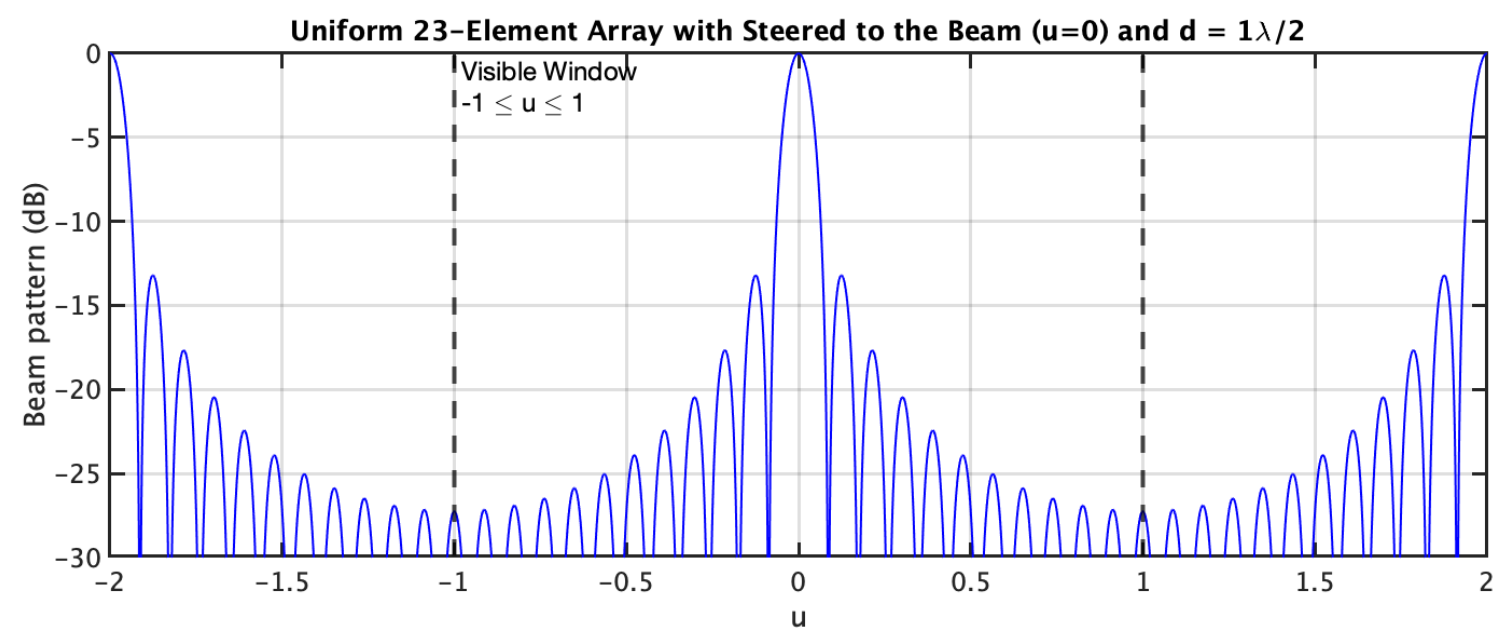

Figure B-4: 23 Element nested array plotted in u-space with $d=\frac{\lambda}{2}$ 


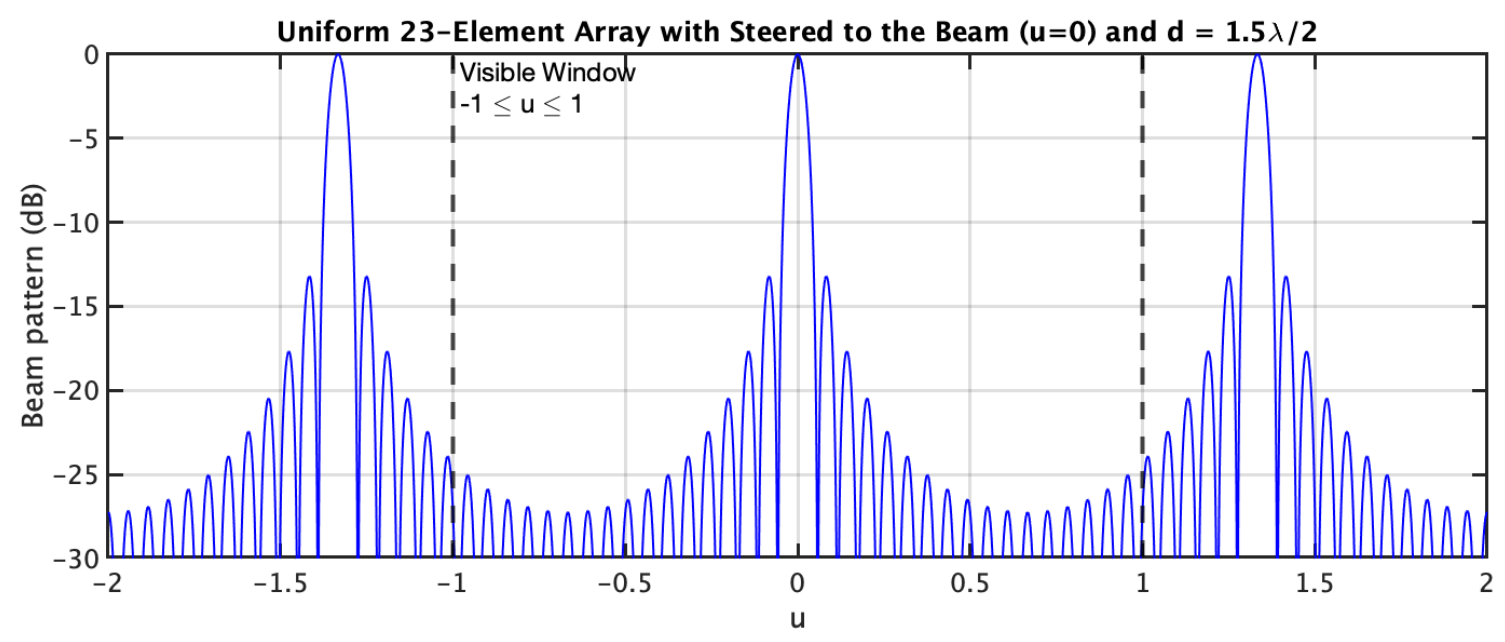

(a) 23 Element nested array plotted in u-space with $d=\frac{1.5 \lambda}{2}$

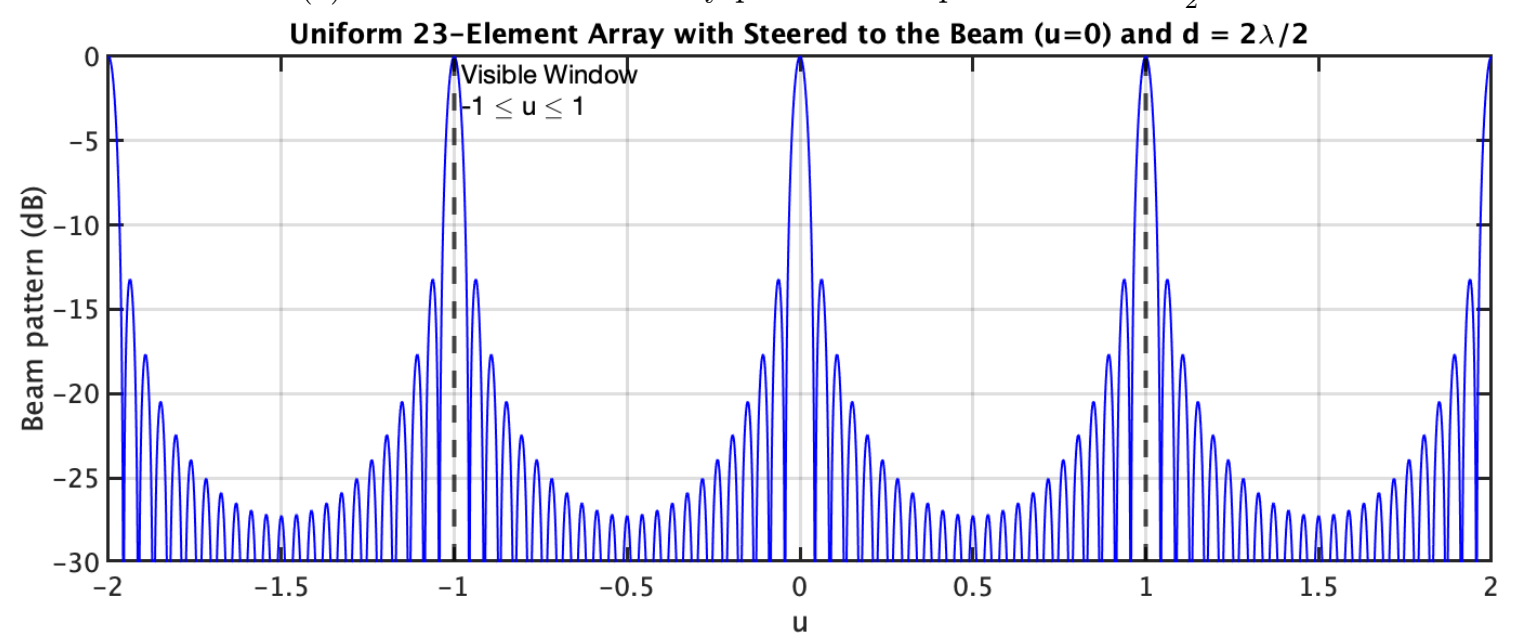

(b) 23 Element nested array plotted in u-space with $d=\frac{2 \lambda}{2}$

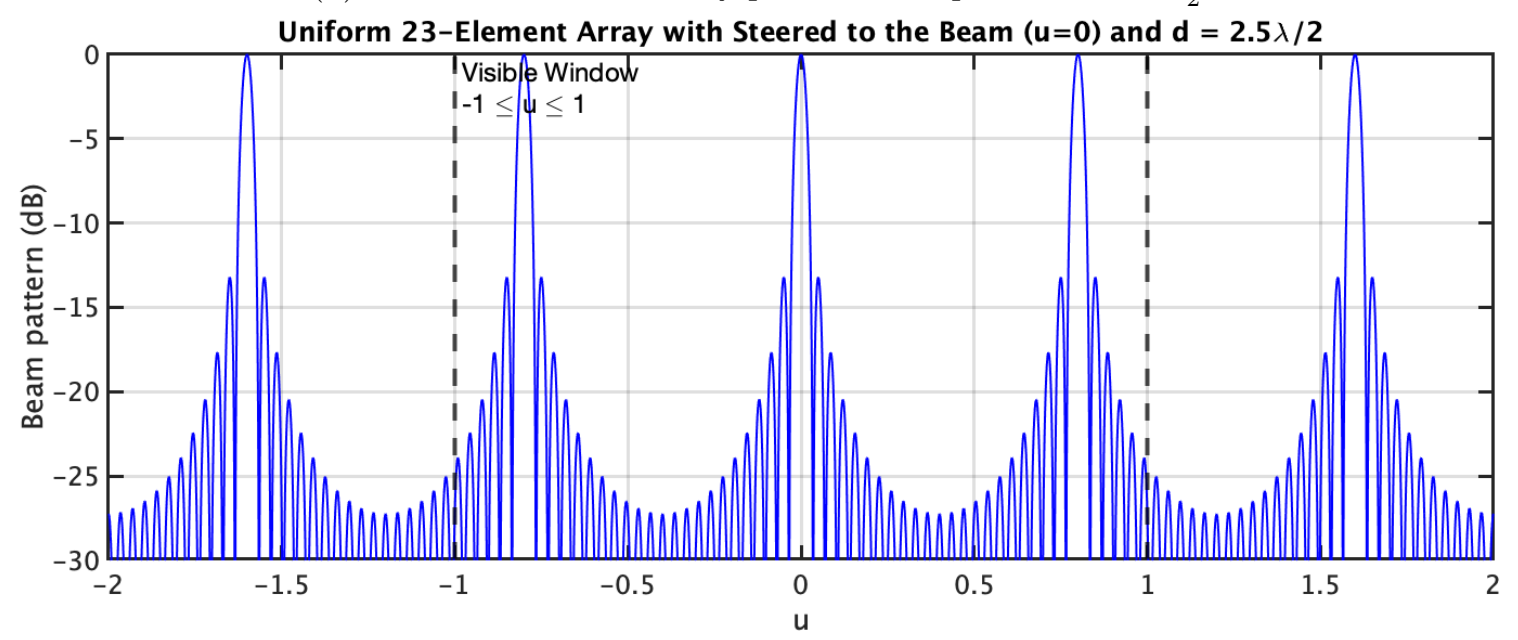

(c) 23 Element nested array plotted in u-space with $d=\frac{2.5 \lambda}{2}$

Figure B-5: 23 Element nested array in u-space with $d=\frac{1.5-2.5 \lambda}{2}$ 


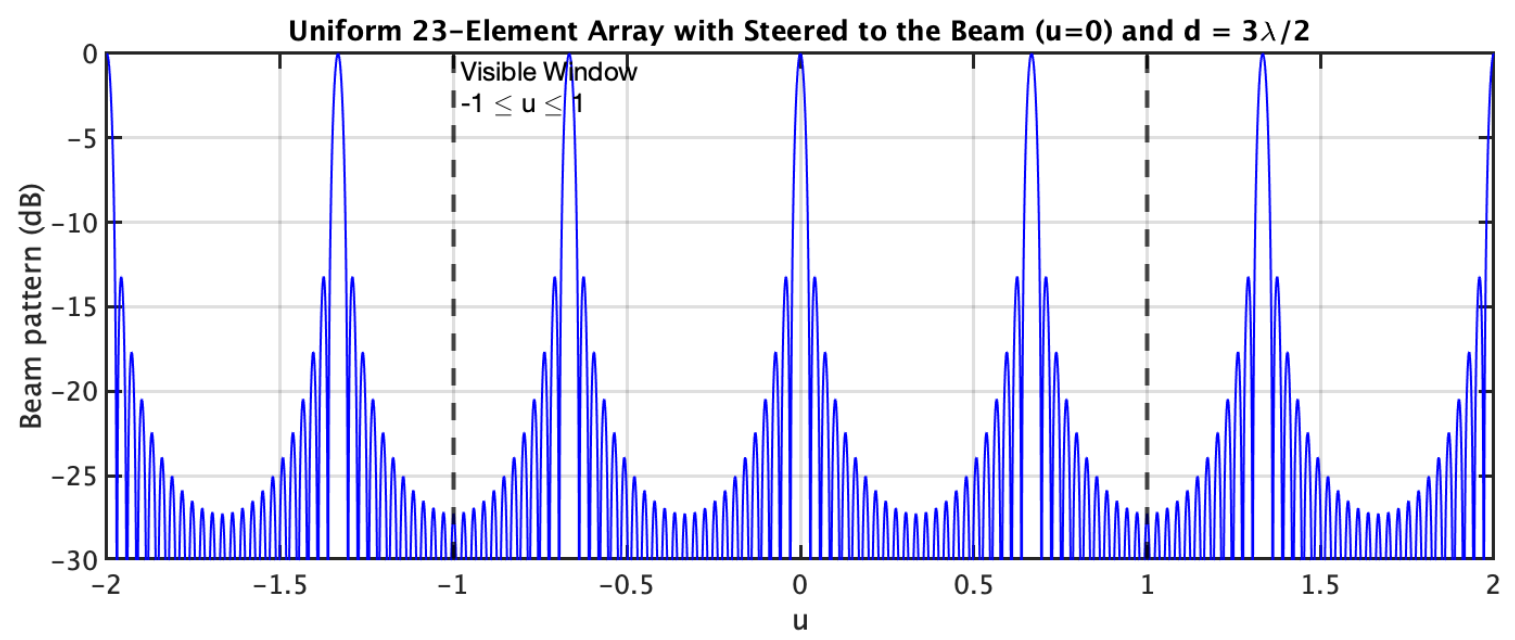

(a) 23 Element nested array plotted in u-space with $d=3 \frac{\lambda}{2}$

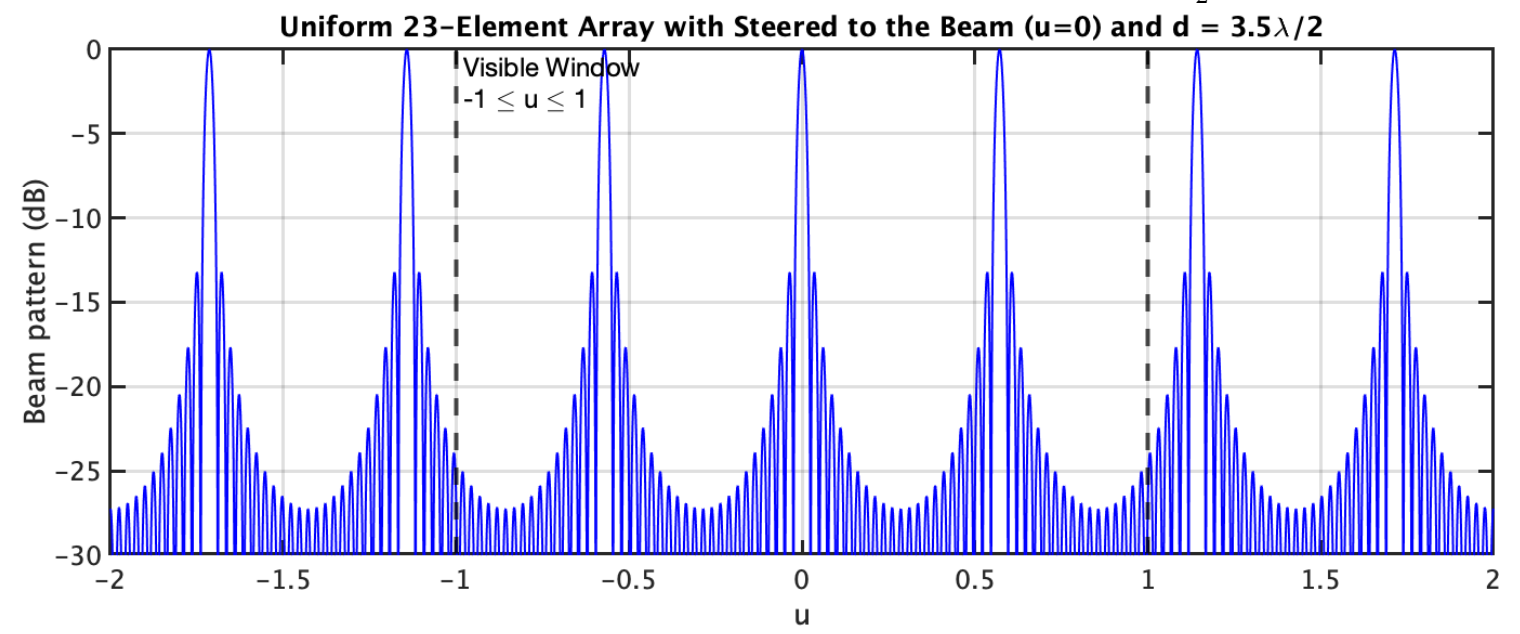

(b) 23 Element nested array plotted in u-space with $d=\frac{3.5 \lambda}{2}$

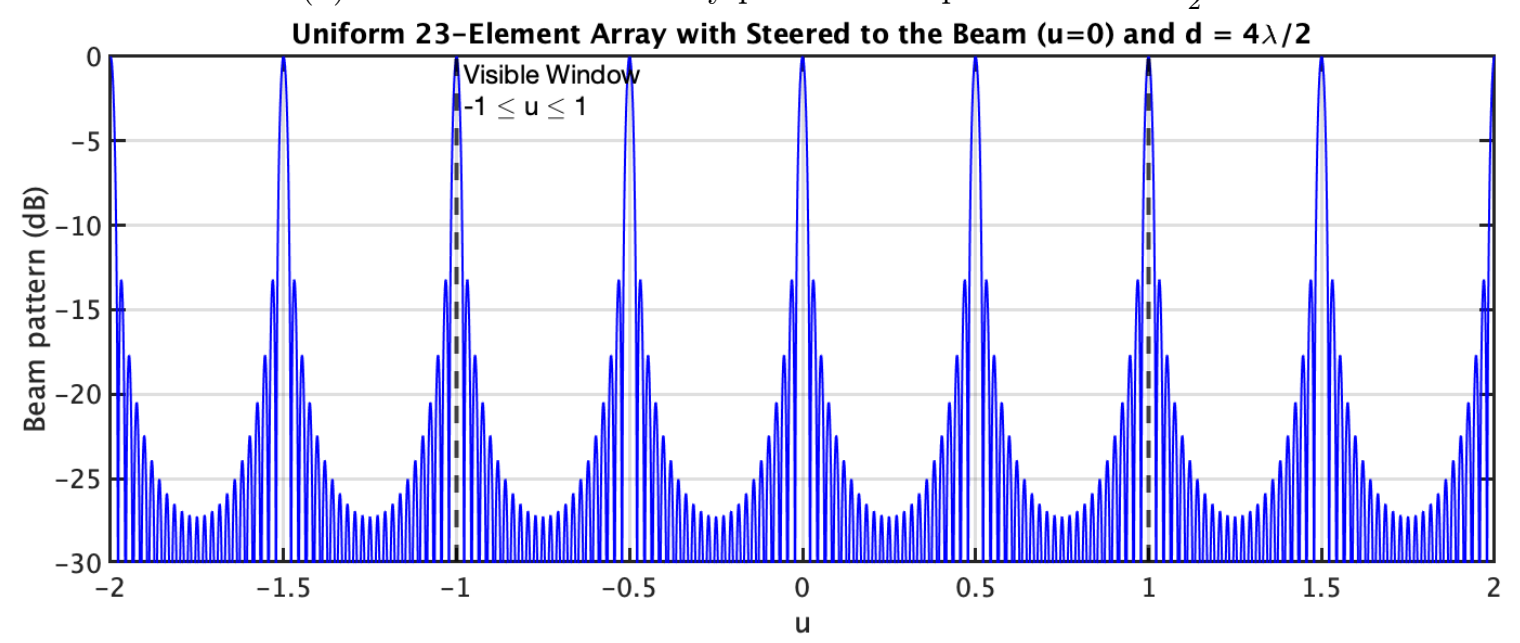

(c) 23 Element nested array plotted in u-space with $d=\frac{4 \lambda}{2}$

Figure B-6: 23 Element nested array in u-space with $d=\frac{3-4 \lambda}{2}$ 


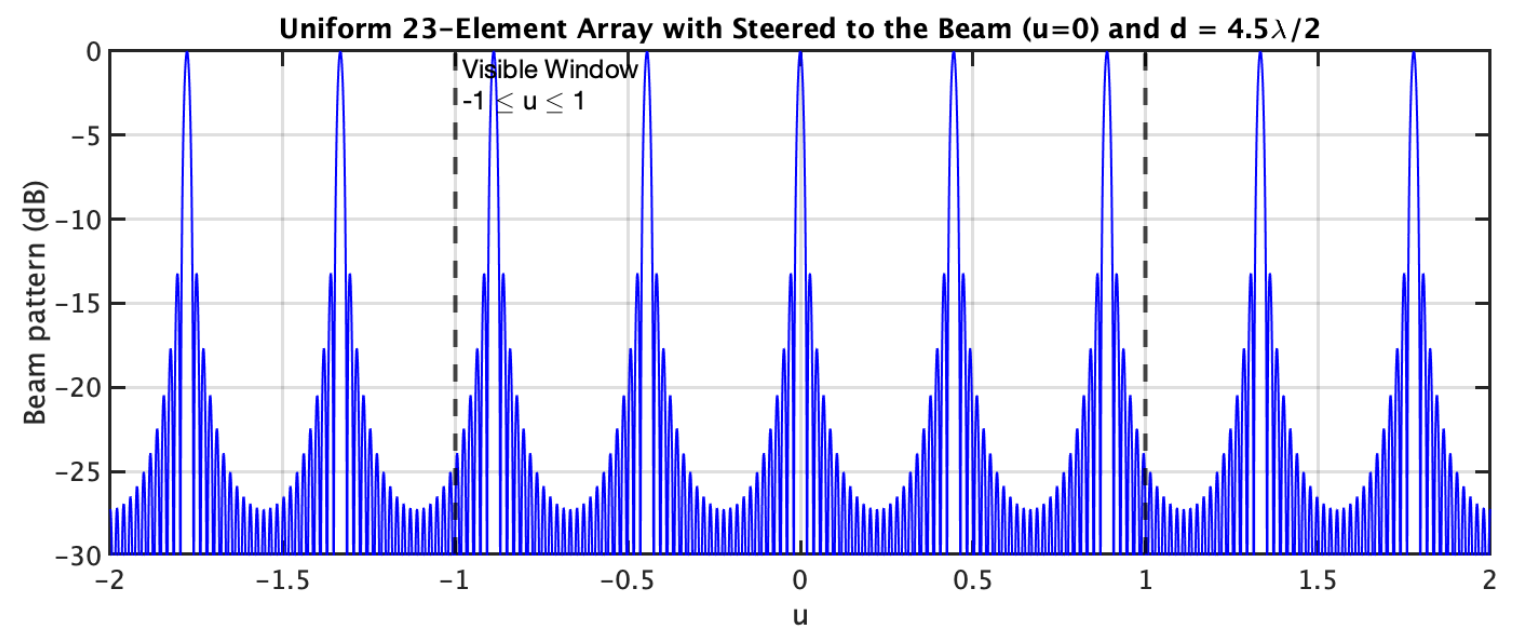

(a) 23 Element nested array plotted in u-space with $d=\frac{4.5 \lambda}{2}$

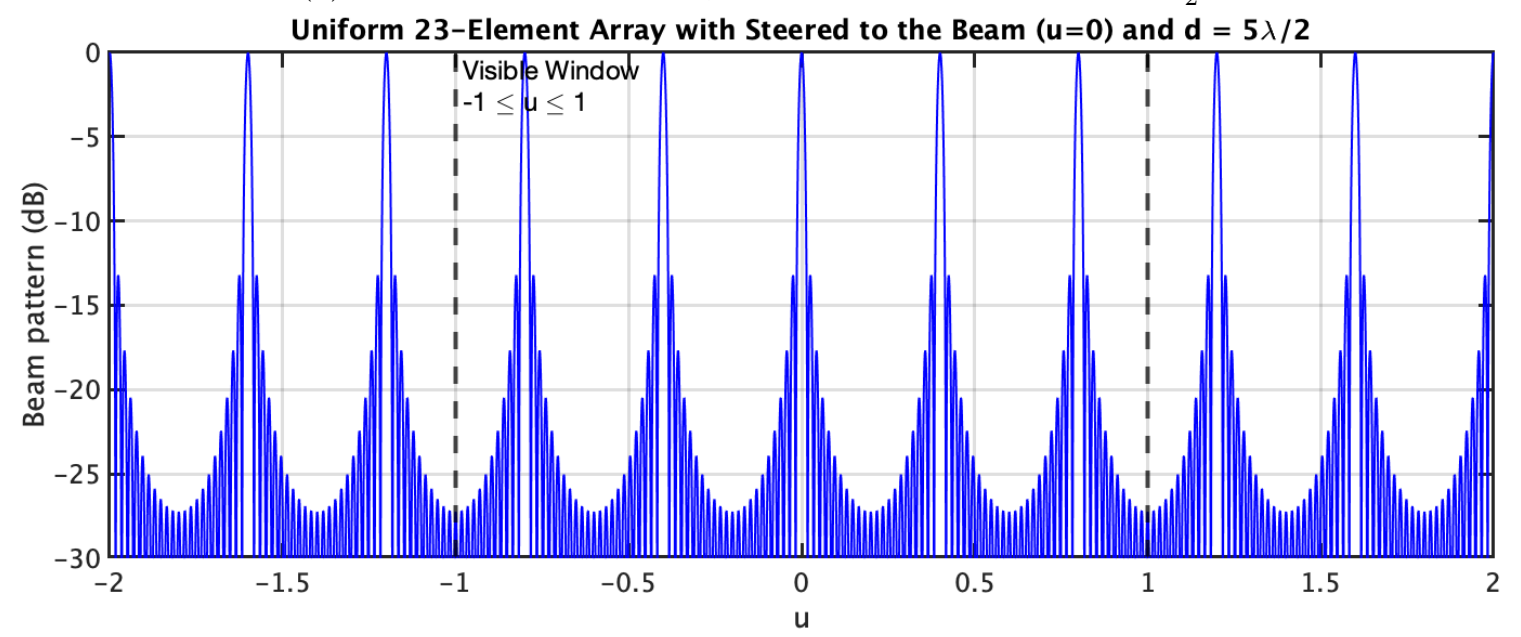

(b) 23 Element nested array plotted in u-space with $d=\frac{5 \lambda}{2}$

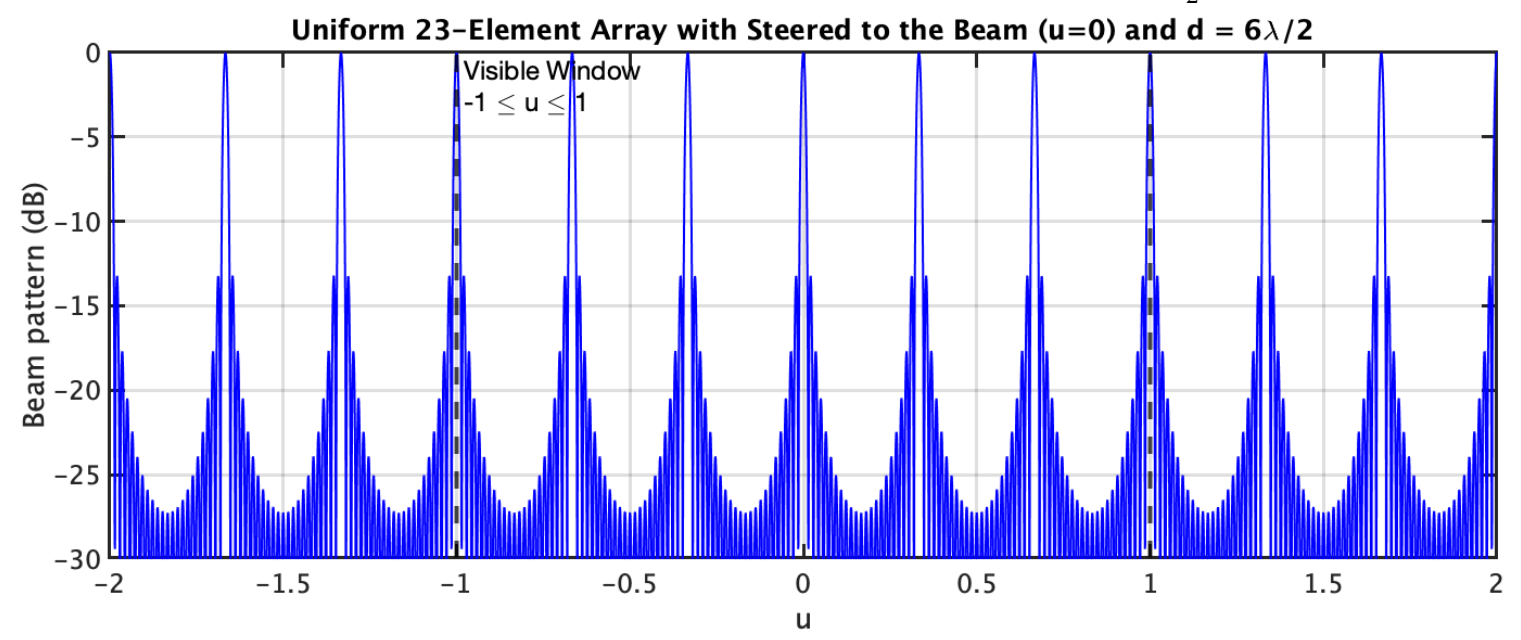

(c) 23 Element nested array plotted in u-space with $d=\frac{6 \lambda}{2}$

Figure B-7: 23 Element nested array in u-space with $d=\frac{4.5-6 \lambda}{2}$ 


\section{B.3 DURIP Towed Array Spatial Aliasing or Grating Reduc- tion}

In order to reduce the impacts of Spatial Aliasing, instead of focusing the Fourier Transform on a single particular frequency, if a larger basket of frequencies are selected, the Fast Fourier Transform (FFT) averages that data together. A signal that is aliased at a higher frequency may not necessarily show up aliased when looking at a larger window of frequencies and averaging them together. The following examples show how this happens for one of the transmissions that occurred during the Vertical Run.

The goal was to get the best fidelity around $5 \mathrm{kHz}$ without resulting in too much spatial aliasing that would potentially hide the signal of interest, or spatially alias the $5 \mathrm{kHz}$ signal of interest into other arrival angles that didn't actually represent the true arrival angle.

Following the example from a WHOI micro-modem transmission, there is an example from a transmission made from Macrura while the DURIP towed array was drooping vertically beneath the vehicle. As a result, the transmission from Macrura is expected to show up in FWD endfire of the array beamforming. 
B.3.1 Transmission $\# 1473, z_{s}=30 \mathrm{~m}$, DURIP towed array at $\sim 120 \mathrm{~m}$

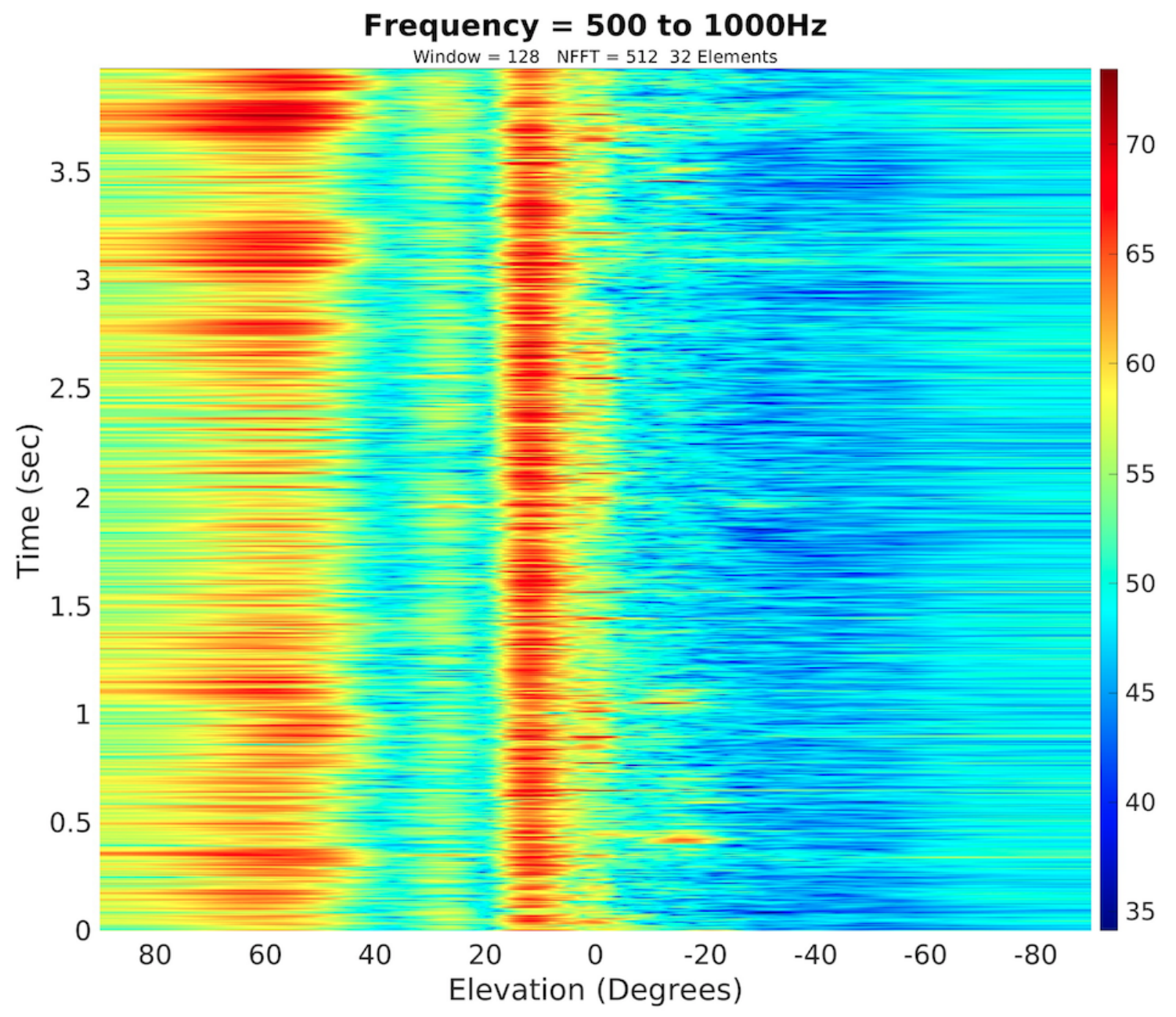

Figure B-8: DURIP Towed Array beamforming between $f=500-1000 \mathrm{~Hz}$, the designed spectrum for the array. The WHOI micro-modem transmission isn't visible in this spectrum, which makes sense considering the PSD in Figure 1-13. All of the energy from the WHOI micro-modem transmissions occur between 5-6 kHz. 


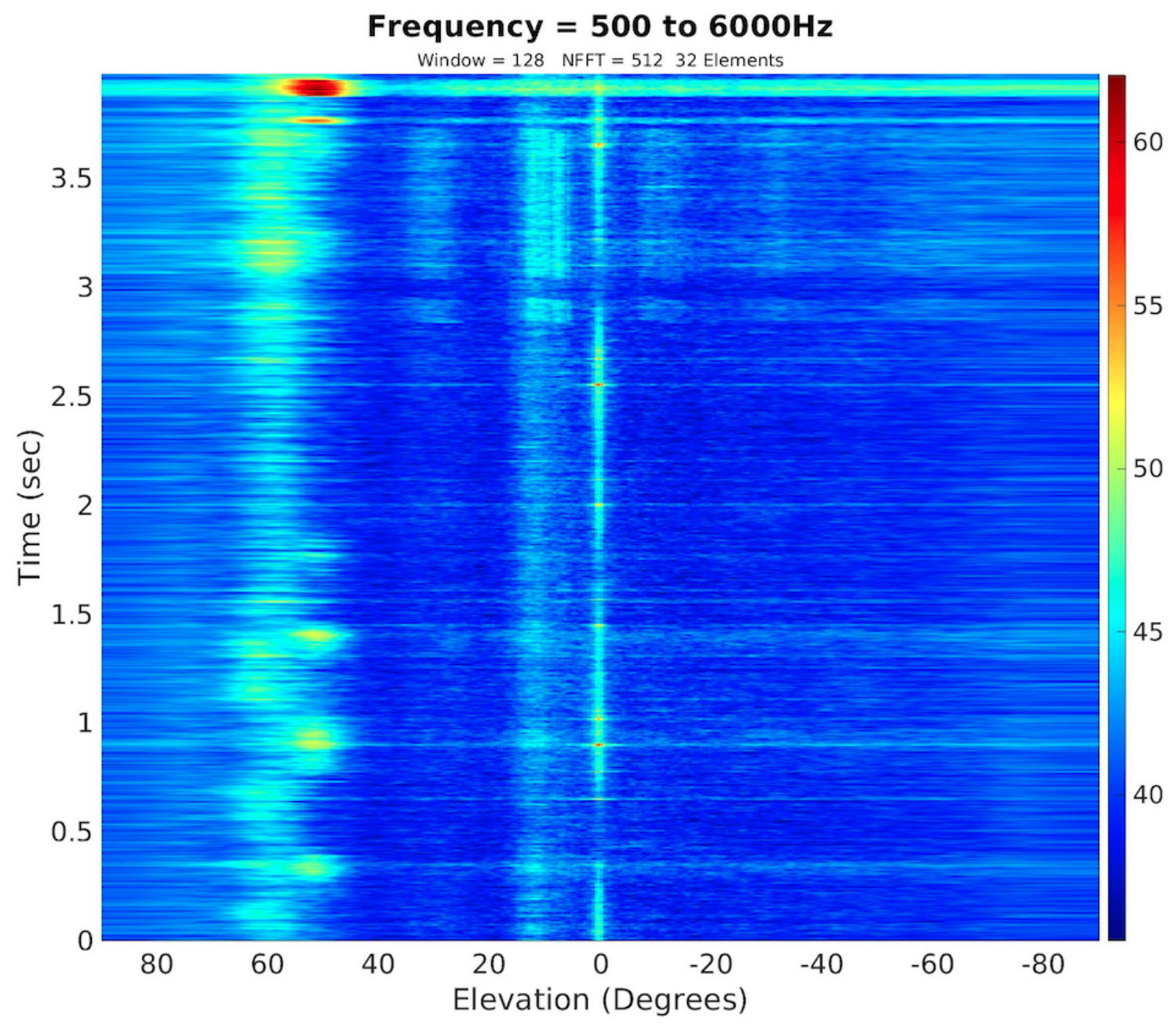

Figure B-9: DURIP Towed Array beamforming between $f=500-6000 H z$. Although portions of this are in the frequency spectrum where grating lobes are present, it's a dramatic change from Figure B-8, where the WHOI micro-modem transmission isn't even visible. 


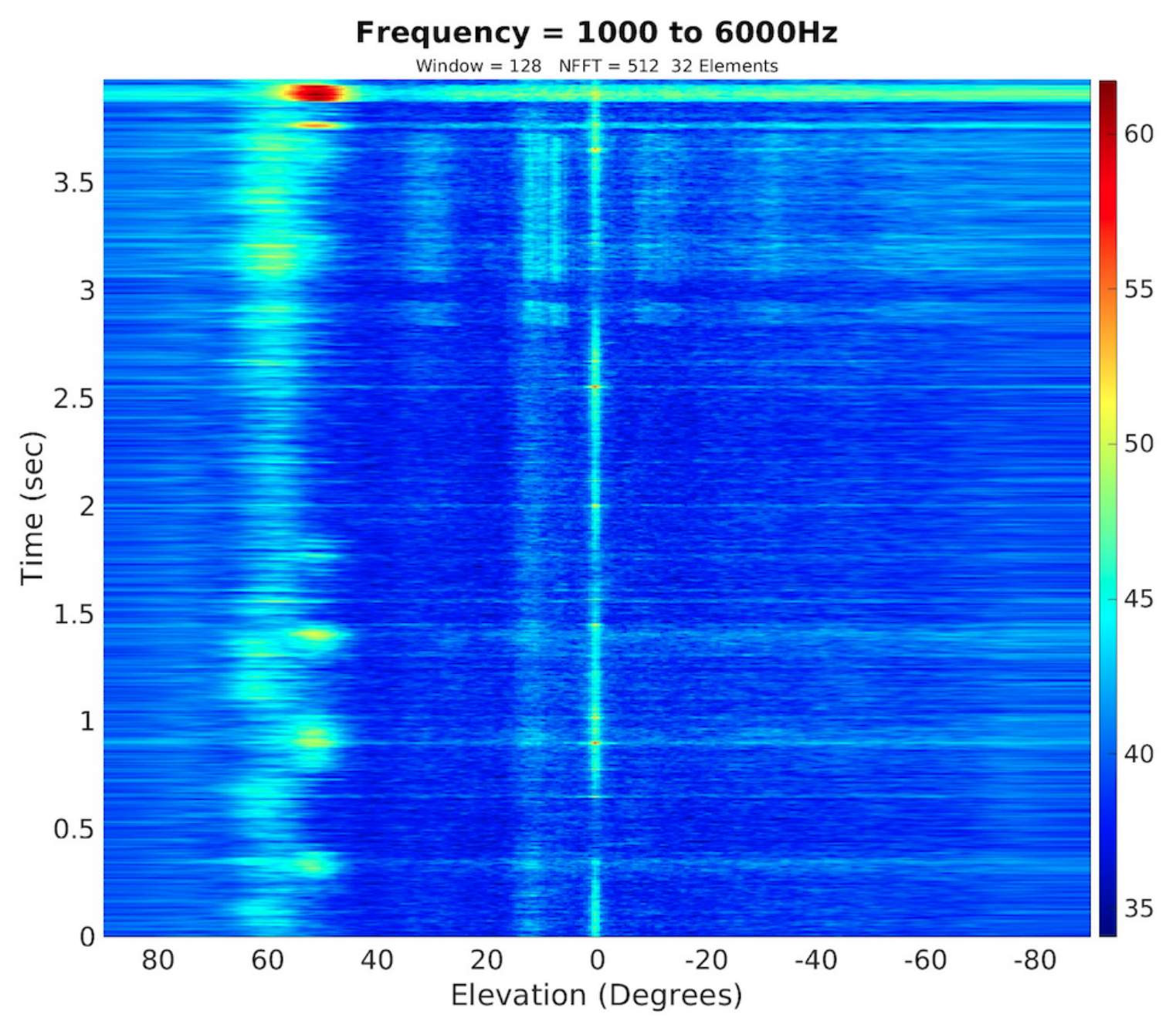

Figure B-10: DURIP Towed Array beamforming between $f=1000-6000 \mathrm{~Hz}$. Since $f \geq$ $1000 \mathrm{~Hz}$ this includes spatial aliasing, but the impacts are limited due to the large group of frequencies that beamforming is performed over. 


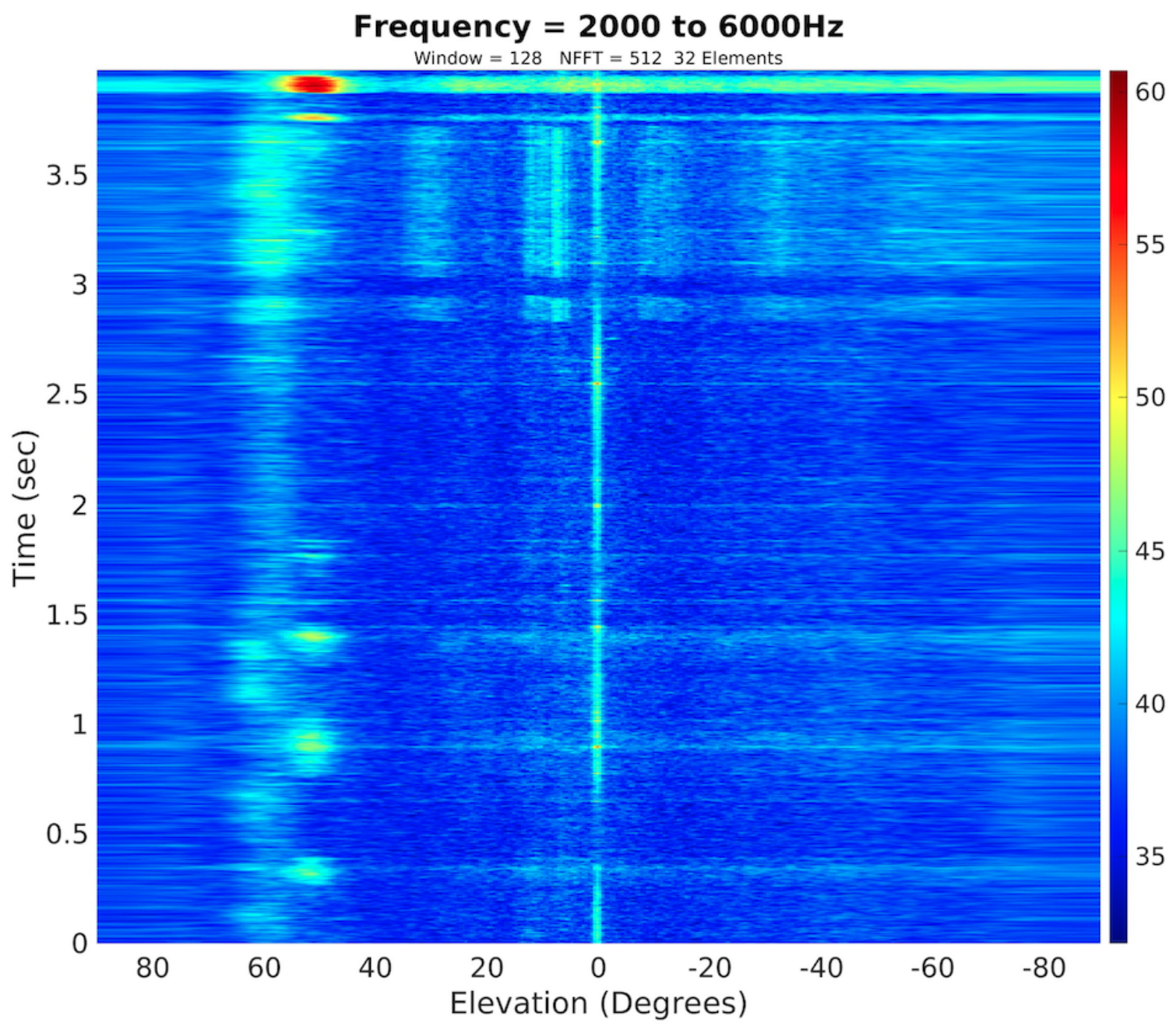

Figure B-11: DURIP Towed Array beamforming between $f=2000-6000 \mathrm{~Hz}$. The grating lobes are clearly present and resulting in spatial aliasing of the sound near $10^{\circ}$ between 2.8 to 3.6 seconds. However, the aliased signals are still fairly swathy and not sharply defined. 


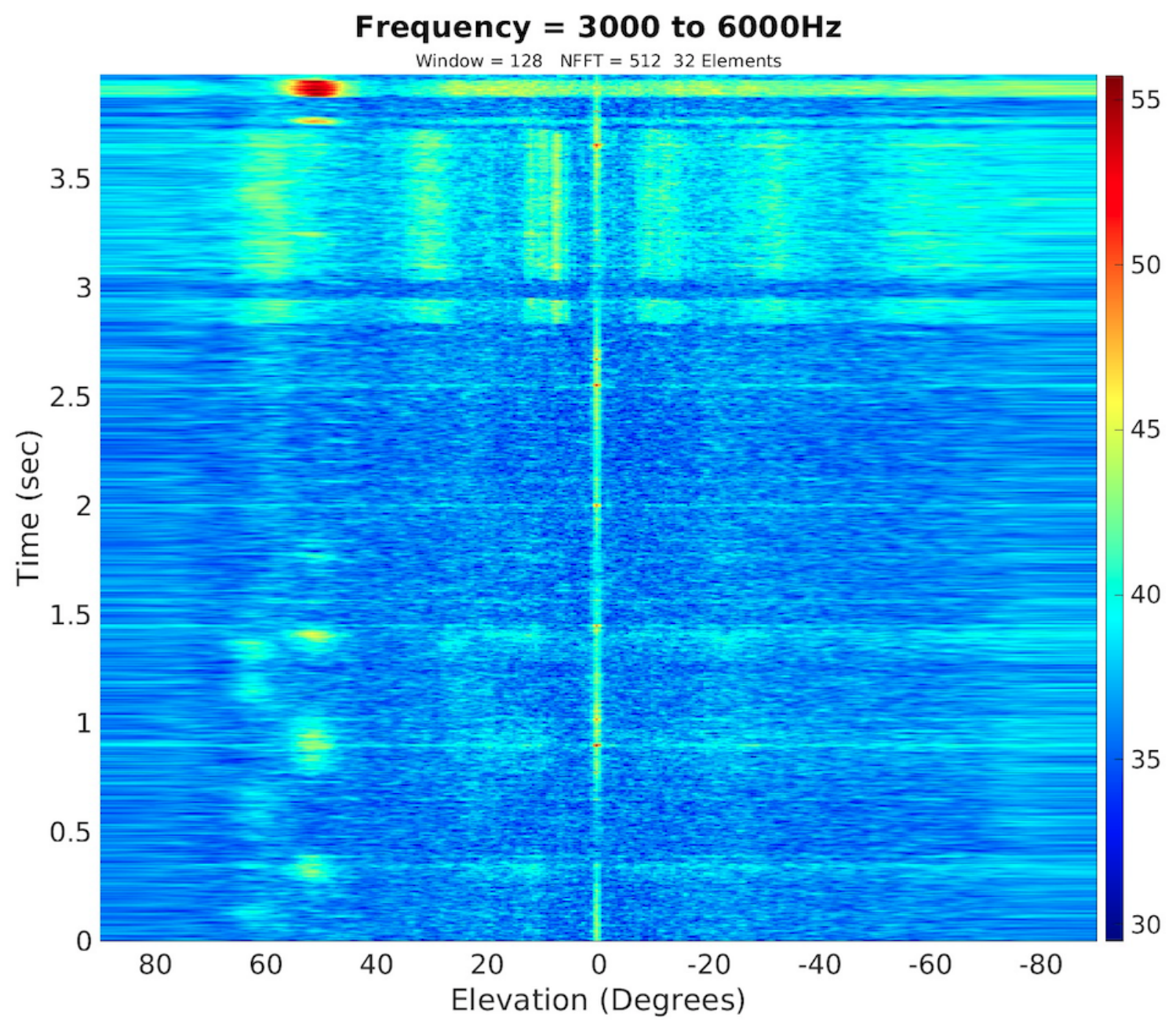

Figure B-12: DURIP Towed Array beamforming between $f=3000-6000 \mathrm{~Hz}$. The aliasing is clearly worse, but much of the low frequency content has been removed from the beamforming. Only higher frequency signals are present in this analysis. 


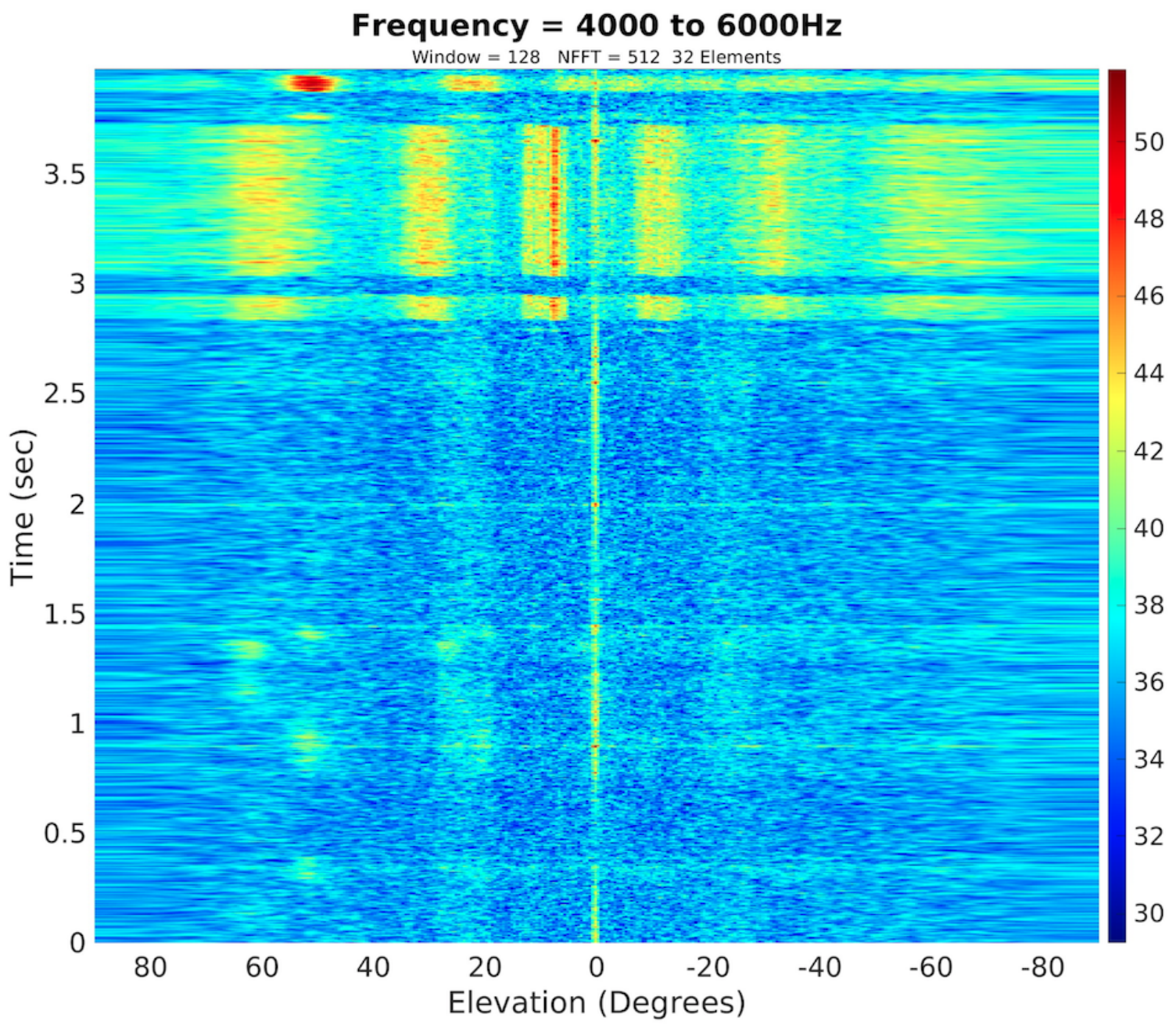

Figure B-13: DURIP Towed Array beamforming between $f=4000-6000 H z$. The spatial aliasing is bad. 


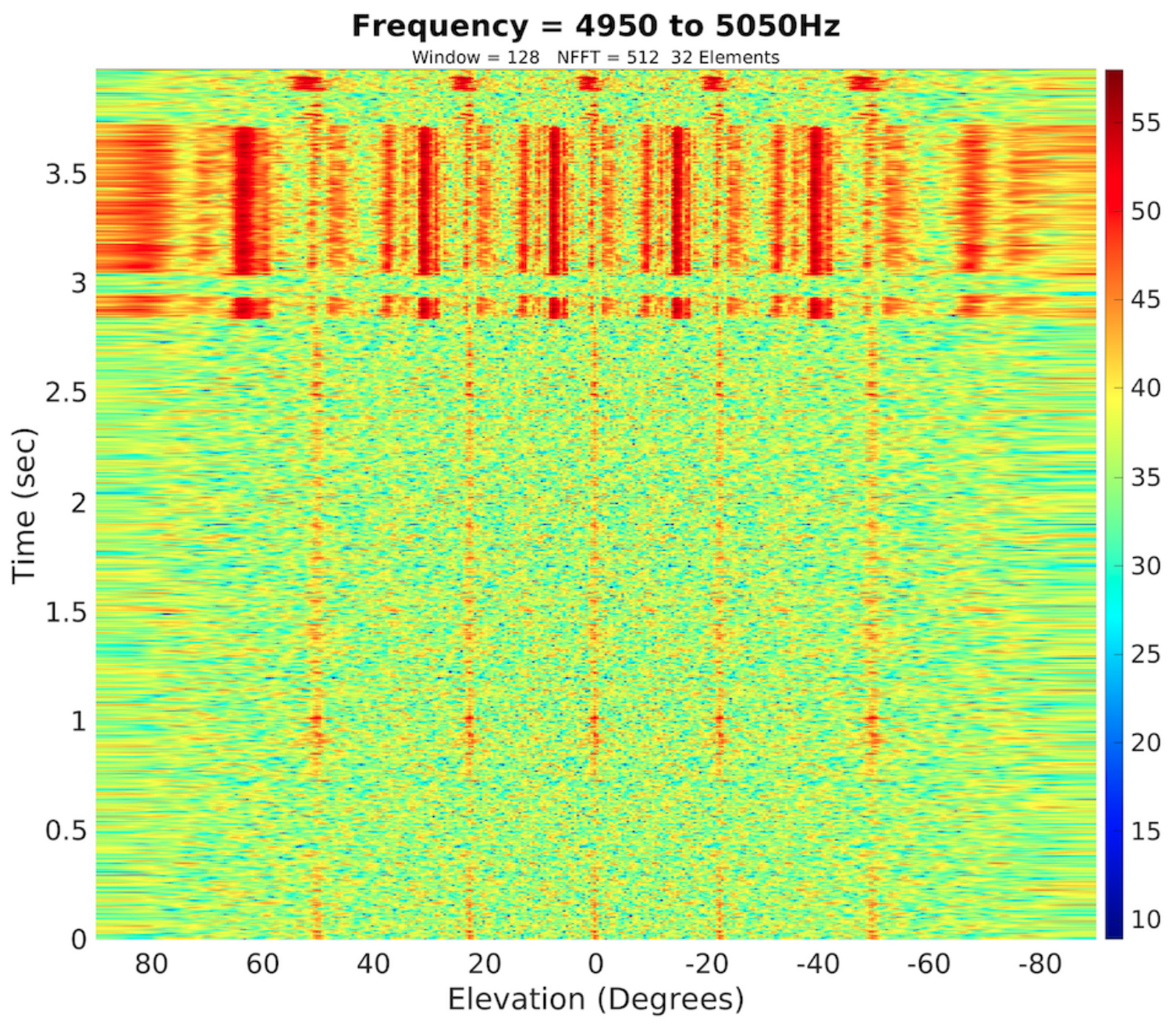

Figure B-14: DURIP Towed Array beamforming between $f=4950-5050 \mathrm{~Hz}$. The spatial aliasing in this case has an uncanny resemblance to the aliasing we would anticipate from Figure 4-1. This clearly shows how spatial aliasing occurs. The grating lobes effectively show sound at angles that it isn't actually present at. The transmission that occurs between 2.8 and 3.6 seconds is now spatially aliased, and it's unclear what angle the original signal was arriving from. The array noise that shows up at the beam is aliased to $\pm 50^{\circ}$ and $\pm 22^{\circ}$ which checks with the polar plot. 


\section{B.3.2 Macrura Transmission Grating Example}

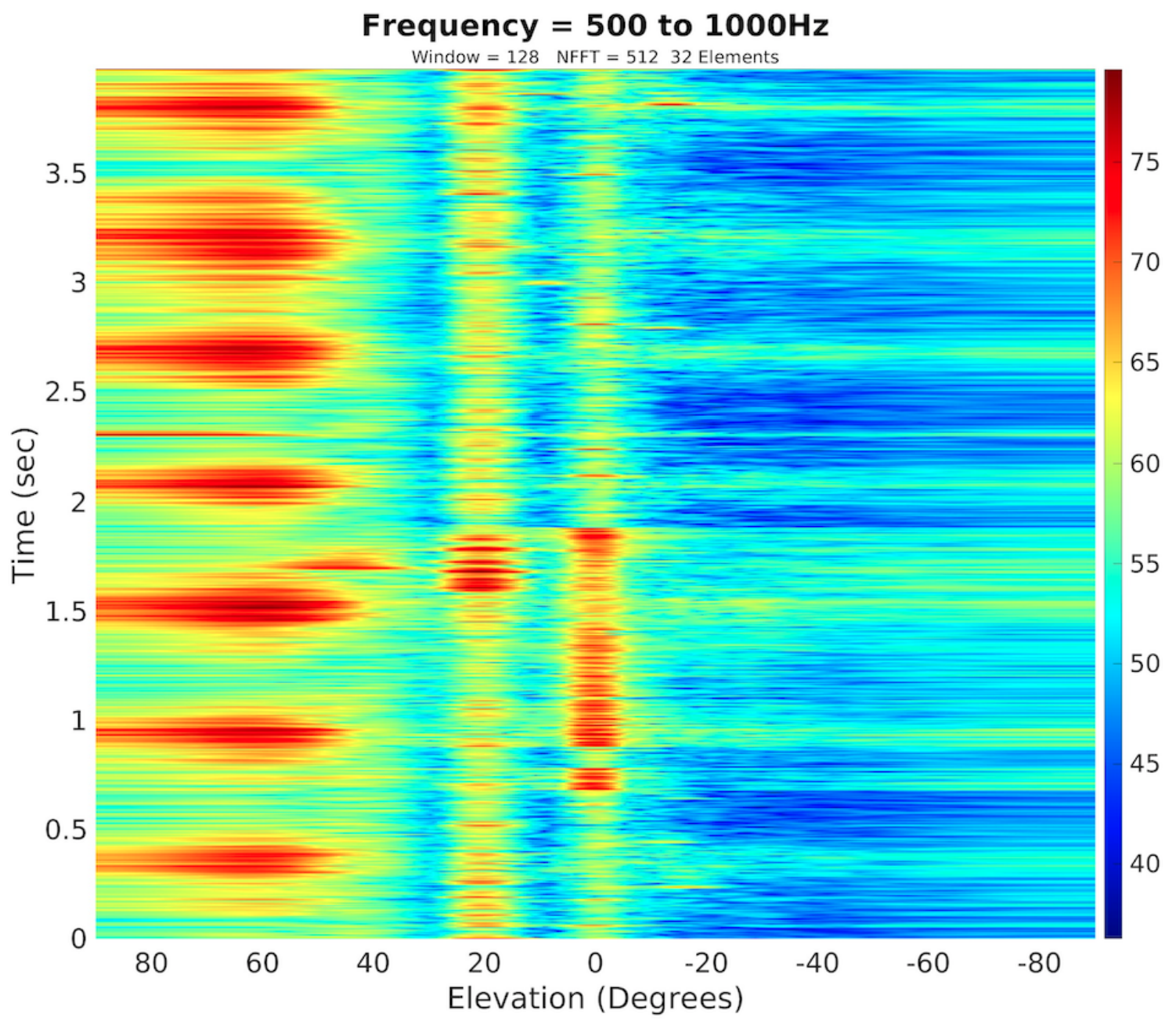

Figure B-15: DURIP Towed Array beamforming between $f=500-1000 \mathrm{~Hz}$, the designed spectrum for the array. The vehicle transmission should be in FWD endfire of the array but isn't visible in this spectrum. 


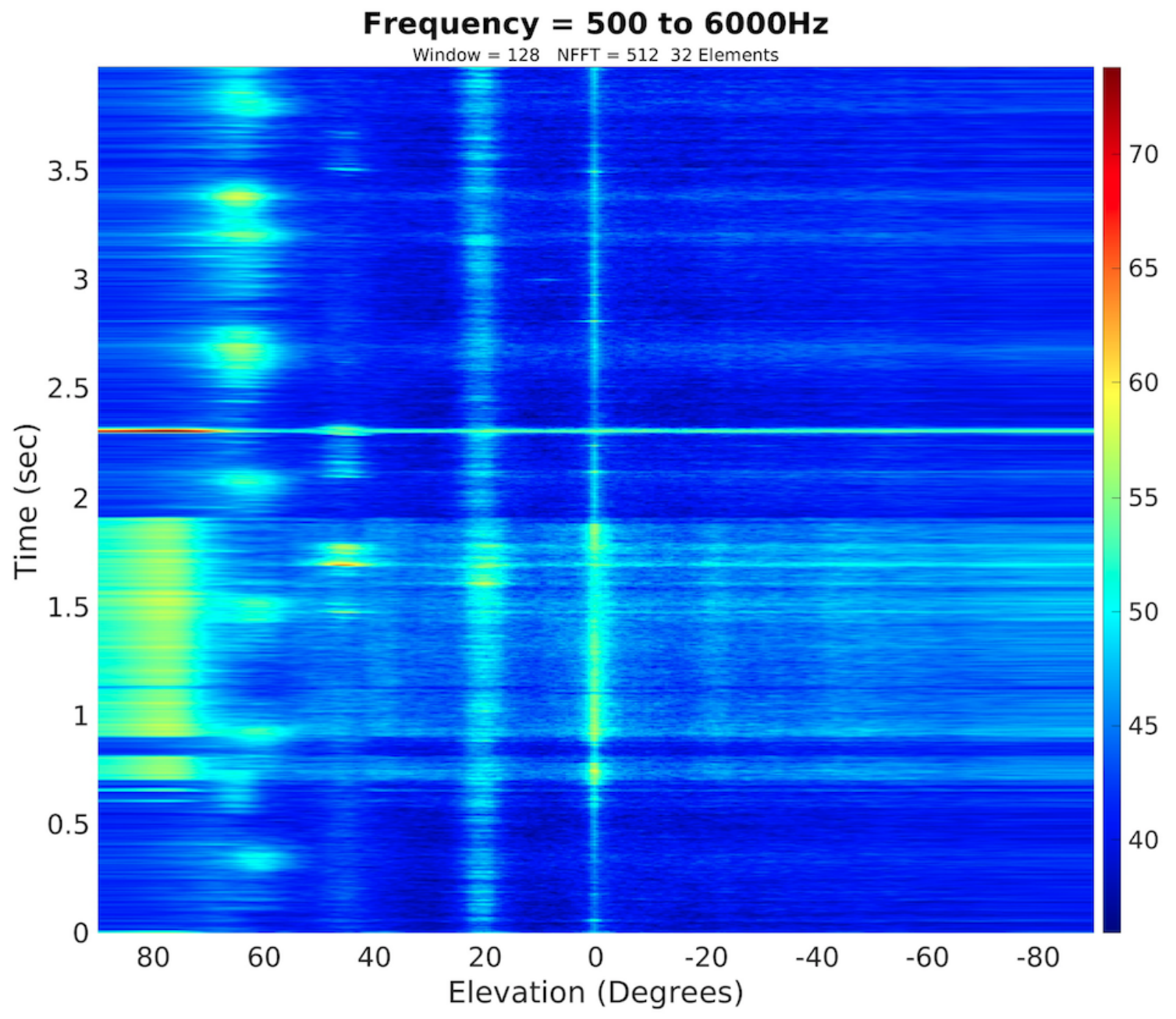

Figure B-16: DURIP Towed Array beamforming between $f=500-6000 \mathrm{~Hz}$. The vehicle transmission is visible in FWD endfire of the array. 


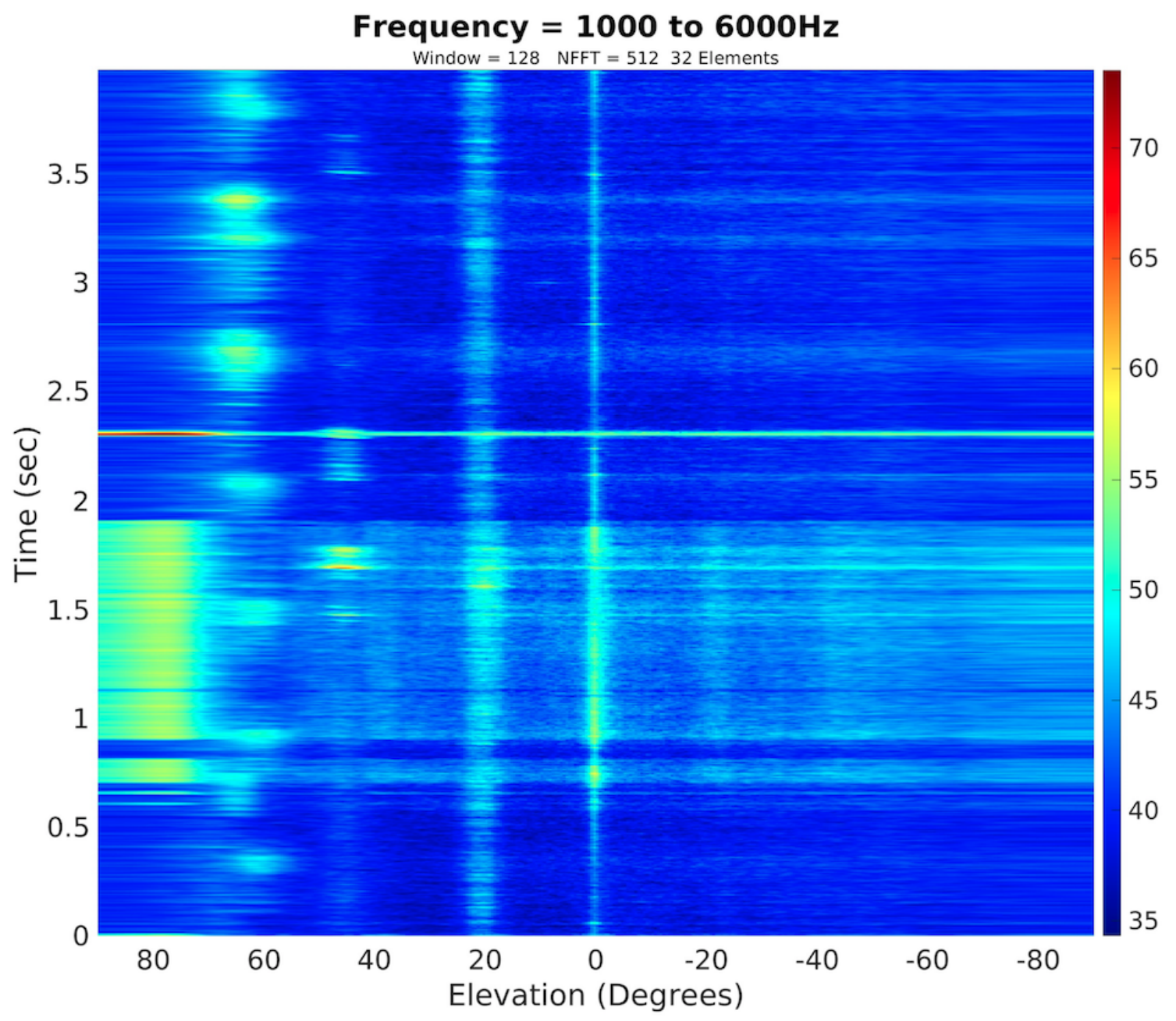

Figure B-17: DURIP Towed Array beamforming between $f=1000-6000 H z$. The vehicle transmission is visible in FWD endfire of the array. 


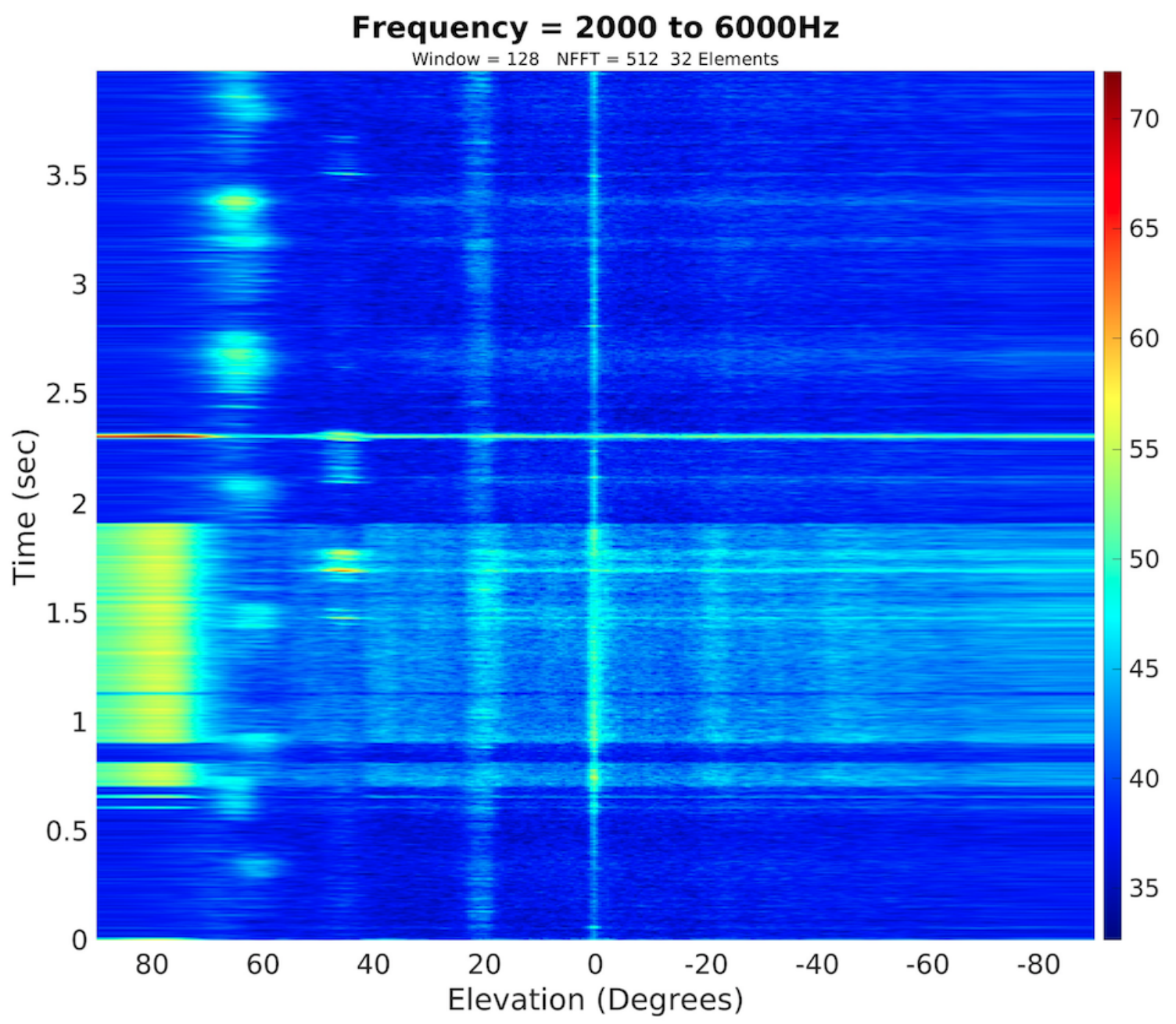

Figure B-18: DURIP Towed Array beamforming between $f=2000-6000 \mathrm{~Hz}$. The vehicle transmission is visible in FWD endfire of the array. Some minor aliasing is becoming apparent. 


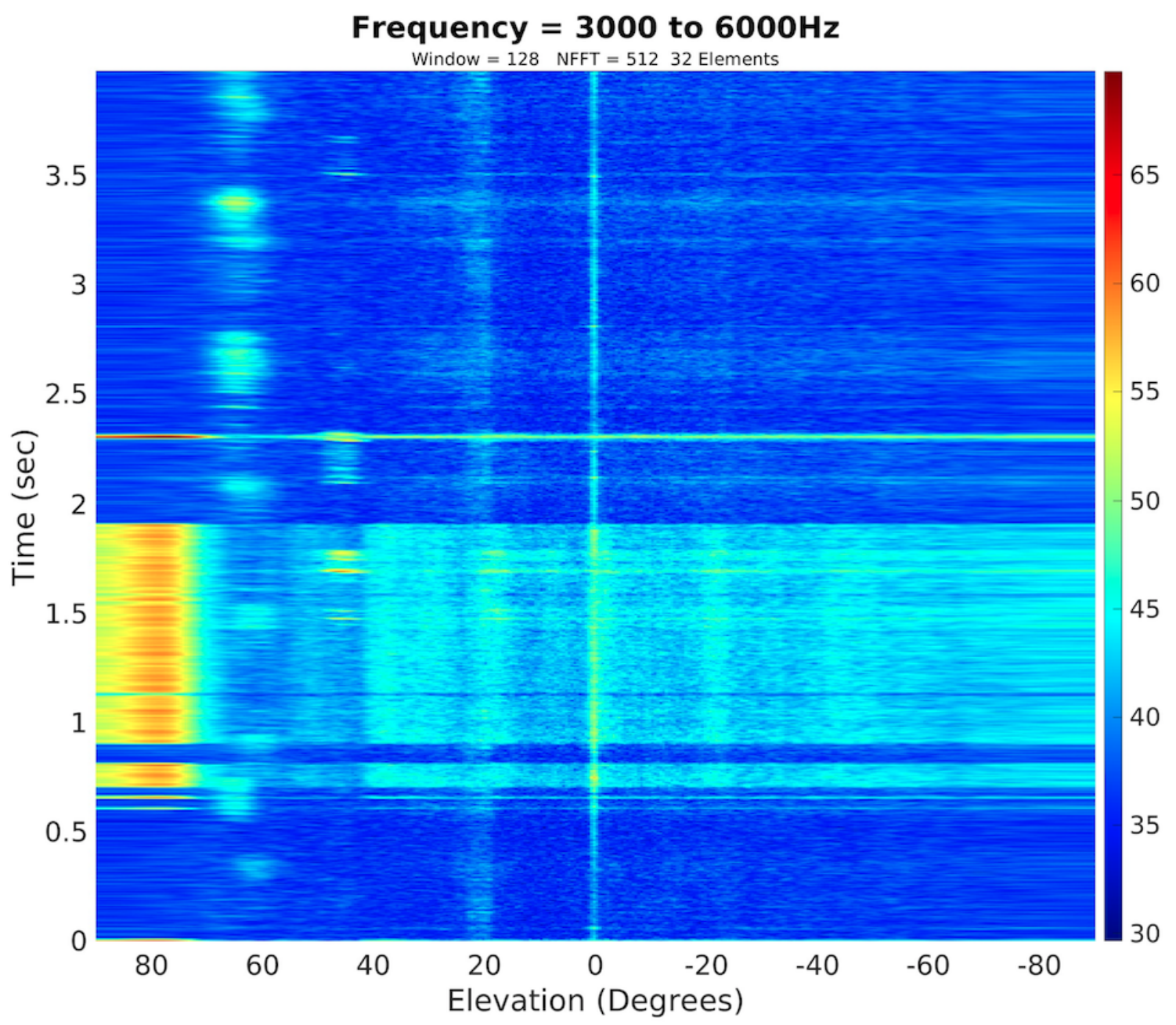

Figure B-19: DURIP Towed Array beamforming between $f=3000-6000 \mathrm{~Hz}$. The vehicle transmission is visible in FWD endfire of the array. Aliasing is causing almost all the beams to be washed out. FWD endfire remains the strongest, and actual source of the sound. 


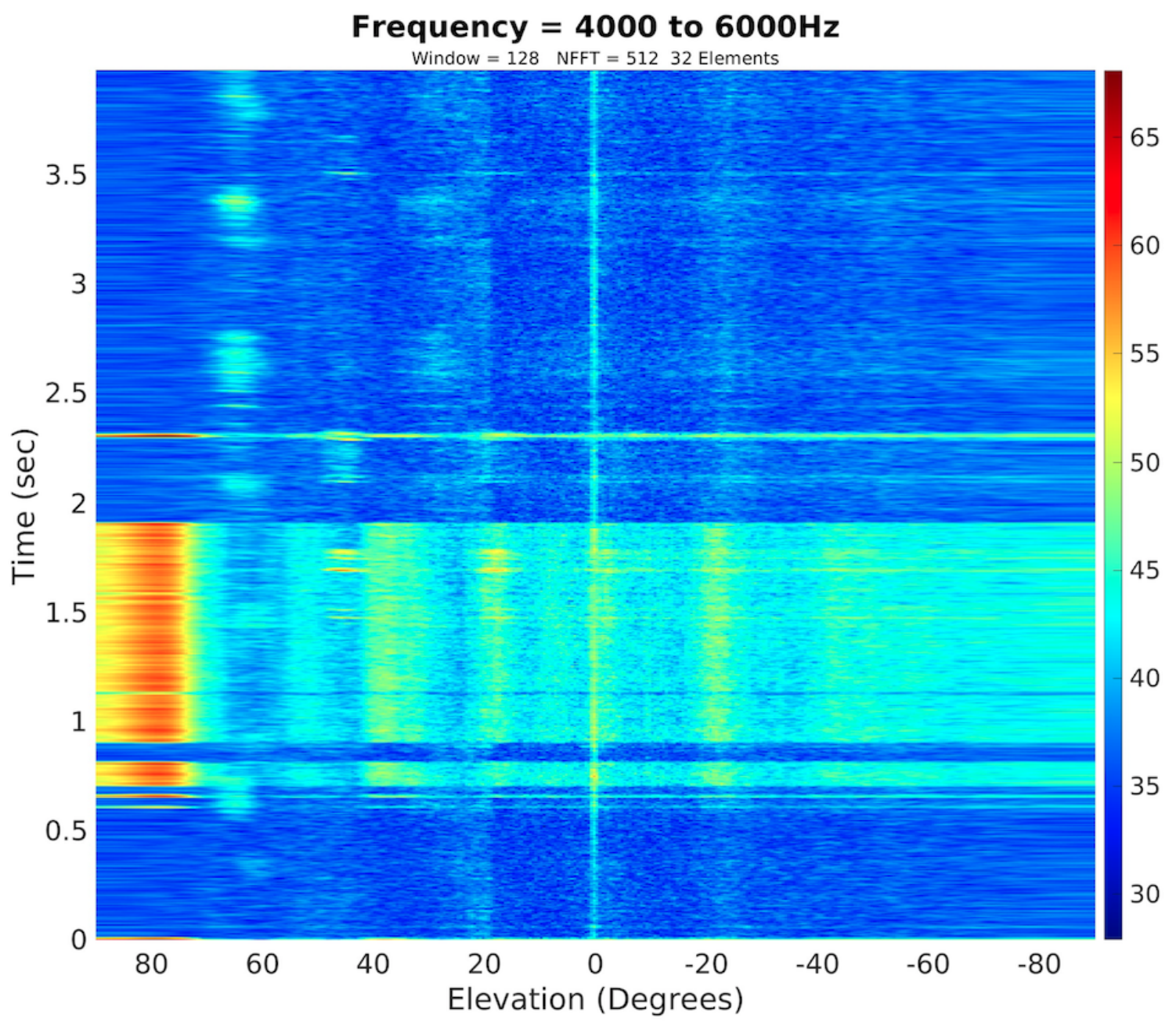

Figure B-20: DURIP Towed Array beamforming between $f=4000-6000 \mathrm{~Hz}$. The vehicle transmission is visible in FWD endfire of the array. Clear aliasing is happening. FWD endfire is still the most dominant angle for the transmission that is washing out the rest of the beams. 


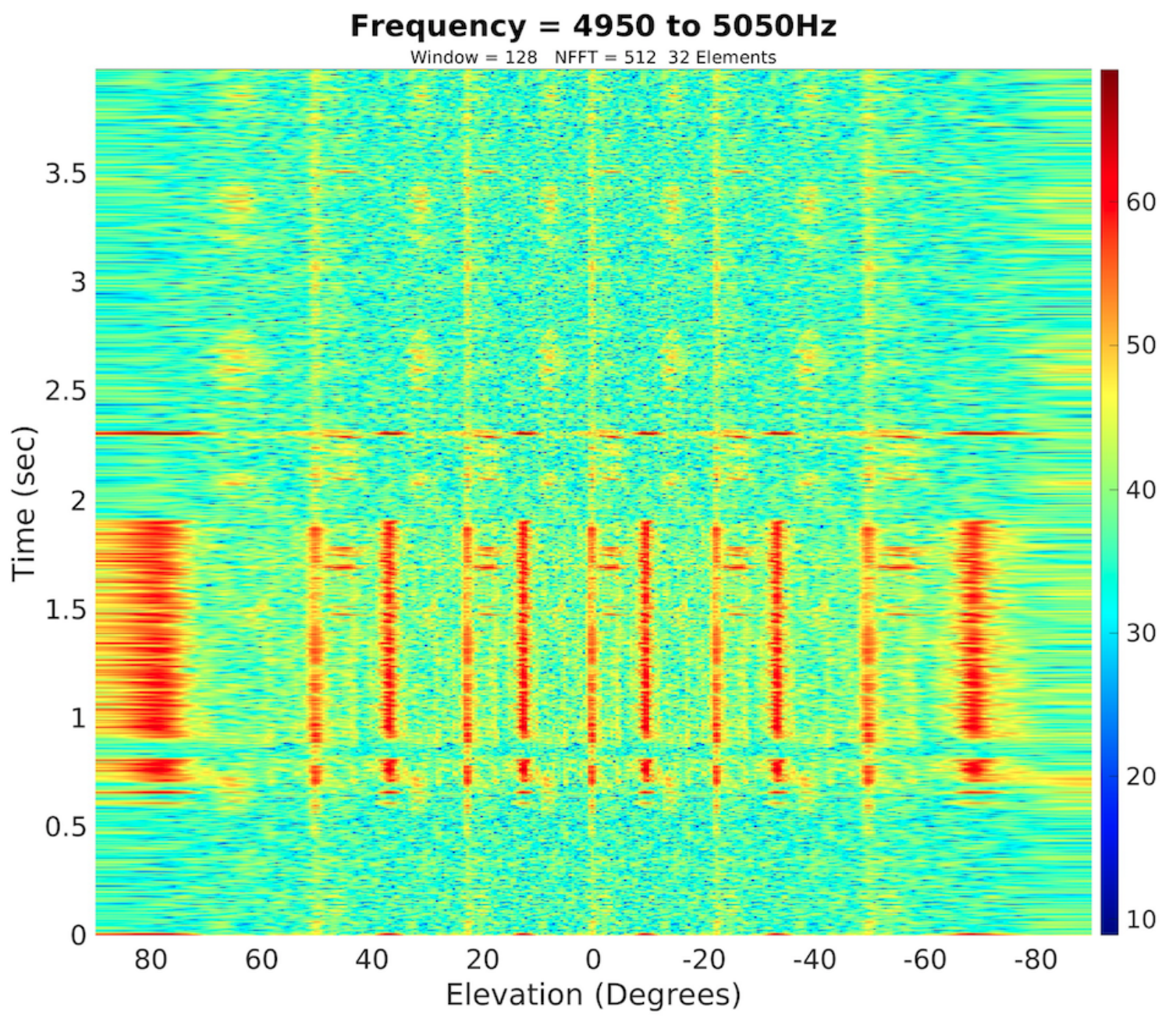

Figure B-21: DURIP Towed Array beamforming between $f=4950-5050 H z$. The vehicle transmission is visible in FWD endfire of the array. Aliasing is overwhelming here. The noise at $0^{\circ}$ is aliasing to $\pm 22^{\circ}$ and $\pm 50^{\circ}$. The original transmission angle is indeterminate now as aliasing dominates. Since the original transmission is present in the back lobe (Recall Figure 4-6b and 4-11) while the beam is steered to $90^{\circ}$ relative, the transmission is showing up subtly in the $0^{\circ} \mathrm{D} / \mathrm{E}$ as well by amplifying the already present signal there. 


\section{B.4 Vertical Run Additional Transmissions}

These are additional transmissions that were used to build the power spectral density (PSD),

Figure 1-13. These transmissions were recognizable and not washed out from the additional background noise present throughout the exercise.

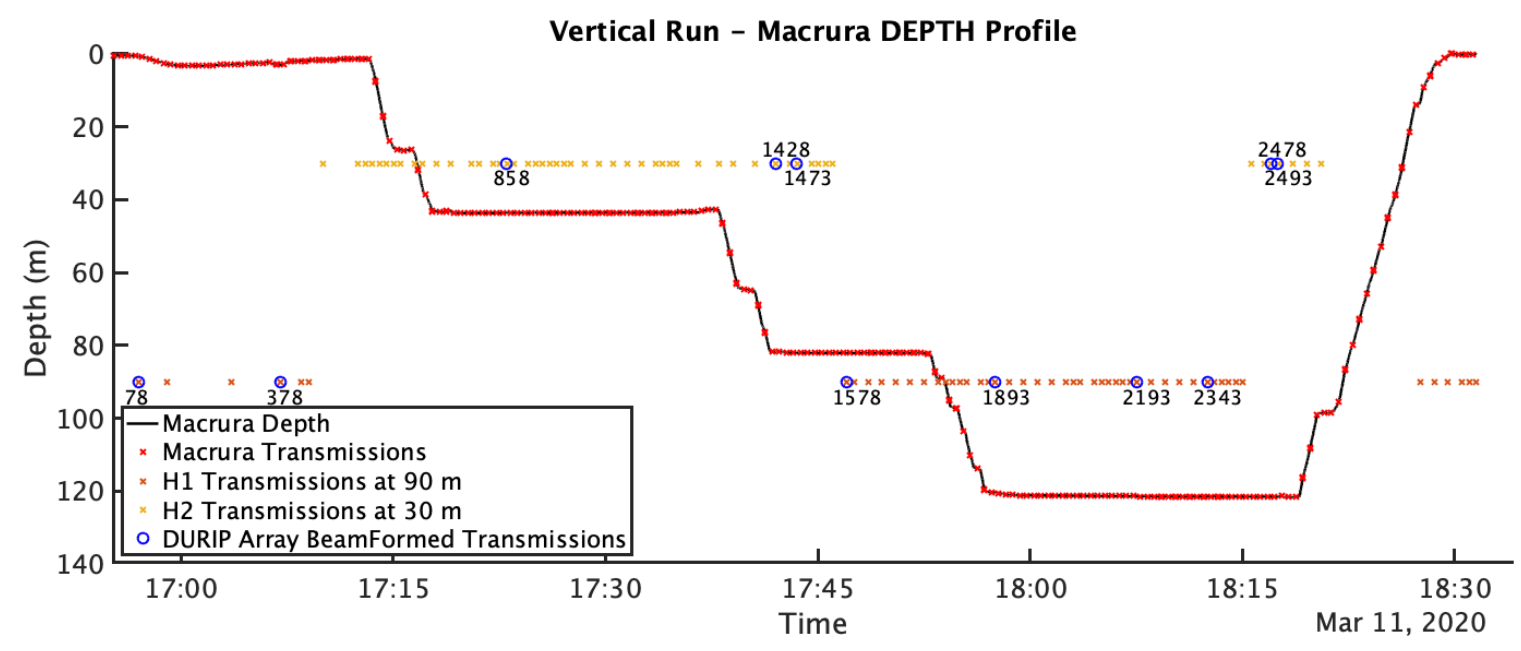

Figure B-22: Vertical Run highlighting the additional transmissions that were captured at each depth. 


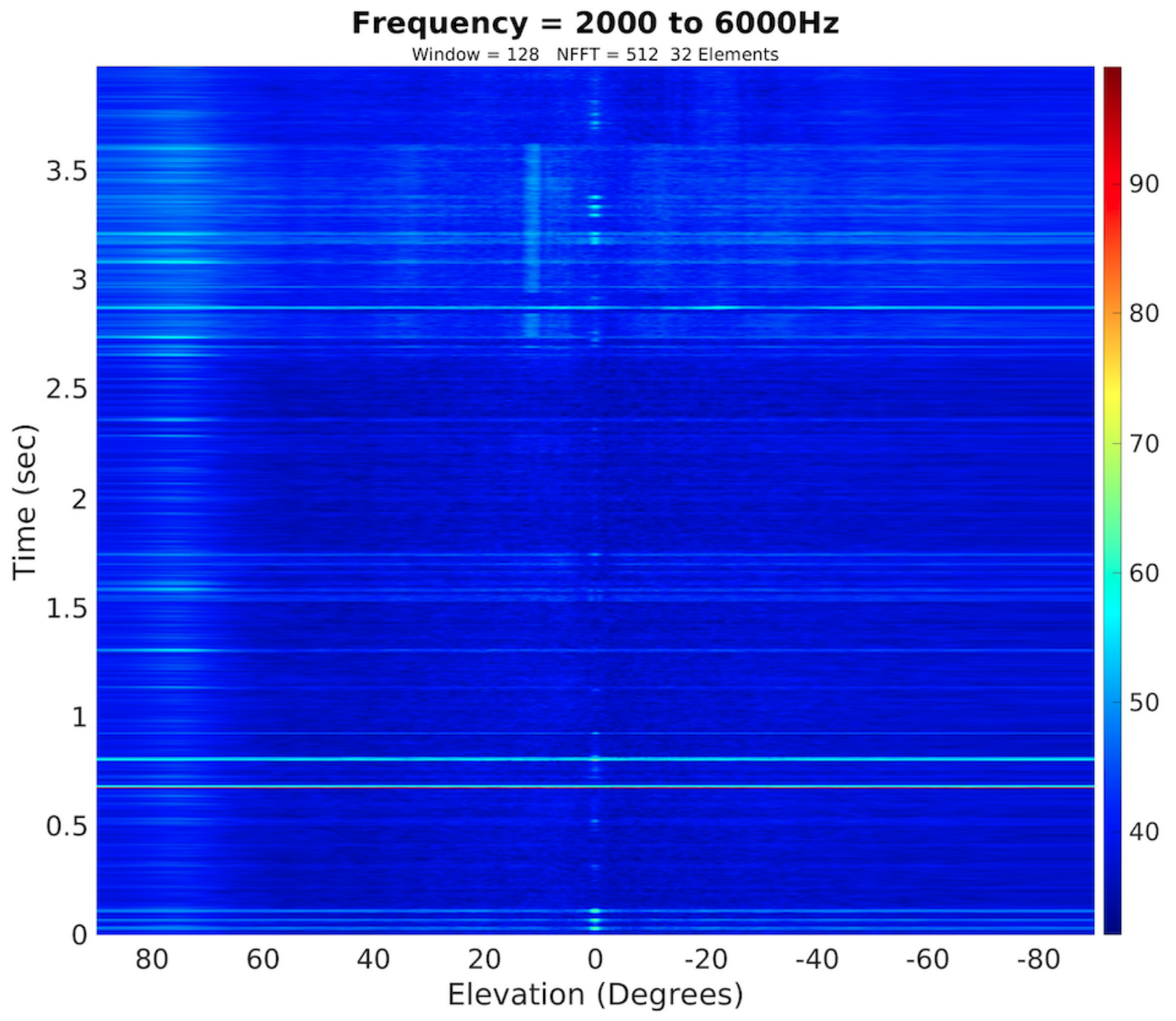

Figure B-23: Transmission \#78. $z_{s}=\mathrm{H} 190 \mathrm{~m}$. Array $\sim 38 \mathrm{~m}$. 


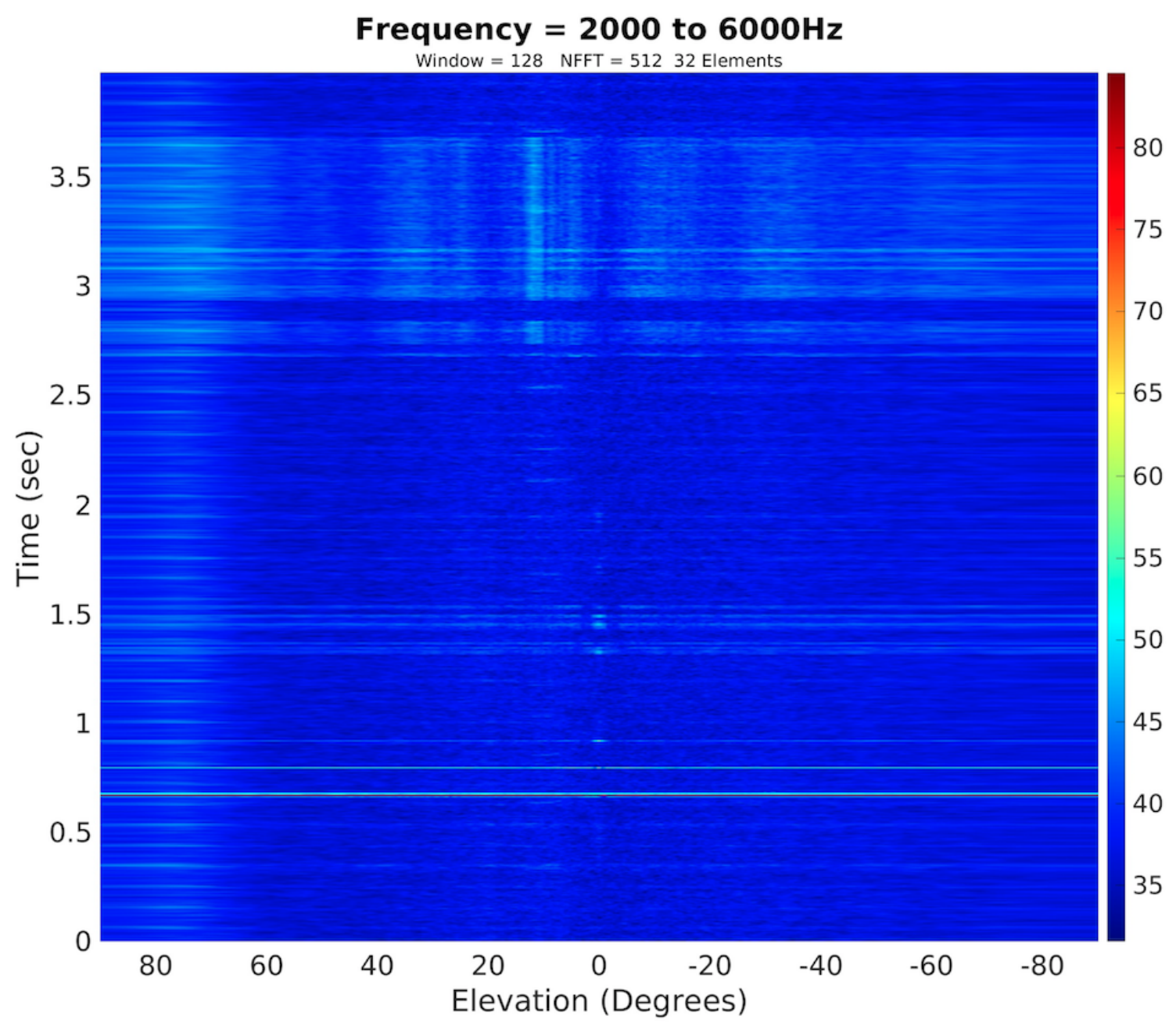

Figure B-24: Transmission \#378. $z_{s}=\mathrm{H} 190 \mathrm{~m}$. Array $\sim 38 \mathrm{~m}$. 


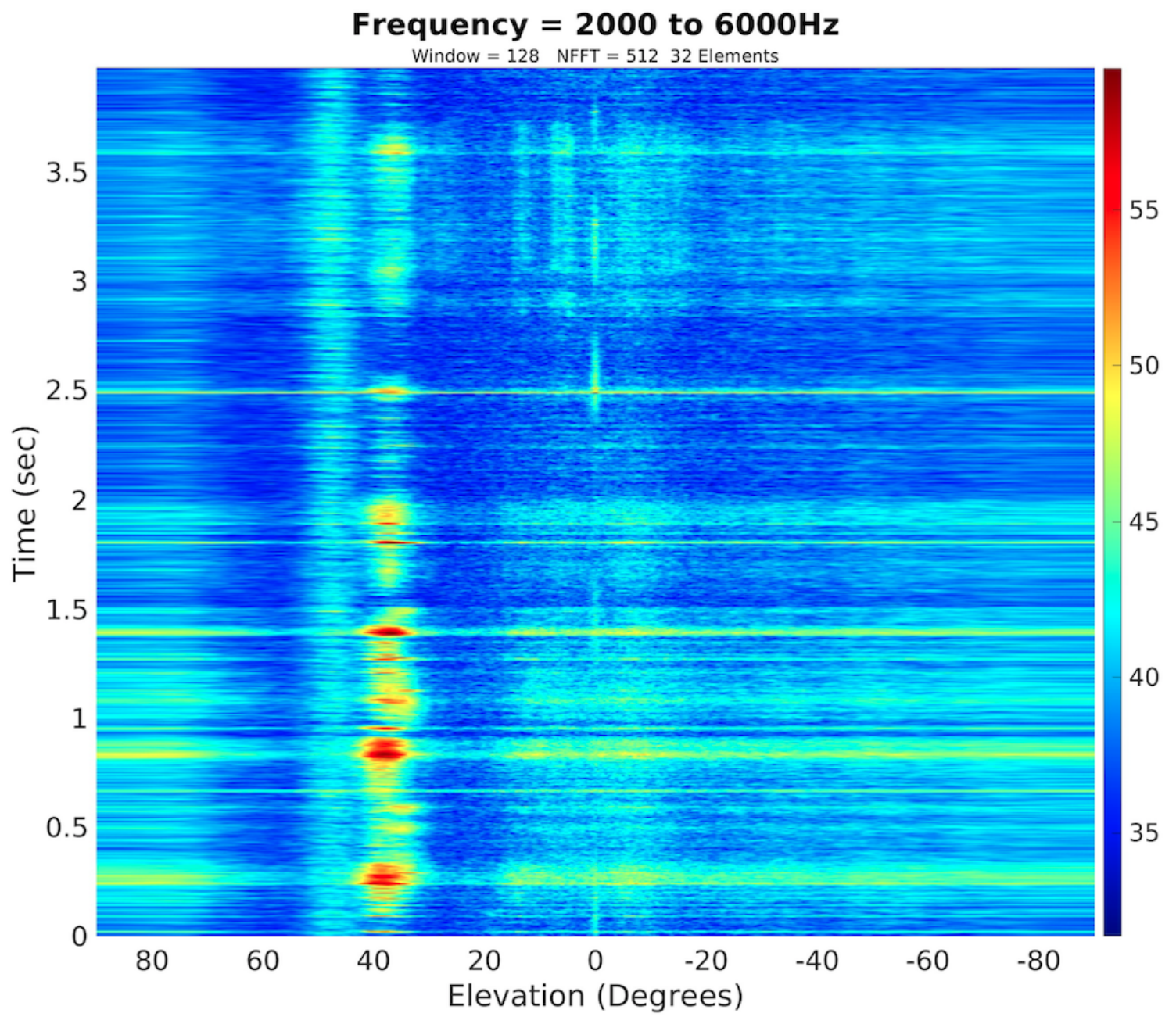

Figure B-25: Transmission \#858. $z_{s}=\mathrm{H} 230 \mathrm{~m}$. Array $\sim 82 \mathrm{~m}$. 


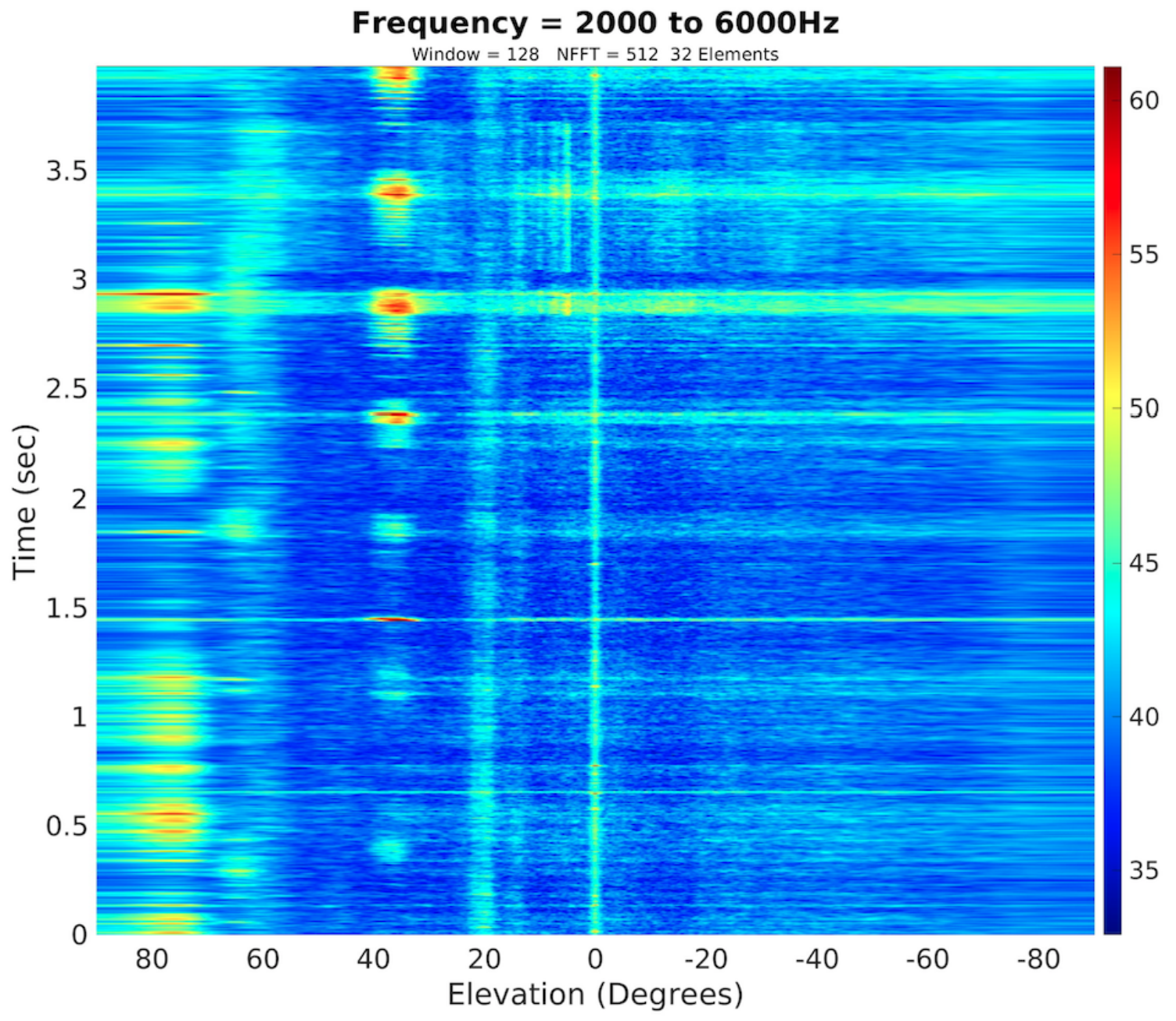

Figure B-26: Transmission \#1428. $z_{s}=\mathrm{H} 230 \mathrm{~m}$. Array $\sim 120 \mathrm{~m}$. 


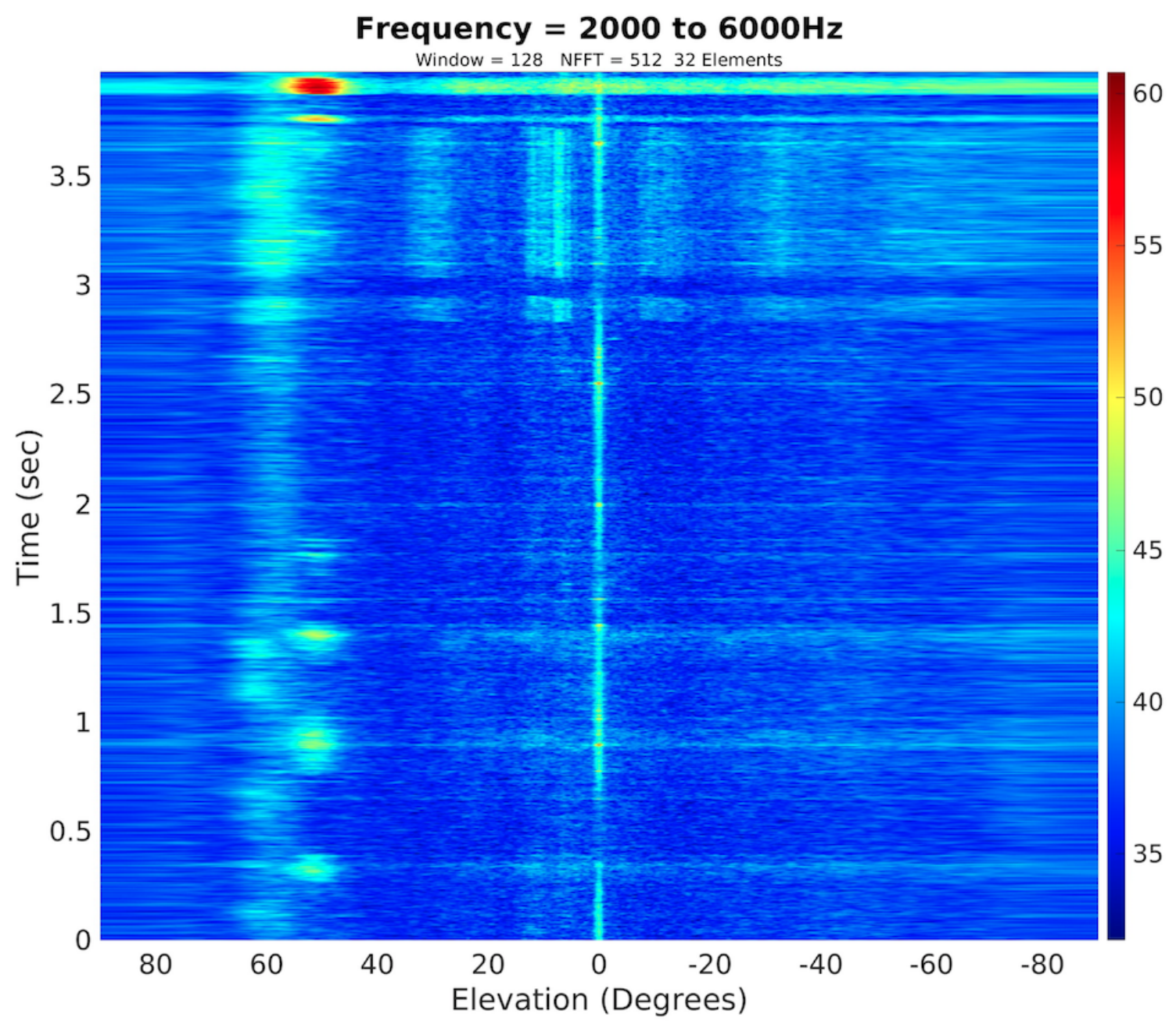

Figure B-27: Transmission \#1473. $z_{s}=\mathrm{H} 230 \mathrm{~m}$. Array $\sim 120 \mathrm{~m}$. 


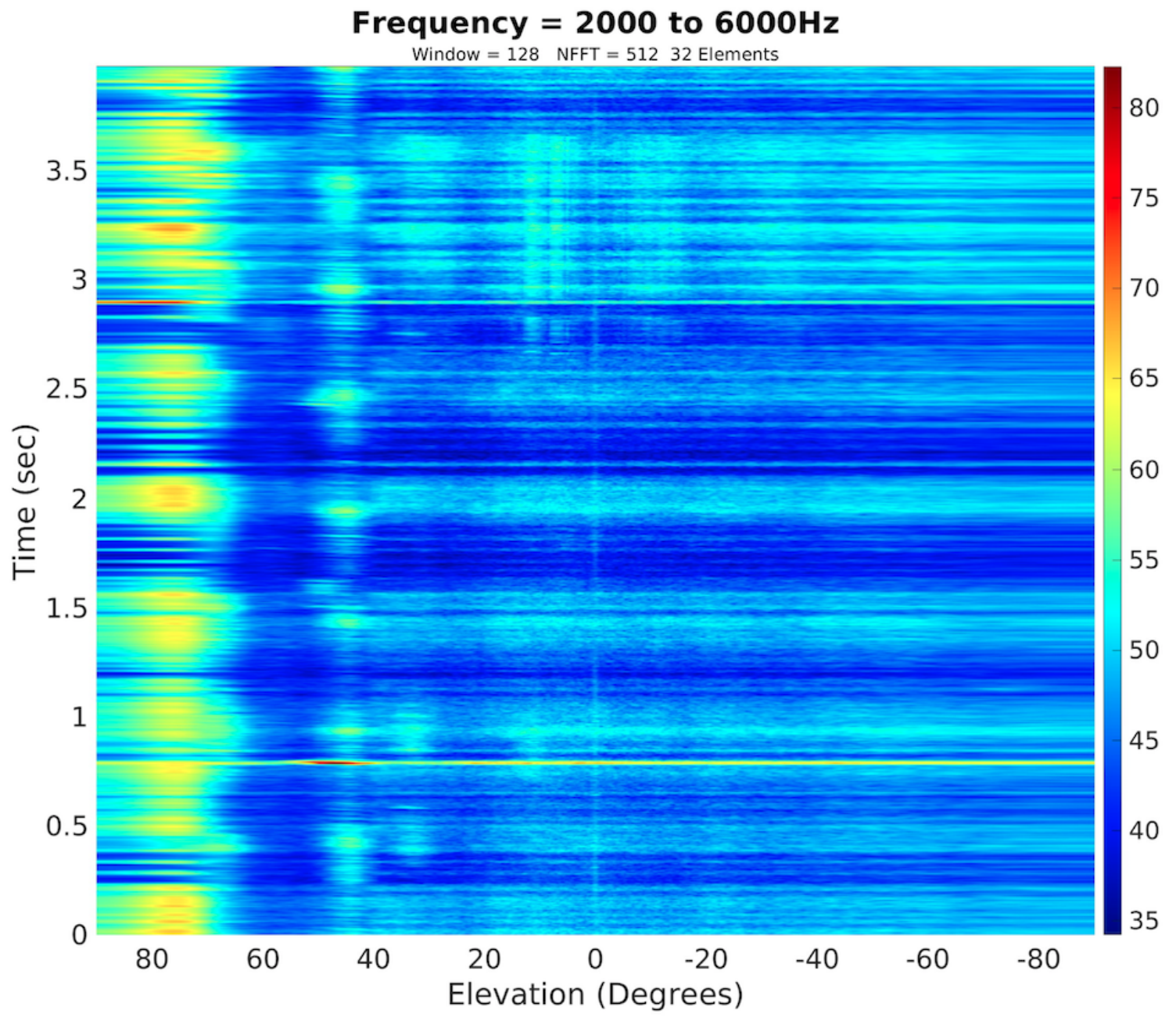

Figure B-28: Transmission \#1578. $z_{s}=\mathrm{H} 190 \mathrm{~m}$. Array $\sim 120 \mathrm{~m}$. 


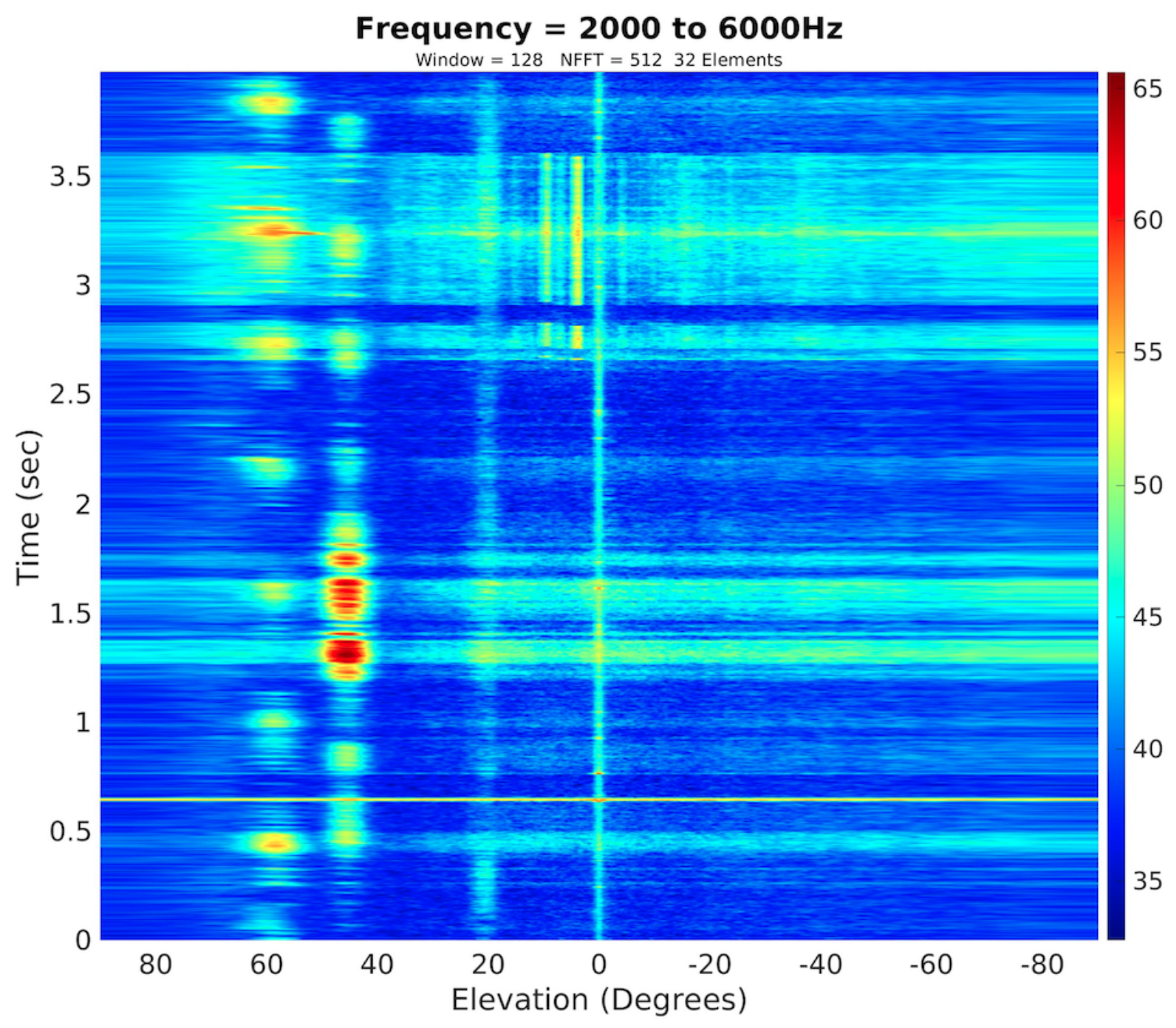

Figure B-29: Transmission \#1893. $z_{s}=$ H1 $90 \mathrm{~m}$. Array $\sim 159 \mathrm{~m}$. 


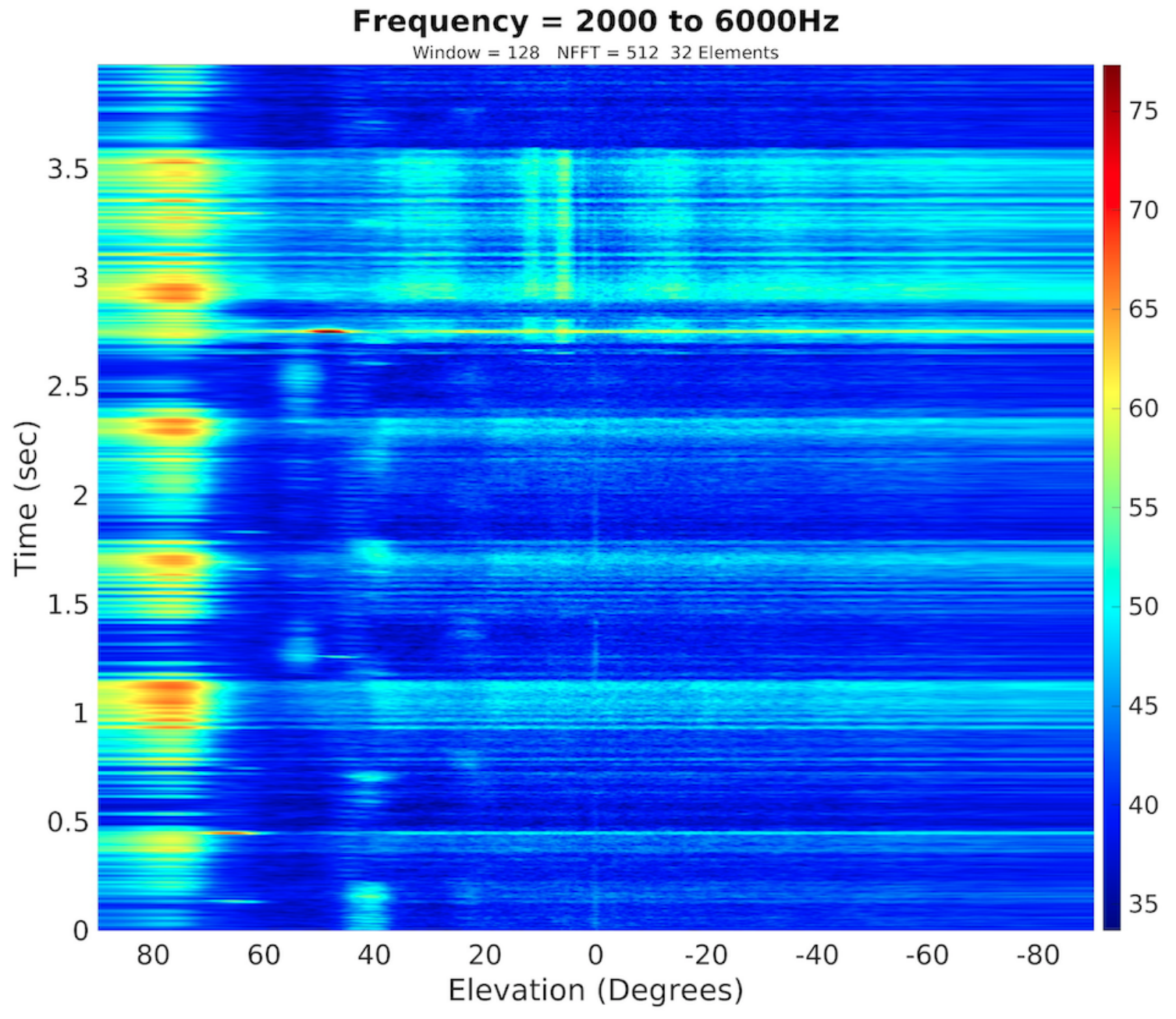

Figure B-30: Transmission \#2178. $z_{s}=\mathrm{H} 190 \mathrm{~m}$. Array $\sim 159 \mathrm{~m}$. 


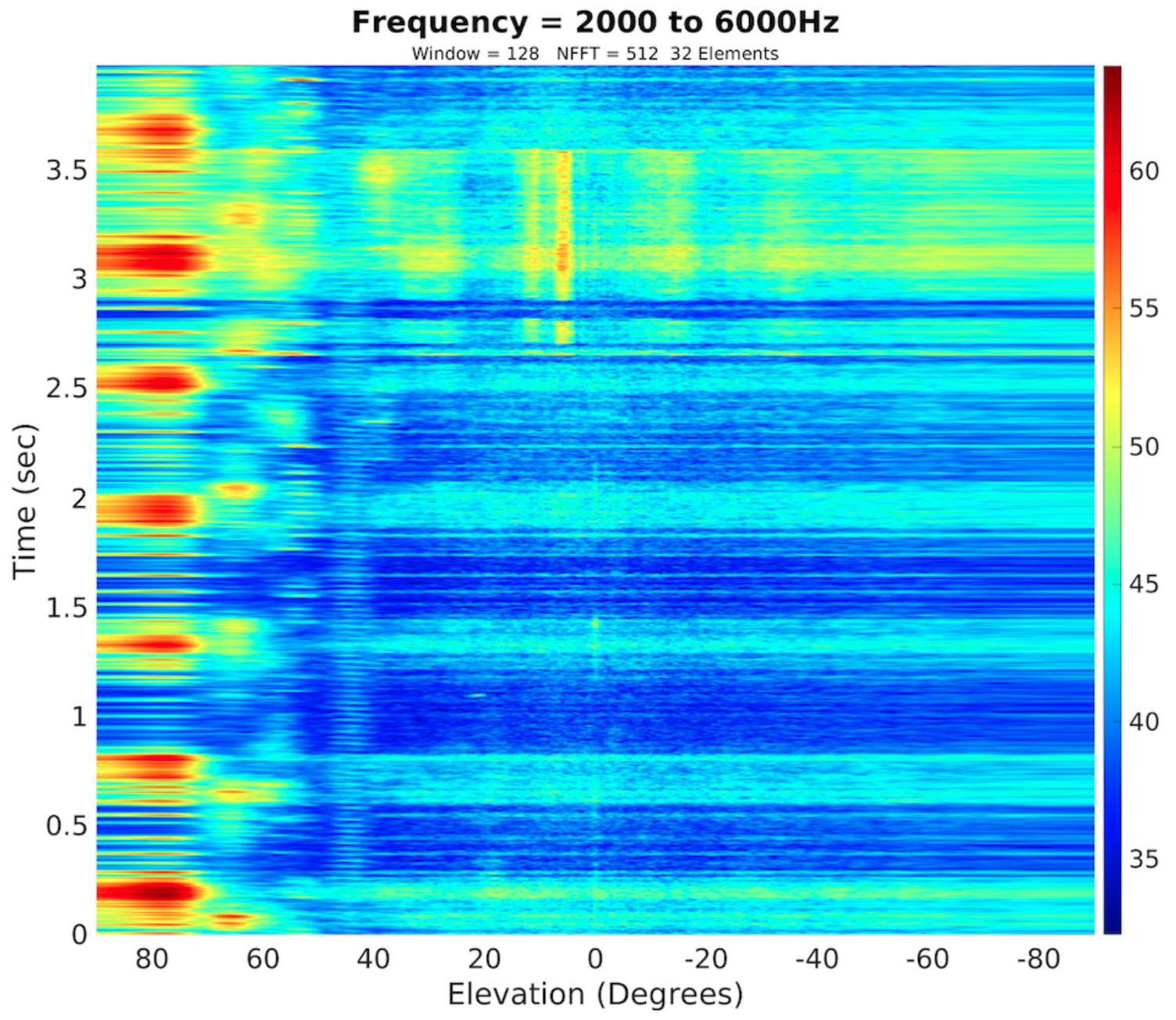

Figure B-31: Transmission \#2193. $z_{s}=\mathrm{H} 190 \mathrm{~m}$. Array $\sim 159 \mathrm{~m}$. 


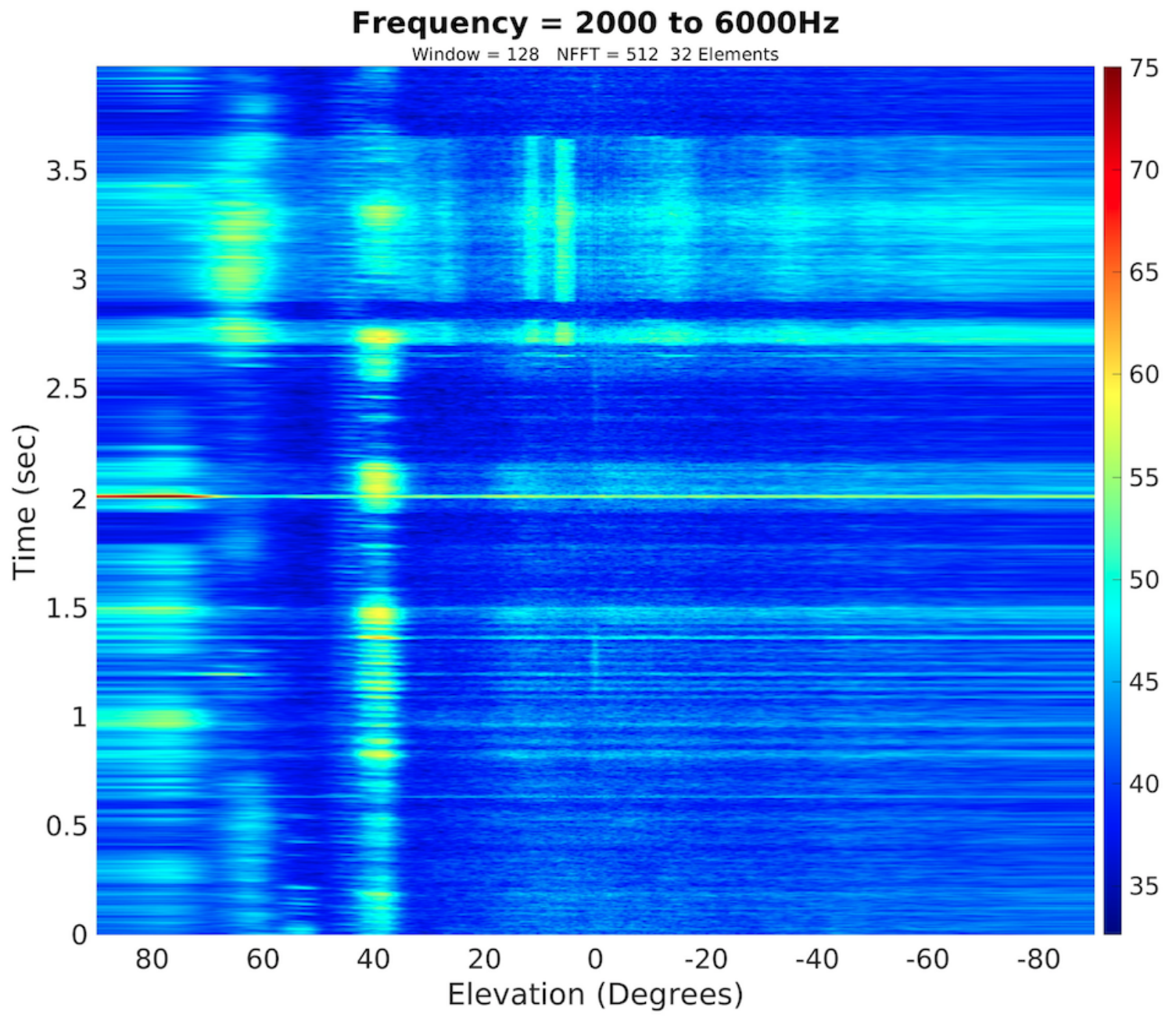

Figure B-32: Transmission \#2343. $z_{s}=\mathrm{H} 190 \mathrm{~m}$. Array $\sim 159 \mathrm{~m}$. 


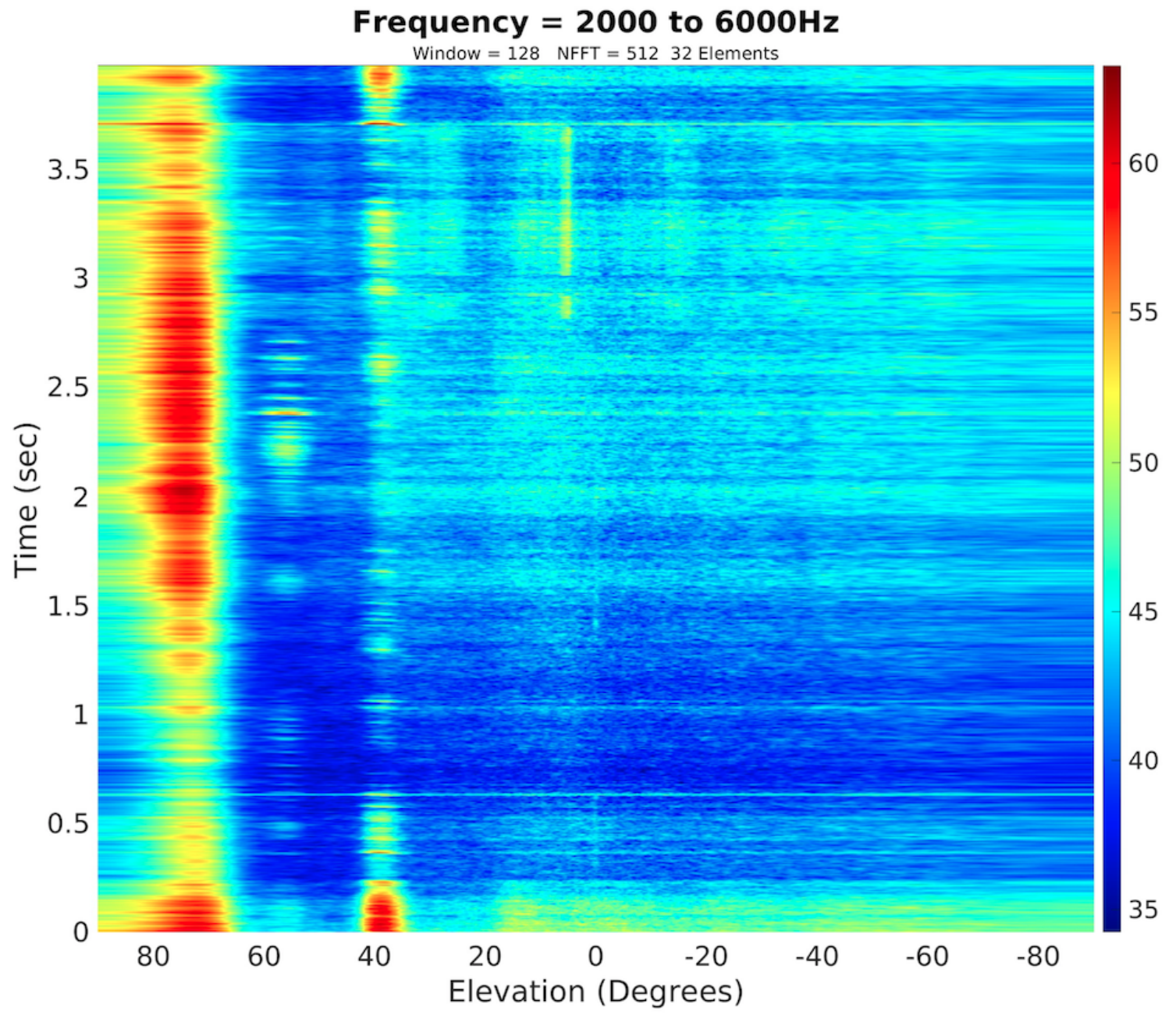

Figure B-33: Transmission \#2478. $z_{s}=\mathrm{H} 230 \mathrm{~m}$. Array $\sim 159 \mathrm{~m}$. 


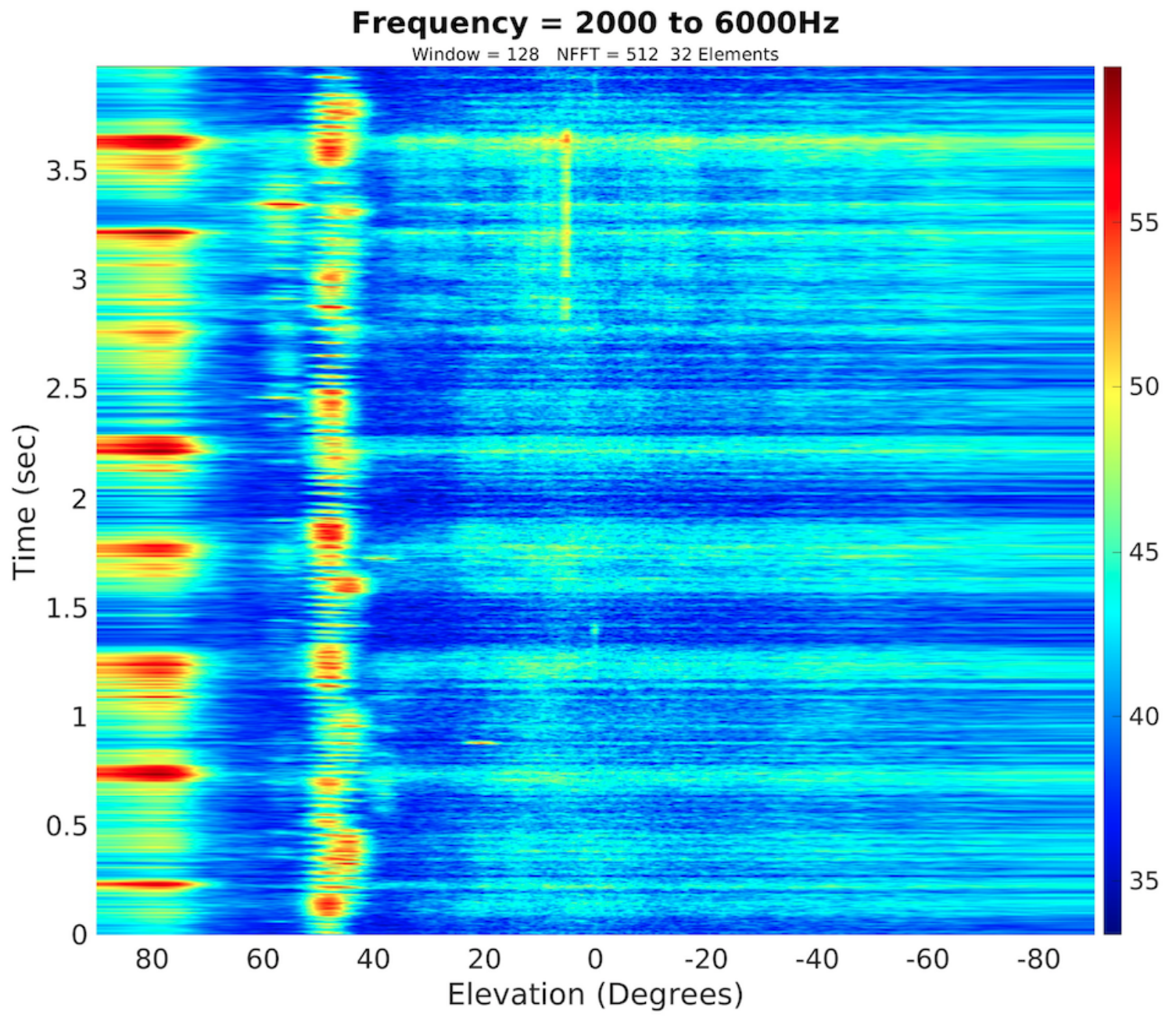

Figure B-34: Transmission \#2493. $z_{s}=\mathrm{H} 230 \mathrm{~m}$. Array $\sim 159 \mathrm{~m}$. 


\section{Bibliography}

[1] M. M. Gilday, K. J. Braithwaite II, and D. H. Berger, "A blue arctic," A Strategic Blueprint for the Arctic, 2021.

[2] H. Medwin and C. S. Clay, Fundamentals of acoustical oceanography. Academic press, 1997.

[3] F. B. Jensen, W. A. Kuperman, M. B. Porter, and H. Schmidt, Computational Ocean Acoustics. Springer Science \& Business Media, 2011.

[4] A. B. Baggeroer, H. Schmidt, S. A. Carper, J. Collis, and J. Rosenthal, "Detection and communication in the "beaufort lens"," The Journal of the Acoustical Society of America, vol. 140, no. 4, pp. 3408-3408, 2016. DOI: 10.1121/1.4970950. [Online]. Available: https://doi.org/10.1121/1.4970950.

[5] A. Poulsen and H. Schmidt, "Acoustic noise properties in the rapidly changing arctic ocean," vol. 28, Jan. 2016, p. 070005 . DOI: 10.1121/2.0000552.

[6] M. P. Langleben, "Attenuation of sound in sea ice, 10-500 khz," Journal of Glaciology, vol. 8, no. 54, pp. 399-406, 1969. DOI: 10.3189/S0022143000026988.

[7] S. Randeni, T. Schneider, and H. Schmidt, "Construction of a high-resolution underice auv navigation framework using a multidisciplinary virtual environment," in $A u$ tonomous Underwater Vehicles Symposium (AUV), IEEE, Oct. 2020.

[8] J. Preisig, "Acoustic propagation considerations for underwater acoustic communications network development," Mobile Computing Communications Review, vol. 11, no. 4, pp. 2-10, Oct. 2007.

[9] E. Jimenez, G. Quintana, P. Mena, P. Dorta, I. Perez-Alvarez, S. Zazo, M. Perez, and E. Quevedo, "Investigation on radio wave propagation in shallow seawater: Simulations and measurements," in 2016 IEEE Third Underwater Communications and Networking Conference (UComms), 2016, pp. 1-5. DOI: 10.1109/UComms.2016.7583453.

[10] A. Kenny and G. Lopez, "Advances in and extended application areas for doppler sonar," in 2012 Oceans, 2012, pp. 1-9. DOI: 10.1109/OCEANS.2012.6404884.

[11] T. R. Instruments, Workhorse navigator doppler velocity log (dvl), Accessed: 2021-0709, 2006. [Online]. Available: http://www.teledynemarine.com/Lists/Downloads/ navigator_datasheet_lr.pdf.

[12] A. Joseph, "Chapter 11 - vertical profiling of currents using acoustic doppler current profilers," eng, in Measuring Ocean Currents, Elsevier Inc, 2014, pp. 339-379, ISBN: 9780123914286.

[13] GeoSpectrum Technologies Inc., M27-930 ffr communications transducer, version 1, GTI, Dartmouth, Canada. 
[14] H. L. Van Trees, Optimum Array Processing. John Wiley \& Sons, Inc., 2002.

[15] PASCO, What type of wave is sound? https://www.pasco.com/resources/blog/237, Accessed: 2021-04-22, 2020.

[16] R. J. Urick and U. States., Sound propagation in the sea / written for Defense Advanced Research Projects Agency [by] R. J. Urick, English. DARPA ; for sale by the Supt. of Docs., U.S. Govt. Print. Off [Arlington, Va.] : Washington, D.C, 1979, ca. 300 p. in various pagings :

[17] R. E. Francois and G. R. Garrison, "Sound absorption based on ocean measurements. part ii: Boric acid contribution and equation for total absorption," The Journal of the Acoustical Society of America, vol. 72, no. 6, pp. 1879-1890, 1982. DOI: 10.1121/1. 388673. [Online]. Available: https://doi.org/10.1121/1.388673.

[18] National Snow \& Ice Data Center, Salinity and brine, Accessed: 2021-06-22, 2020. [Online]. Available: https ://nsidc .org/cryosphere/seaice/characteristics / brine_salinity.html.

[19] Naval Oceanographic Office, Fleet oceanographic and acoustic reference manual, United States Navy, Stennis Space Center, MS, Apr. 1999.

[20] M. B. Porter, The bellhop manual and user's guide: Preliminary draft, https://oalibacoustics.org/AcousticsToolbox/Bellhop-2010-1.pdf, 2011.

[21] J. H. McClellan, R. W. Schafer, and M. A. Yoder, Signal Processing First. Pearson Education, Inc., 2003.

[22] M. Lt. Pelisssero. (Mar. 2020). "U.s. navy kicks off icex 2020 in arctic ocean," [Online]. Available: https : / www .norad.mil/Newsroom/Article/2104840/us - navy-kicks off-icex-2020-in-arctic-ocean/.

[23] S. Snow. (Mar. 2020). "Intercepted russian reconnaissance aircraft were watching us submarines during icex," [Online]. Available: https : / / www . militarytimes . com / flashpoints / 2020 / 03 / 11 / intercepted - russian - reconnaissance - aircraft were-watching-us-submarines-during-icex/.

[24] H. Schmidt, J. Leonard, and D. Battle, "Goats 2005 integrated, adaptive autonomous acoustic sensing systems," Sep. 2006.

[25] T. Schneider and H. Schmidt, "Model-based adaptive behavior framework for optimal acoustic communication and sensing by marine robots," IEEE Journal of Oceanic Engineering, vol. 38, pp. 522-533, 2013.

[26] H. Schmidt and A. Balasuriya, "Goats 2011 adaptive and collaborative exploitation of 3-dimensional environmental acoustics in distributed undersea networks," Sep. 2013.

[27] E. C. Bhatt, "A virtual ocean framework for environmentally adaptive, embedded acoustic navigation on autonomous underwater vehicles," Ph.D. dissertation, Massachusetts Institute of Technology and Woods Hole Oceanographic Institute, Boston, MA, Sep. 2021. DOI: 10.1575/1912/27309.

[28] R. B. Blackman and J. W. Tukey, The measurement of power spectra, from the point of view of communications engineering, eng. New York: Dover Publications, 1958.

[29] D. P. Bertsekas and J. N. Tsitsiklis, Introduction to Probability, Second Edition, eng. Massachusetts: Athena Scientific, 2008, ISBN: 978-1-886529-23-6. 
[30] H. Consortium. (). "Hycom overview," [Online]. Available: https://www.hycom.org/ hycom/overview.

[31] Acoustic Communications Group, Micromodem-2 user's guide, version 1.5, Woods Hole Oceanographic Institution, Falmouth, Massachusetts, Aug. 2017. 